\title{
Copper-Catalyzed Tandem Cross-Coupling/Thermally-Promoted [2 + 2] Cycloaddition of 1,6-Enynes and Diazo Compounds to Assemble Methylenecyclobutane-Fused Ring System
}

Nuan Chen,${ }^{\dagger}$ Ting Zhou, ${ }^{\dagger}$ Hong Zhang, ${ }^{\dagger}$ Yuqi Zhu,${ }^{\dagger}$ Ming Lang, ${ }^{\dagger}$ Jian Wang, $,{ }^{\dagger}, \dot{\dagger}$ and Shiyong Peng $*, \dagger$

'School of Biotechnology and Health Sciences, Wuyi University, Jiangmen 529020, P. R. China

${ }^{+}$School of Pharmaceutical Sciences, Key Laboratory of Bioorganic Phosphorous Chemistry \& Chemical Biology (Ministry of Education), Tsinghua University, Beijing, 100084, P. R. China E-mails: psy880124@mail.nankai.edu.cn;wangjian2012@tsinghua.edu.cn

\section{Table of Contents}

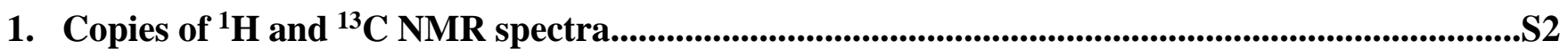

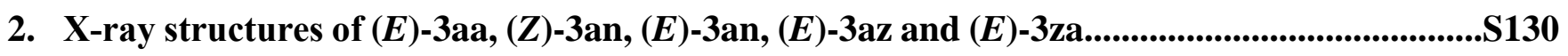




\section{Copies of ${ }^{1} \mathrm{H}$ and ${ }^{13} \mathrm{C}$ NMR spectra}

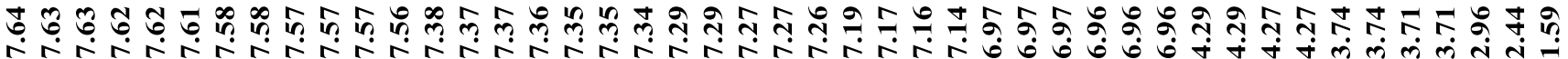

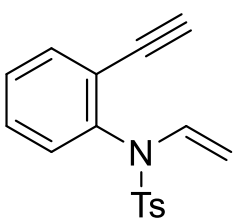

1a

$\left(500 \mathrm{MHz}\right.$; in $\left.\mathrm{CDCl}_{3}\right)$

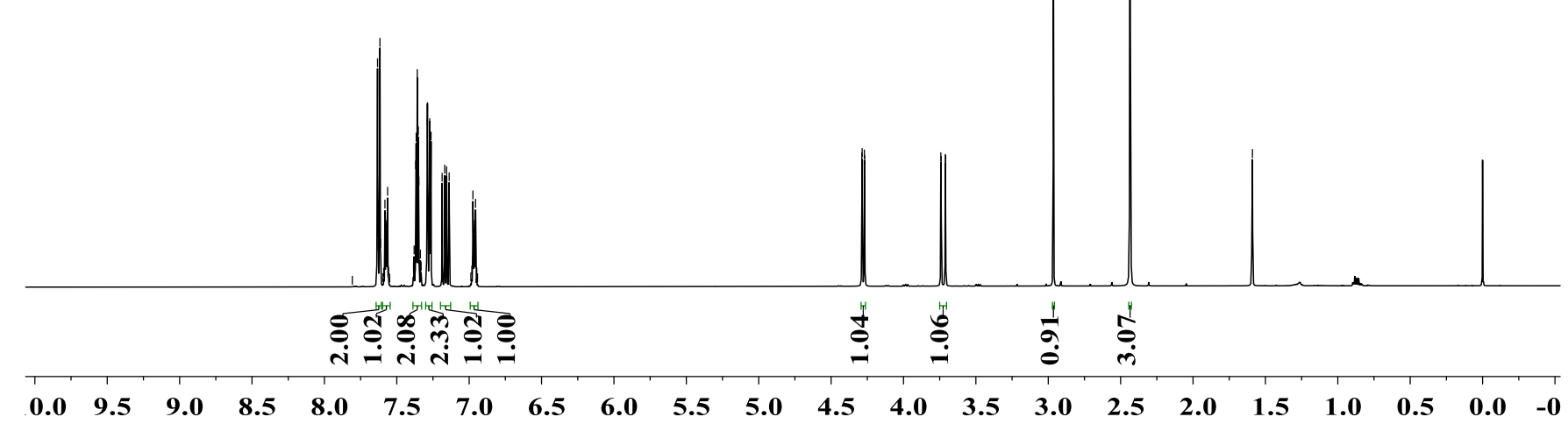

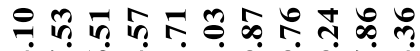

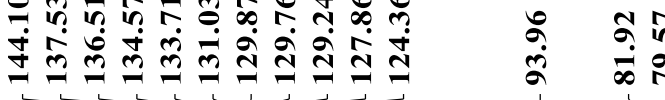

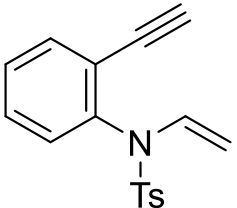

$1 \mathrm{a}$

$\left(125 \mathrm{MHz}\right.$; in $\left.\mathrm{CDCl}_{3}\right)$

$\begin{array}{llllllllllllllllllllllllllll}200 & 190 & 180 & 170 & 160 & 150 & 140 & 130 & 120 & 110 & 100 & 90 & 80 & 70 & 60 & 50 & 40 & 30 & 20 & 10 & 0 & -10\end{array}$ 

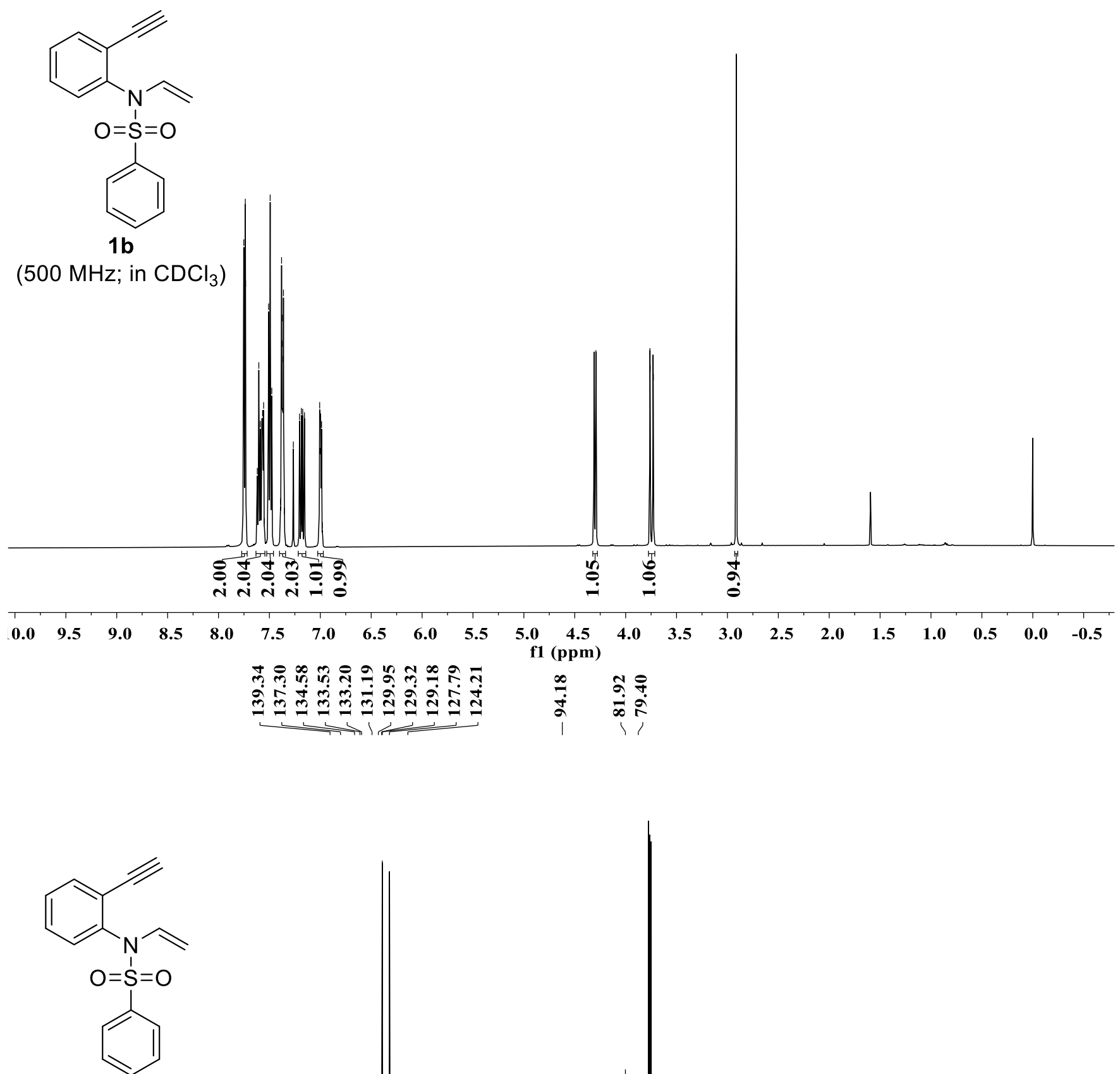

$1 \mathrm{~b}$

(125 MHz; in $\mathrm{CDCl}_{3}$ ) 


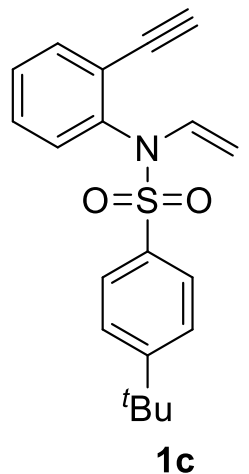

$\left(500 \mathrm{MHz}\right.$; in $\left.\mathrm{CDCl}_{3}\right)$
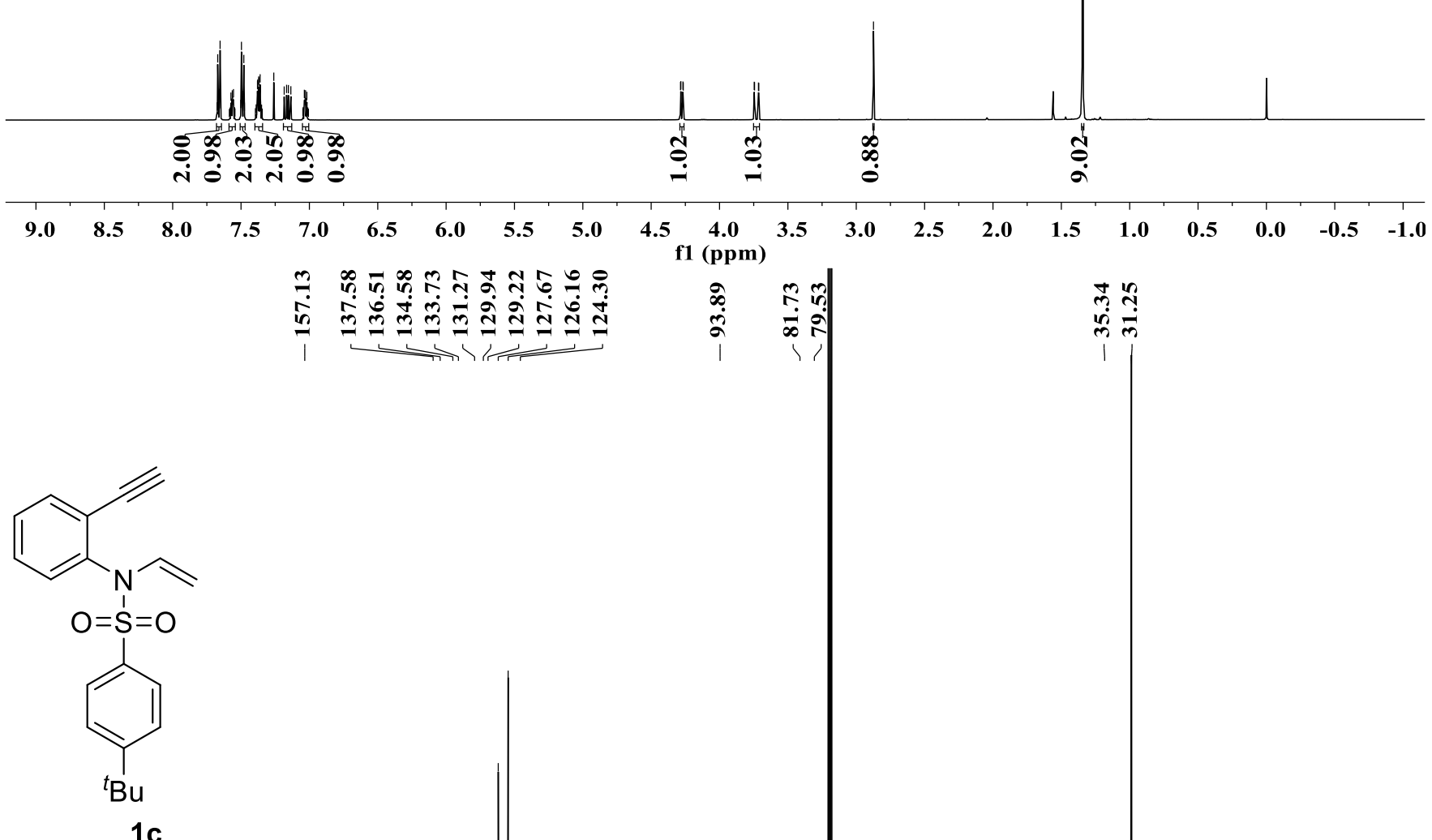

(125 MHz; in $\mathrm{CDCl}_{3}$ ) 


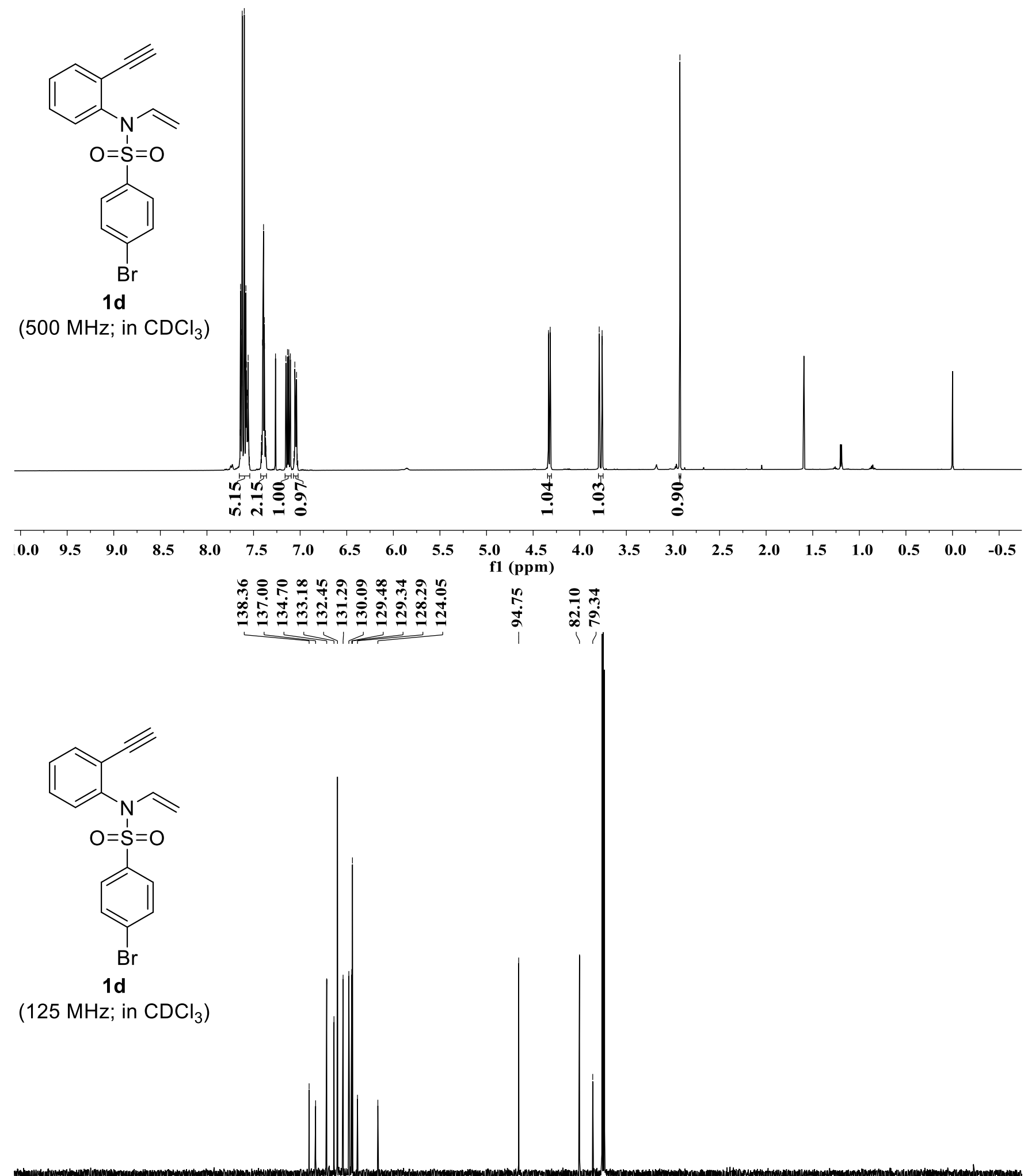




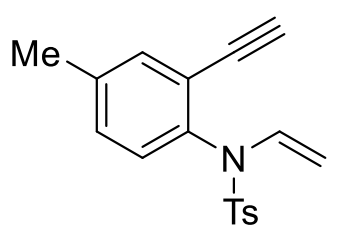

$1 \mathrm{e}$

(500 $\mathrm{MHz}$; in $\mathrm{CDCl}_{3}$ )

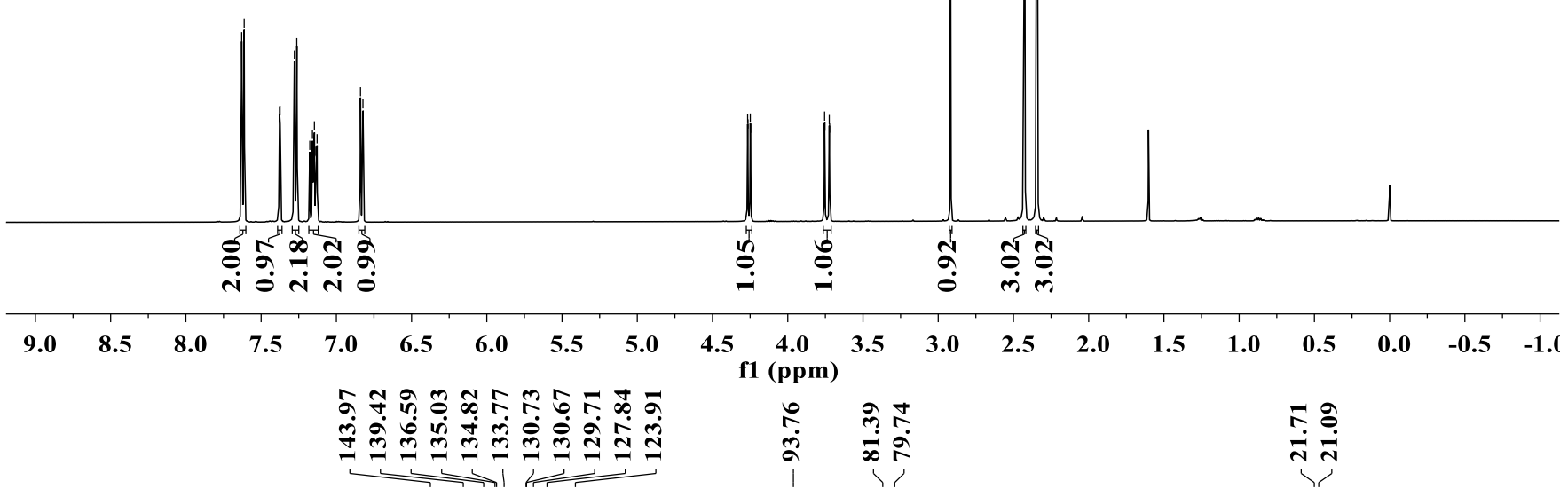<smiles>C#Cc1cc(C)ccc1N([13CH3])C=C</smiles>

$1 \mathrm{e}$

(125 $\mathrm{MHz}$; in $\mathrm{CDCl}_{3}$ ) 
<smiles>C#Cc1cc(OC)ccc1N([AsH3])C=C</smiles>

$1 f$

$\left(500 \mathrm{MHz}\right.$; in $\left.\mathrm{CDCl}_{3}\right)$

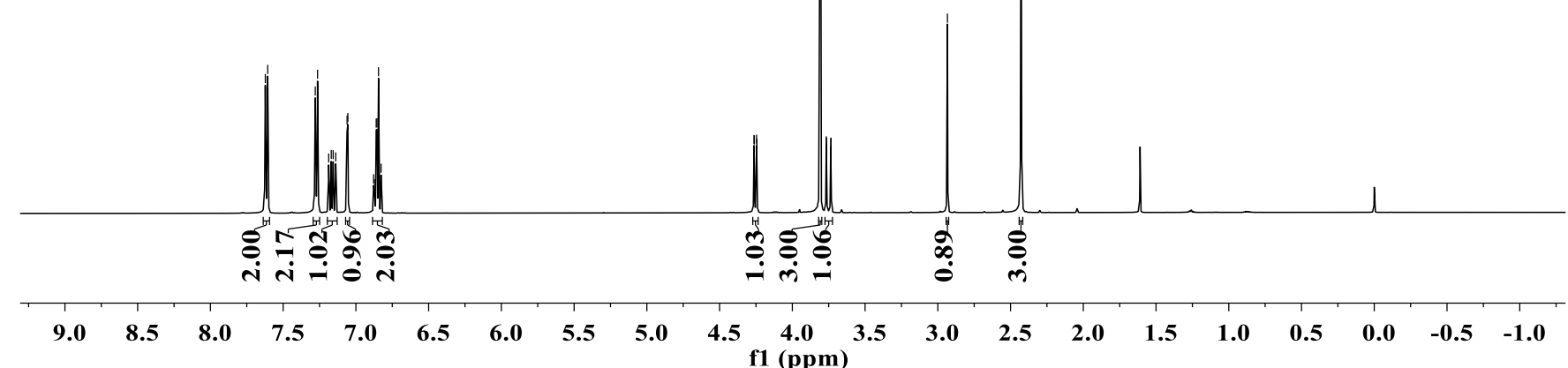<smiles>C#Cc1cc(OC)ccc1N([13CH3])C=C</smiles>

$1 f$

$\left(125 \mathrm{MHz}\right.$; in $\left.\mathrm{CDCl}_{3}\right)$

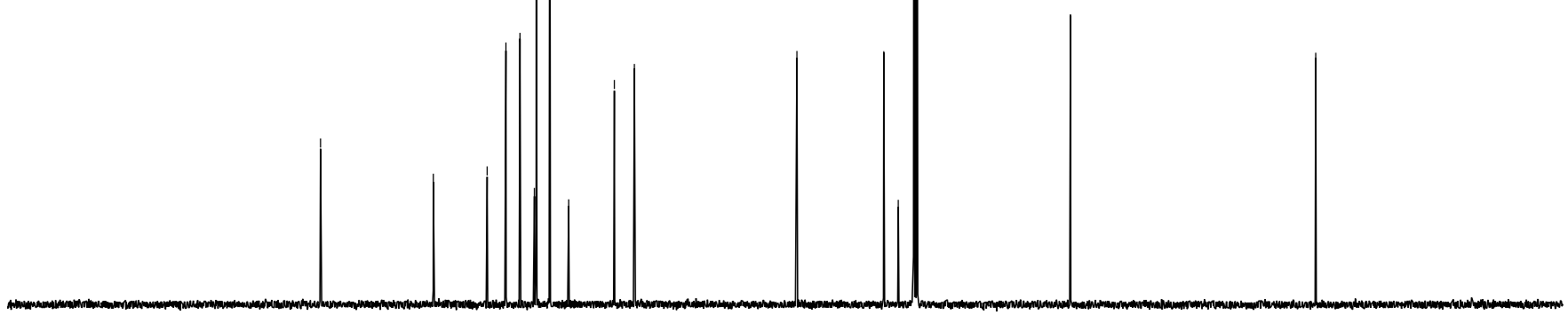




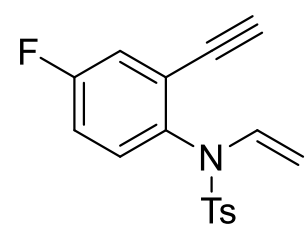

$1 \mathrm{~g}$

(500 MHz; in $\mathrm{CDCl}_{3}$ )

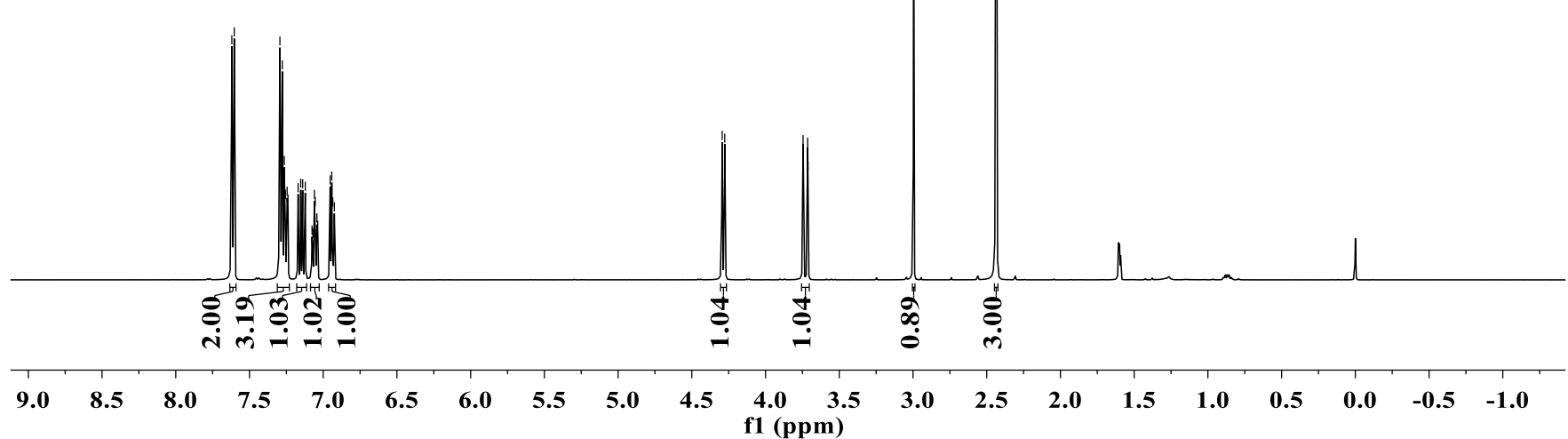

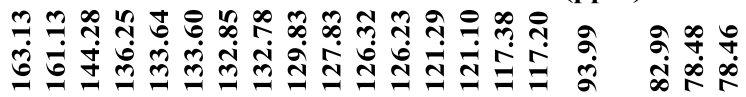

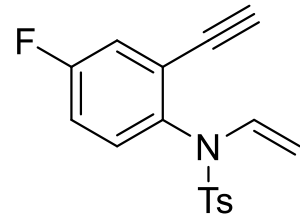

$1 \mathrm{~g}$

$\left(125 \mathrm{MHz}\right.$; in $\mathrm{CDCl}_{3}$ ) 
<smiles>C#Cc1cc(Cl)ccc1N([As])C=C</smiles>

$1 \mathrm{~h}$

$\left(500 \mathrm{MHz}\right.$; in $\mathrm{CDCl}_{3}$ )

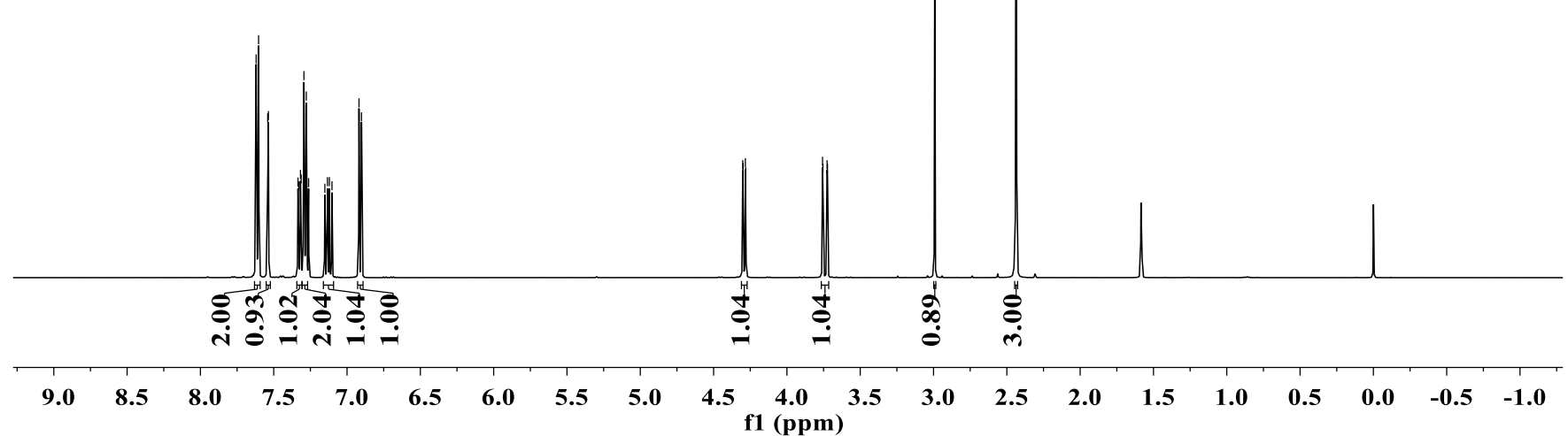

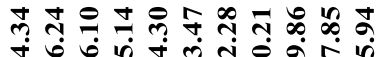

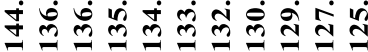<smiles>C#Cc1cc(Cl)ccc1N([As])C=C</smiles>

$1 \mathrm{~h}$

(125 MHz; in $\mathrm{CDCl}_{3}$ ) 


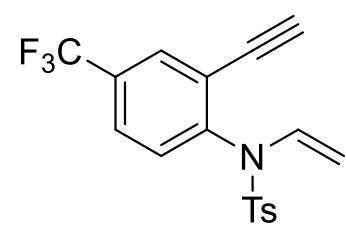

$1 \mathrm{i}$

(500 MHz; in $\mathrm{CDCl}_{3}$ )

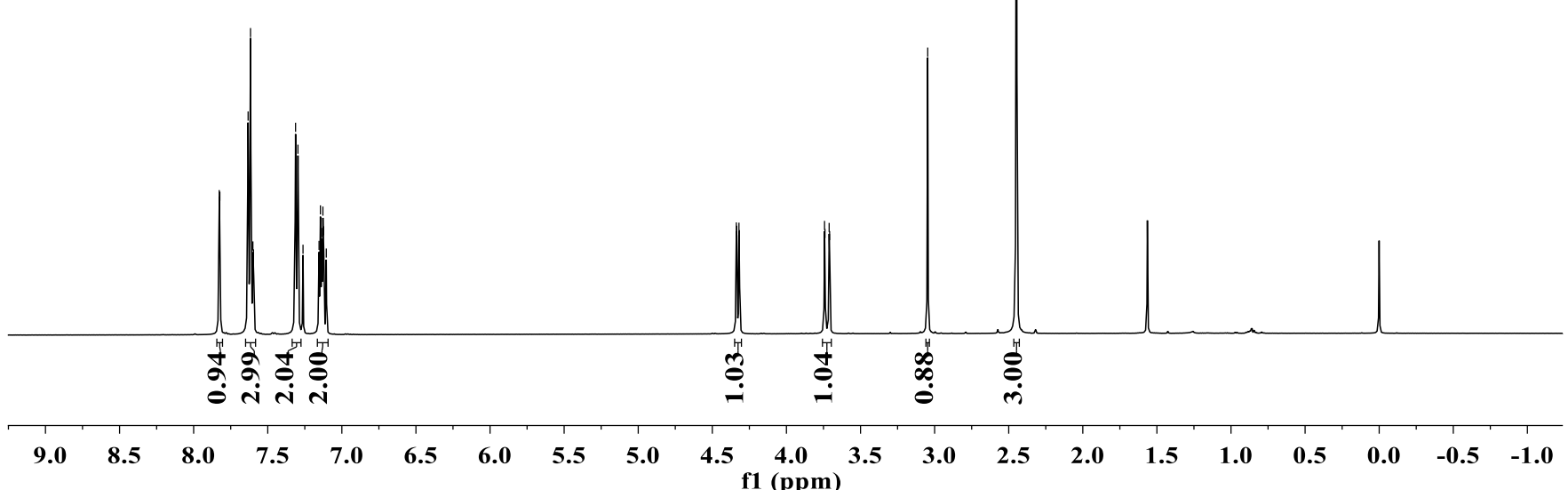

की<smiles>C#Cc1cc(C(F)(F)F)ccc1N([As])C=C</smiles>

$1 \mathrm{i}$

$\left(125 \mathrm{MHz}\right.$; in $\mathrm{CDCl}_{3}$ ) 


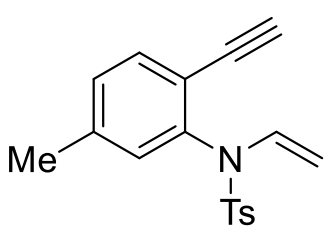

$1 \mathrm{j}$

(500 MHz; in $\mathrm{CDCl}_{3}$ )

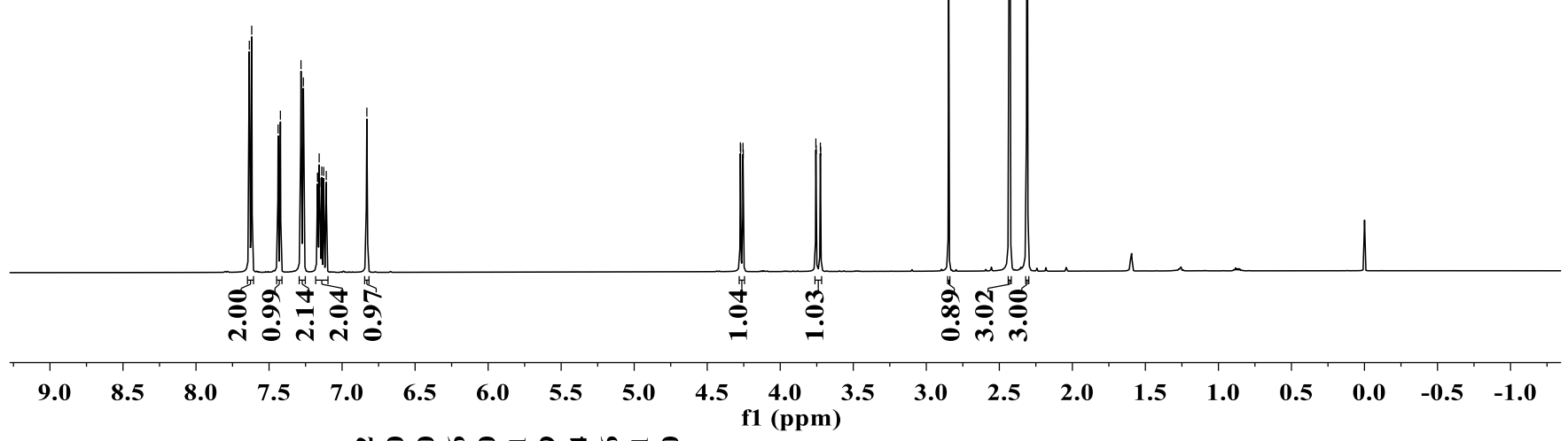

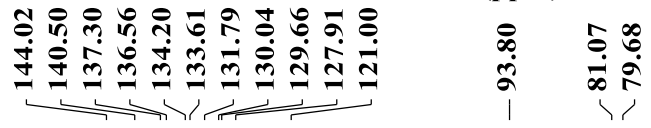

ำ<smiles>C#Cc1ccc(C)cc1N([13CH3])C=C</smiles>

$1 \mathrm{j}$

$\left(125 \mathrm{MHz}\right.$; in $\mathrm{CDCl}_{3}$ ) 
<smiles>C#Cc1ccc(OC)cc1N([13CH3])/C=C\C</smiles>

$1 \mathrm{k}$

(500 MHz; in $\mathrm{CDCl}_{3}$ )

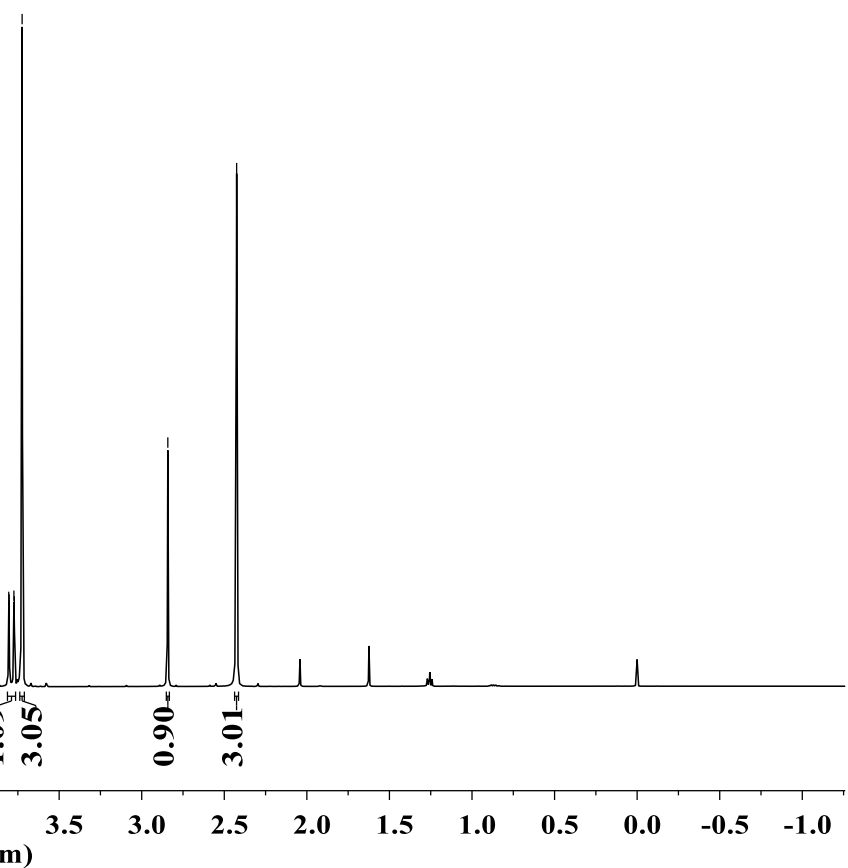

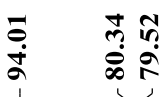

8
in
1

$\frac{a}{\text { ஸे }}$<smiles>C#Cc1ccc(OC)cc1N([13CH3])C=C</smiles>

$1 \mathrm{k}$

(125 MHz; in $\mathrm{CDCl}_{3}$ )

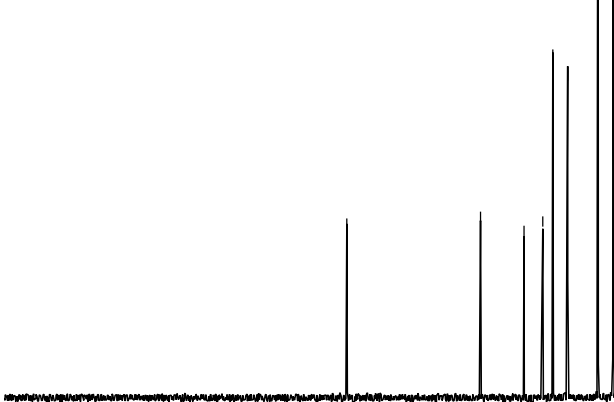


<smiles>C#Cc1ccc(F)cc1N([13F])C=C</smiles>

11

$\left(500 \mathrm{MHz}\right.$; in $\mathrm{CDCl}_{3}$ )

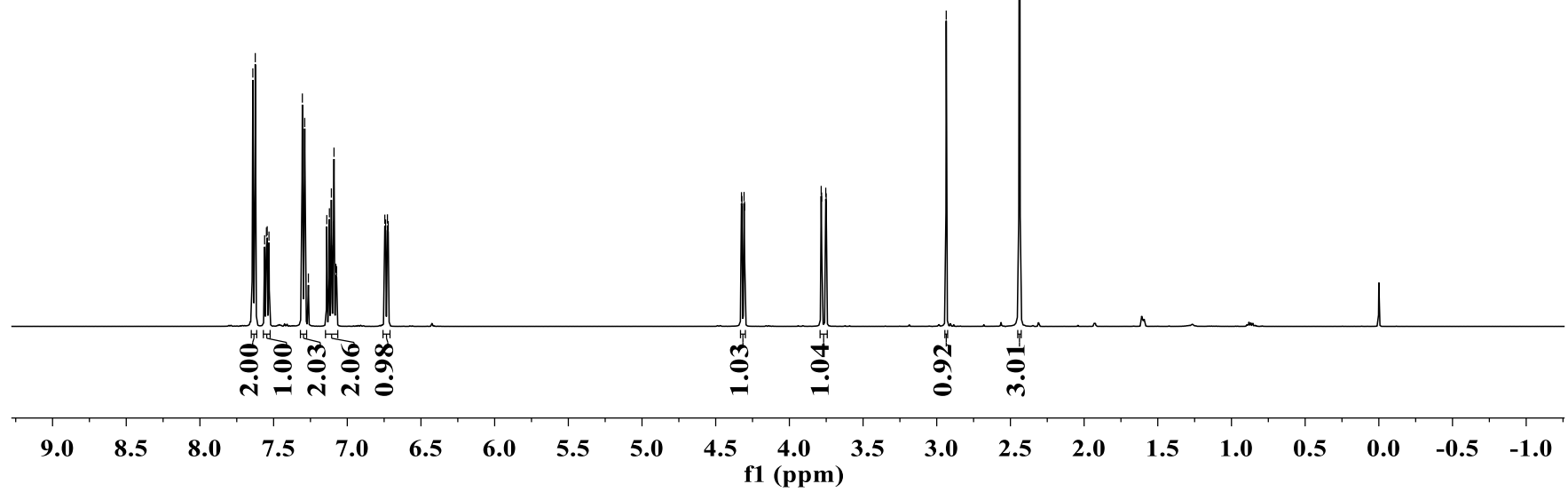

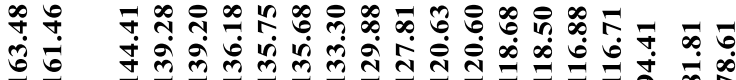

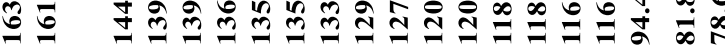<smiles>C#Cc1ccc(F)cc1N([13CH3])C=C</smiles>

11

(125 MHz; in $\mathrm{CDCl}_{3}$ ) 
<smiles>C#Cc1ccc(Cl)cc1N([As])C=C</smiles>

$1 \mathrm{~m}$

$\left(500 \mathrm{MHz}\right.$; in $\mathrm{CDCl}_{3}$ )

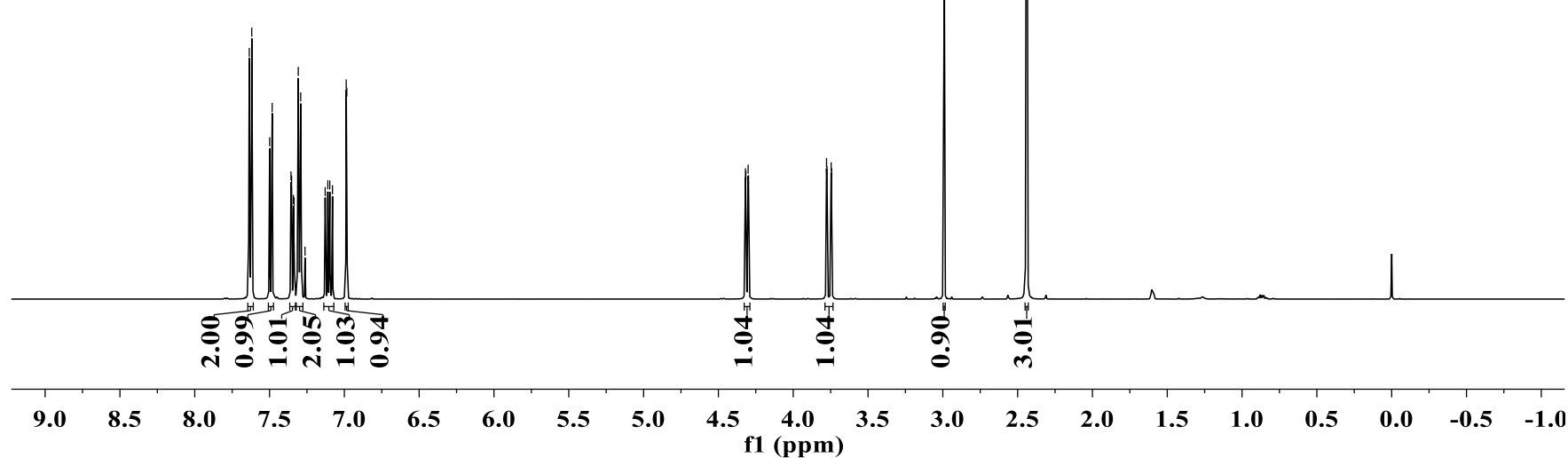

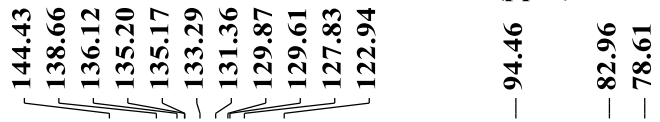<smiles>C#Cc1ccc(Cl)cc1N([As])C=C</smiles>

$1 \mathrm{~m}$

(125 MHz; in $\mathrm{CDCl}_{3}$ ) 
<smiles>[3H]N(C=C)c1cccc(C)c1C#C</smiles>

1n

$\left(500 \mathrm{MHz}\right.$; in $\mathrm{CDCl}_{3}$ )

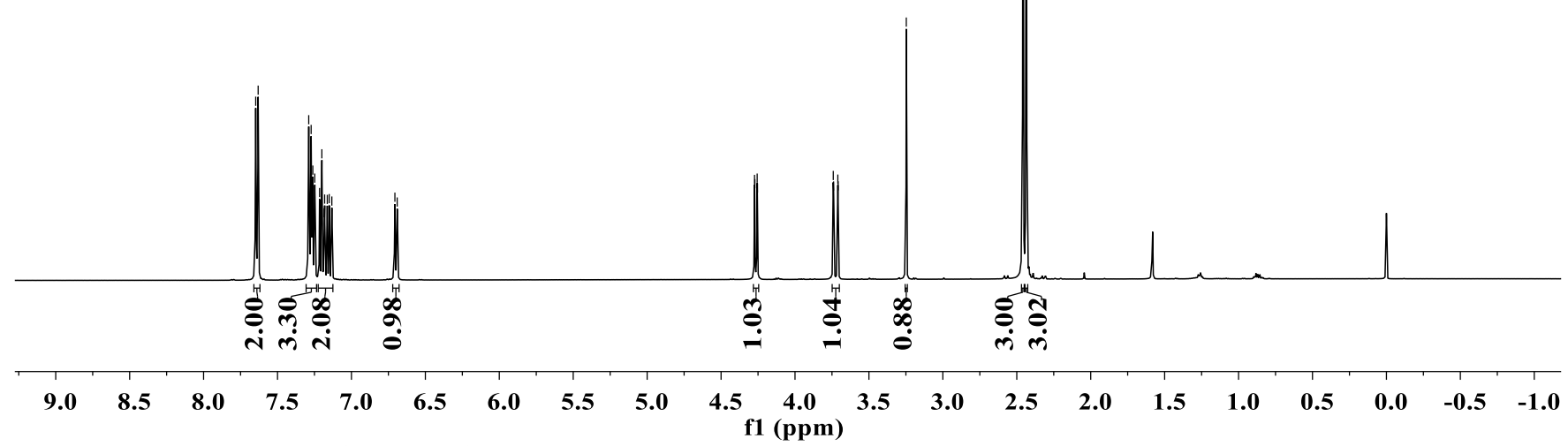

ลำ

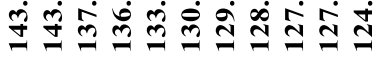

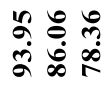

ำ<smiles>C#Cc1c(C)cccc1N([13I])C=C</smiles>

1n

(125 MHz; in $\mathrm{CDCl}_{3}$ ) 


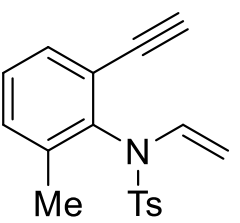

10

$\left(500 \mathrm{MHz}\right.$; in $\mathrm{CDCl}_{3}$ )

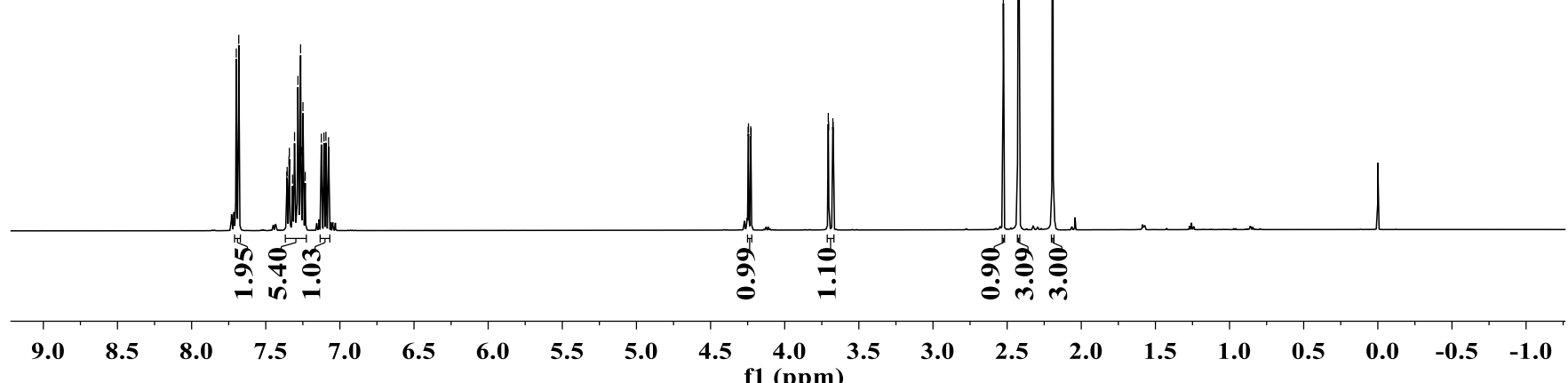

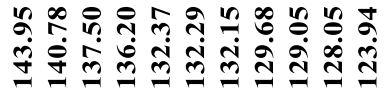

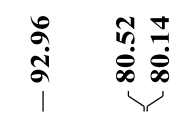

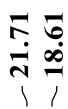<smiles>C#Cc1cccc(C)c1N([13CH3])C=C</smiles>

10

$\left(125 \mathrm{MHz}\right.$; in $\mathrm{CDCl}_{3}$ ) 


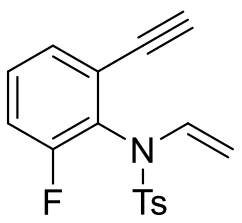

$1 \mathrm{p}$

(500 MHz; in $\mathrm{CDCl}_{3}$ )

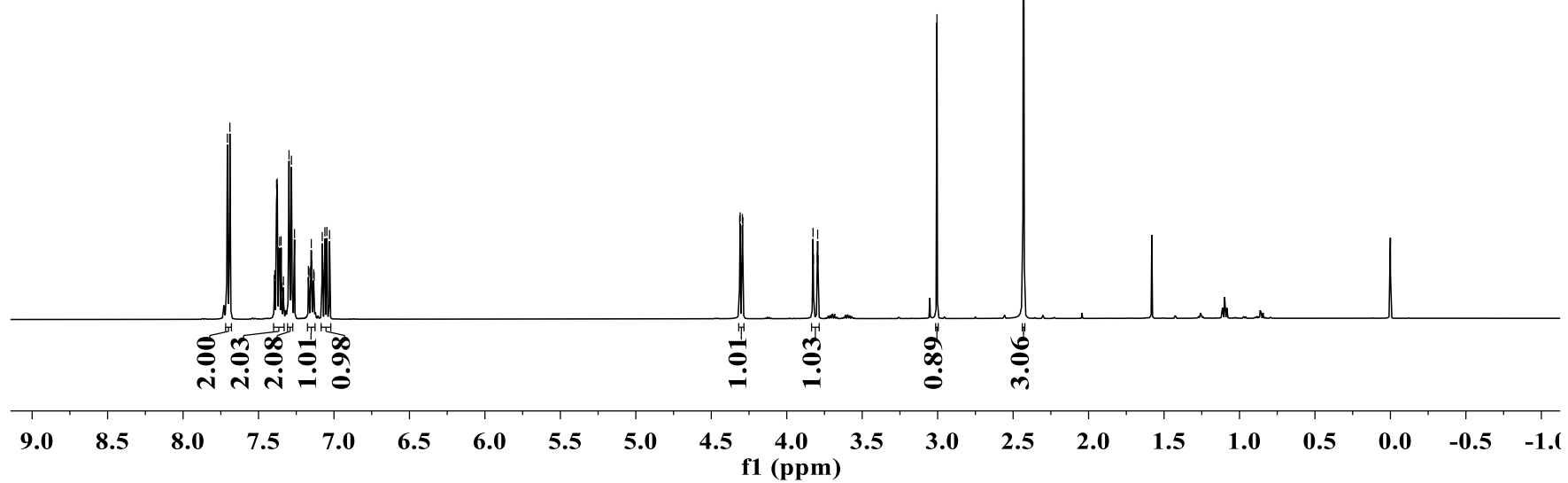

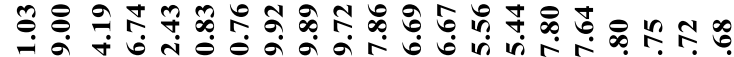

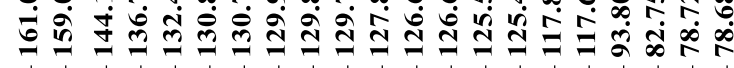

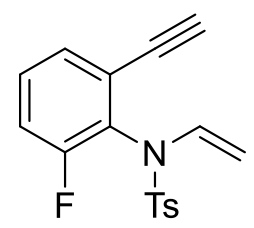

$1 \mathrm{p}$

$\left(125 \mathrm{MHz}\right.$; in $\mathrm{CDCl}_{3}$ ) 


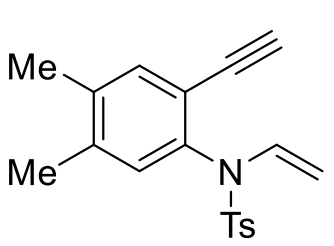

$1 q$

(500 MHz; in $\mathrm{CDCl}_{3}$ )

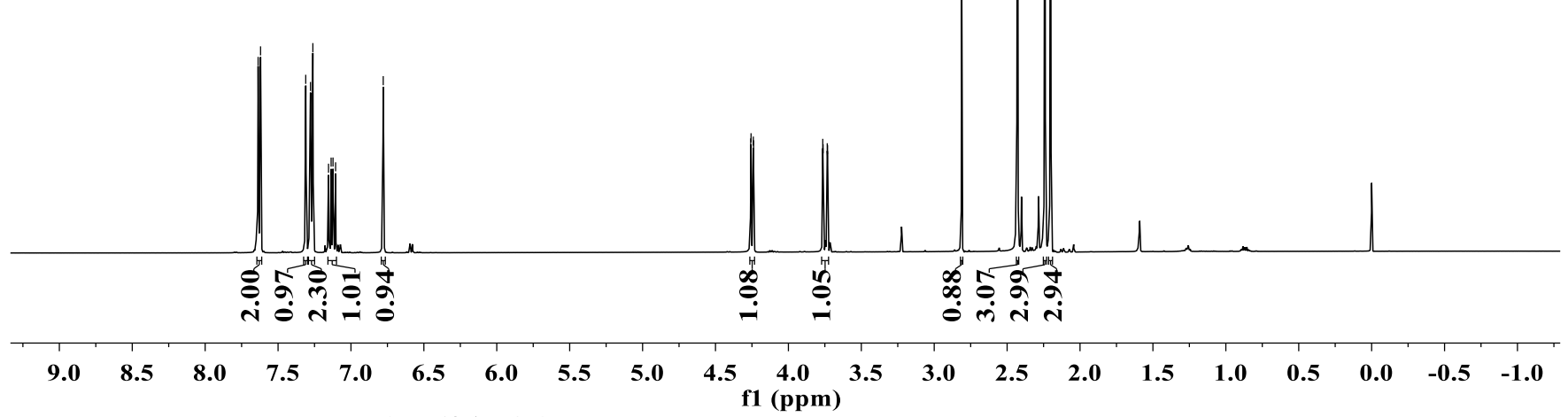

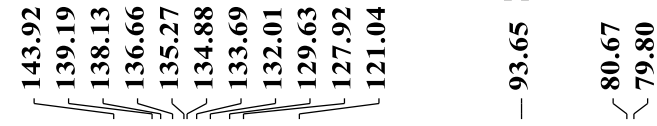

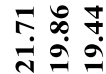<smiles>C#Cc1cc(C)c(C)cc1N([13CH3])C=C</smiles>

19

(125 MHz; in $\mathrm{CDCl}_{3}$ ) 


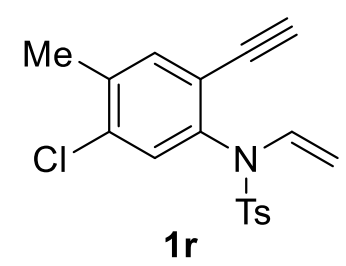

(500 MHz; in $\mathrm{CDCl}_{3}$ )

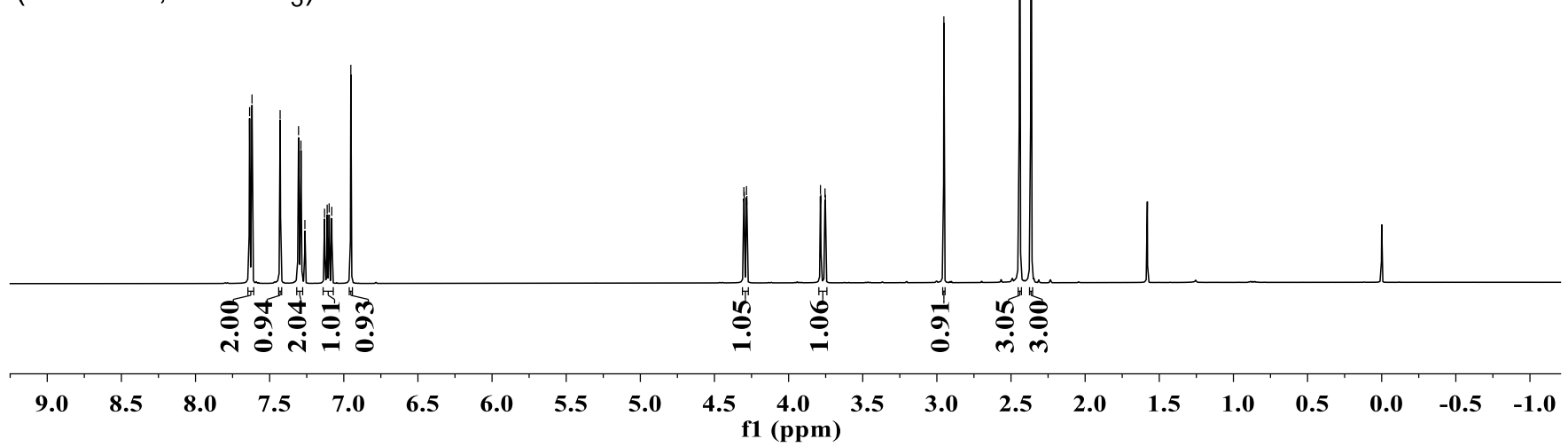

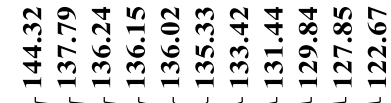

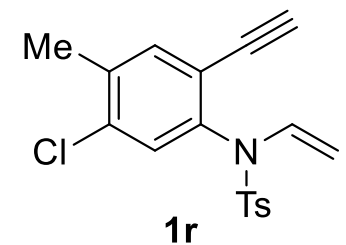

(125 MHz; in $\mathrm{CDCl}_{3}$ ) 
<smiles>C#Cc1cc(F)cc(C)c1N([13F])C=C</smiles>

$1 \mathrm{~s}$

(500 MHz; in $\mathrm{CDCl}_{3}$ )

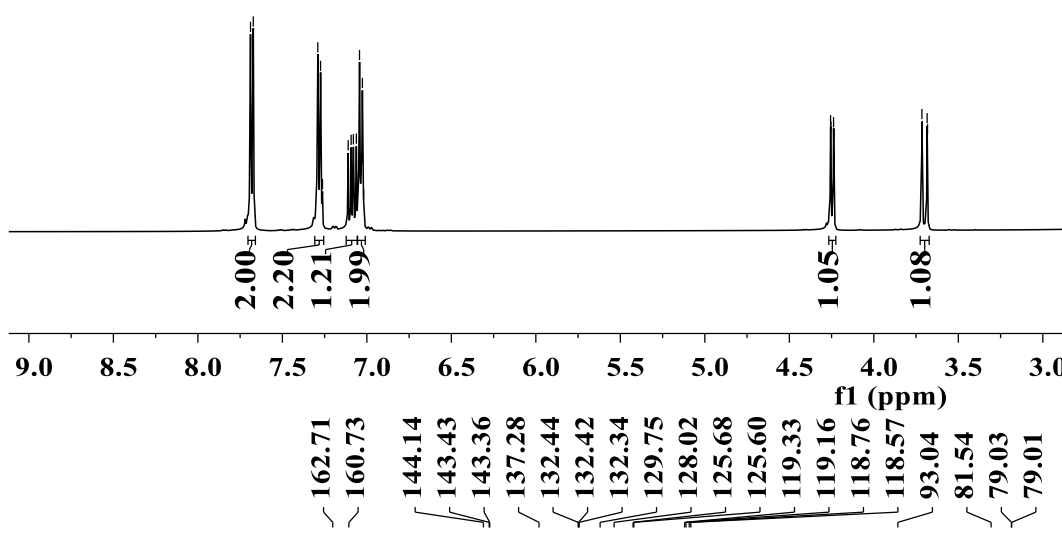

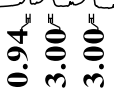

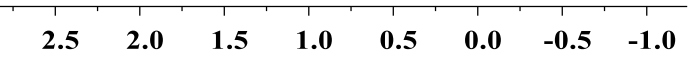

ํㅗㅇ<smiles>C#Cc1cc(F)cc(C)c1N([125I])C=C</smiles>

$1 \mathrm{~s}$

(125 MHz; in $\mathrm{CDCl}_{3}$ ) 


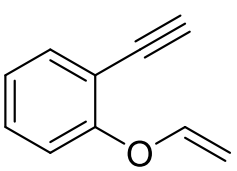

$1 \mathbf{t}$

$\left(500 \mathrm{MHz}\right.$; in $\left.\mathrm{CDCl}_{3}\right)$

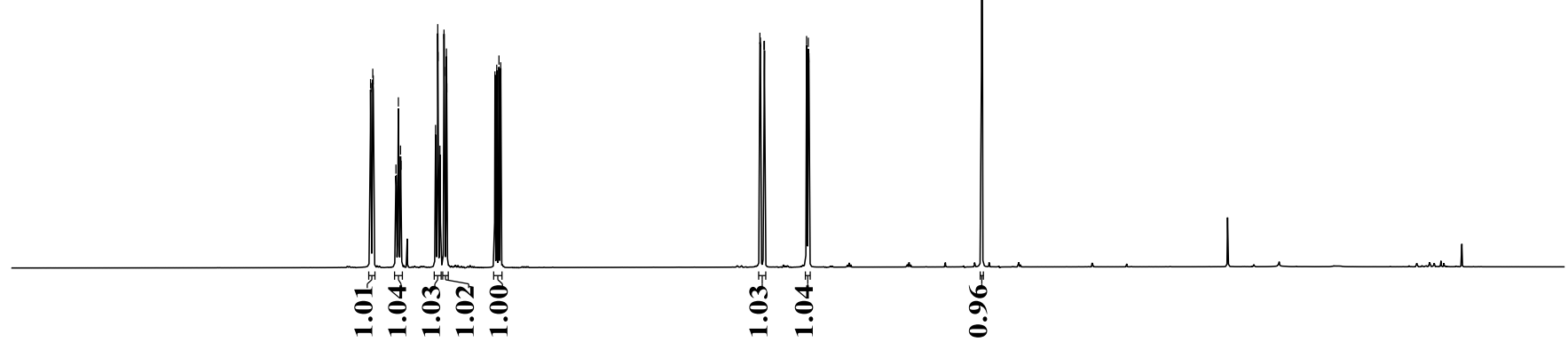

$\begin{array}{llllllllllllllllllllllll}9.5 & 9.0 & 8.5 & 8.0 & 7.5 & 7.0 & 6.5 & 6.0 & 5.5 & \begin{array}{c}5.0 \\ \text { f1 }(\mathrm{ppm})\end{array} & 4.5 & 4.0 & 3.5 & 3.0 & 2.5 & 2.0 & 1.5 & 1.0 & 0.5 & 0.0 & -0.5\end{array}$

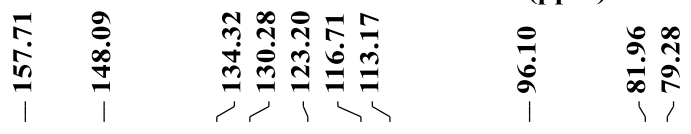<smiles>C#Cc1ccccc1OC=C</smiles>

$1 \mathbf{t}$

$\left(125 \mathrm{MHz}\right.$; in $\left.\mathrm{CDCl}_{3}\right)$ 


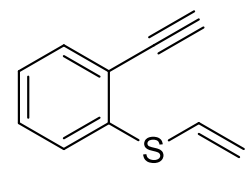

1u

$\left(500 \mathrm{MHz}\right.$; in $\left.\mathrm{CDCl}_{3}\right)$

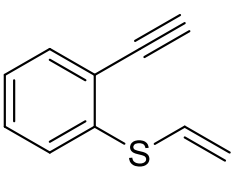

1u

$\left(125 \mathrm{MHz}\right.$; in $\mathrm{CDCl}_{3}$ )

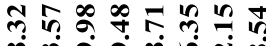

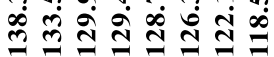

i $\infty$

hel

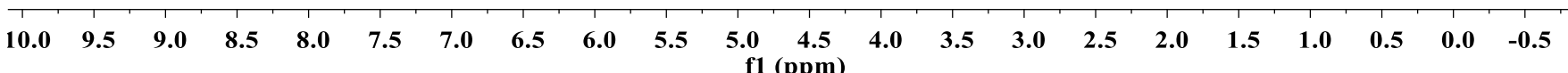

f1 (ppm)

\section{ஸे}

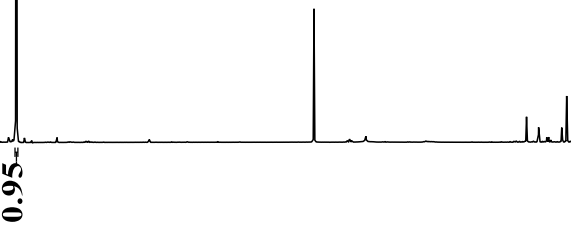




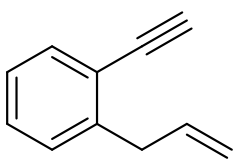

$1 v$

$\left(500 \mathrm{MHz}\right.$; in $\left.\mathrm{CDCl}_{3}\right)$

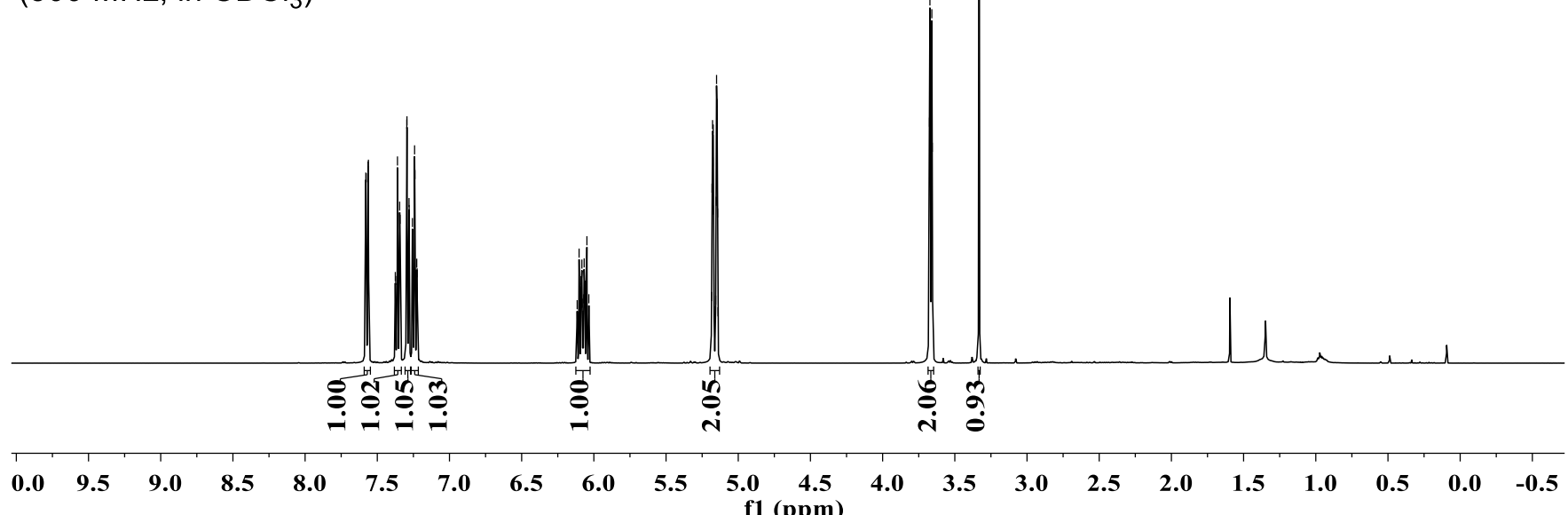

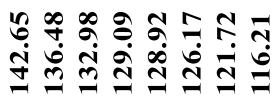

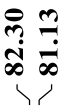

$\underset{\infty}{\infty}$

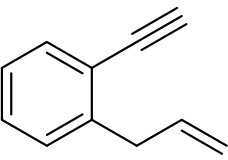

$1 v$

$\left(125 \mathrm{MHz}\right.$; in $\left.\mathrm{CDCl}_{3}\right)$ 
<smiles>C#CCCN([13F])C=C</smiles>

$1 w$

(500 MHz; in $\mathrm{CDCl}_{3}$ )

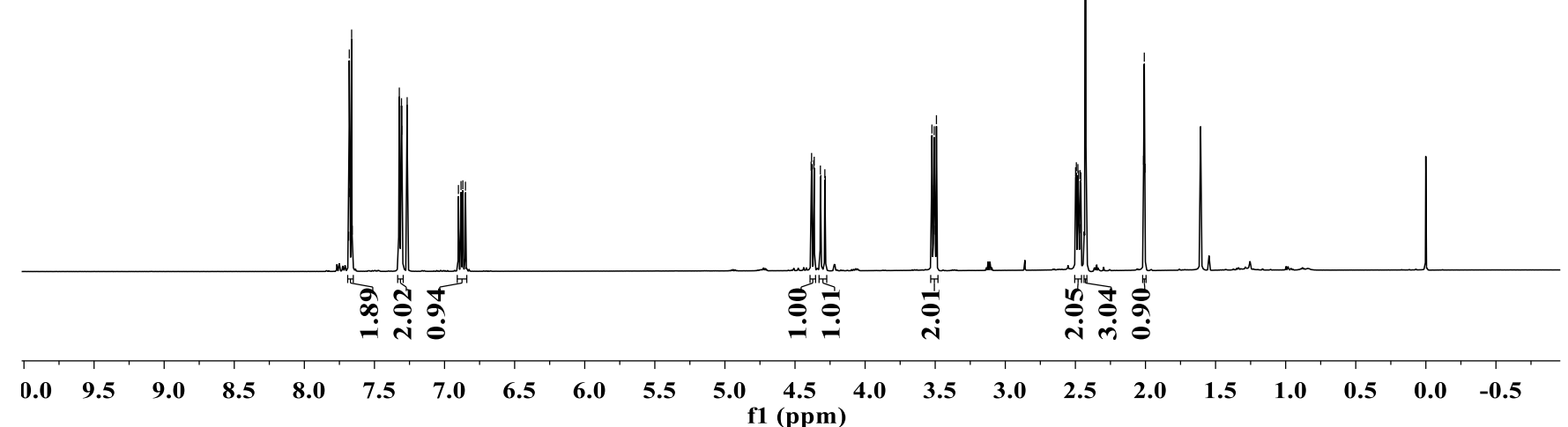

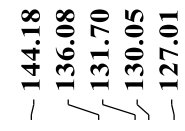

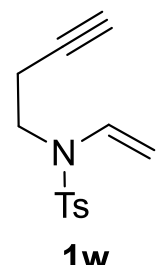

$\left(125 \mathrm{MHz}\right.$; in $\mathrm{CDCl}_{3}$ ) 


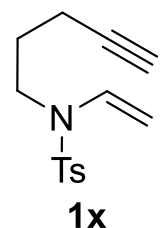

$\left(500 \mathrm{MHz}\right.$; in $\mathrm{CDCl}_{3}$ )

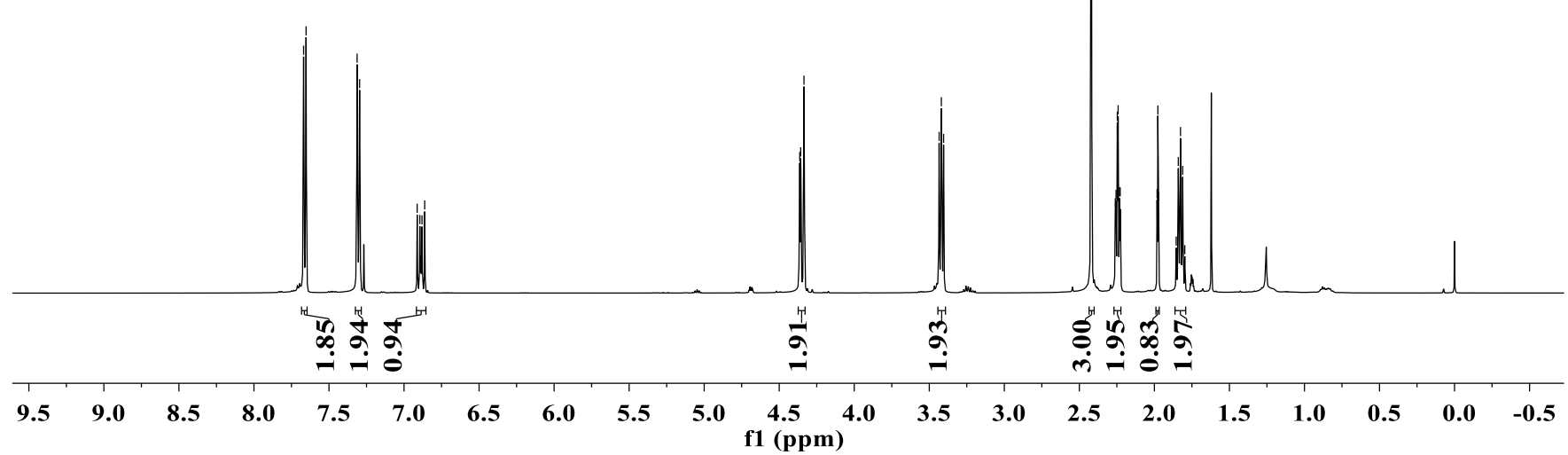

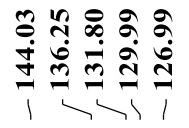<smiles>C#CCCCN(C)C=C</smiles>

$1 \mathbf{x}$

$\left(125 \mathrm{MHz}\right.$; in $\left.\mathrm{CDCl}_{3}\right)$ 
<smiles>C#Cc1ccccc1N([As])/C=C/C</smiles>

$(E)-1 \mathbf{y}$

$\left(500 \mathrm{MHz}\right.$; in $\mathrm{CDCl}_{3}$ )

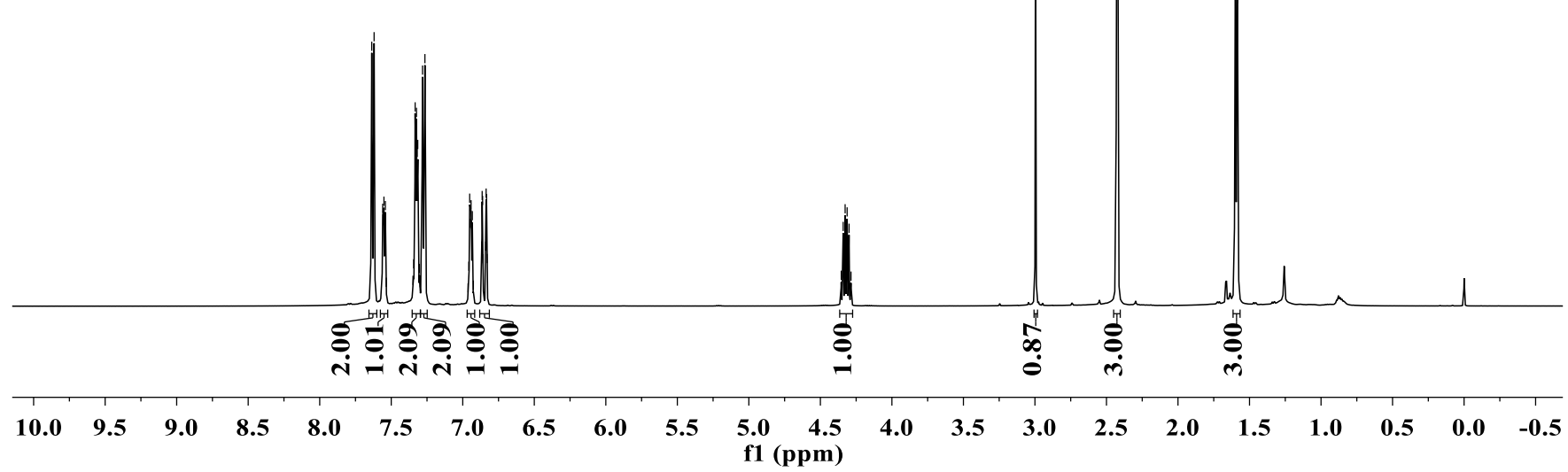

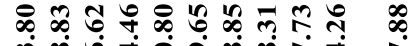

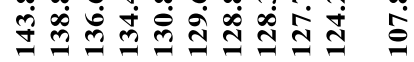

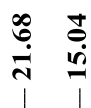<smiles>C#Cc1ccccc1N([As])/C=C/C</smiles>

$(E)-1 \mathbf{y}$

$\left(125 \mathrm{MHz}\right.$; in $\left.\mathrm{CDCl}_{3}\right)$

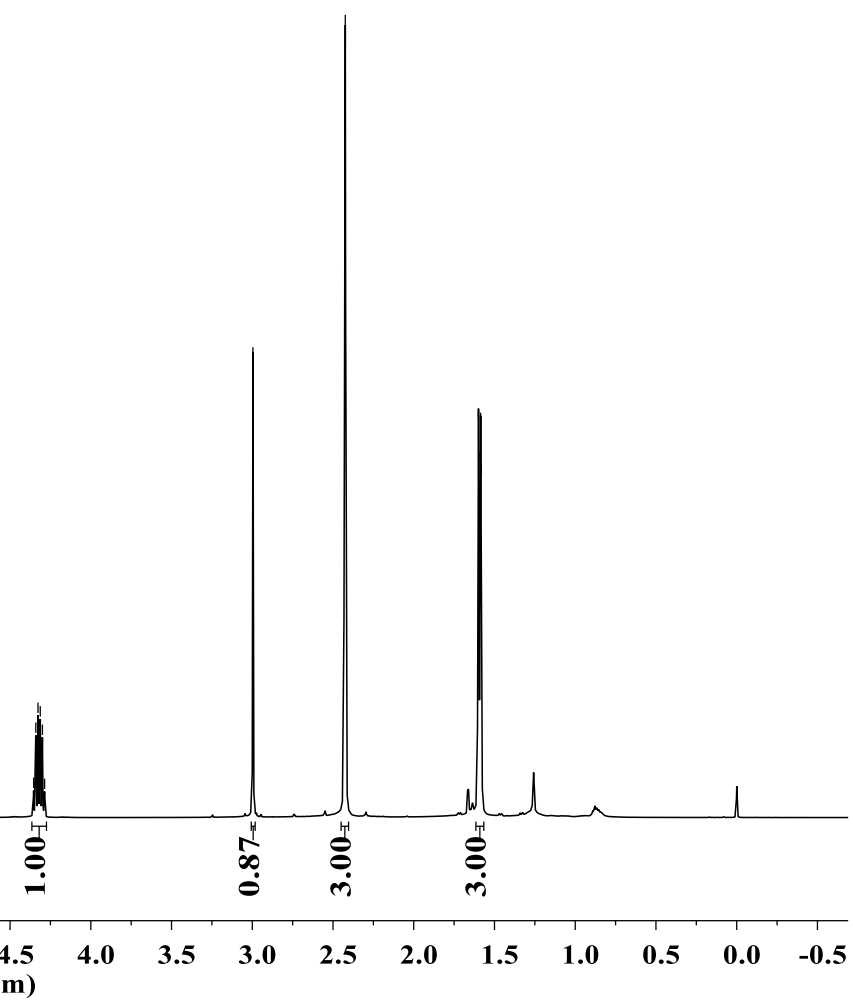

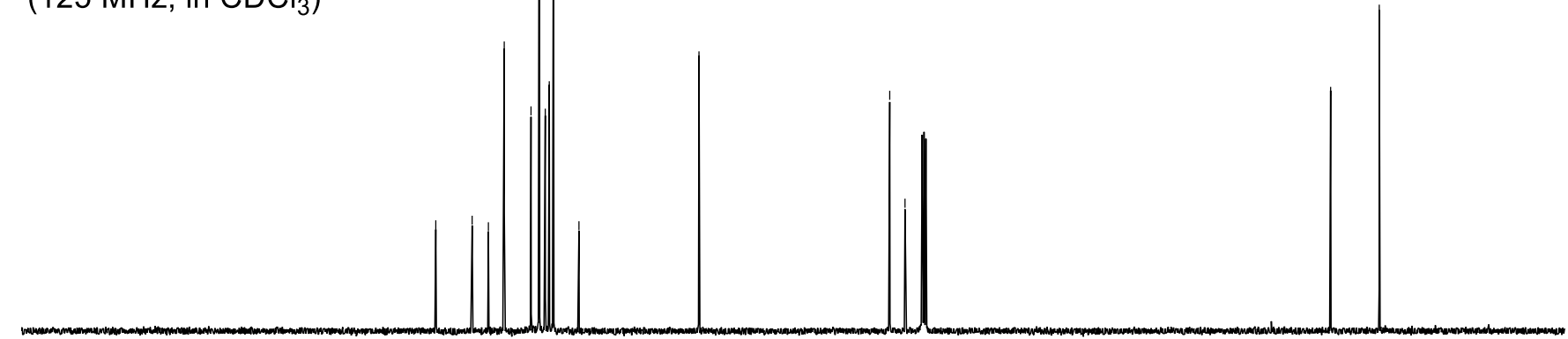

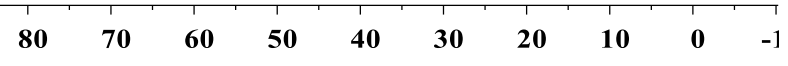




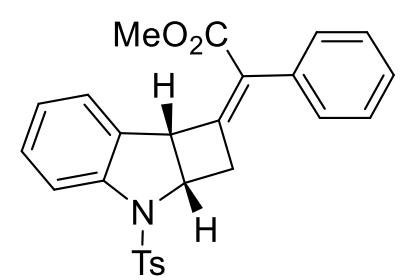

(Z)-3aa

$\left(500 \mathrm{MHz}\right.$; in $\left.\mathrm{CDCl}_{3}\right)$

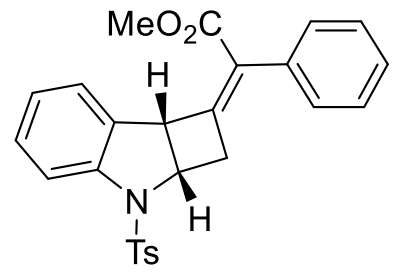

(Z)-3aa

$\left(125 \mathrm{MHz}\right.$; in $\left.\mathrm{CDCl}_{3}\right)$ 


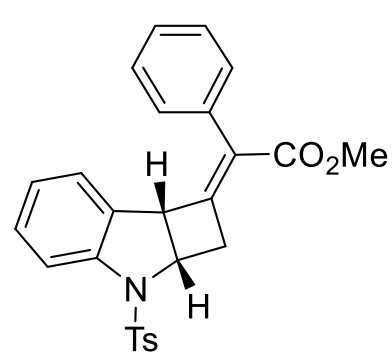

(E)-3aa

$\left(500 \mathrm{MHz}\right.$; in $\left.\mathrm{CDCl}_{3}\right)$

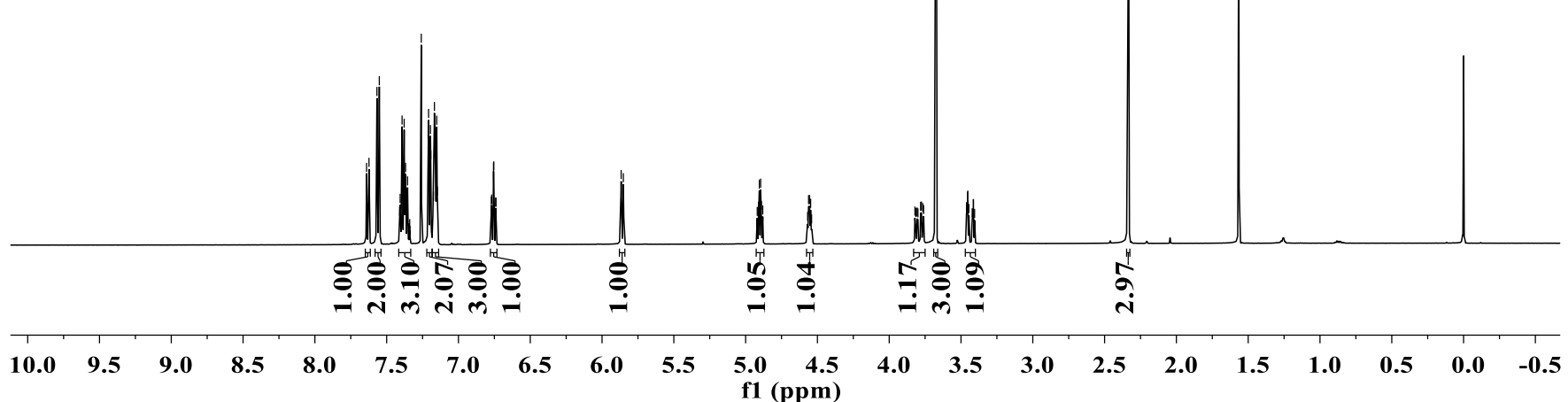

苟

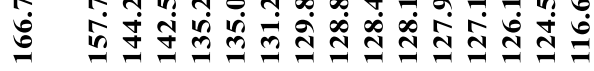

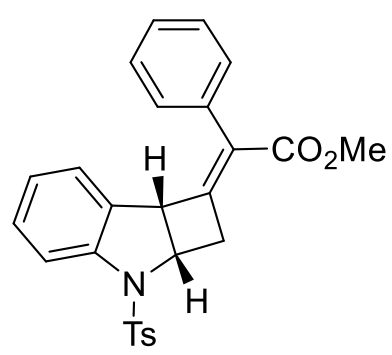

(E)-3aa

(125 $\mathrm{MHz}$; in $\mathrm{CDCl}_{3}$ ) 


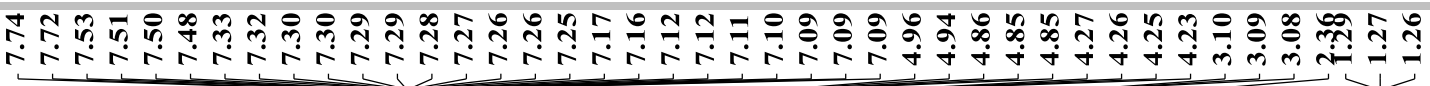

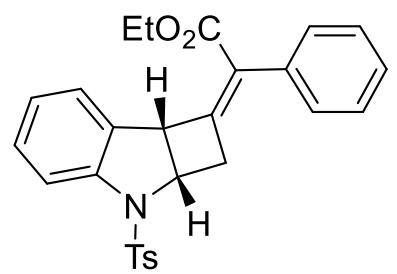

(Z)-3ab

$\left(500 \mathrm{MHz}\right.$; in $\left.\mathrm{CDCl}_{3}\right)$

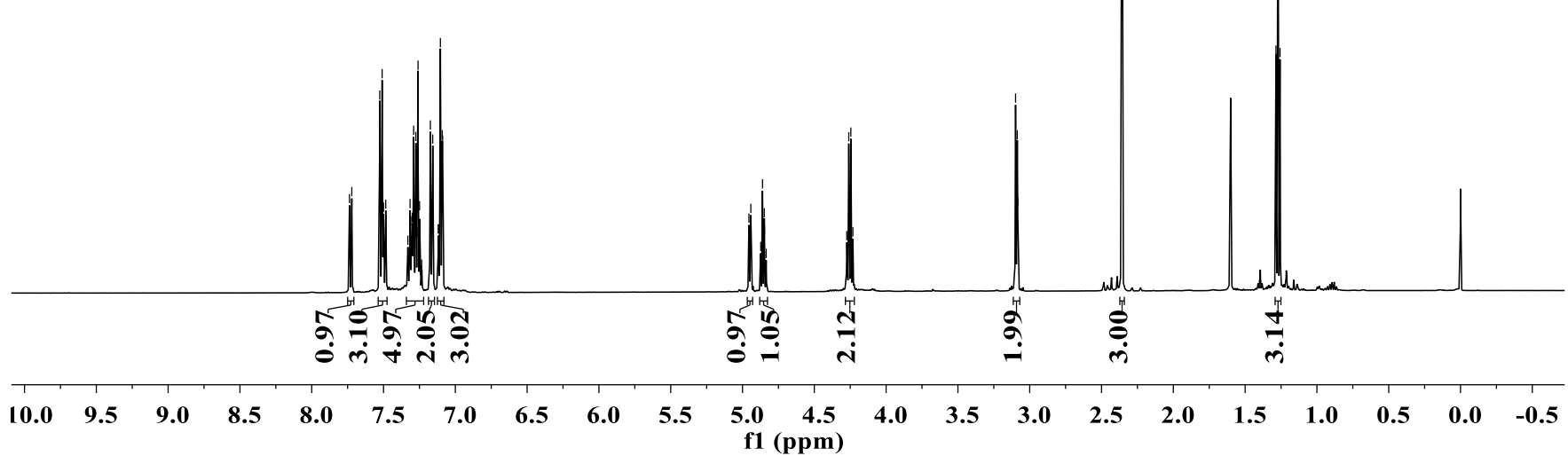

n.

苟

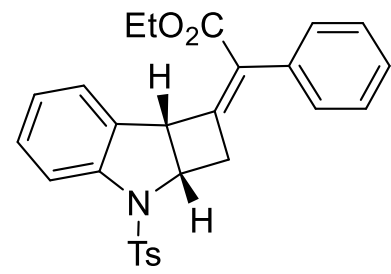

(Z)-3ab

$\left(125 \mathrm{MHz}\right.$; in $\mathrm{CDCl}_{3}$ )

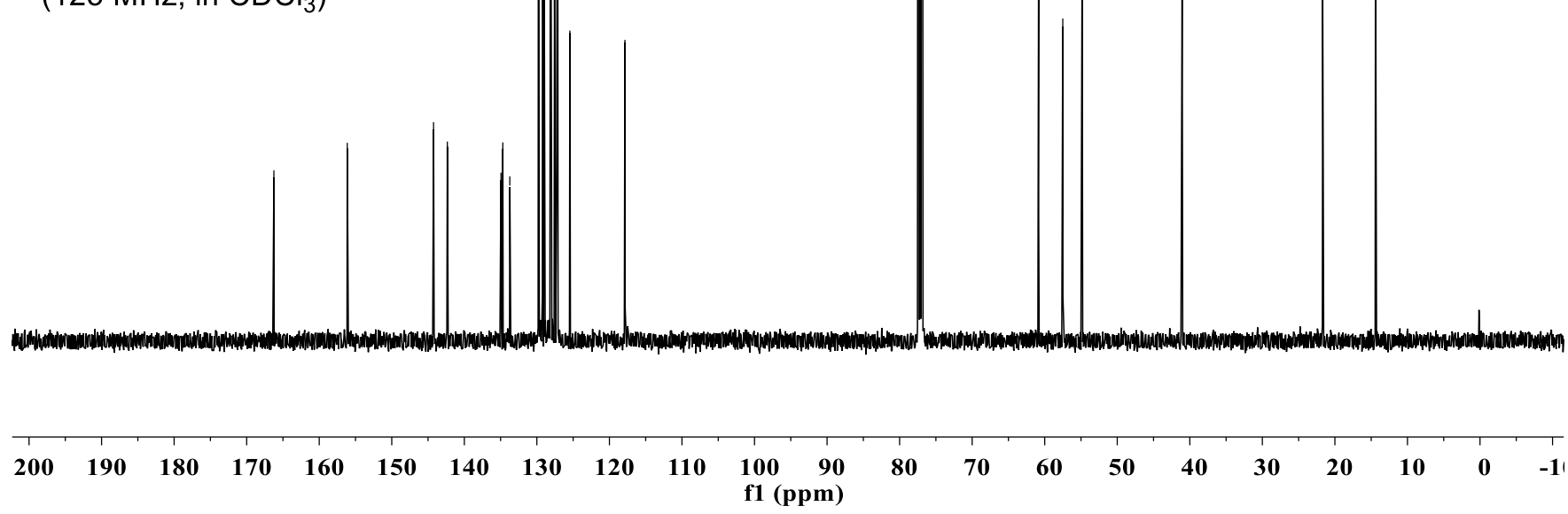




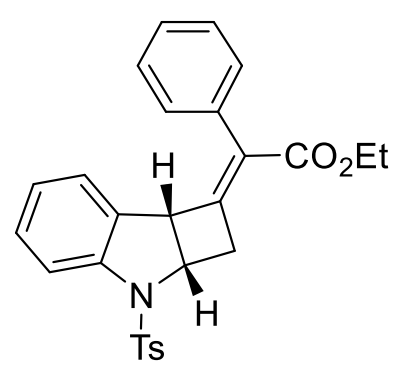

$(E)-3 \mathbf{a b}$

(500 MHz; in $\mathrm{CDCl}_{3}$ )

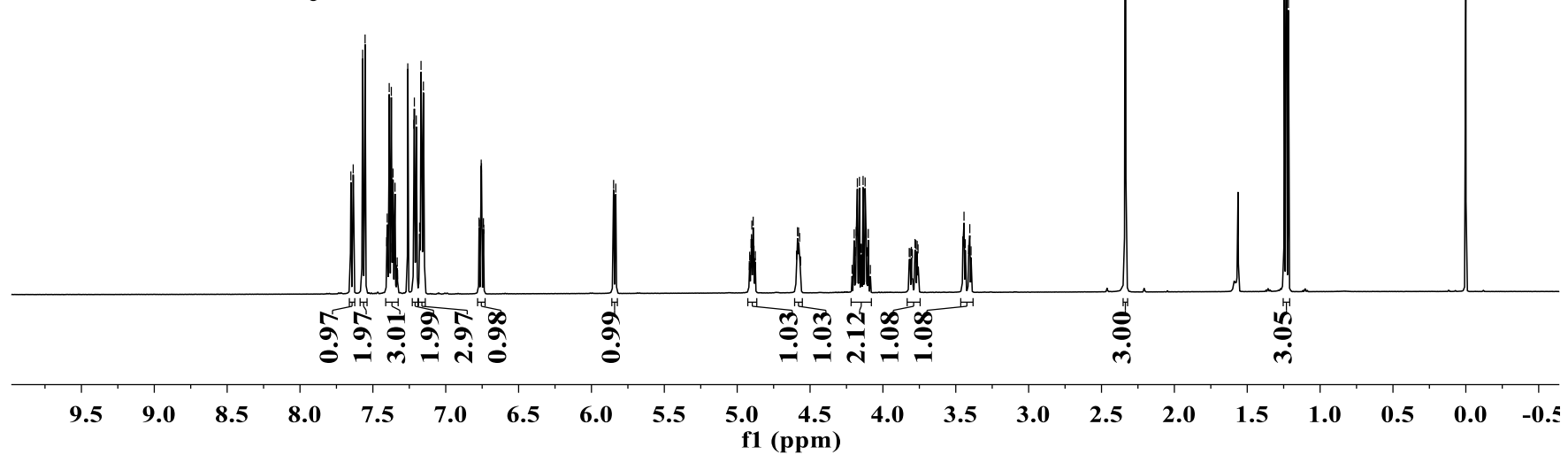

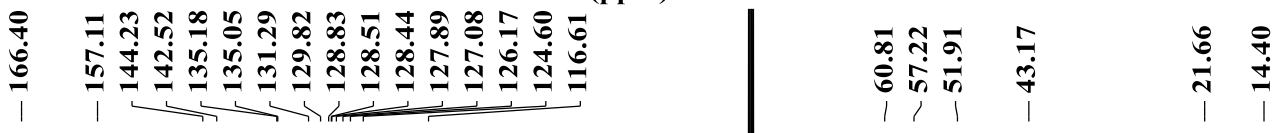

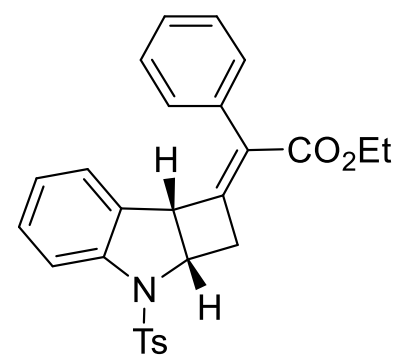

(E)-3ab

(125 MHz; in $\mathrm{CDCl}_{3}$ ) 


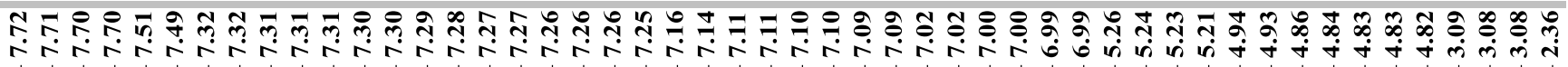

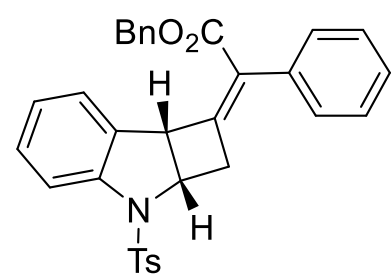

(Z)-3ac

$\left(500 \mathrm{MHz}\right.$; in $\left.\mathrm{CDCl}_{3}\right)$

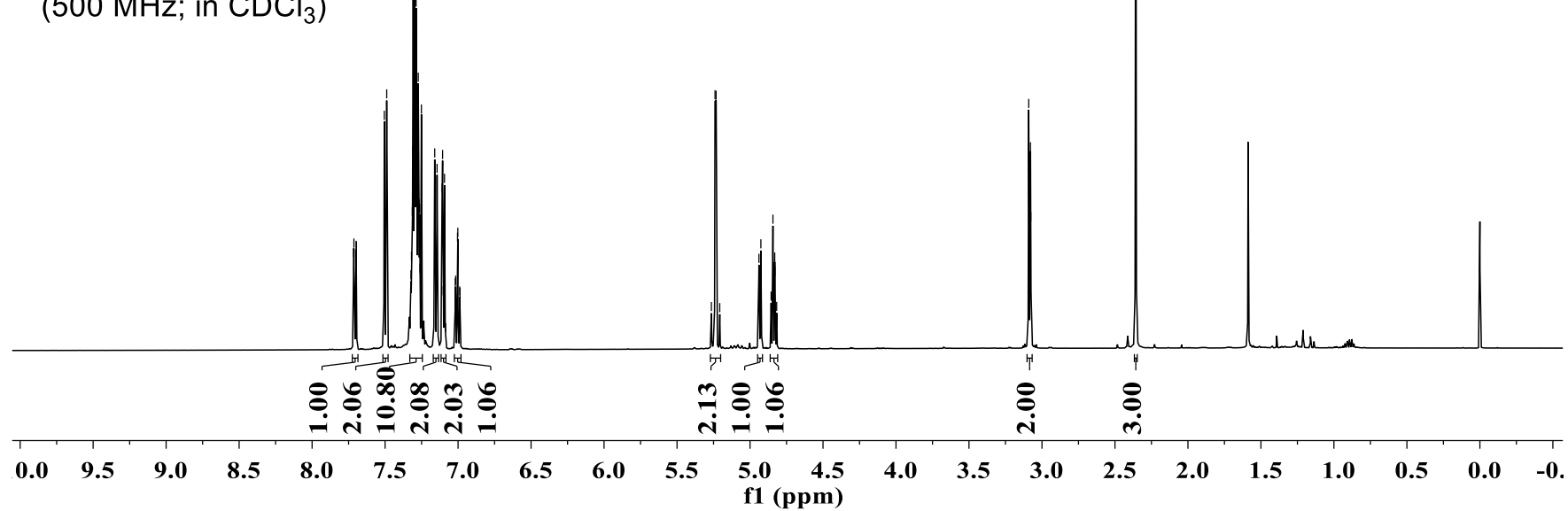

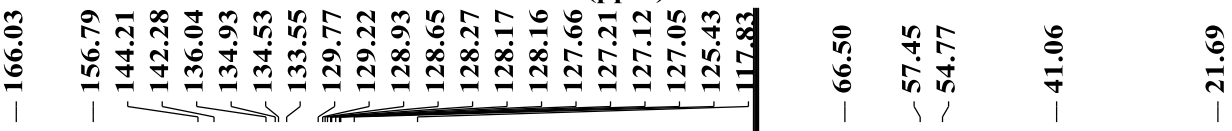

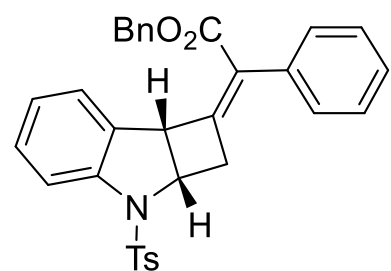

(Z)-3ac

$\left(125 \mathrm{MHz}\right.$; in $\mathrm{CDCl}_{3}$ ) 


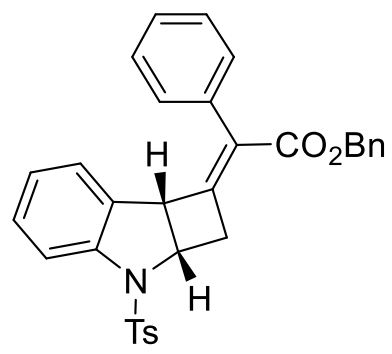

(E)-3ac

$\left(500 \mathrm{MHz}\right.$; in $\left.\mathrm{CDCl}_{3}\right)$

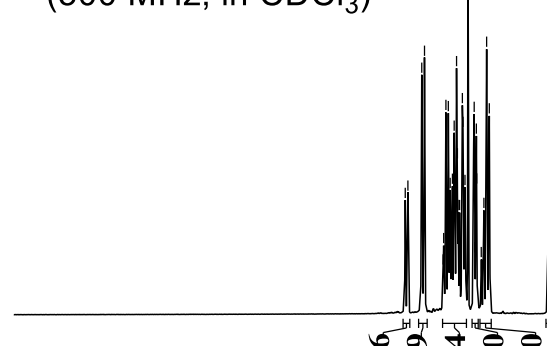

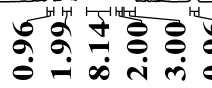

$0.0 \quad 9.5 \quad 9.0$

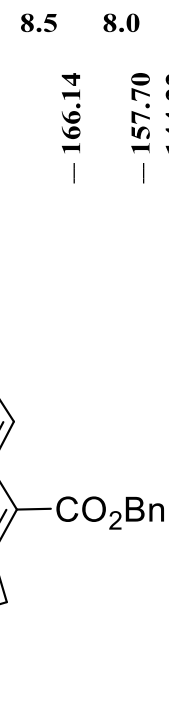

(E)-3ac

(125 MHz; in $\mathrm{CDCl}_{3}$ )

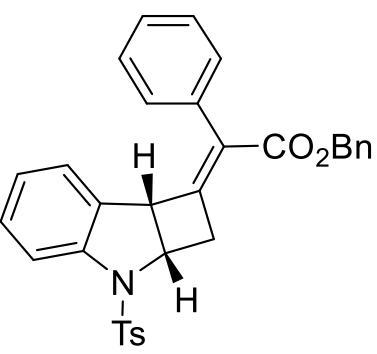

$0 \infty a \infty$

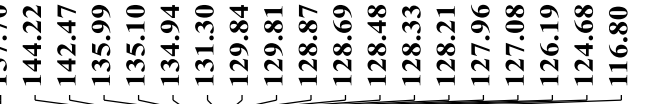

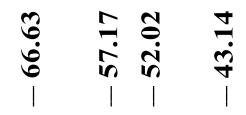

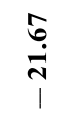




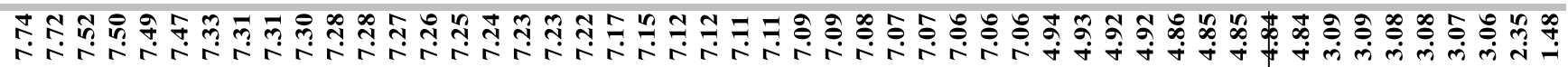

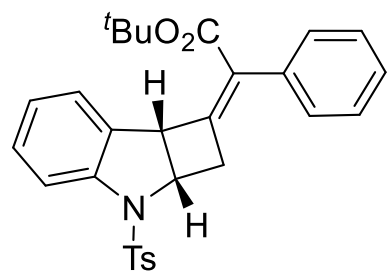

(Z)-3ad

$\left(500 \mathrm{MHz}\right.$; in $\mathrm{CDCl}_{3}$ )

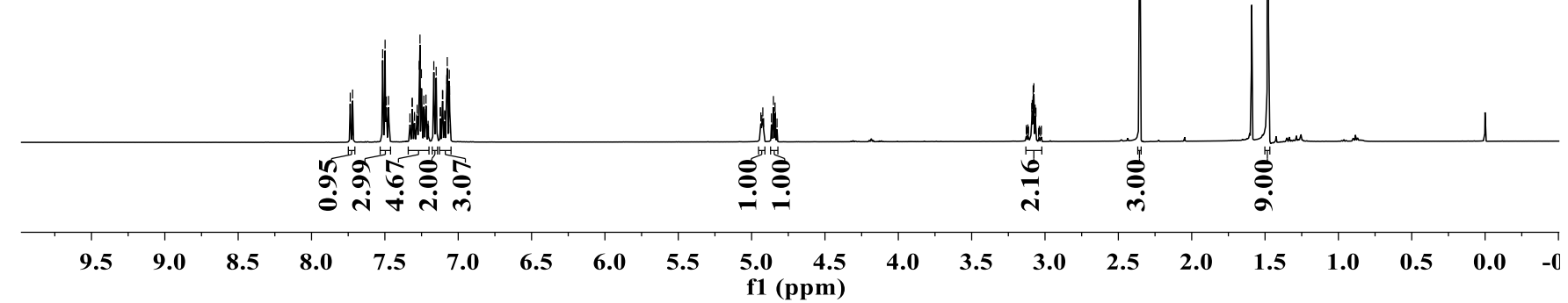

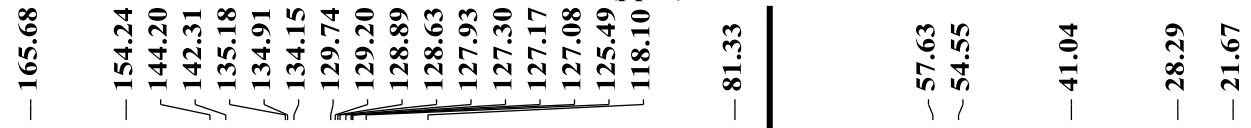

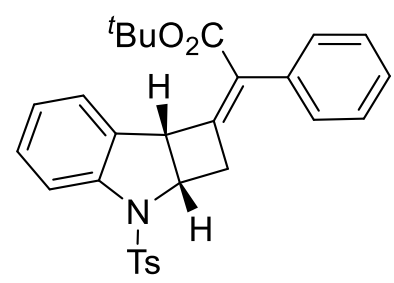

(Z)-3ad

$\left(125 \mathrm{MHz}\right.$; in $\mathrm{CDCl}_{3}$ )

$\begin{array}{lllllllllll}190 & 180 & 170 & 160 & 150 & 140 & 130 & 120 & 110 & 100 & 90\end{array}$

$\begin{array}{llllllllll}80 & 70 & 60 & 50 & 40 & 30 & 20 & 10 & 0 & -1\end{array}$ f1 (ppm) 


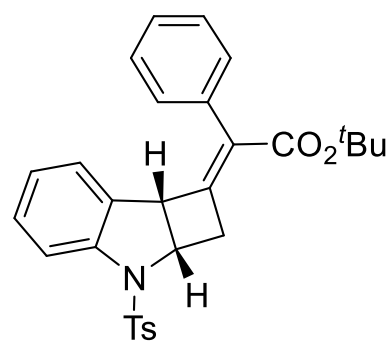

$(E)-3 a d$

$\left(500 \mathrm{MHz}\right.$; in $\left.\mathrm{CDCl}_{3}\right)$

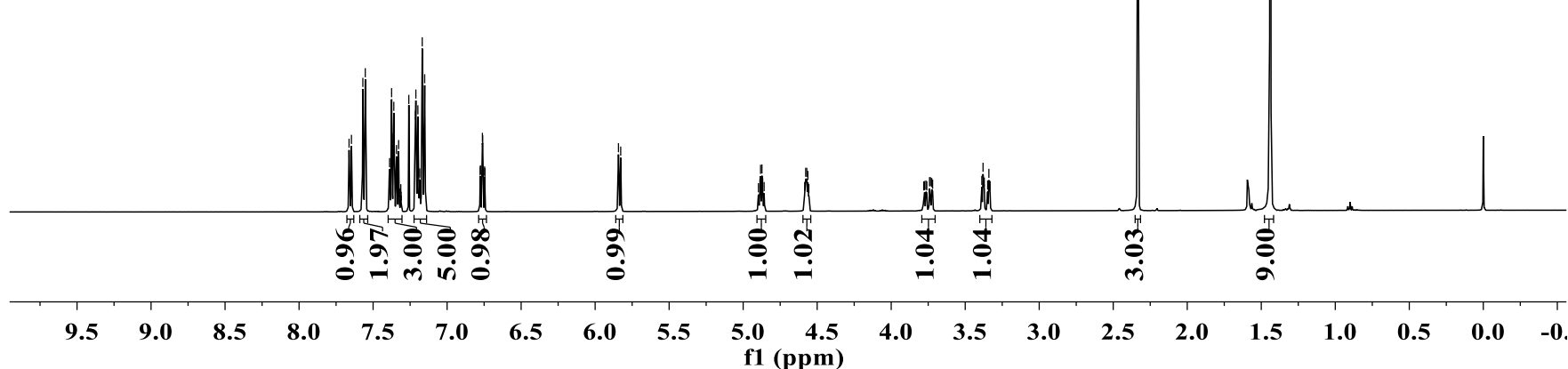

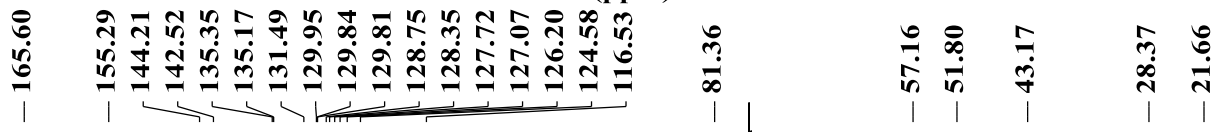

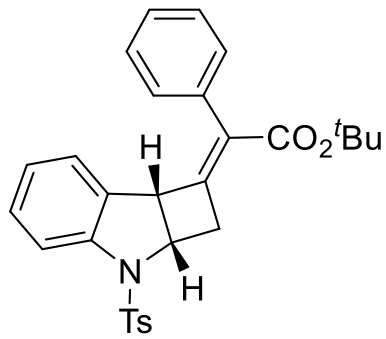

$(E)-3 a d$

$\left(125 \mathrm{MHz}\right.$; in $\mathrm{CDCl}_{3}$ )

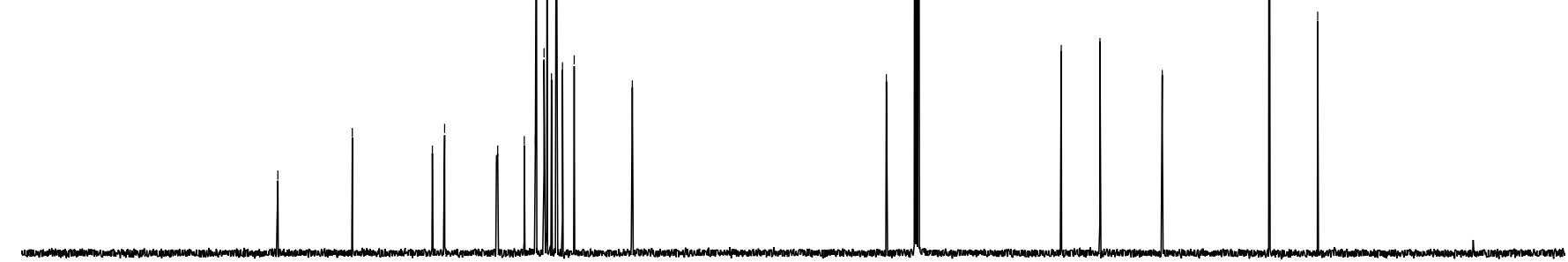




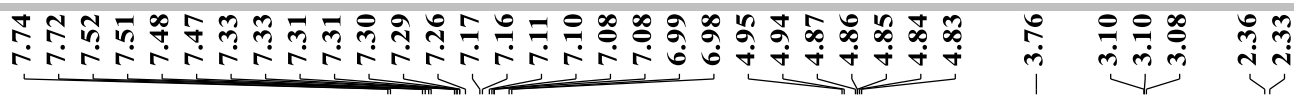

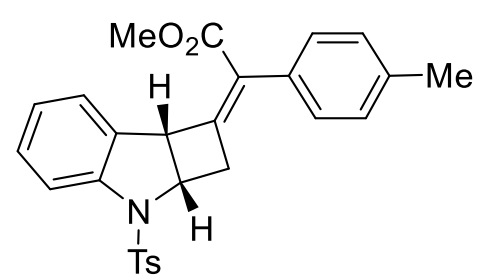

(Z)-3ae

$\left(500 \mathrm{MHz}\right.$; in $\mathrm{CDCl}_{3}$ )

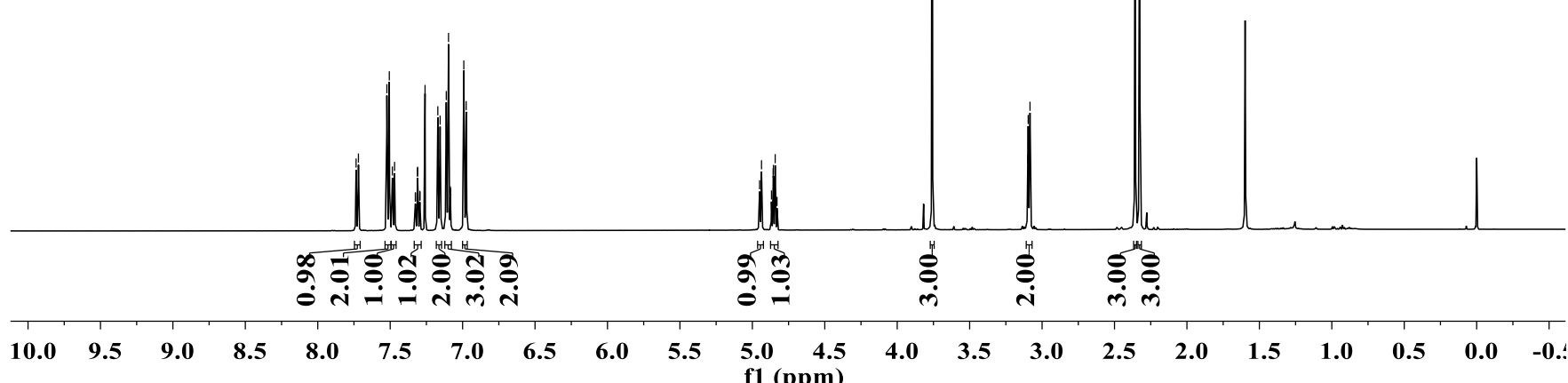

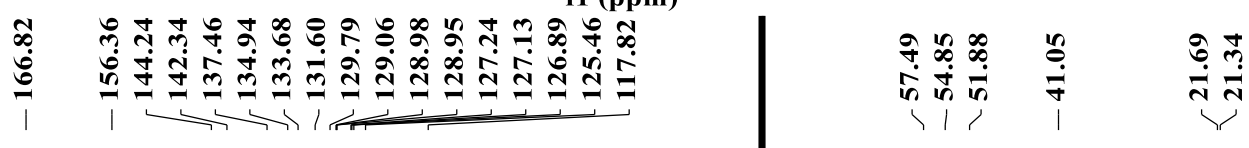

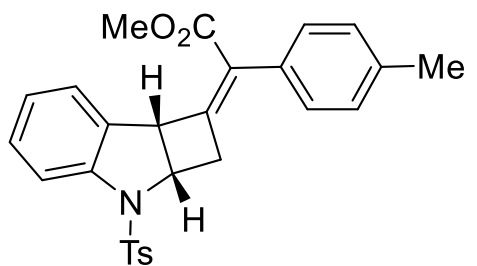

(Z)-3ae

(125 MHz; in $\mathrm{CDCl}_{3}$ ) 


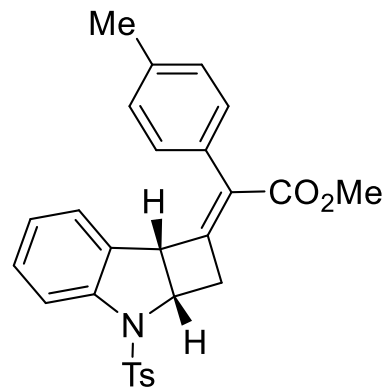

$(E)$-3ae

$\left(500 \mathrm{MHz}\right.$; in $\left.\mathrm{CDCl}_{3}\right)$

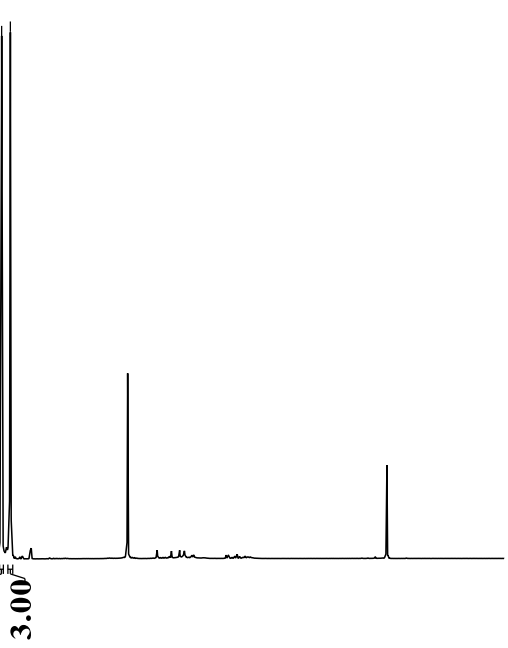

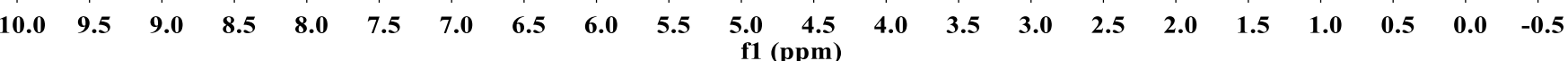

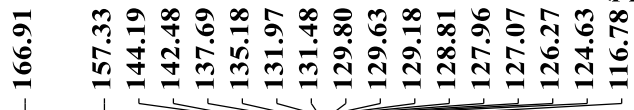

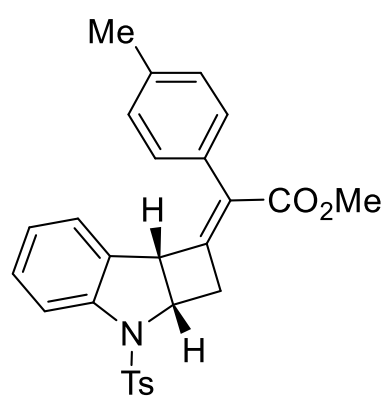

$(E)-3 a e$

$\left(125 \mathrm{MHz}\right.$; in $\left.\mathrm{CDCl}_{3}\right)$
تُ

î̉




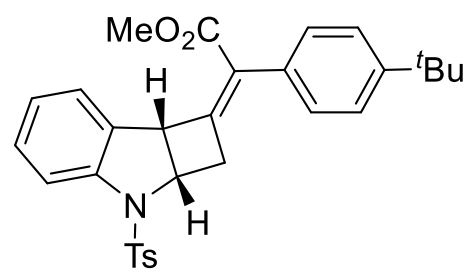

(Z)-3af

$\left(500 \mathrm{MHz}\right.$; in $\mathrm{CDCl}_{3}$ )

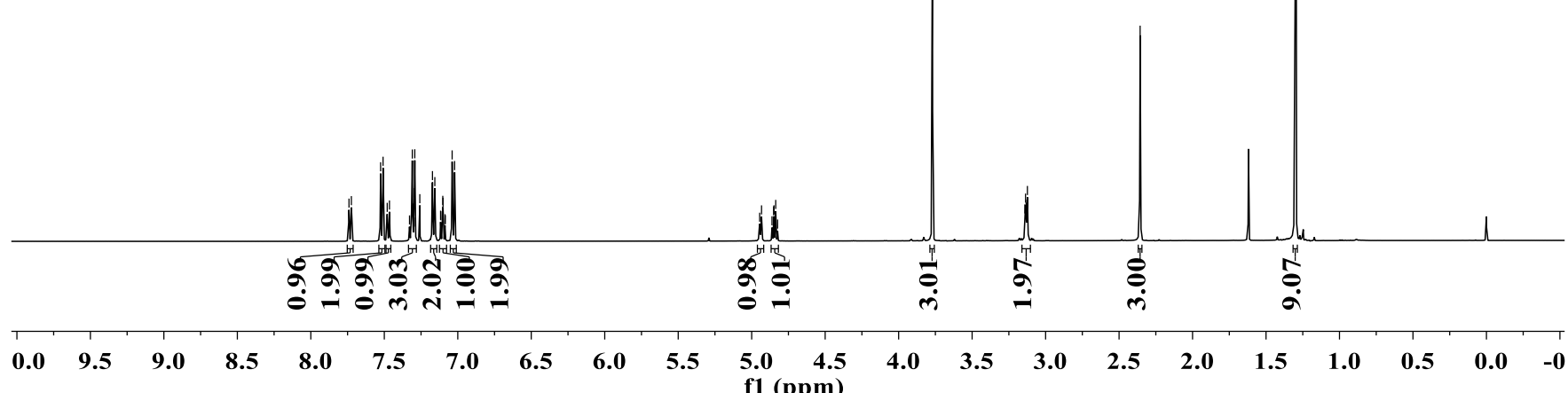

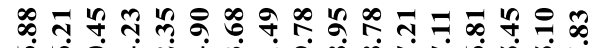

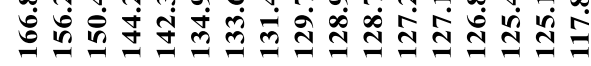

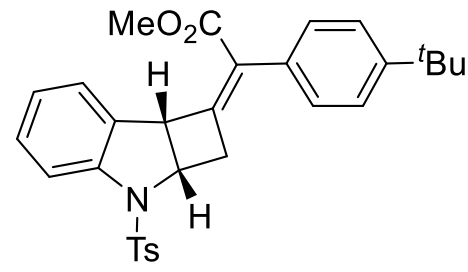

(Z)-3af

$\left(125 \mathrm{MHz}\right.$; in $\left.\mathrm{CDCl}_{3}\right)$

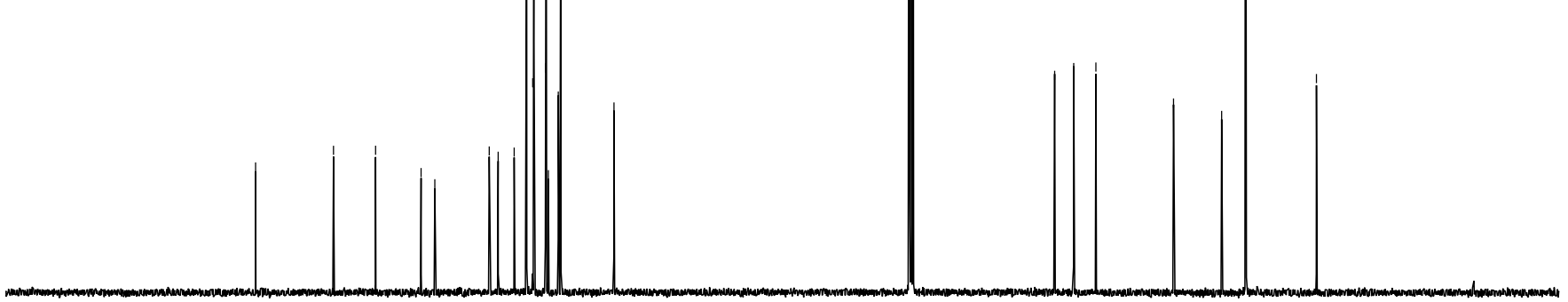




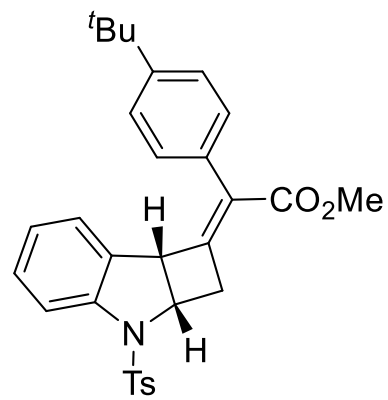

(E)-3af

$\left(500 \mathrm{MHz} ;\right.$ in $\mathrm{CDCl}_{3}$ )

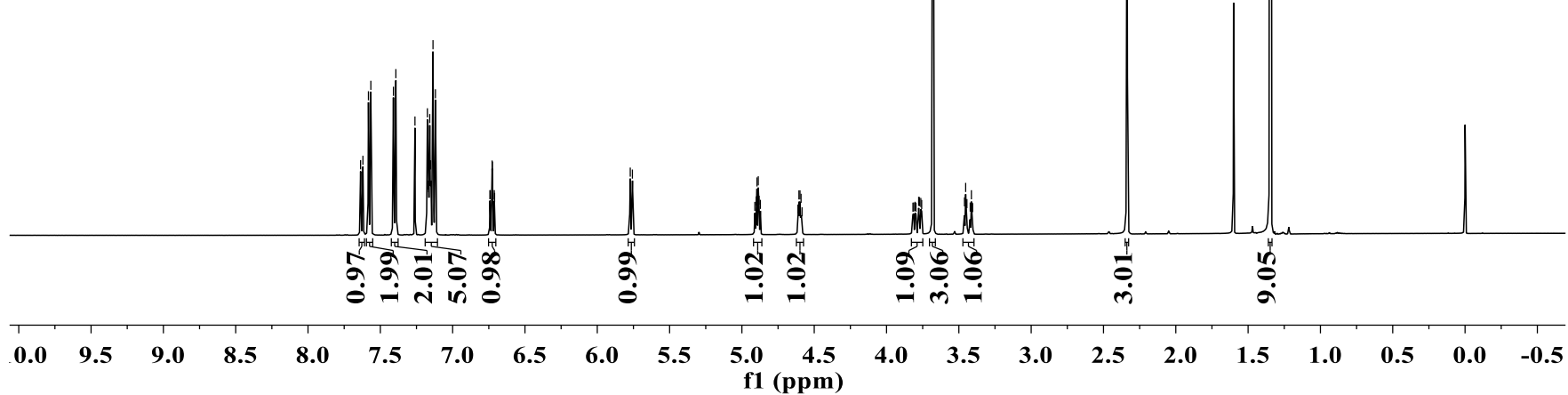

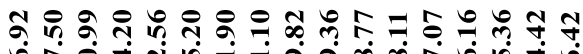

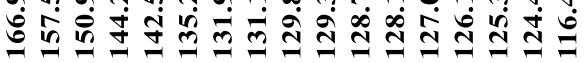

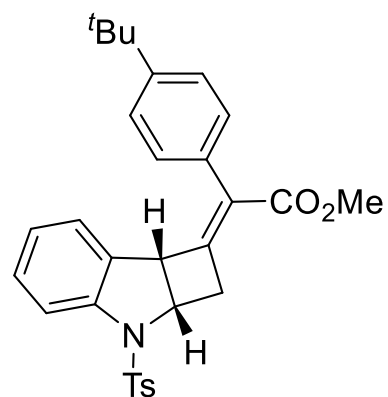

(E)-3af

$\left(125 \mathrm{MHz}\right.$; in $\mathrm{CDCl}_{3}$ ) 


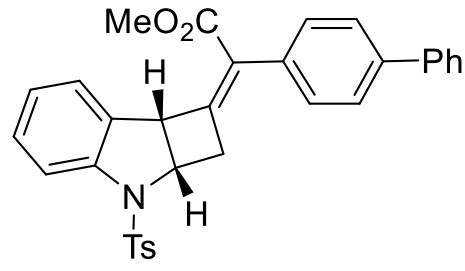

$$
\text { (Z)-3ag }
$$

(500 MHz; in $\mathrm{CDCl}_{3}$ )

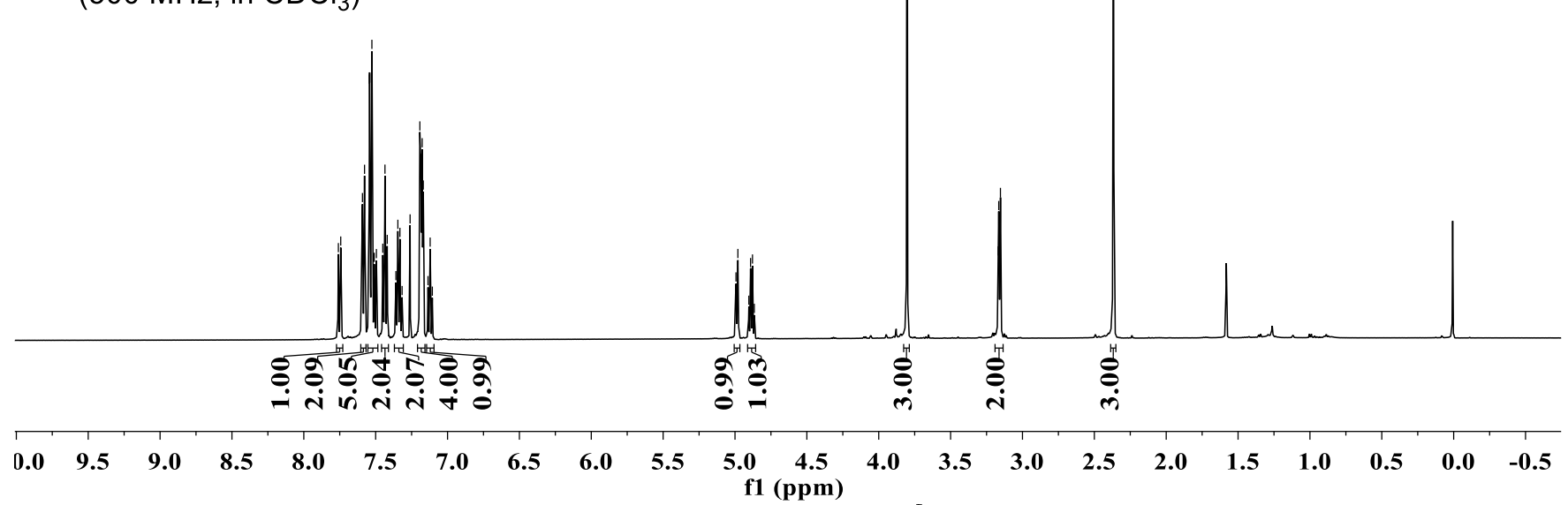

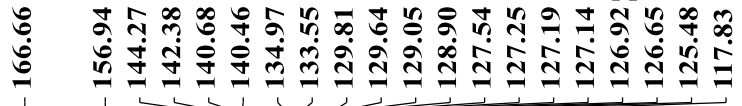

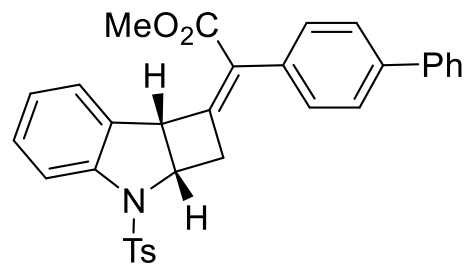

(Z)-3ag

$\left(125 \mathrm{MHz}\right.$; in $\mathrm{CDCl}_{3}$ )

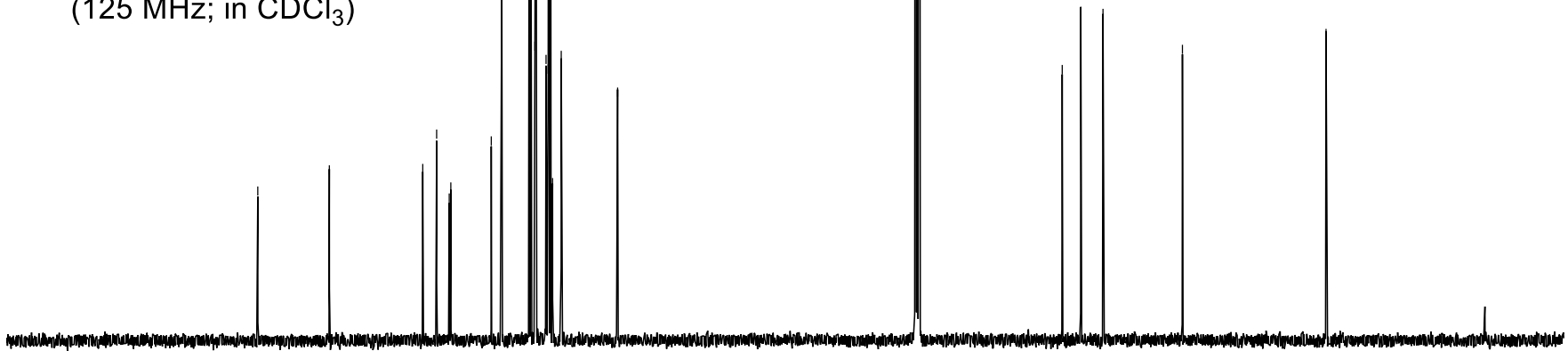




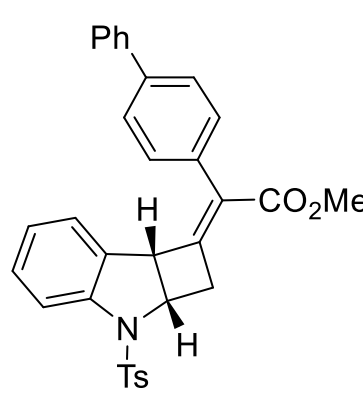

$(E)-3 a g$

$\left(500 \mathrm{MHz}\right.$; in $\mathrm{CDCl}_{3}$ )
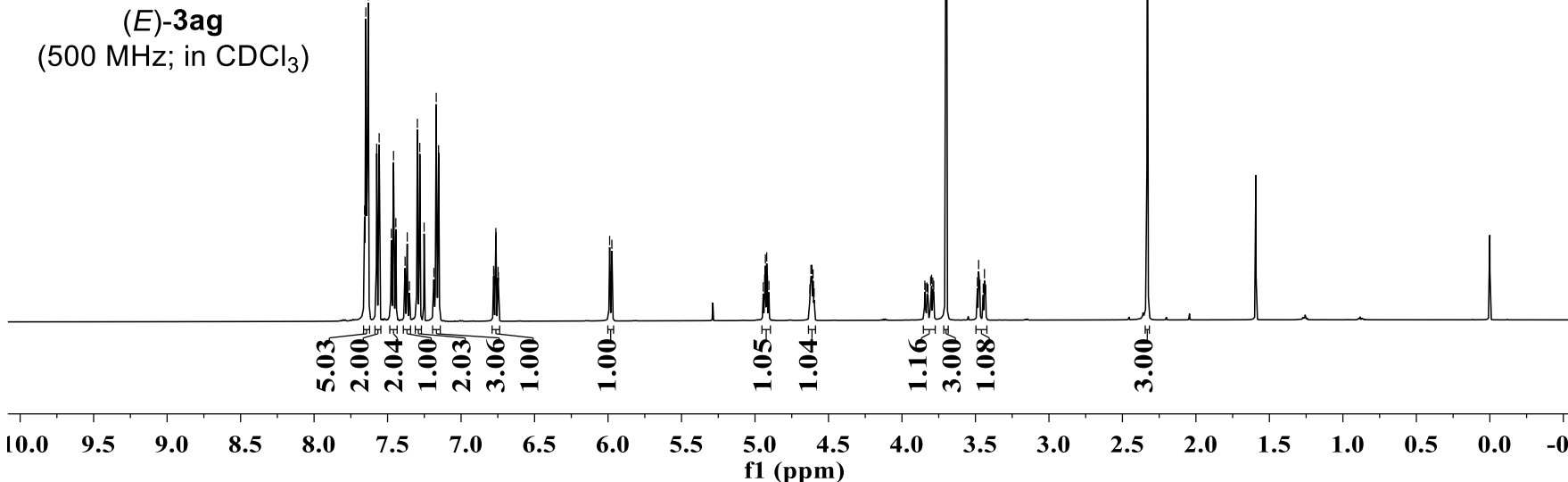
ำ 훙

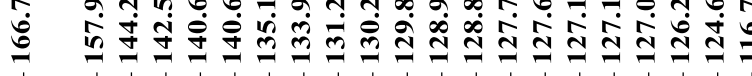<smiles>CC(=O)C(=C1C[C@@H]2[C@H]1c1ccccc1N2C)c1ccc(-c2ccccc2)cc1</smiles>

(E)-3ag

(125 MHz; in $\mathrm{CDCl}_{3}$ ) 


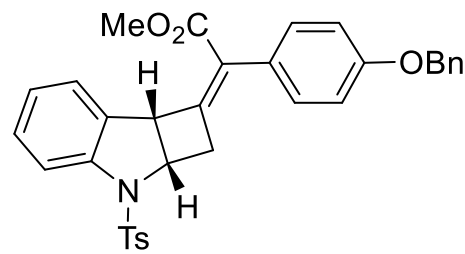

(Z)-3ah

(500 $\mathrm{MHz}$; in $\mathrm{CDCl}_{3}$ )

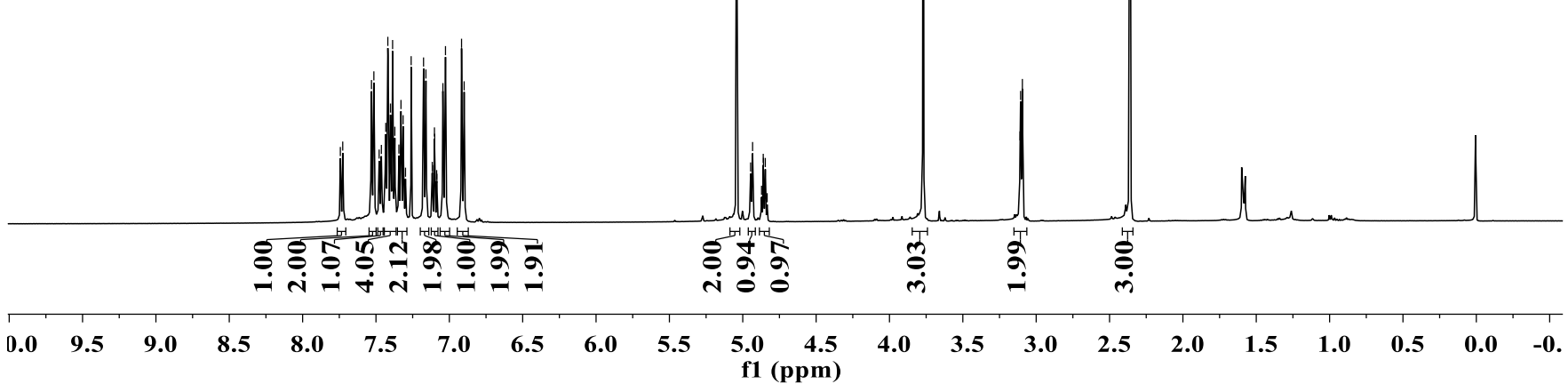

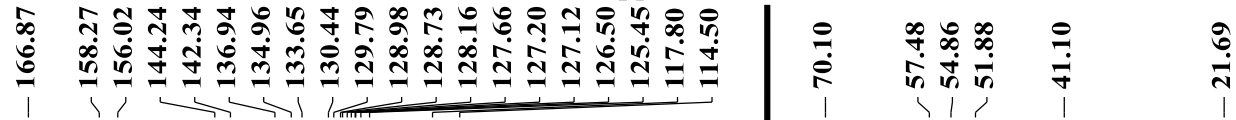

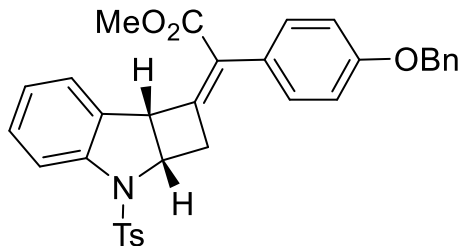

(Z)-3ah

$\left(125 \mathrm{MHz}\right.$; in $\mathrm{CDCl}_{3}$ ) 


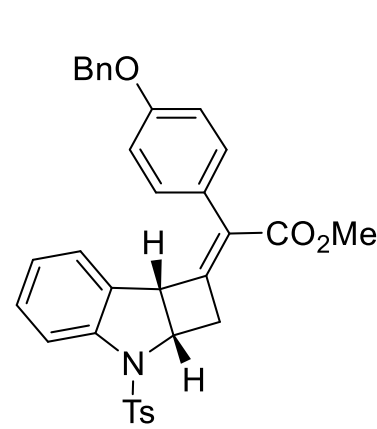

(E)-3ah

$\left(500 \mathrm{MHz} ;\right.$ in $\left.\mathrm{CDCl}_{3}\right)$

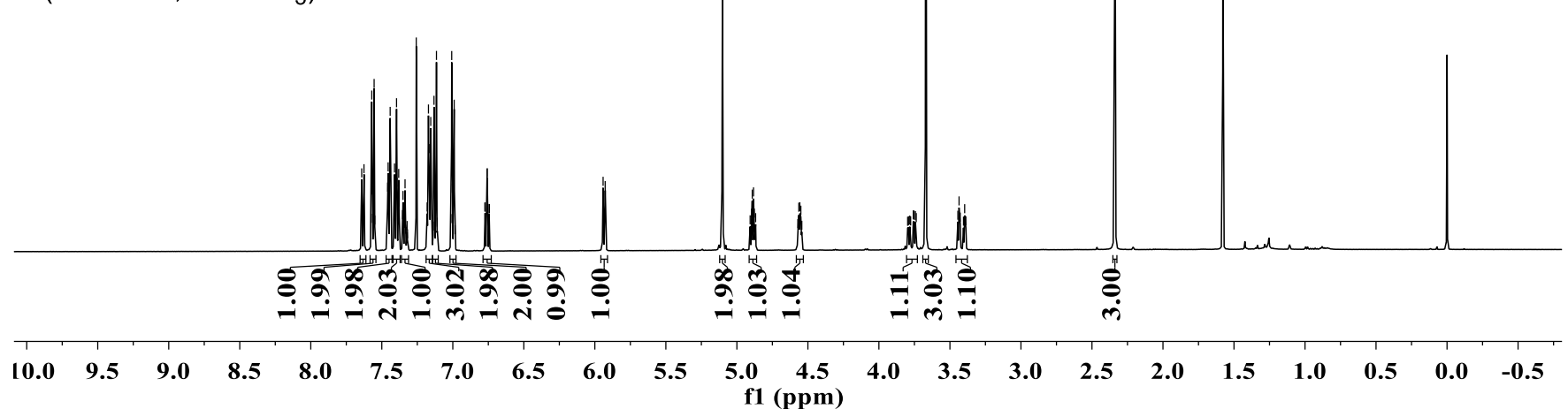

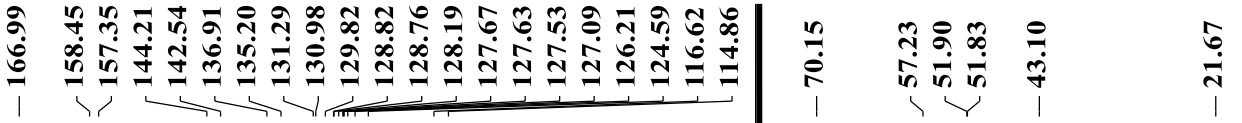

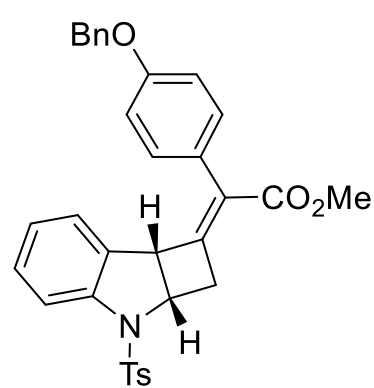

(E)-3ah

$\left(125 \mathrm{MHz}\right.$; in $\left.\mathrm{CDCl}_{3}\right)$ 


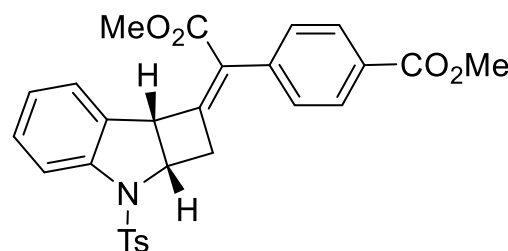

(Z)-3ai

$\left(500 \mathrm{MHz}\right.$; in $\left.\mathrm{CDCl}_{3}\right)$

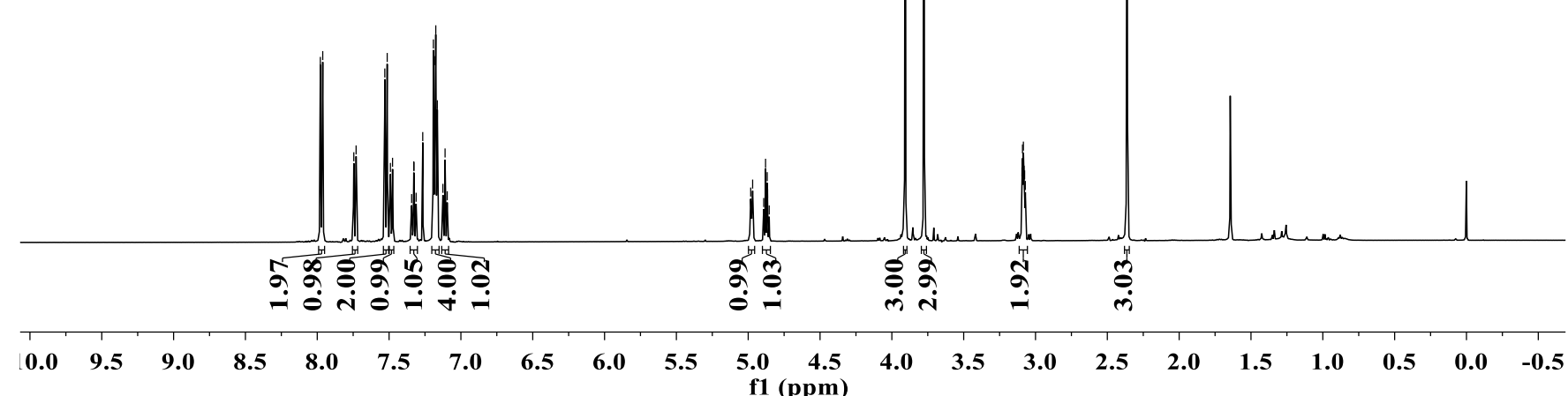

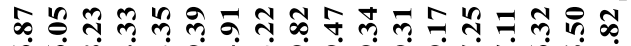

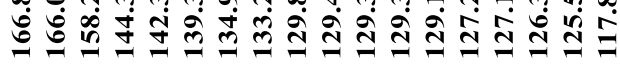

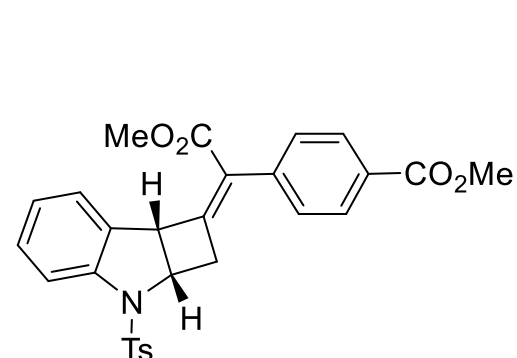

(Z)-3ai

$\left(125 \mathrm{MHz}\right.$; in $\left.\mathrm{CDCl}_{3}\right)$ 


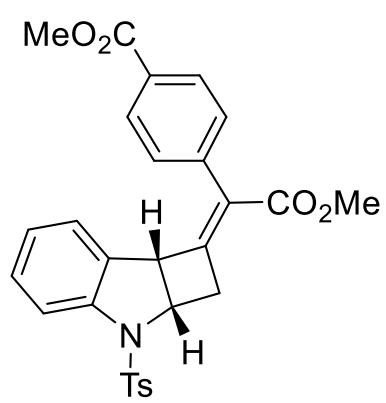

$(E)-3$ ai

$\left(500 \mathrm{MHz}\right.$; in $\left.\mathrm{CDCl}_{3}\right)$

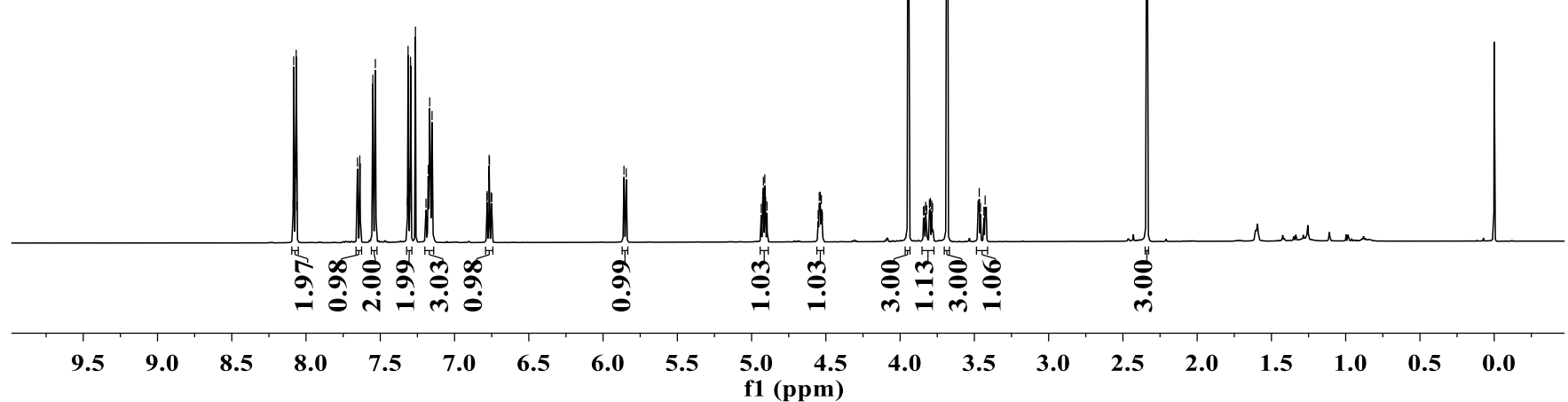

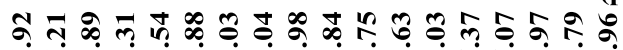

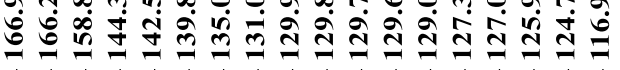

సุ ले ڤั

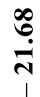

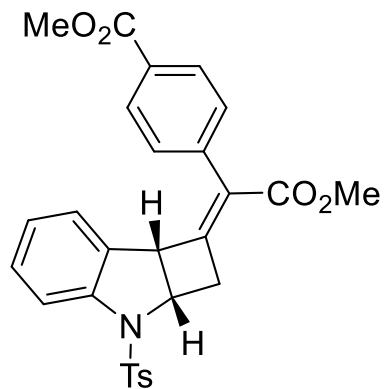

$(E)-3 \mathbf{a i}$

$\left(125 \mathrm{MHz}\right.$; in $\left.\mathrm{CDCl}_{3}\right)$

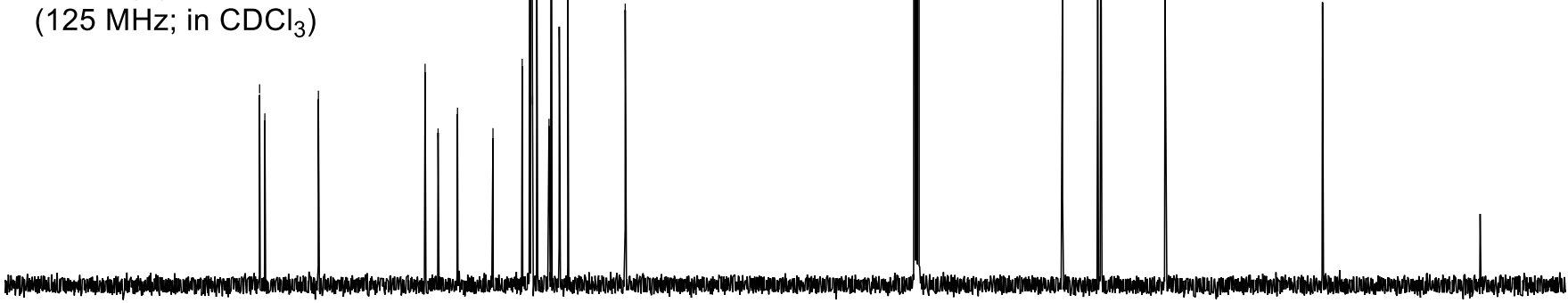




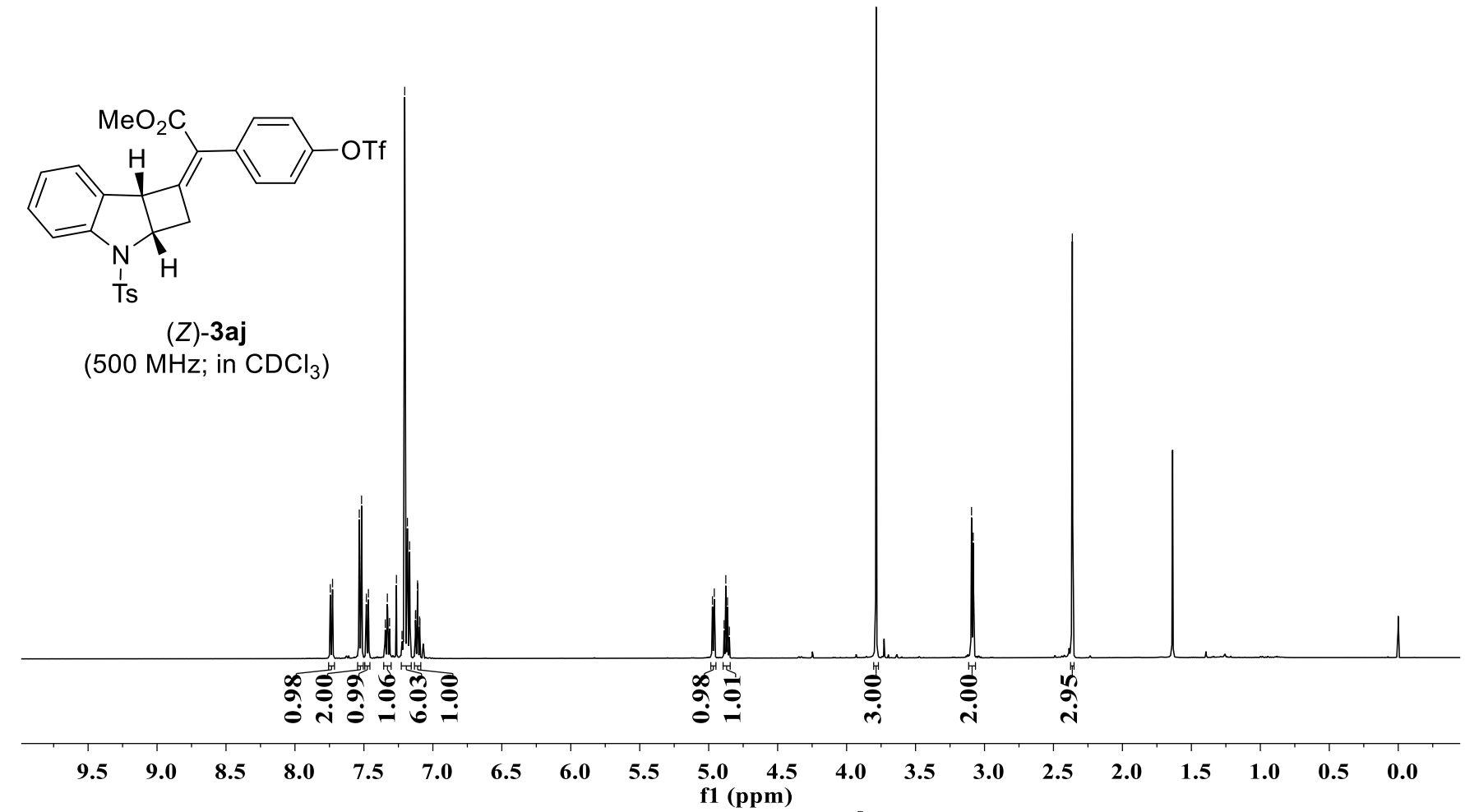
के कि

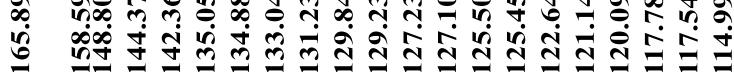
1。ำ

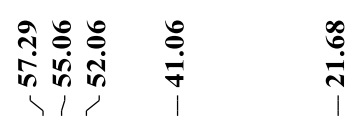

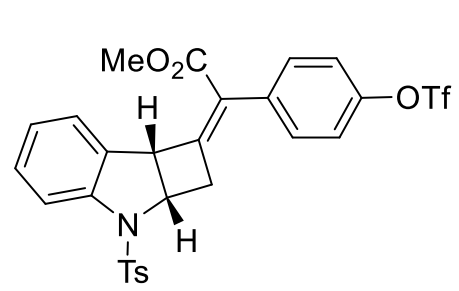

(Z)-3aj

$\left(125 \mathrm{MHz}\right.$; in $\mathrm{CDCl}_{3}$ )

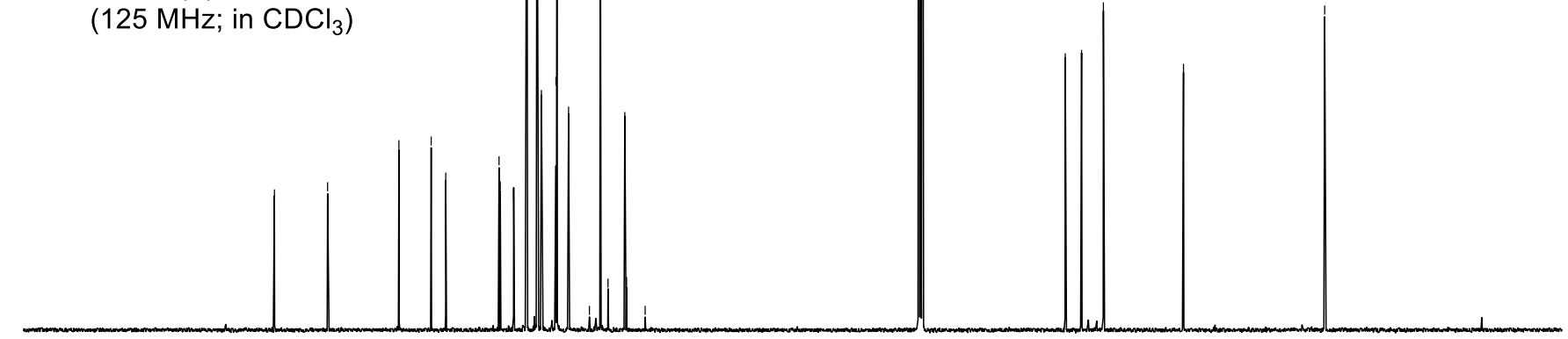




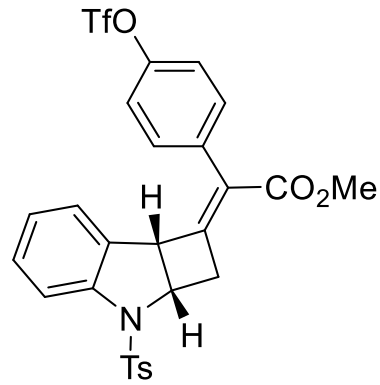

(E)-3aj

$\left(500 \mathrm{MHz}\right.$; in $\mathrm{CDCl}_{3}$ )

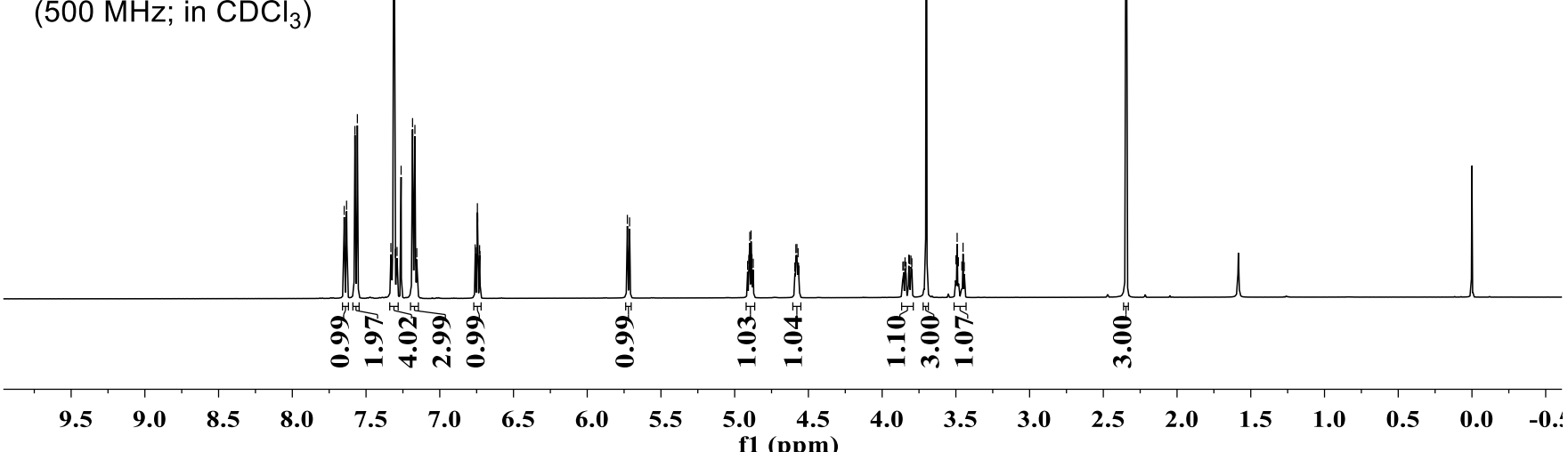

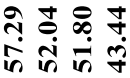

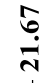

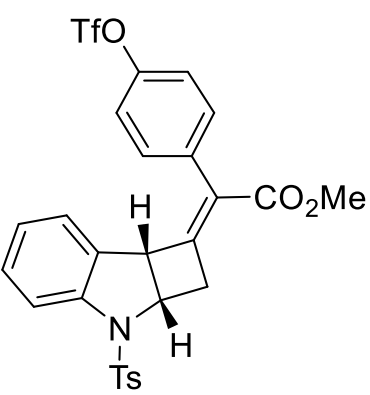

(E)-3aj

$\left(125 \mathrm{MHz}\right.$; in $\mathrm{CDCl}_{3}$ )
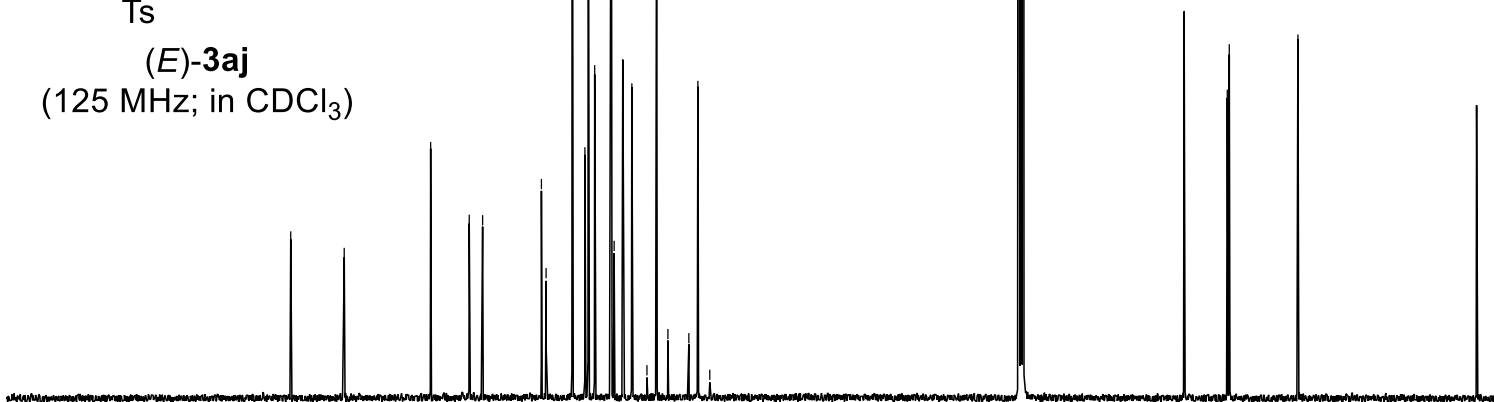

$\begin{array}{lllllllllll}190 & 180 & 170 & 160 & 150 & 140 & 130 & 120 & 110 & 100 & 90\end{array}$ 


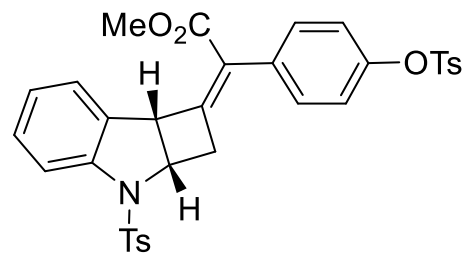

(Z)-3ak

$\left(500 \mathrm{MHz}\right.$; in $\left.\mathrm{CDCl}_{3}\right)$

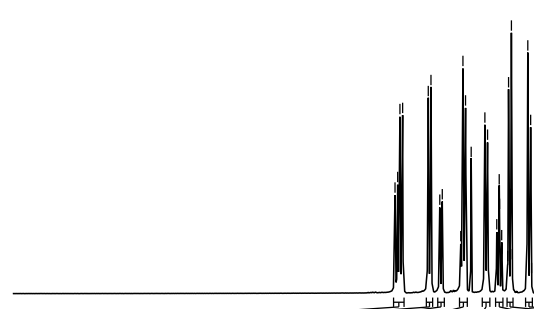

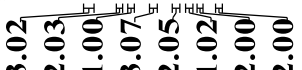

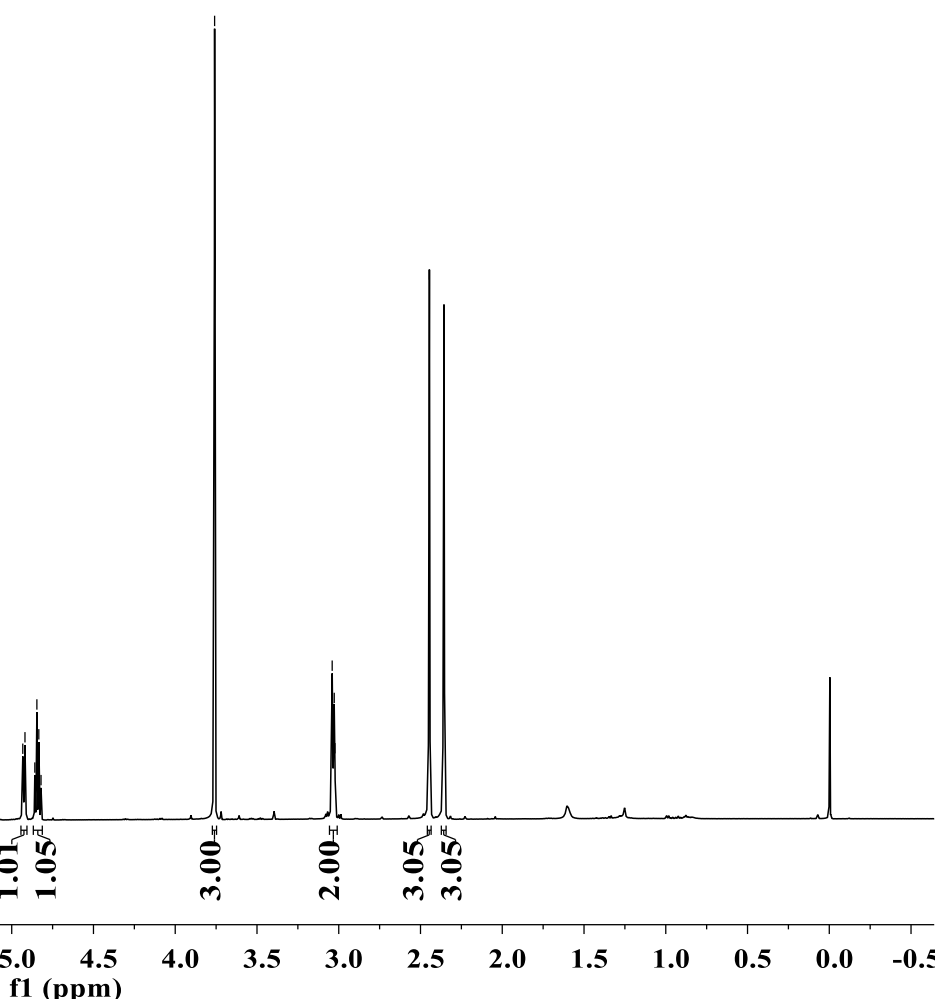

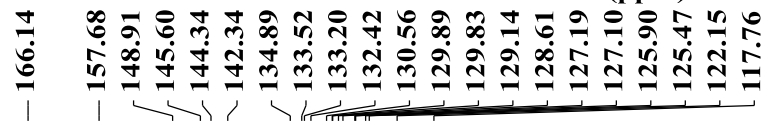

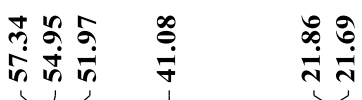

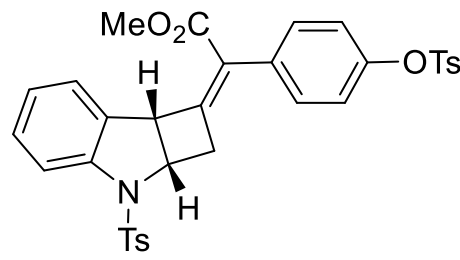

(Z)-3ak

$\left(125 \mathrm{MHz}\right.$; in $\mathrm{CDCl}_{3}$ )

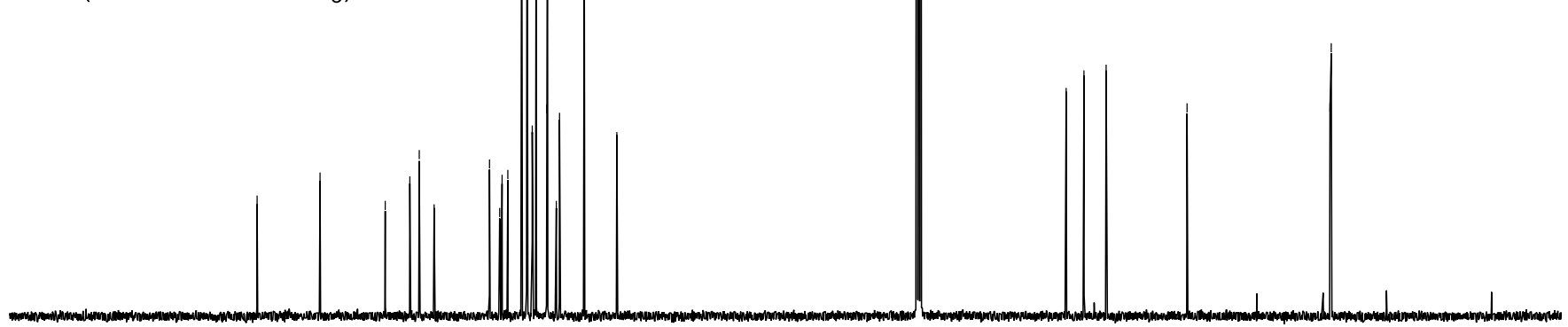


<smiles>[3H]N1c2ccccc2[C@H]2/C(=C(\C(=O)OC)c3ccc(OC4CCCCC4)cc3)C[C@@H]21</smiles>

(E)-3ak

(500 MHz; in $\mathrm{CDCl}_{3}$ )

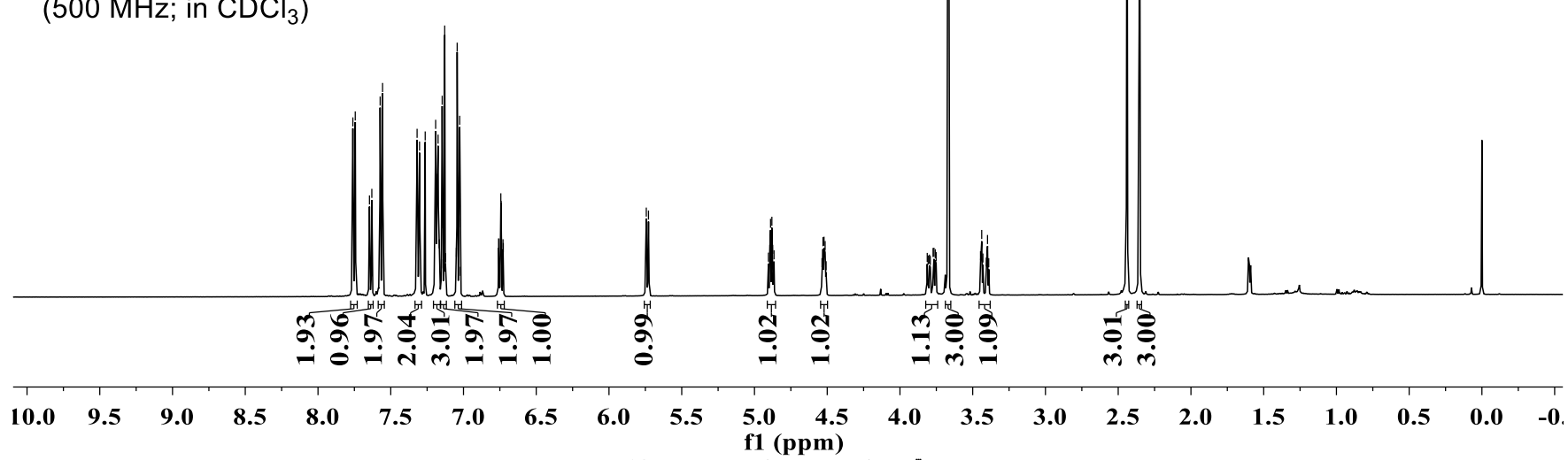

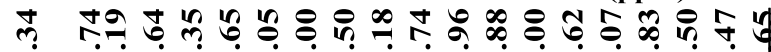

苟

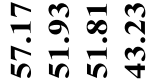

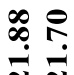

in

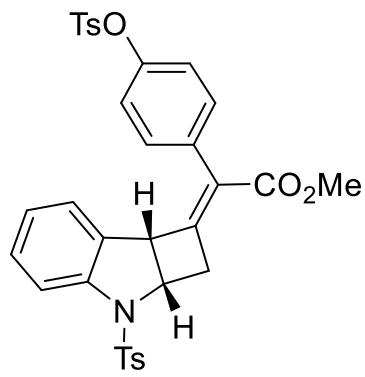

(E)-3ak

(125 MHz; in $\mathrm{CDCl}_{3}$ )

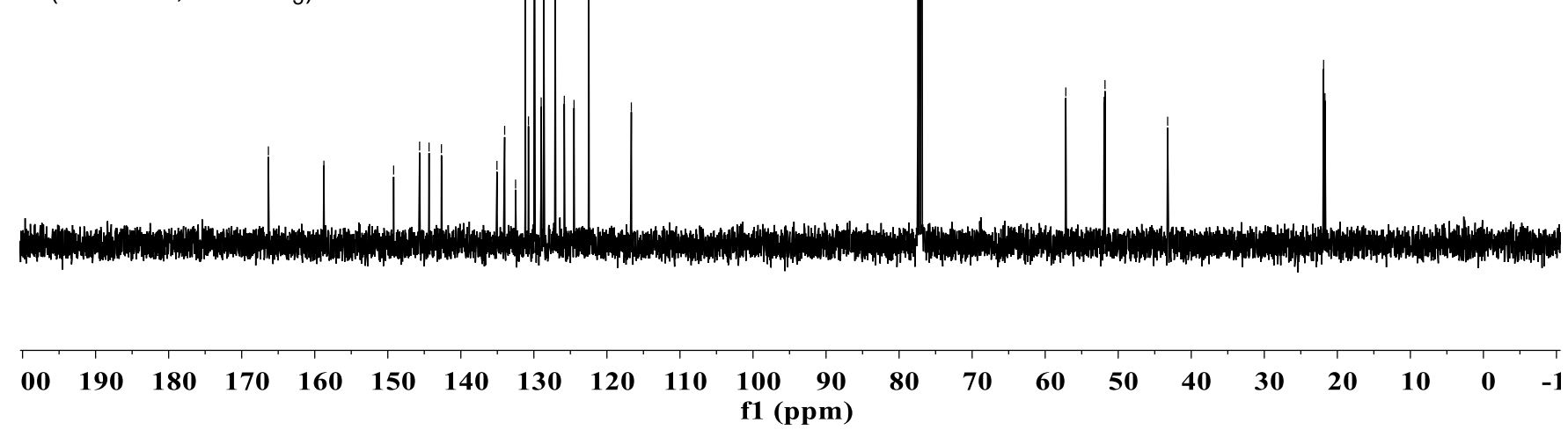




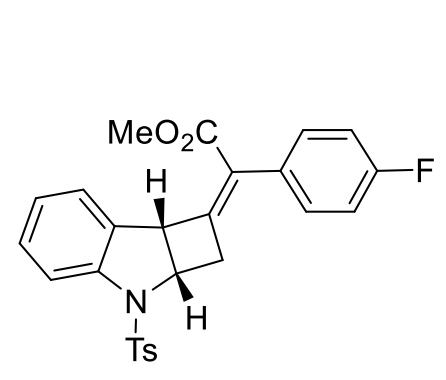

(Z)-3al

(500 MHz; in $\mathrm{CDCl}_{3}$ )

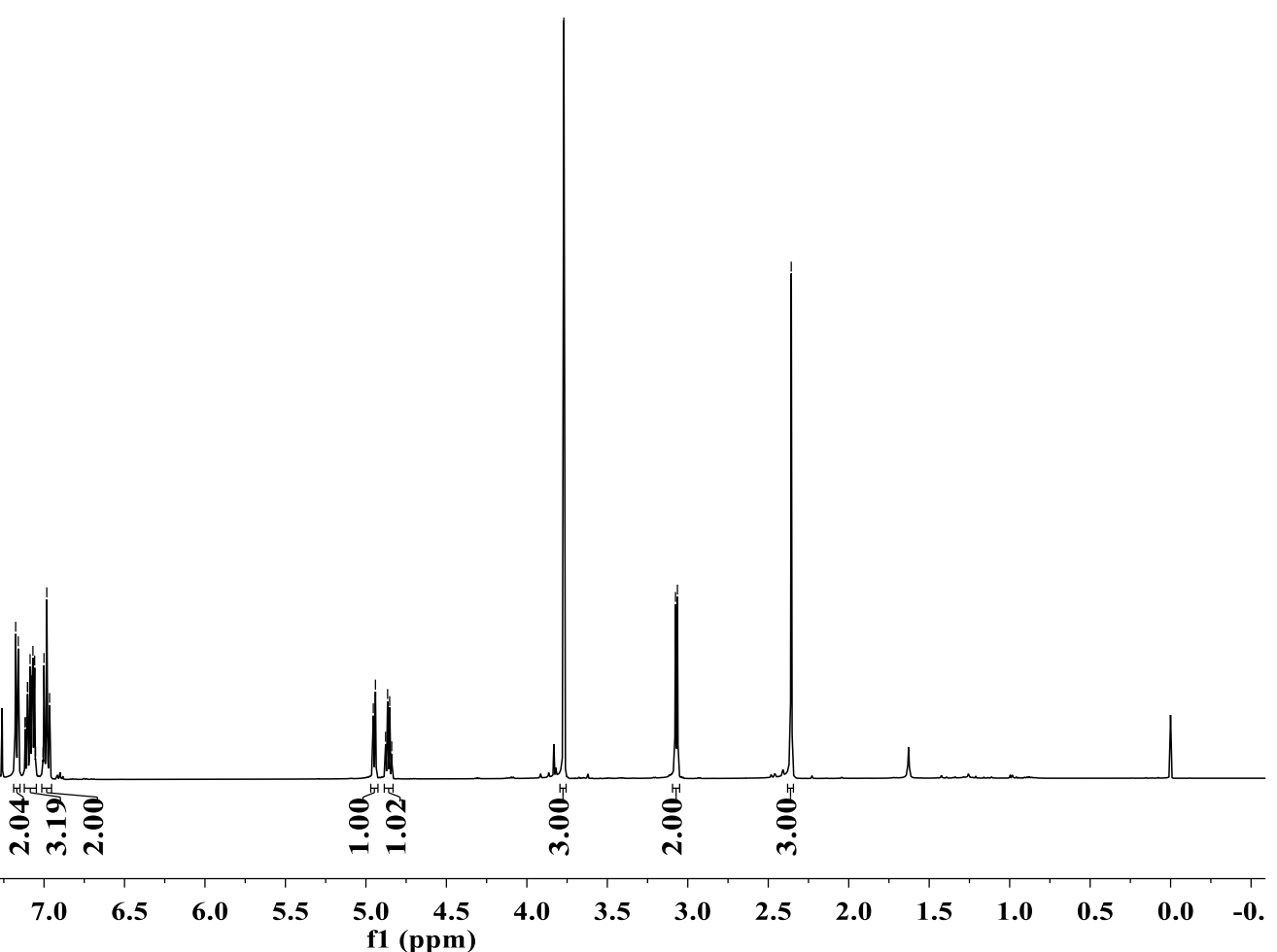

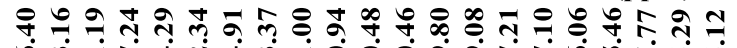

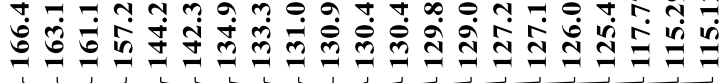

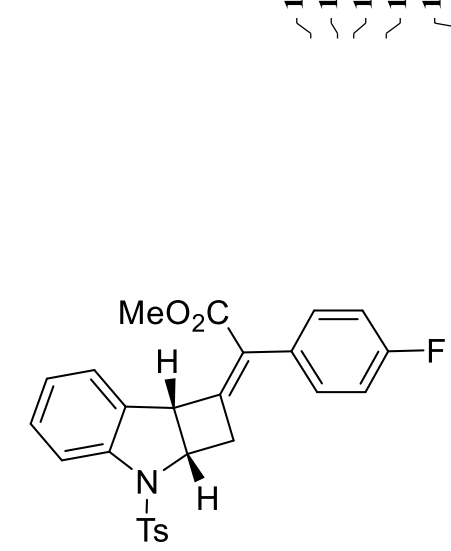

(Z)-3al

(125 MHz; in $\mathrm{CDCl}_{3}$ )

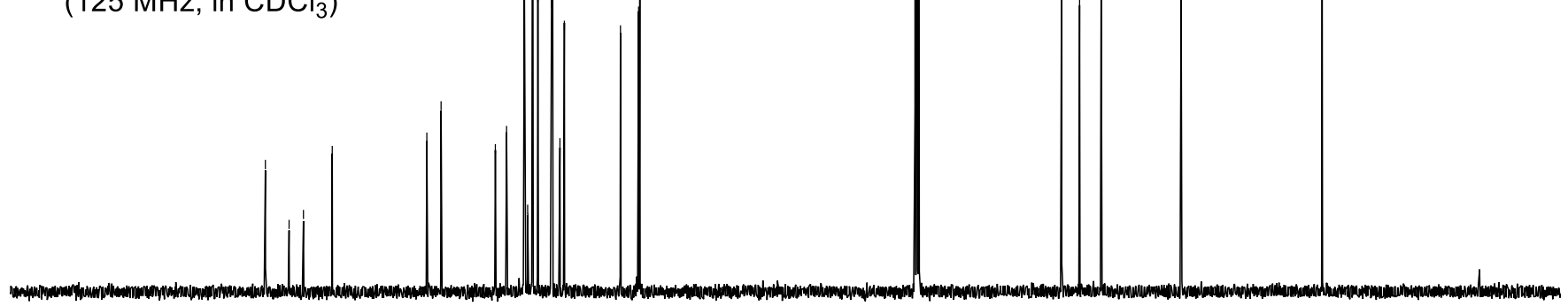


<smiles>COC(=O)C1=CC2C[C@H]1c1ccccc1N2[13CH3]</smiles>

(E)-3al

$\left(500 \mathrm{MHz}\right.$; in $\mathrm{CDCl}_{3}$ )

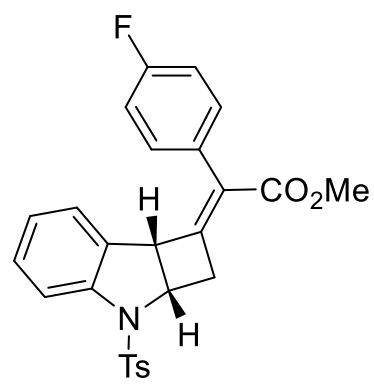

(E)-3al

(125 MHz; in $\mathrm{CDCl}_{3}$ )

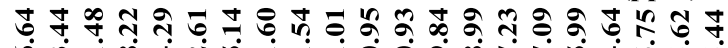

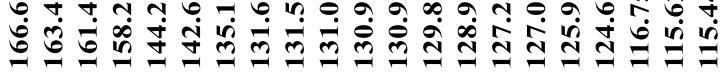

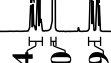

ํ.

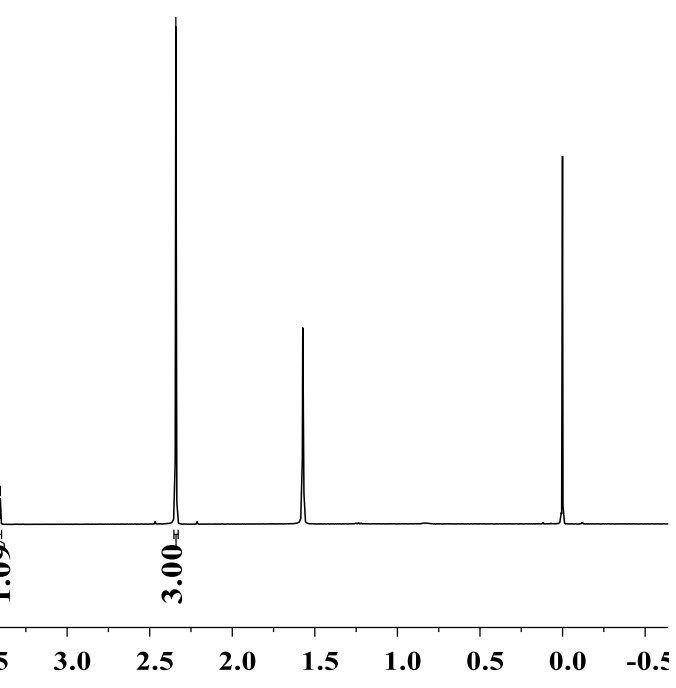

กิ่ 


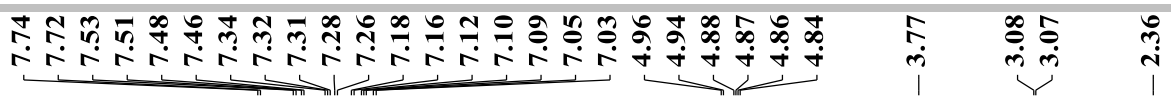

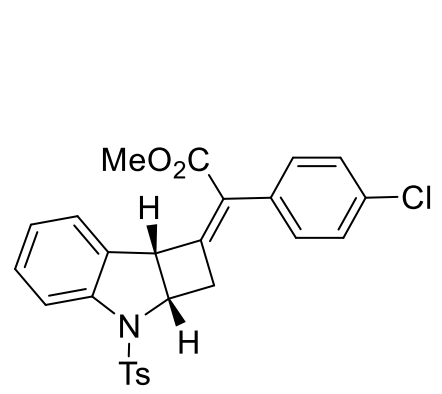

(Z)-3am

$\left(500 \mathrm{MHz}\right.$; in $\mathrm{CDCl}_{3}$ )

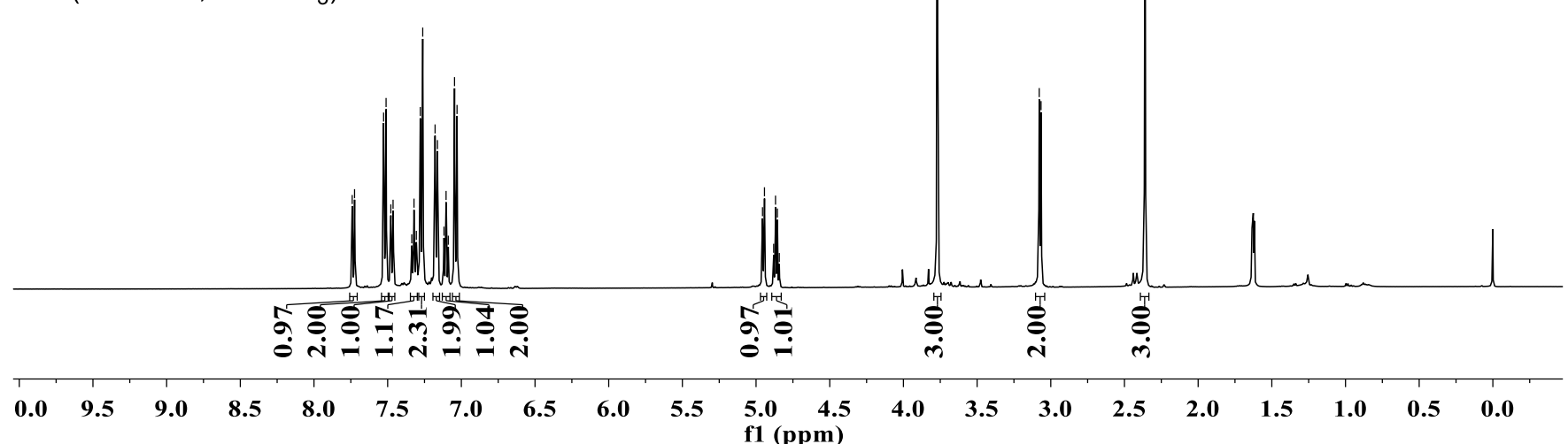

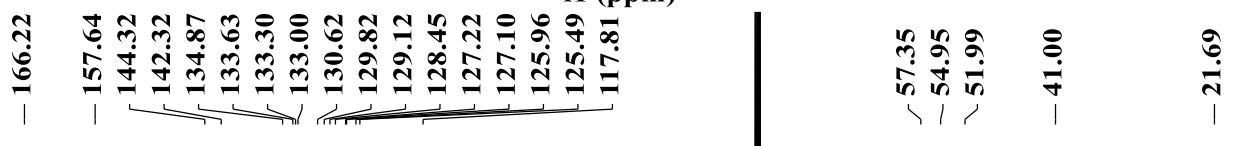

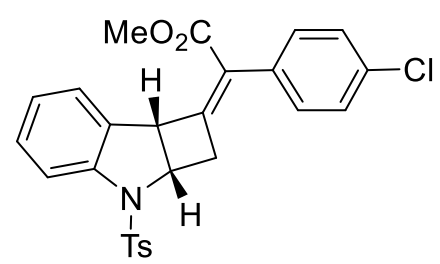

(Z)-3am

$\left(125 \mathrm{MHz}\right.$; in $\left.\mathrm{CDCl}_{3}\right)$

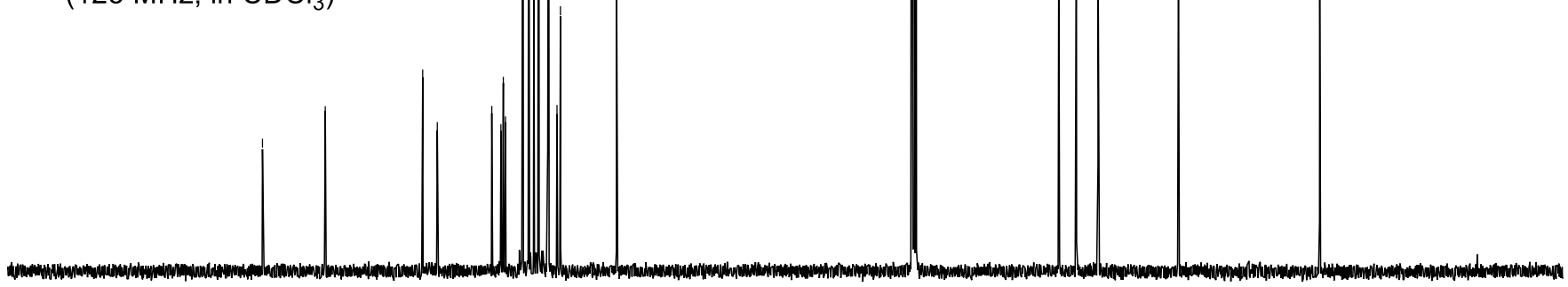




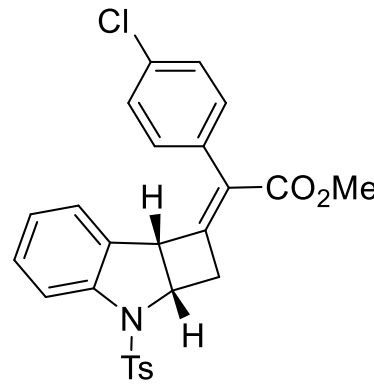

(E)-3am

$\left(500 \mathrm{MHz} ;\right.$ in $\left.\mathrm{CDCl}_{3}\right)$

$$
\left(500 \mathrm{MHz}^{2} \mathrm{in} \mathrm{CDCl}_{3}\right)
$$

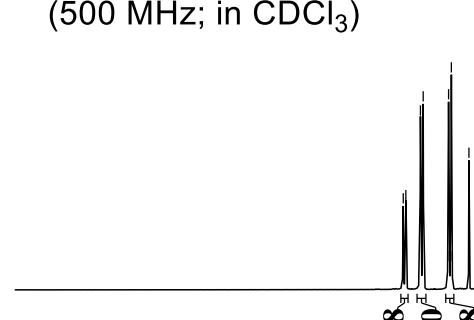

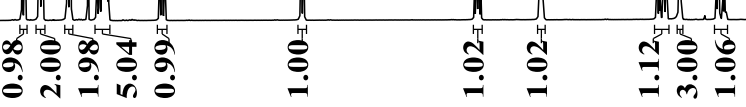

$\begin{array}{lllllllllll}0.0 & 9.5 & 9.0 & 8.5 & 8.0 & 7.5 & 7.0 & 6.5 & 6.0 & 5.5 & \begin{array}{rl}5.0 & 4.5 \\ \text { f1 (ppm) }\end{array}\end{array}$

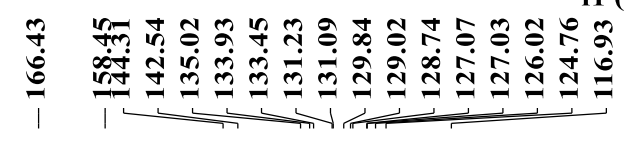

f1 (ppm)

4.0

in

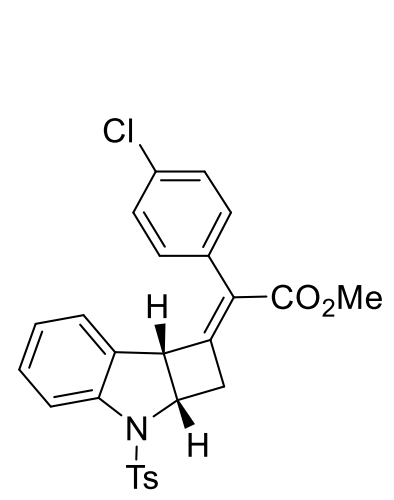

(E)-3am

$\left(125 \mathrm{MHz}\right.$; in $\mathrm{CDCl}_{3}$ )

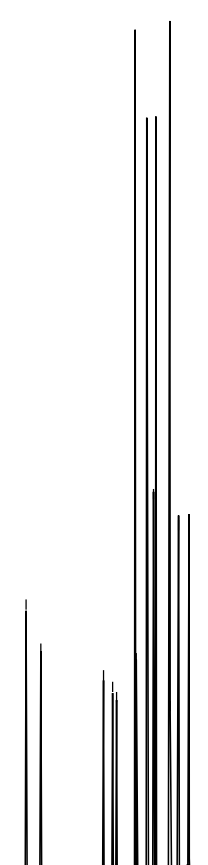


<smiles>[3H]N1c2ccccc2[C@@H]2[C@H]3/C(=C(\C(=O)OC)c4ccc(Br)cc4)C[C@@H]3[C@@H]21</smiles>

(Z)-3an

(500 MHz; in $\mathrm{CDCl}_{3}$ )

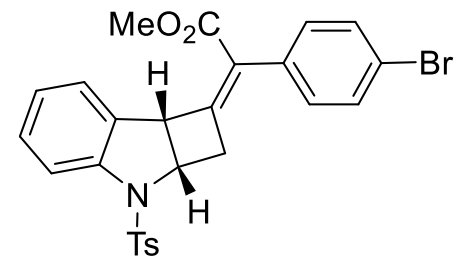

(Z)-3an

(125 MHz; in $\mathrm{CDCl}_{3}$ )

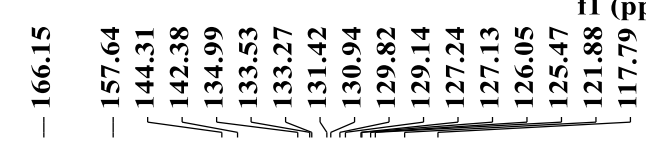

în 


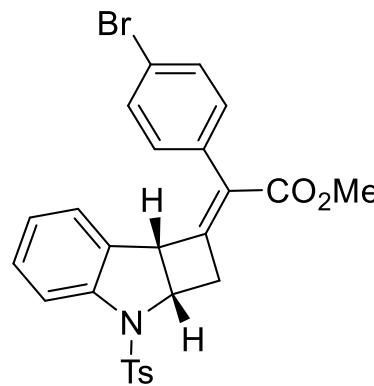

(E)-3an

$\left(500 \mathrm{MHz}\right.$; in $\mathrm{CDCl}_{3}$ )
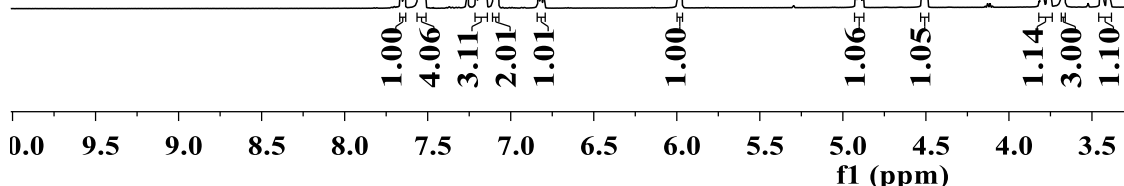

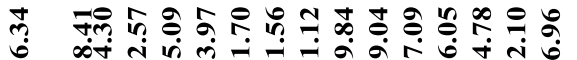

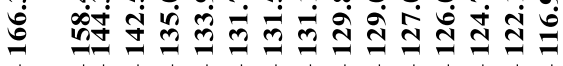

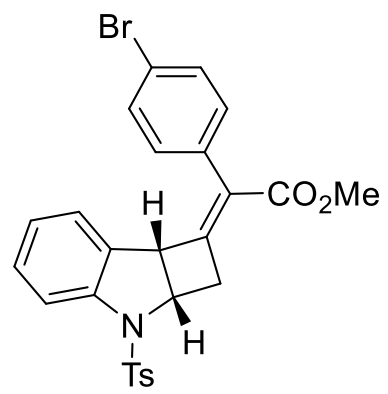

(E)-3an

$\left(125 \mathrm{MHz}\right.$; in $\left.\mathrm{CDCl}_{3}\right)$

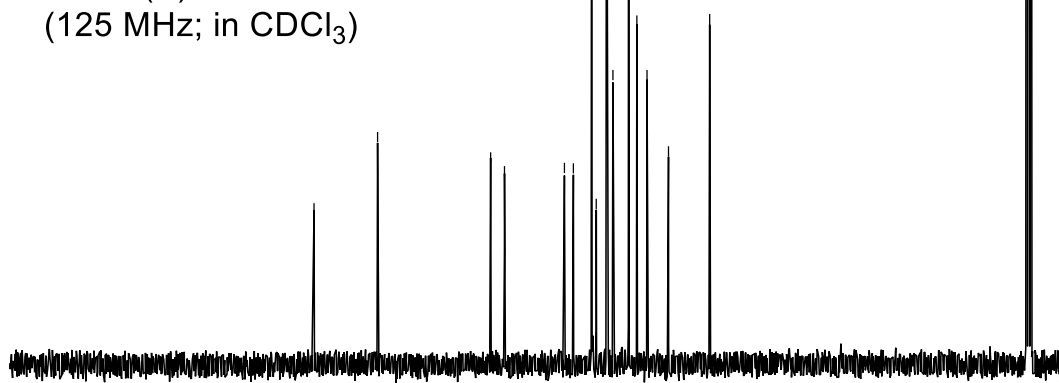


<smiles></smiles>

(Z)-3ao

$\left(500 \mathrm{MHz}\right.$; in $\mathrm{CDCl}_{3}$ )

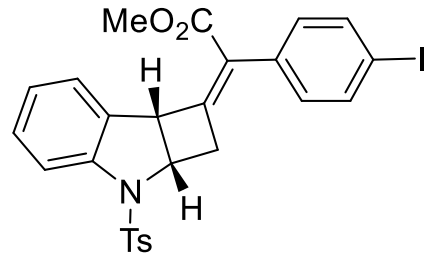

(Z)-3ao

$\left(125 \mathrm{MHz}\right.$; in $\mathrm{CDCl}_{3}$ )

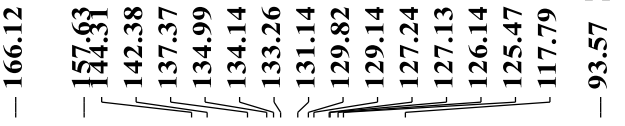

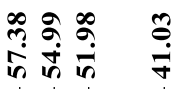

$\stackrel{\text { ำ }}{1}$ 


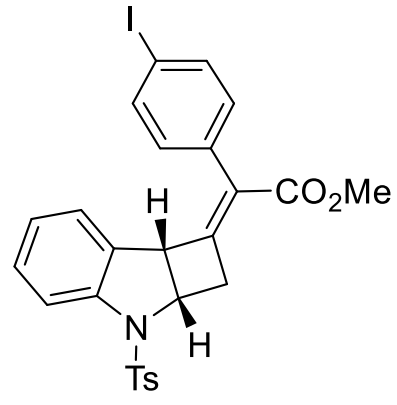

(E)-3ao

$\left(500 \mathrm{MHz}\right.$; in $\left.\mathrm{CDCl}_{3}\right)$

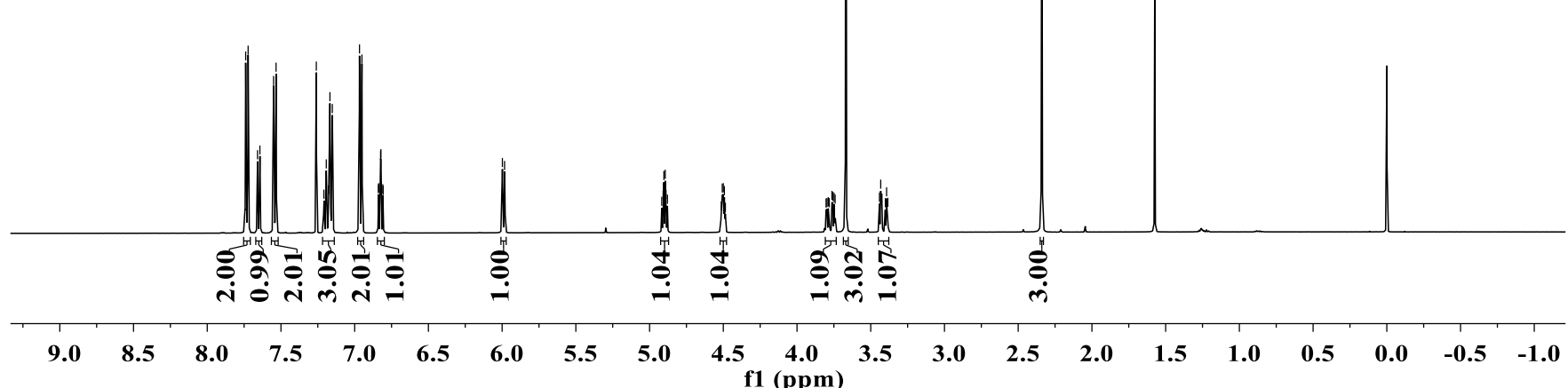

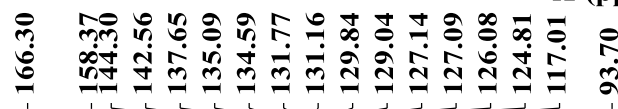

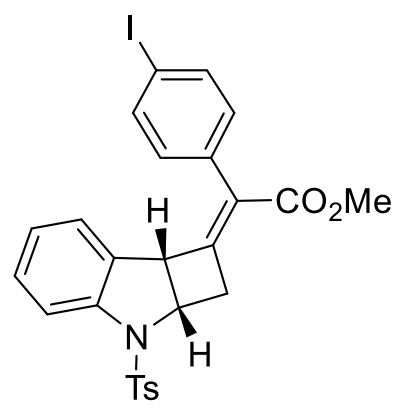

(E)-3ao

$\left(125 \mathrm{MHz}\right.$; in $\left.\mathrm{CDCl}_{3}\right)$

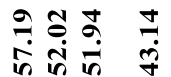

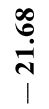




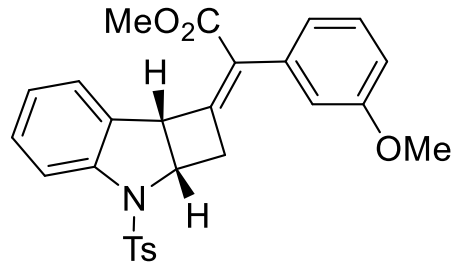

(Z)-3ap

$\left(500 \mathrm{MHz}\right.$; in $\mathrm{CDCl}_{3}$ )

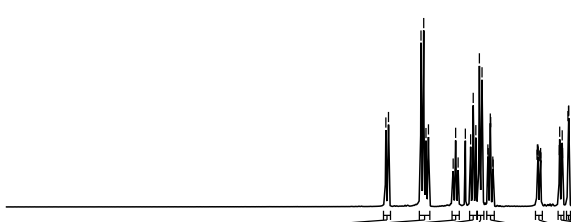

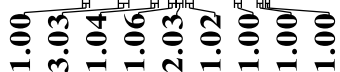

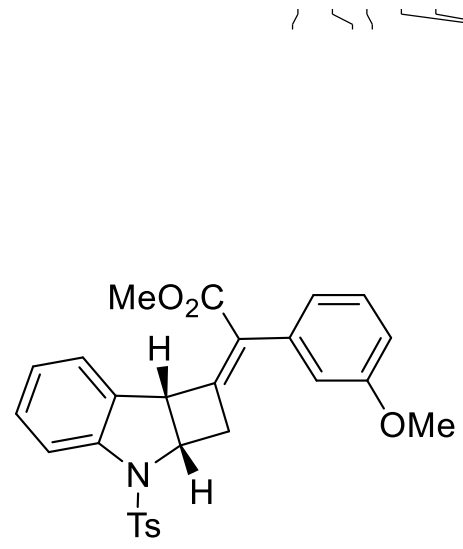

(Z)-3ap

$\left(125 \mathrm{MHz}\right.$; in $\mathrm{CDCl}_{3}$ )
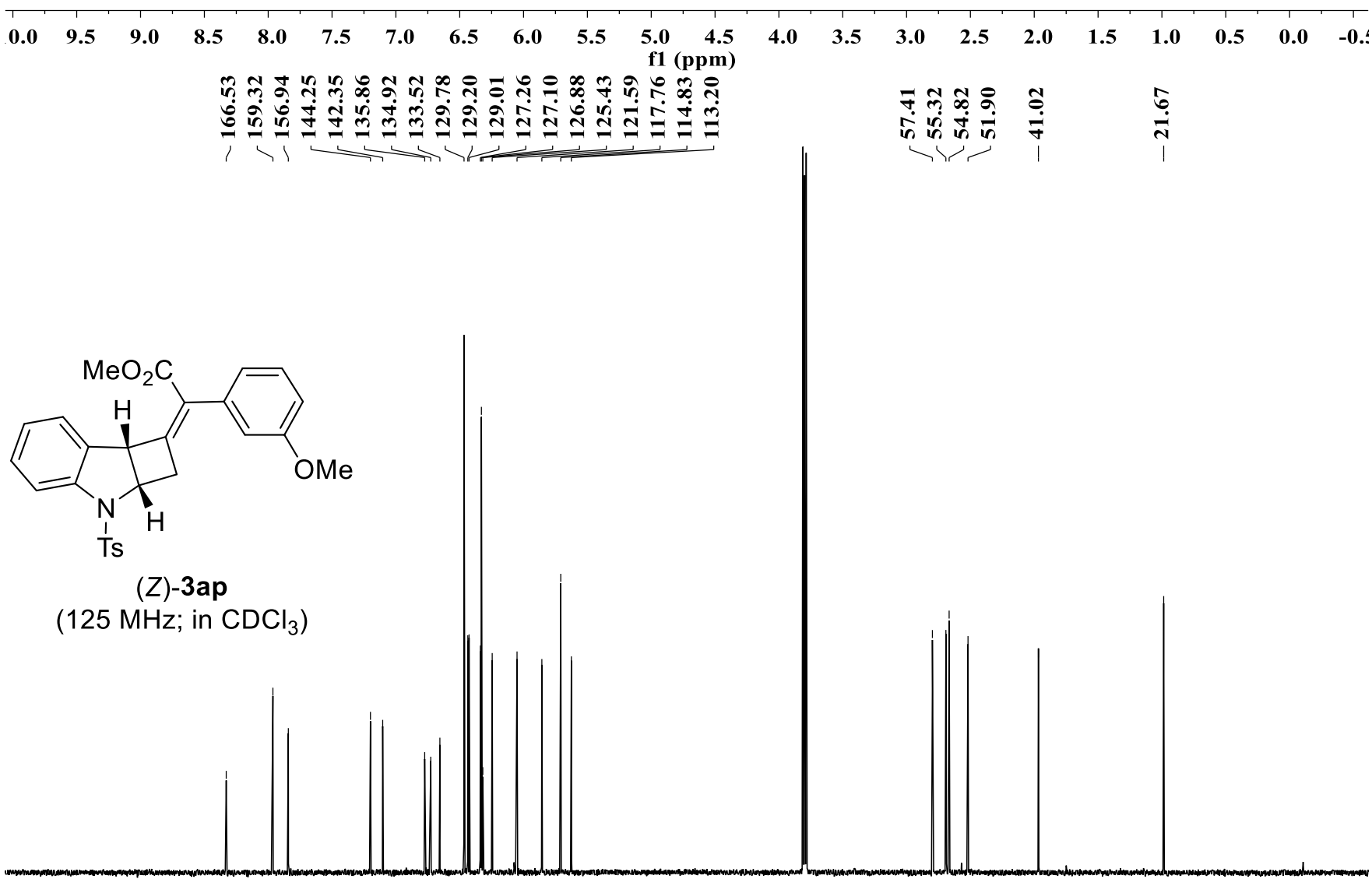

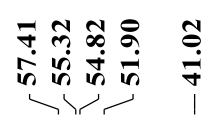

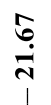


<smiles>COC(=O)/C(=C1\C[C@H]2[C@H]1c1ccccc1N2[As])c1cccc(OC)c1</smiles>

(E)-3ap

$\left(500 \mathrm{MHz} ;\right.$ in $\left.\mathrm{CDCl}_{3}\right)$

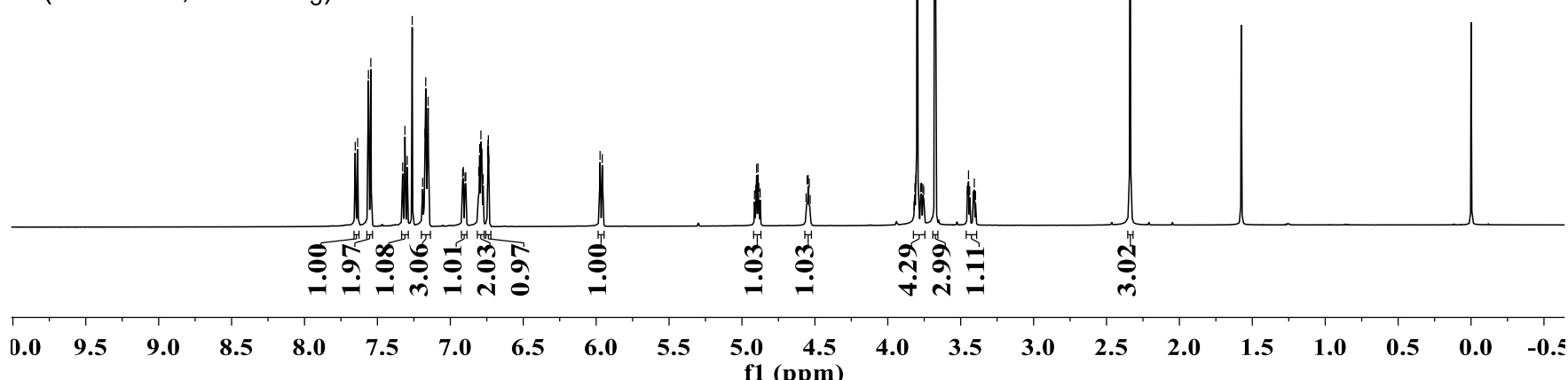

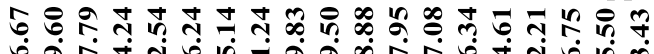

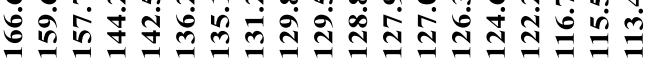<smiles>COC(=O)/C(=C1\C[C@H]2[C@H]1c1ccccc1N2[AsH])c1cccc(OC)c1</smiles>

(E)-3ap

(125 MHz; in $\mathrm{CDCl}_{3}$ )

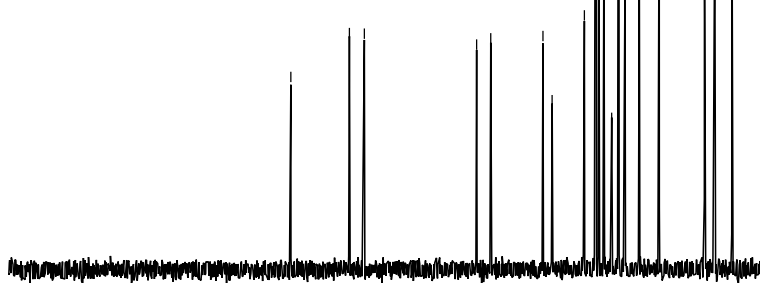

$00 \quad 190$

$180 \quad 170$

160

150

$40 \quad 130$

120

110

$100 \quad 90$

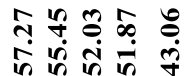

$\stackrel{\infty}{\sim}$

$1<1$ 


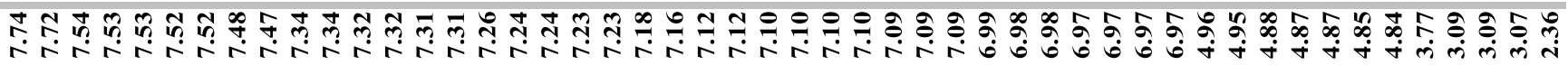

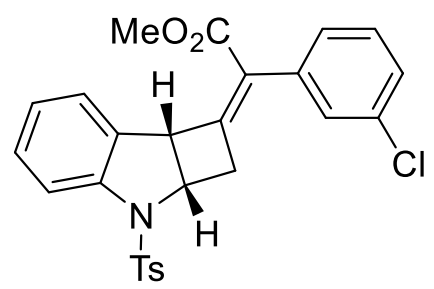

(Z)-3aq

$\left(500 \mathrm{MHz}\right.$; in $\left.\mathrm{CDCl}_{3}\right)$

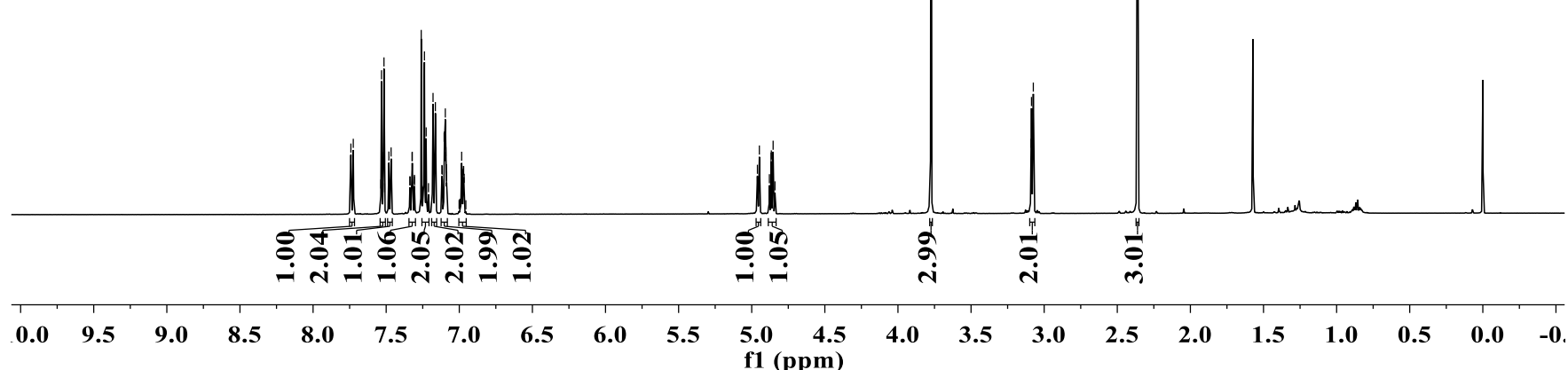

ڤ.

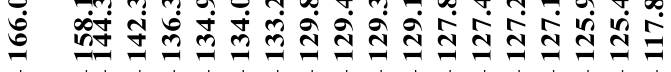

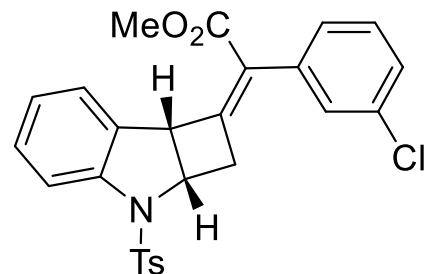

(Z)-3aq

$\left(125 \mathrm{MHz}\right.$; in $\mathrm{CDCl}_{3}$ )

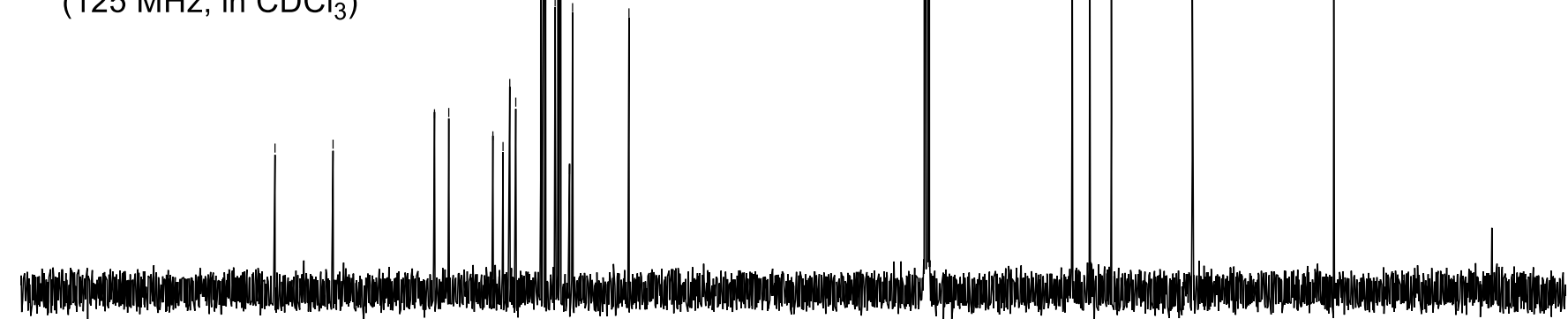

$\begin{array}{lllllllllll}190 & 180 & 170 & 160 & 150 & 140 & 130 & 120 & 110 & 100 & 90\end{array}$

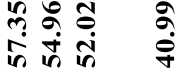

ำ 
<smiles>[3H]N1c2ccccc2[C@@H]2/C(=C(/C(=O)OC)c3cccc(Cl)c3)C[C@H]21</smiles>

(E)-3aq

$\left(500 \mathrm{MHz}\right.$; in $\left.\mathrm{CDCl}_{3}\right)$

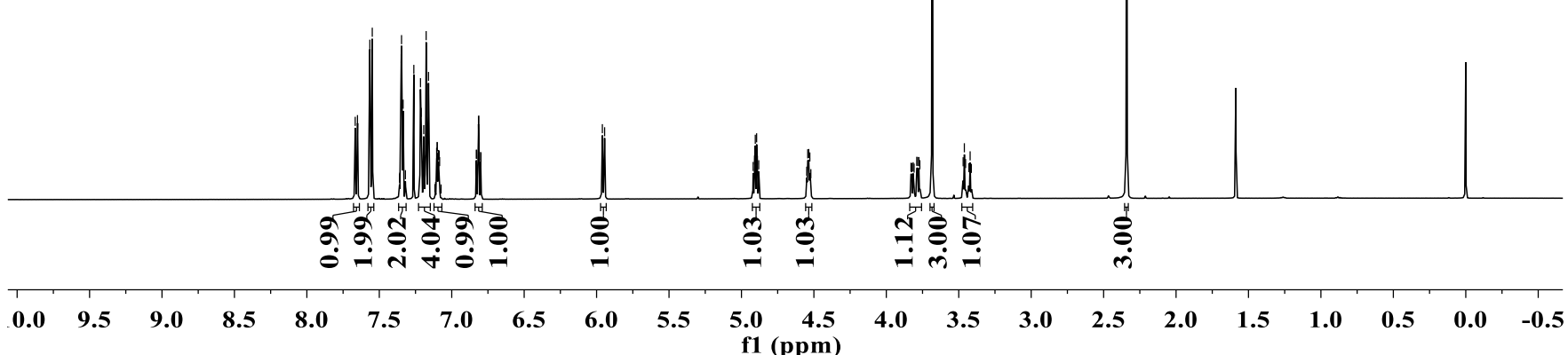
กั

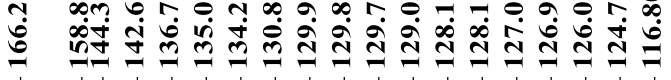<smiles>COC(=O)/C(=C1\C[C@@H]2[C@@H]1c1ccccc1N2[As])c1cccc(Cl)c1</smiles>

(E)-3aq

$\left(125 \mathrm{MHz}\right.$; in $\mathrm{CDCl}_{3}$ )

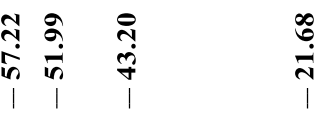




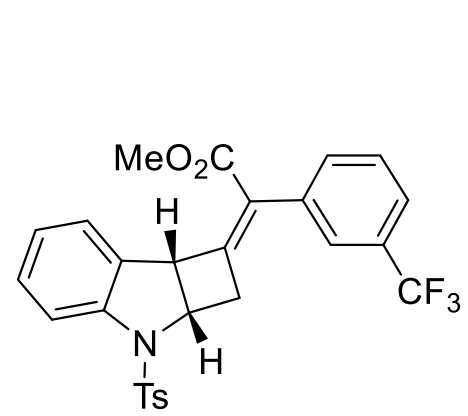

(Z)-3ar

$\left(500 \mathrm{MHz}\right.$; in $\left.\mathrm{CDCl}_{3}\right)$

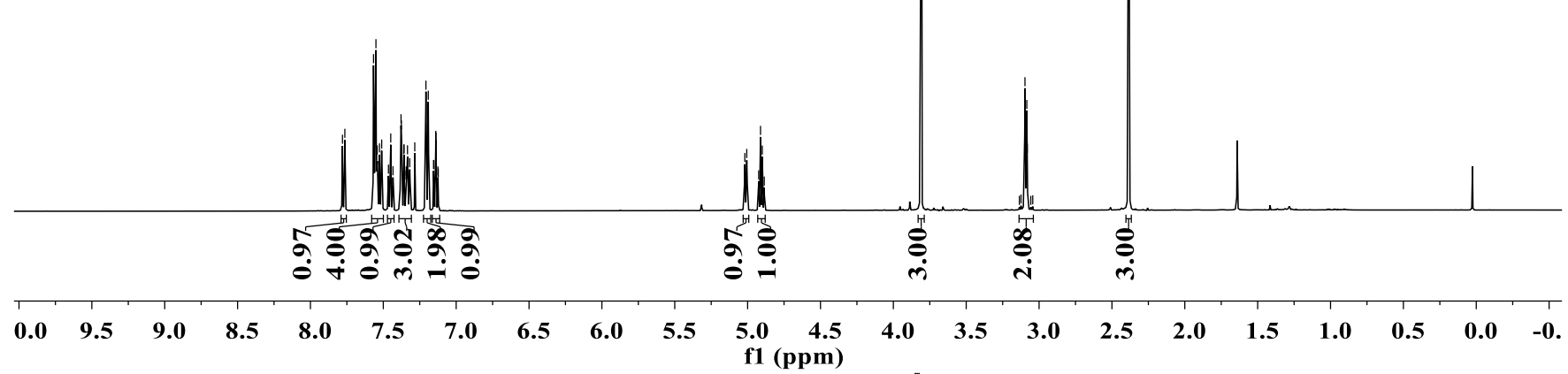

бำ

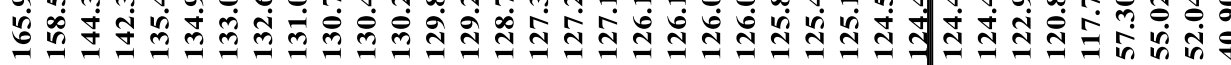

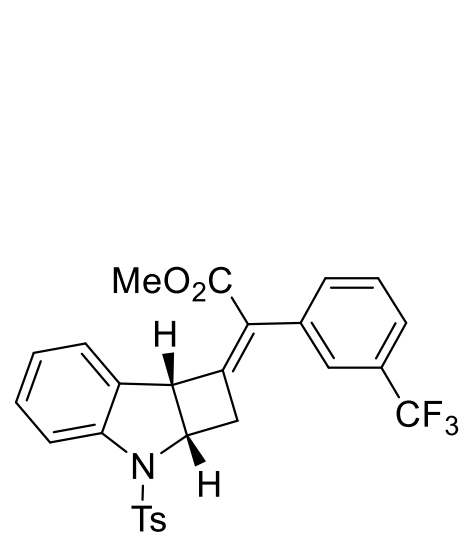

(Z)-3ar

$\left(125 \mathrm{MHz}\right.$; in $\mathrm{CDCl}_{3}$ )

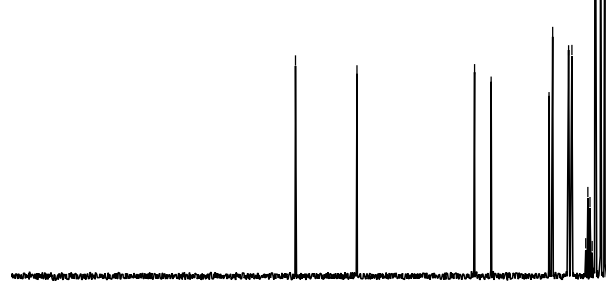


<smiles>CC(=O)/C(=C1/C[C@H]2[C@H]1c1ccccc1N2[13CH3])c1cccc(C(F)(F)F)c1</smiles>

(E)-3ar

$\left(500 \mathrm{MHz}\right.$; in $\left.\mathrm{CDCl}_{3}\right)$

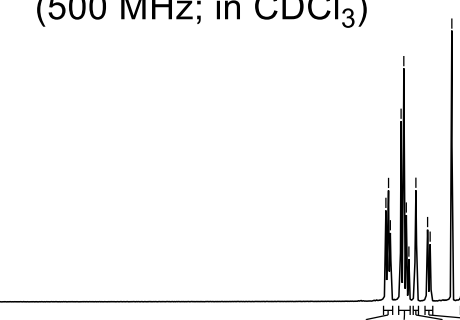
î.

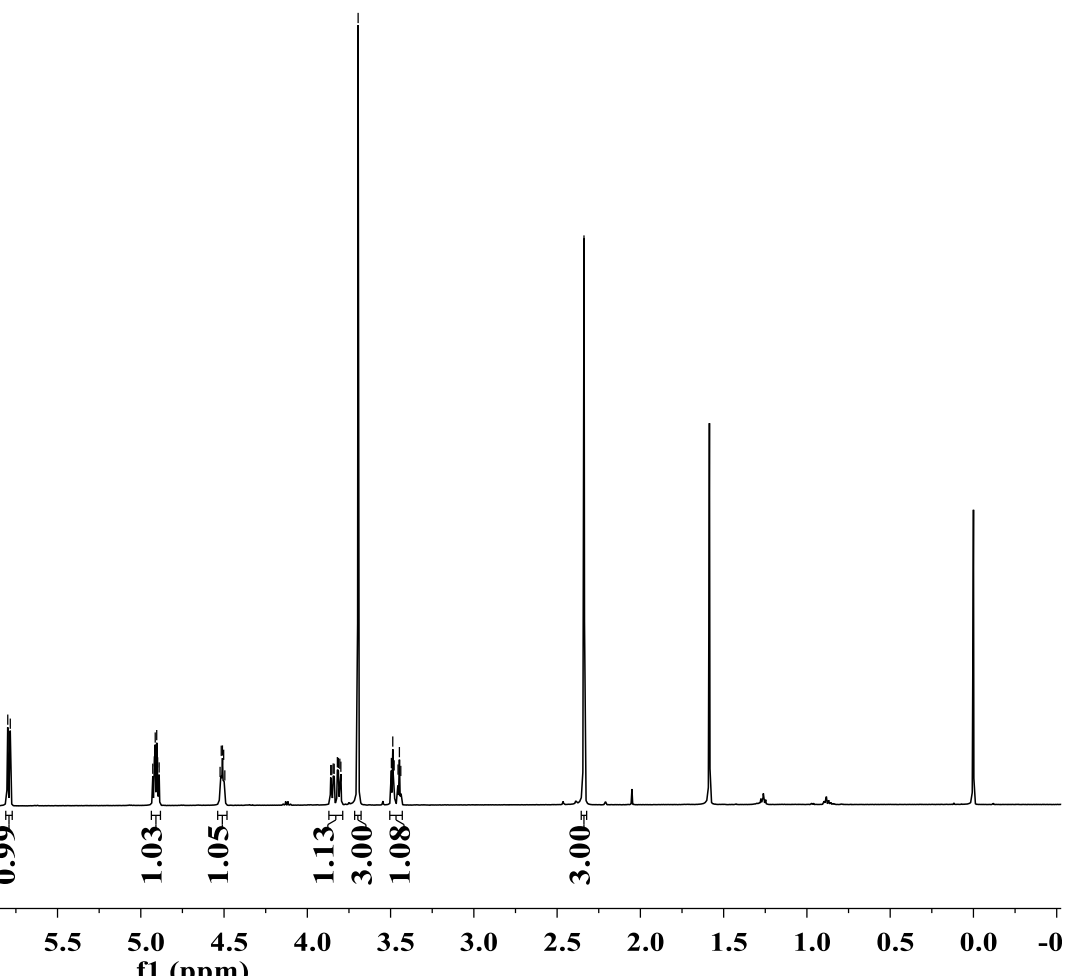

กิ

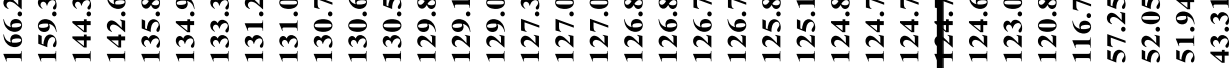

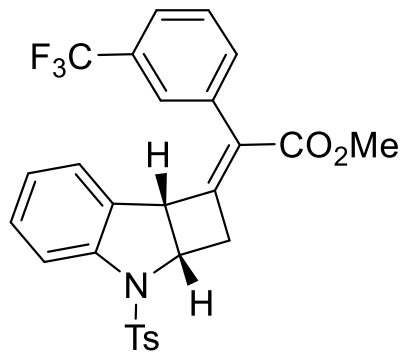

(E)-3ar

$\left(125 \mathrm{MHz}\right.$; in $\mathrm{CDCl}_{3}$ ) 
<smiles>COC(=C1C[C@@H]2[C@@H]1[C@@H]2c1ccccc1N(F)S)c1ccccc1C</smiles>

(Z)-3as

$\left(500 \mathrm{MHz}\right.$; in $\left.\mathrm{CDCl}_{3}\right)$
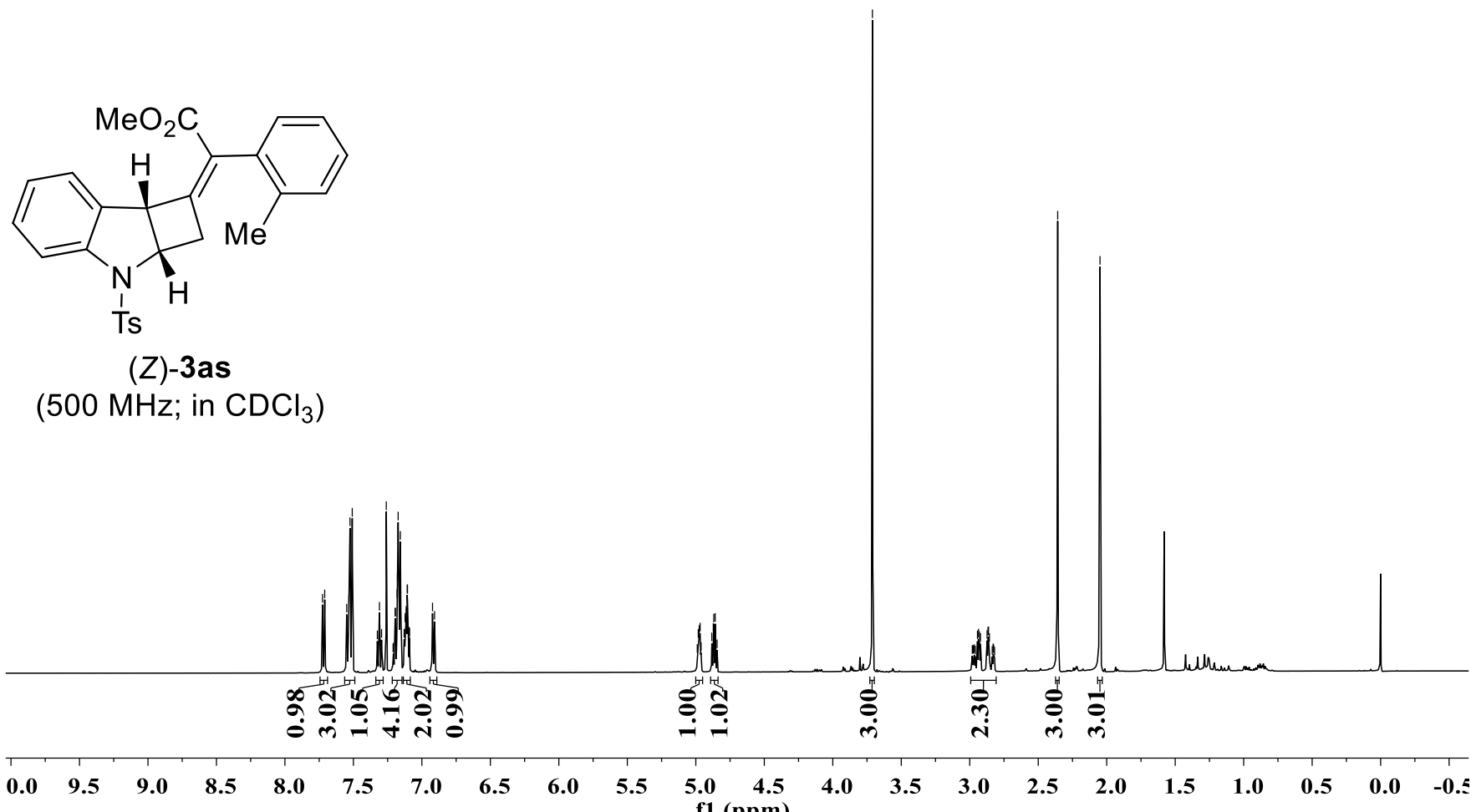

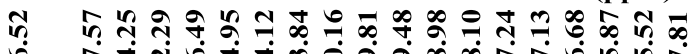

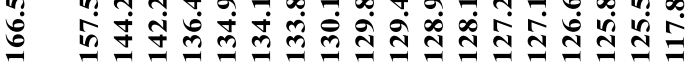

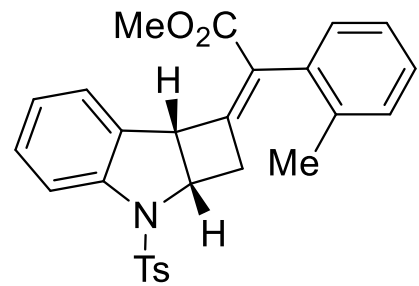

(Z)-3as

(125 $\mathrm{MHz}$; in $\mathrm{CDCl}_{3}$ )

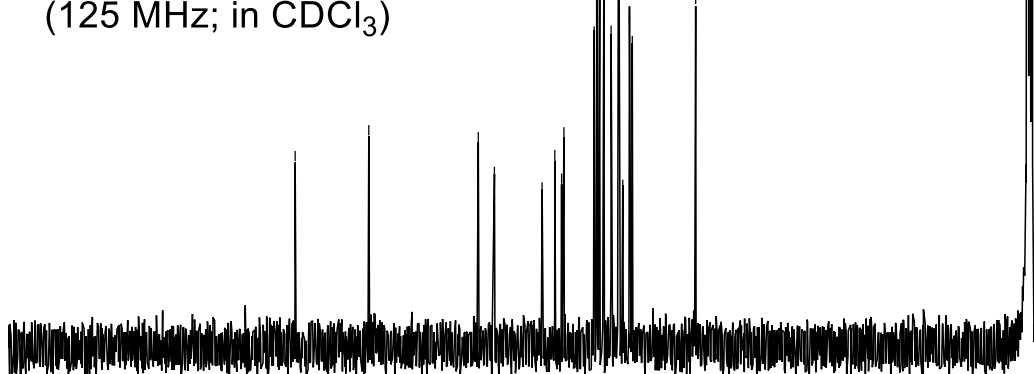

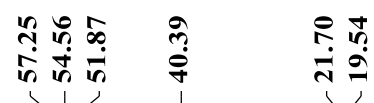


<smiles>CC(=O)/C(=C1/C[C@H]2[C@H]1c1ccccc1N2[As])c1ccccc1C</smiles>

(E)-3as

$\left(500 \mathrm{MHz}\right.$; in $\left.\mathrm{CDCl}_{3}\right)$

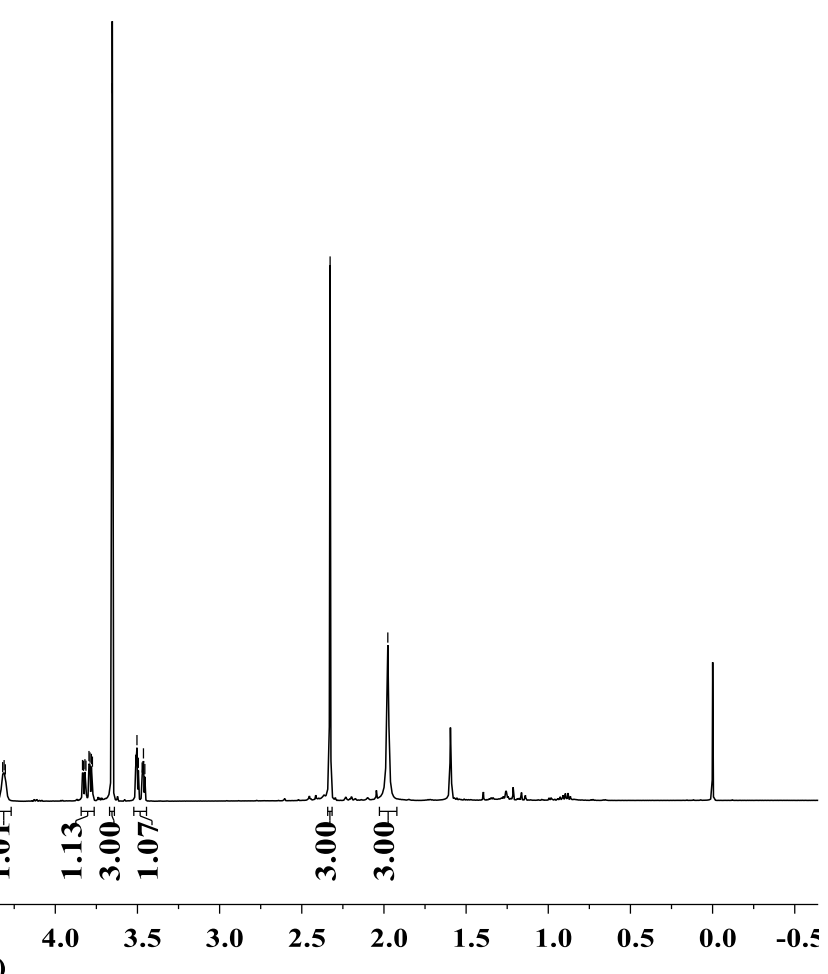<smiles>CC(=O)/C(=C1/C[C@H]2[C@H]1c1ccccc1N2[As])c1ccccc1C</smiles>

(E)-3as

$\left(125 \mathrm{MHz}\right.$; in $\left.\mathrm{CDCl}_{3}\right)$

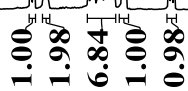

芒

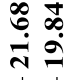


<smiles>[3H]N1c2ccccc2[C@H]2C(=C(OC)c3ccc(Cl)c(Cl)c3)C[C@H]21</smiles>

(Z)-3at

(500 MHz; in $\mathrm{CDCl}_{3}$ )

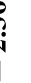

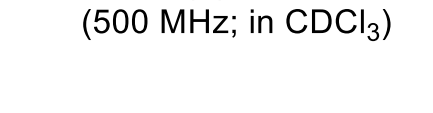

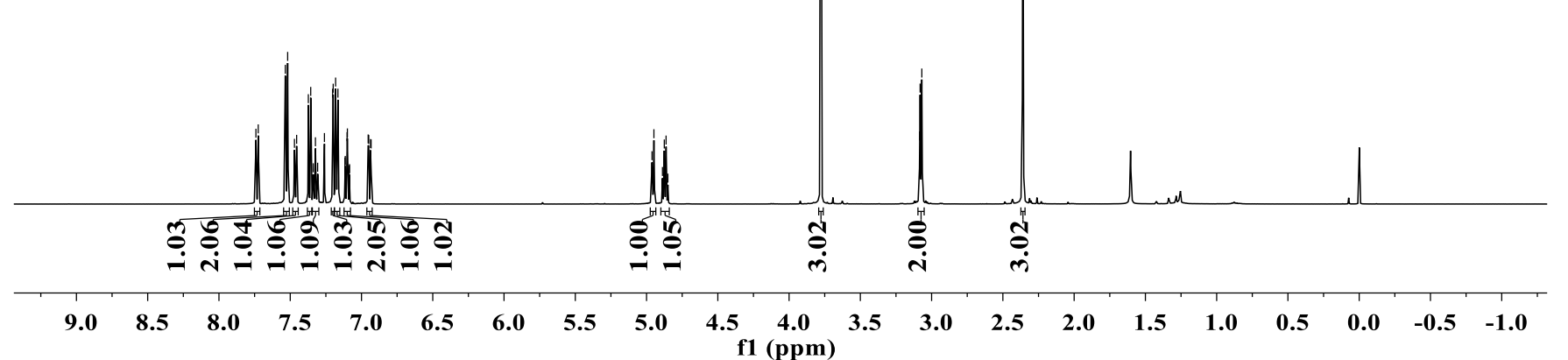

궁

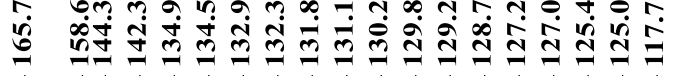

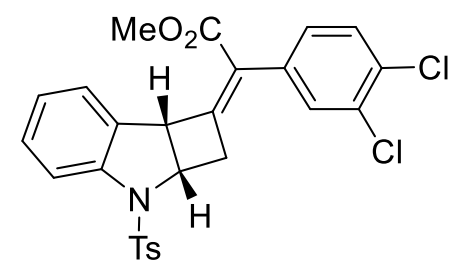

(Z)-3at

$\left(125 \mathrm{MHz}\right.$; in $\mathrm{CDCl}_{3}$ )

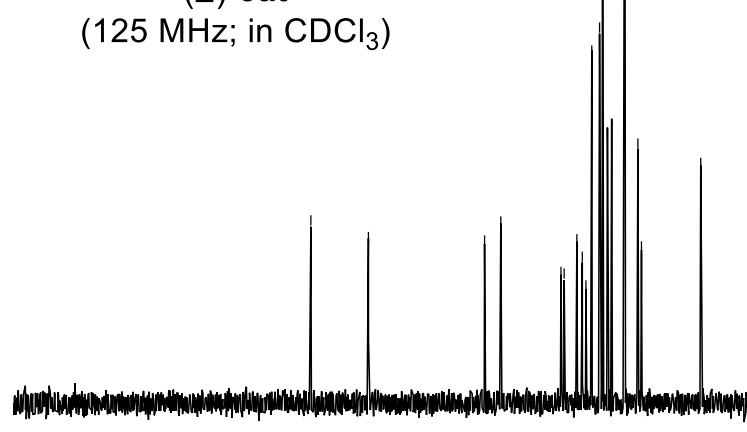


<smiles>[3H]N1c2ccccc2[C@H]2/C(=C(\C(=O)OC)c3ccc(Cl)c(Cl)c3)C[C@@H]21</smiles>

(E)-3at

$\left(500 \mathrm{MHz}\right.$; in $\left.\mathrm{CDCl}_{3}\right)$

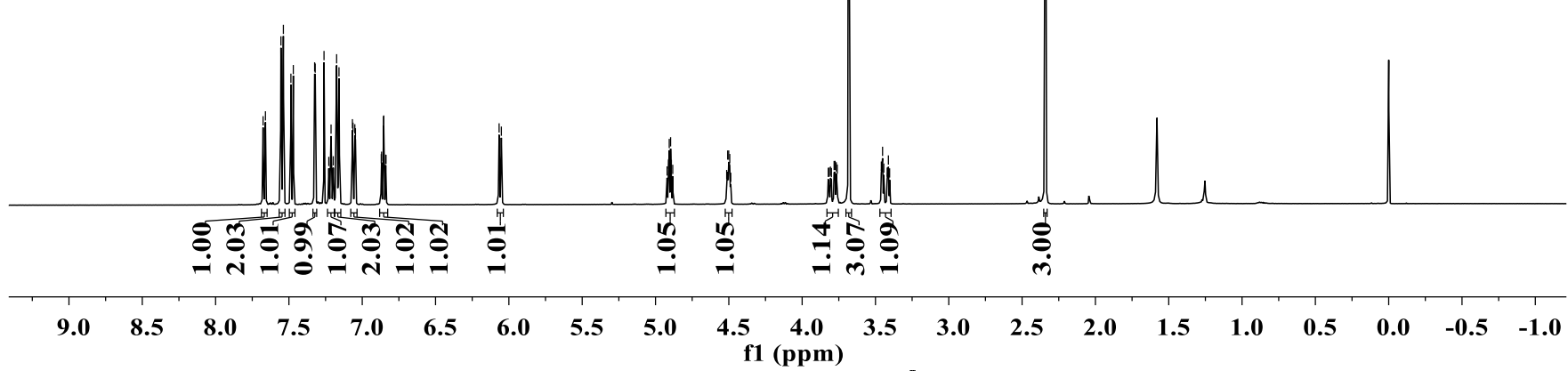
ใิ

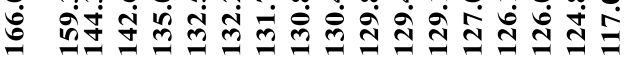<smiles>[3H]N1c2ccccc2[C@H]2/C(=C(\C(=O)OC)c3ccc(Cl)c(Cl)c3)C[C@@H]21</smiles>

(E)-3at

(125 MHz; in $\mathrm{CDCl}_{3}$ ) 
<smiles>COC(C)=C1C[C@H]2[C@H]1c1ccccc1N2[13S]</smiles>

(Z)-3au

$\left(500 \mathrm{MHz}\right.$; in $\left.\mathrm{CDCl}_{3}\right)$

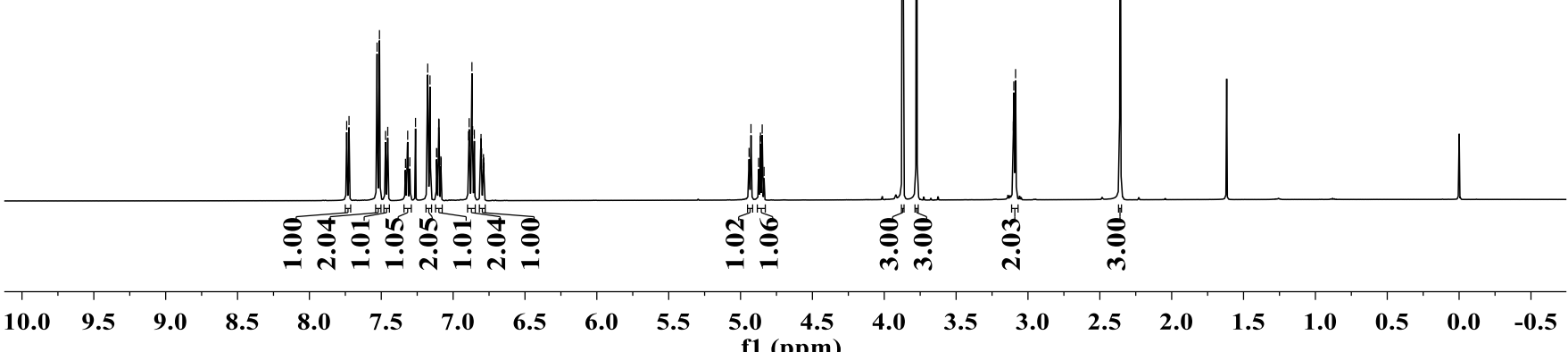

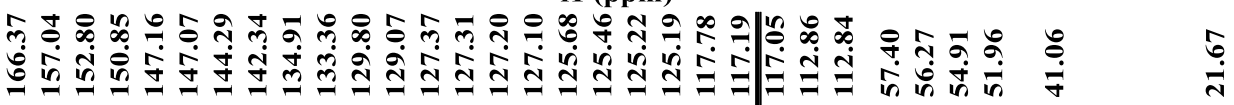

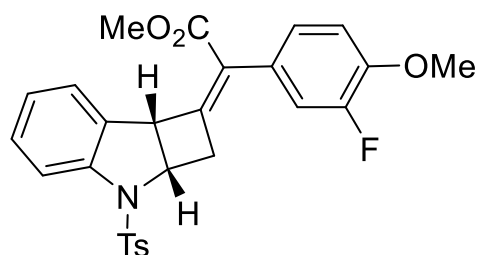

(Z)-3au

$\left(125 \mathrm{MHz}\right.$; in $\left.\mathrm{CDCl}_{3}\right)$ 


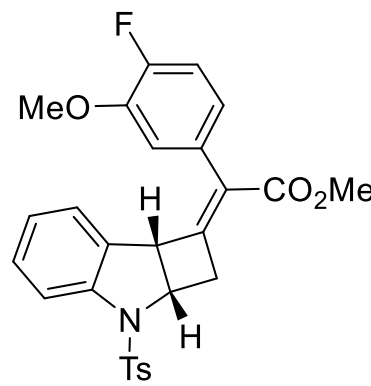

(E)-3au

$\left(500 \mathrm{MHz}\right.$; in $\mathrm{CDCl}_{3}$ )
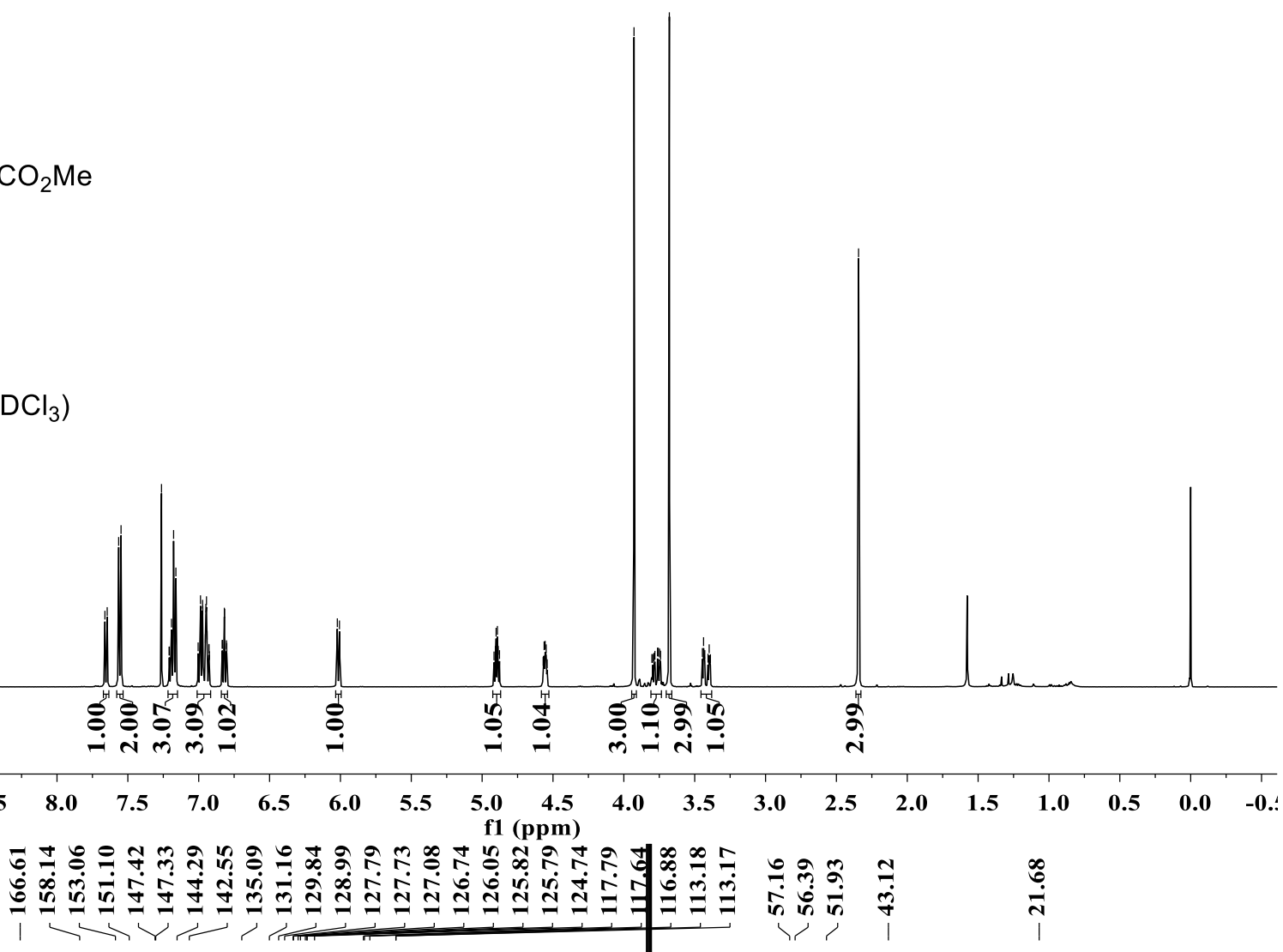<smiles>COc1cc(/C(C(C)=O)=C2/C[C@H]3[C@H]2c2ccccc2N3C)ccc1F</smiles>

(E)-3au

(125 MHz; in $\mathrm{CDCl}_{3}$ ) 


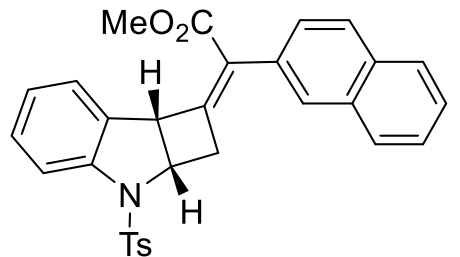

(Z)-3av

$\left(500 \mathrm{MHz} ;\right.$ in $\left.\mathrm{CDCl}_{3}\right)$

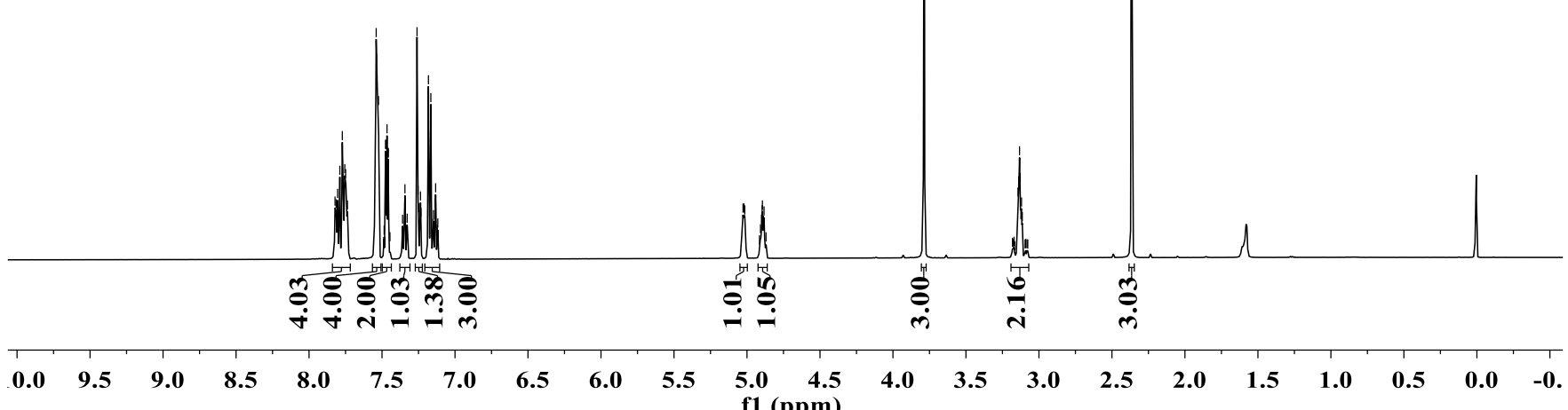

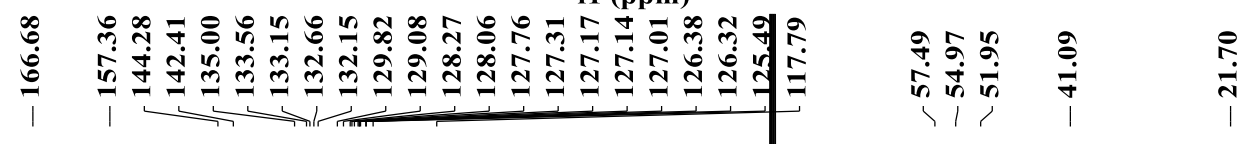

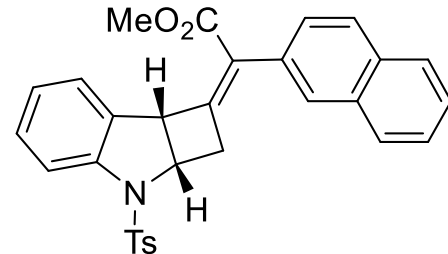

(Z)-3av

$\left(125 \mathrm{MHz}\right.$; in $\left.\mathrm{CDCl}_{3}\right)$ 


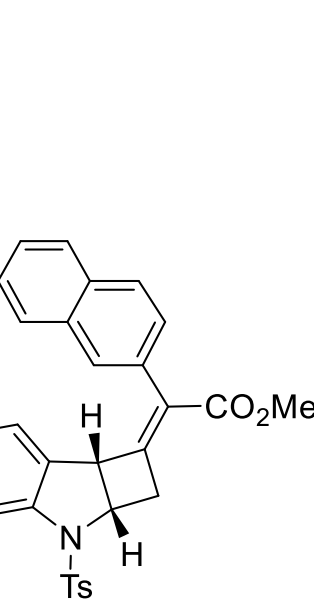

(E)-3av

$\left(500 \mathrm{MHz}\right.$; in $\left.\mathrm{CDCl}_{3}\right)$

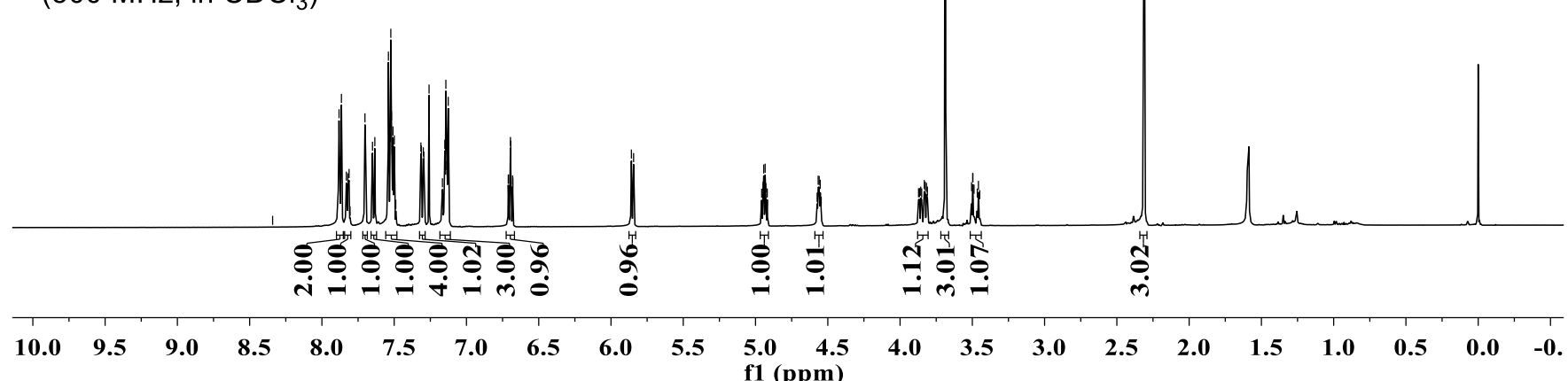

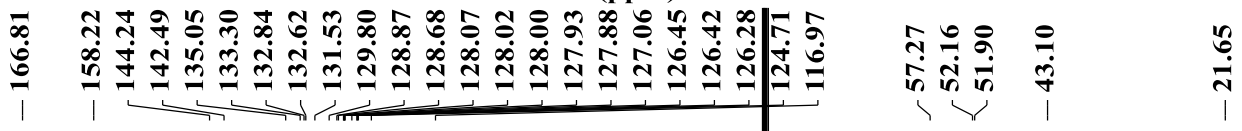

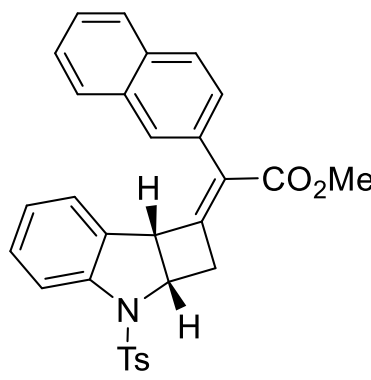

$(E)-3 a v$

(125 MHz; in $\mathrm{CDCl}_{3}$ )

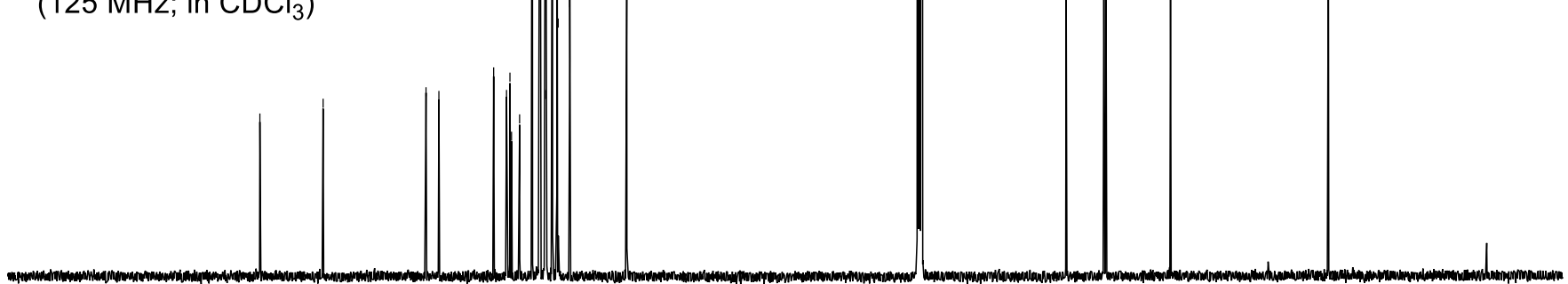




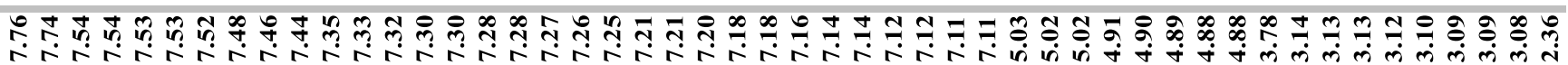

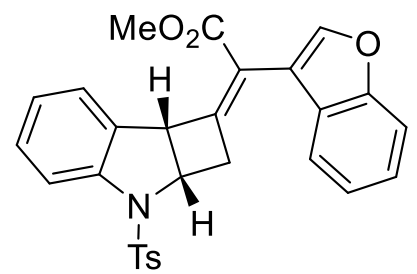

(Z)-3aw

$\left(500 \mathrm{MHz}\right.$; in $\left.\mathrm{CDCl}_{3}\right)$

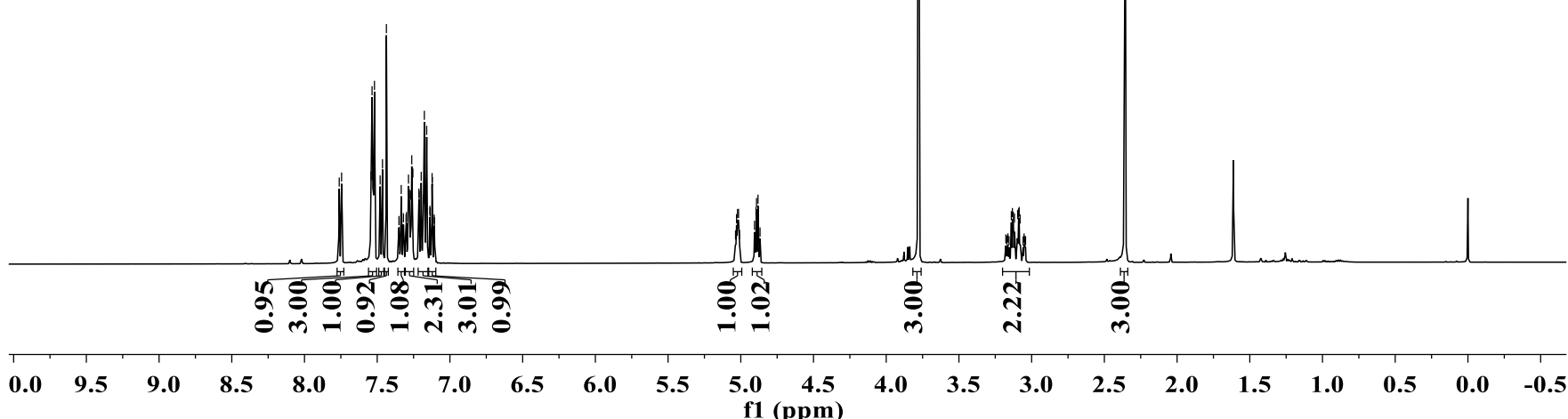

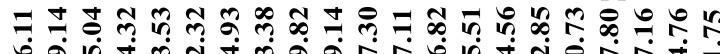

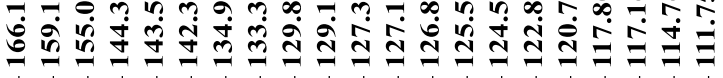

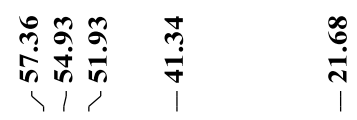

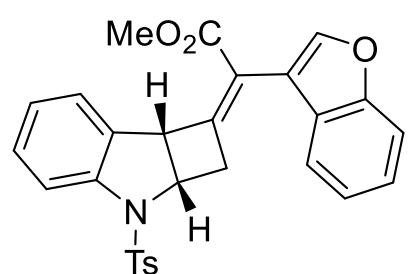

(Z)-3aw

$\left(125 \mathrm{MHz}\right.$; in $\left.\mathrm{CDCl}_{3}\right)$

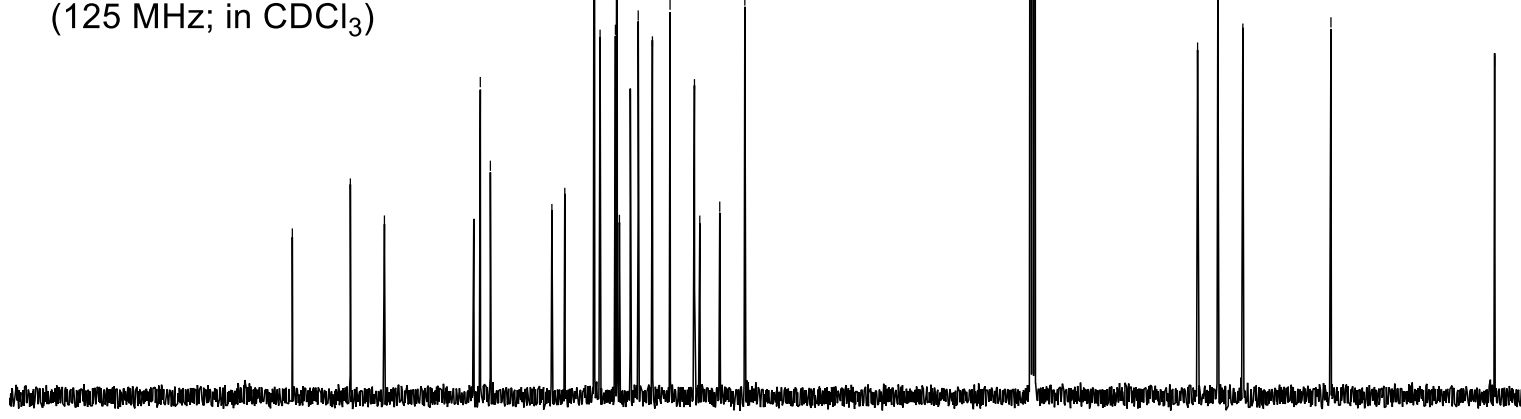


<smiles>CC(=O)/C(=C1/C[C@H]2[C@H]1c1ccccc1N2[13CH3])c1coc2ccccc12</smiles>

(E)-3aw

$\left(500 \mathrm{MHz}\right.$; in $\mathrm{CDCl}_{3}$ )<smiles>CC(=O)/C(=C1/C[C@H]2[C@H]1c1ccccc1N2[13CH3])c1coc2ccccc12</smiles>

(E)-3aw

$\left(125 \mathrm{MHz}\right.$; in $\mathrm{CDCl}_{3}$ )

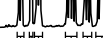

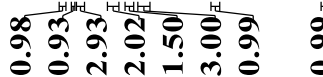

0.0

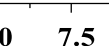

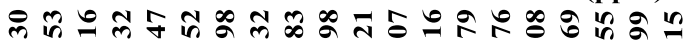

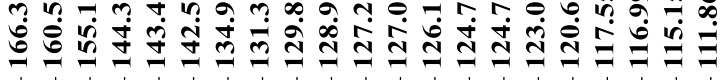

乌ั

4.0

$3.5 \quad 3.0$

1.

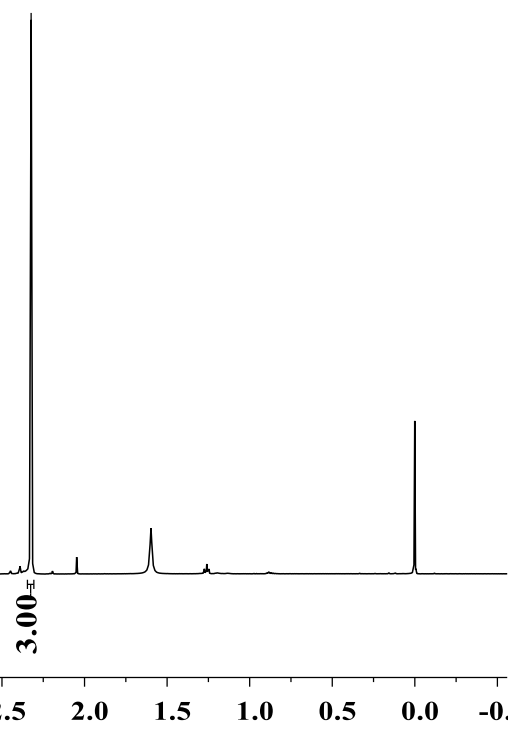

กิษ

in

$\stackrel{\leftrightarrow}{\stackrel{4}{*}}$ 


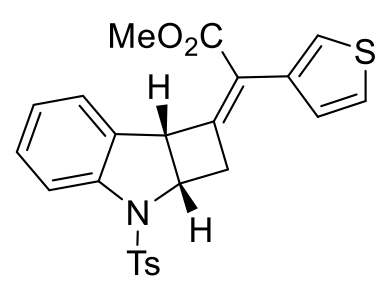

(Z)-3ax

$\left(500 \mathrm{MHz}\right.$; in $\mathrm{CDCl}_{3}$ )

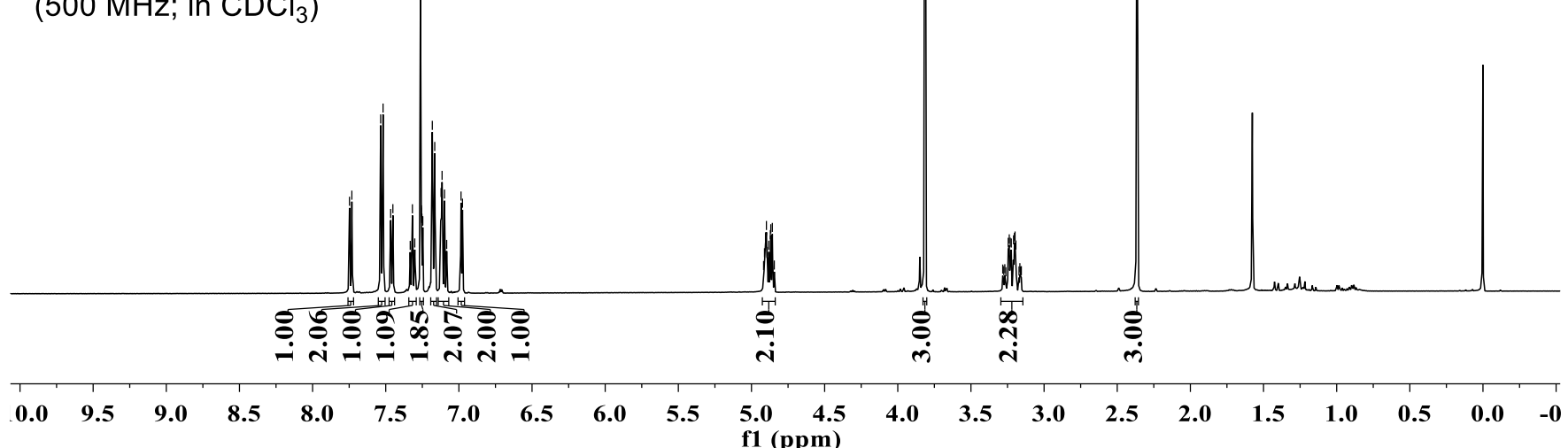

ๆ

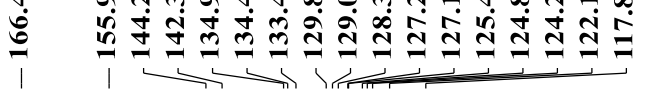

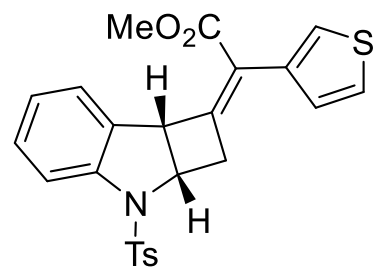

(Z)-3ax

$\left(125 \mathrm{MHz}\right.$; in $\mathrm{CDCl}_{3}$ )

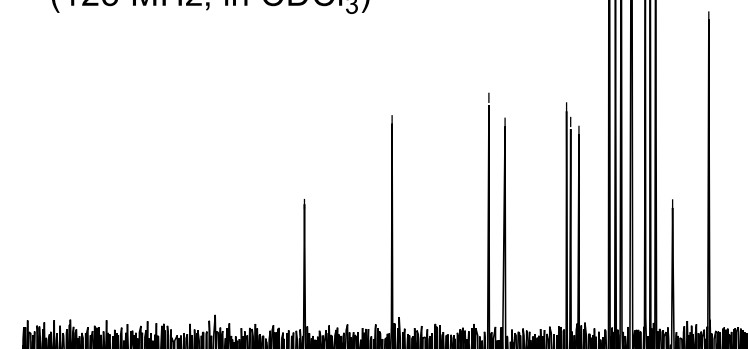




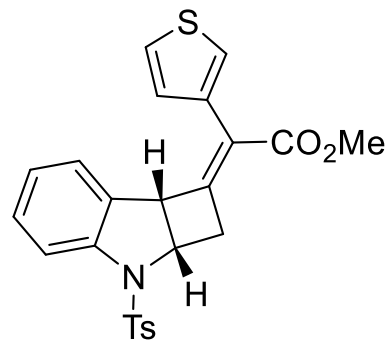

(E)-3ax

(500 MHz; in $\mathrm{CDCl}_{3}$ )

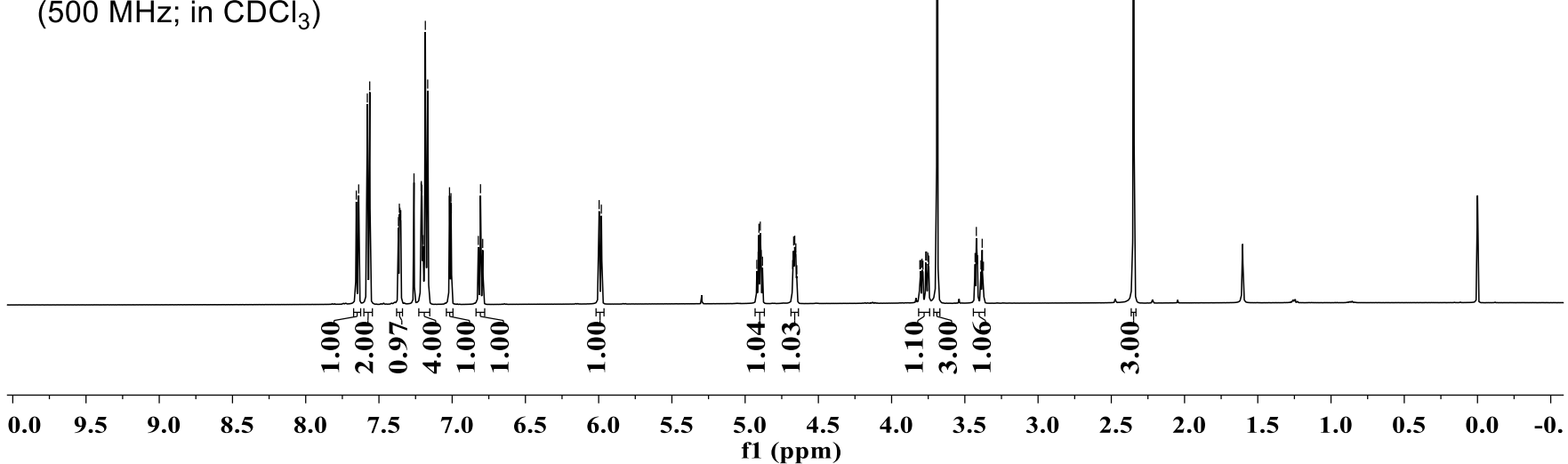
Бี 至

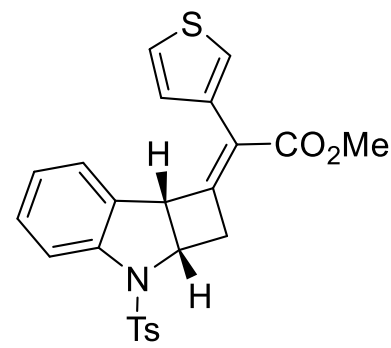

(E)-3ax

(125 MHz; in $\mathrm{CDCl}_{3}$ )

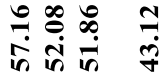

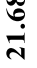




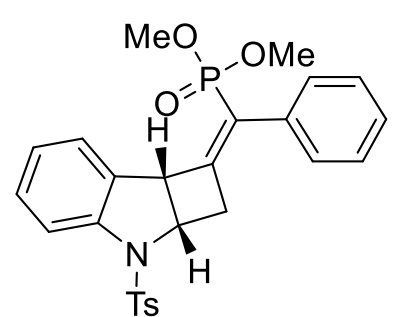

(Z)-3ay
(500 MHz; in $\mathrm{CDCl}_{3}$ )

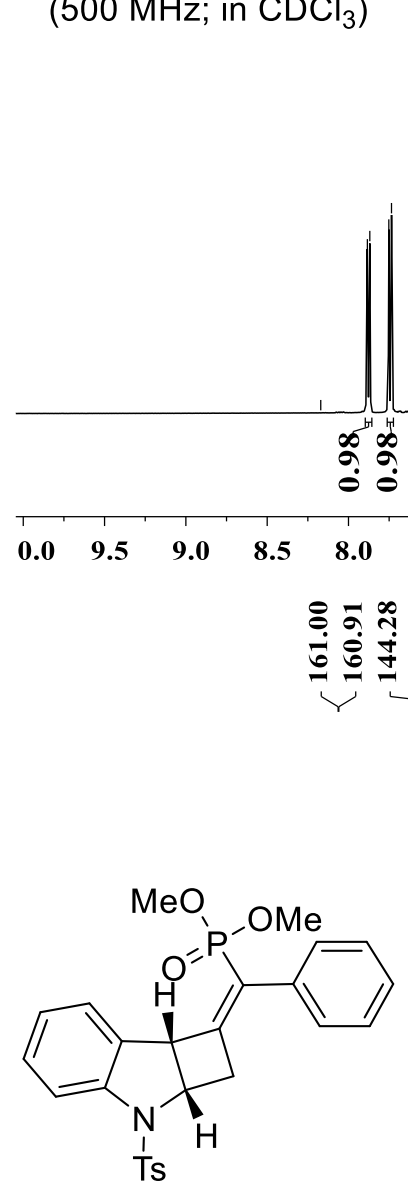

(Z)-3ay

(125 MHz; in $\mathrm{CDCl}_{3}$ )

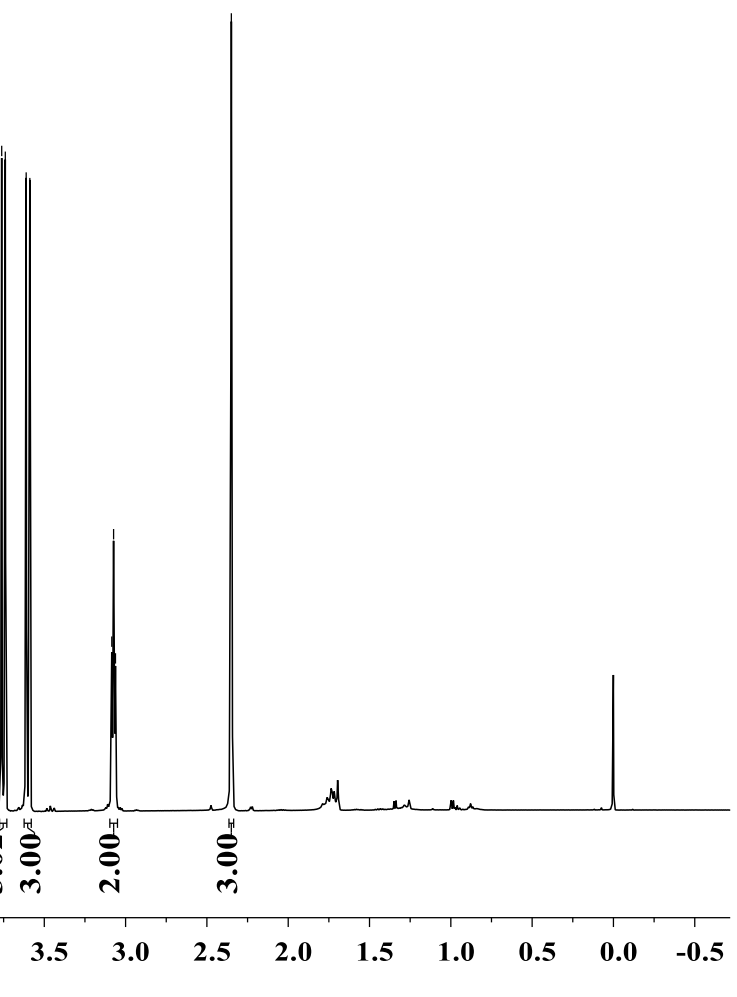

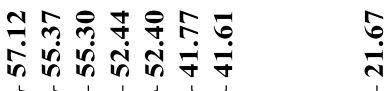

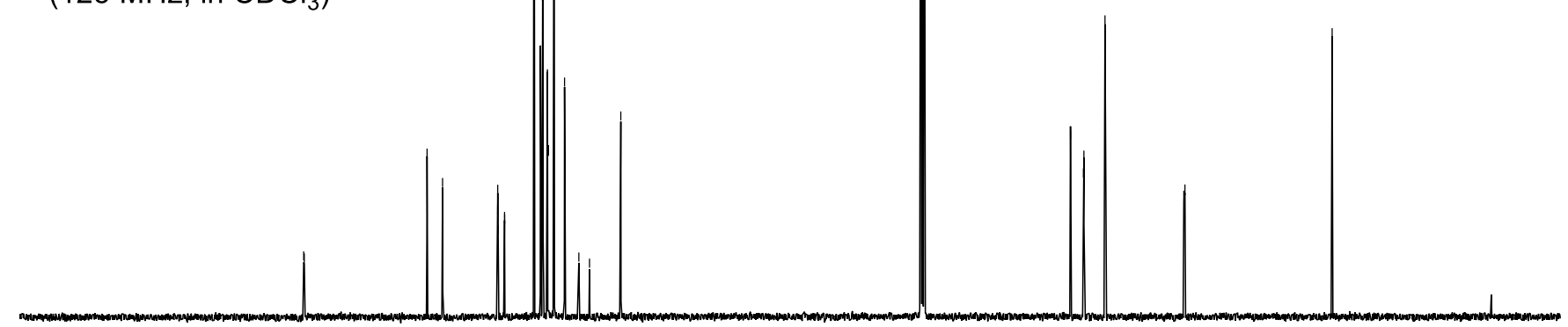




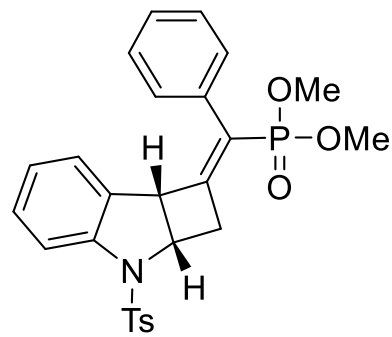

$(E)$-3ay

$\left(500 \mathrm{MHz}\right.$; in $\mathrm{CDCl}_{3}$ )

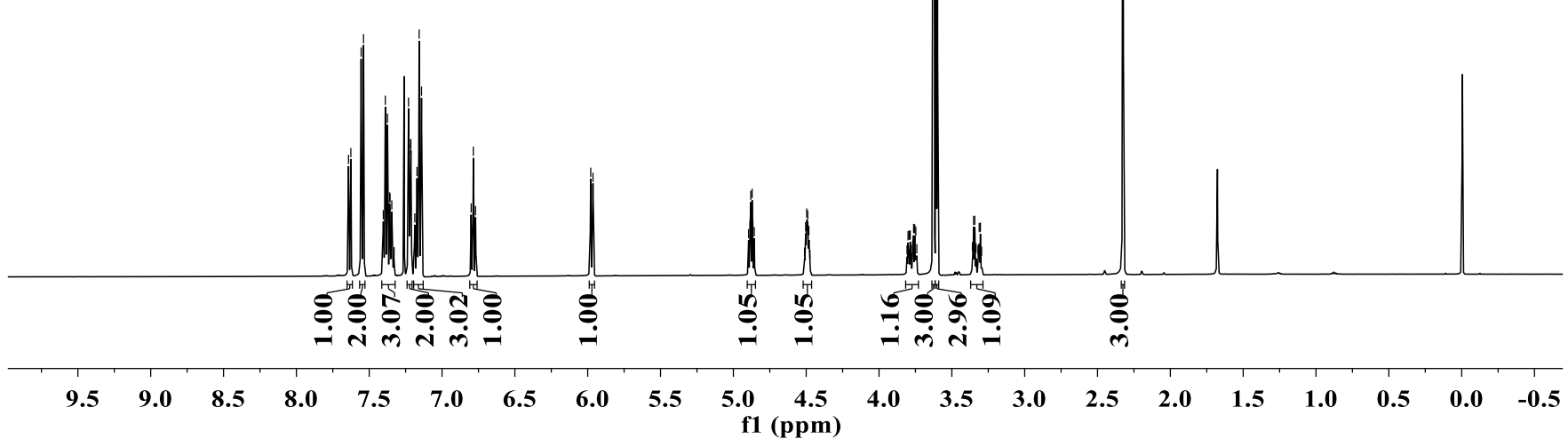

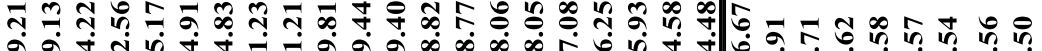

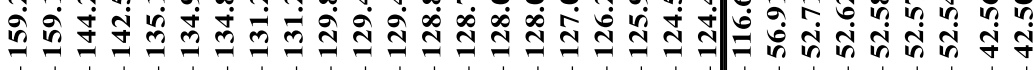

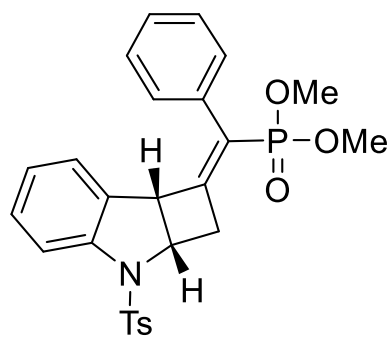

(E)-3ay

(125 MHz; in $\mathrm{CDCl}_{3}$ )

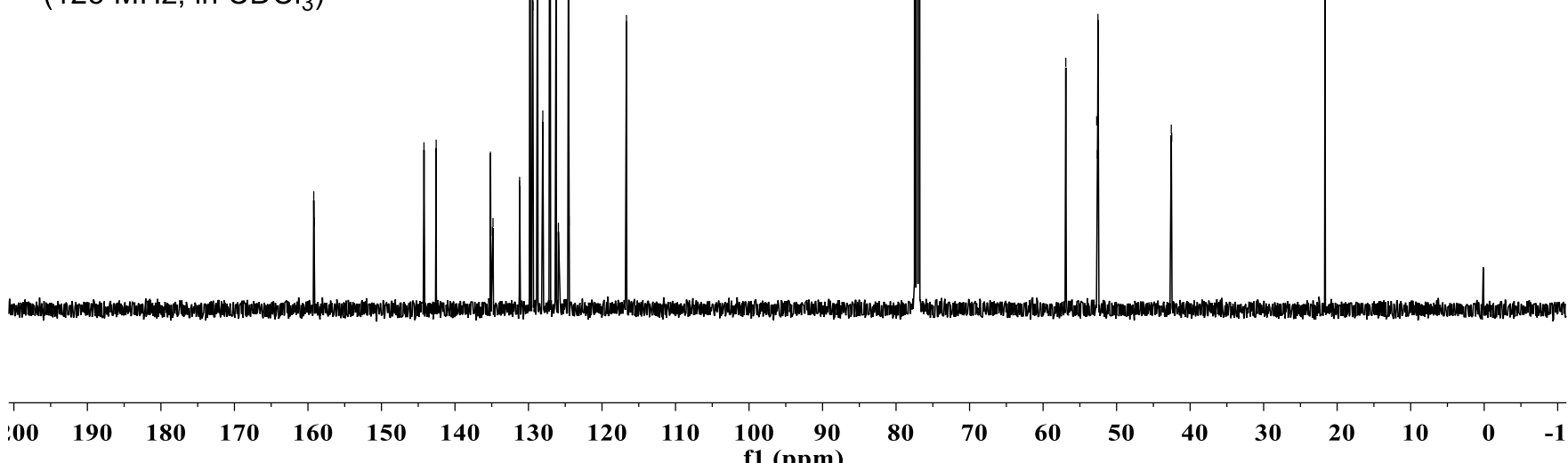


<smiles>O=C1Nc2ccccc2/C1=C1\C[C@H]2[C@H]1c1ccccc1N2Cc1ccccc1</smiles>

(Z)-3az

$\left(500 \mathrm{MHz}\right.$; in $\mathrm{CDCl}_{3}$ )

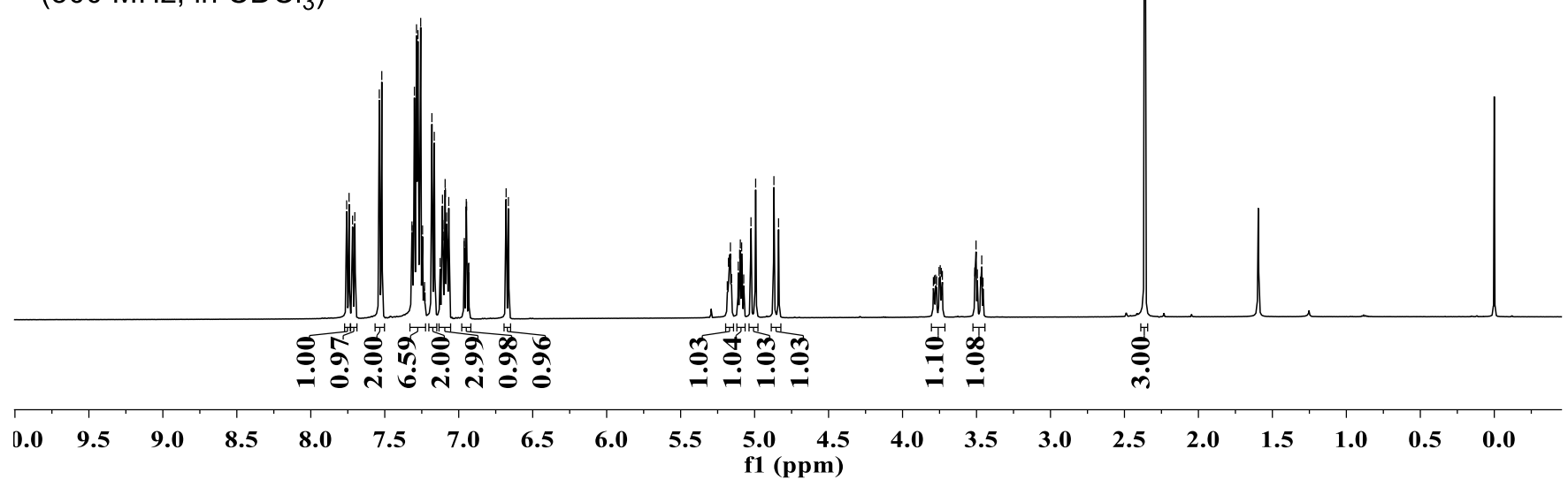

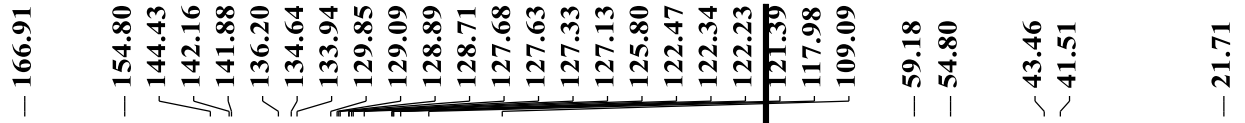<smiles>O=C1Nc2ccccc2/C1=C1\C[C@H]2[C@H]1c1ccccc1N2Cc1ccccc1</smiles>

(Z)-3az

$\left(125 \mathrm{MHz}\right.$; in $\mathrm{CDCl}_{3}$ )

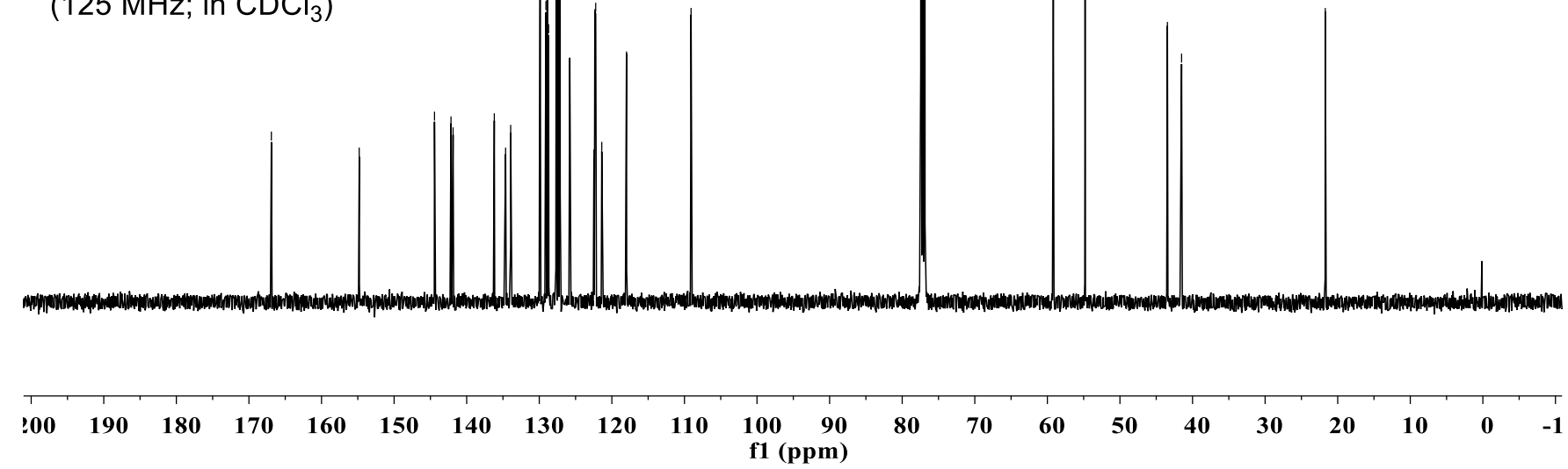




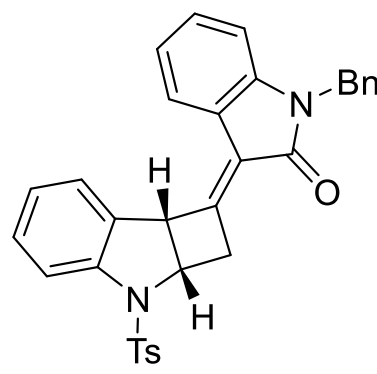

$(E)-3 a z$

$\left(500 \mathrm{MHz}\right.$; in $\left.\mathrm{CDCl}_{3}\right)$

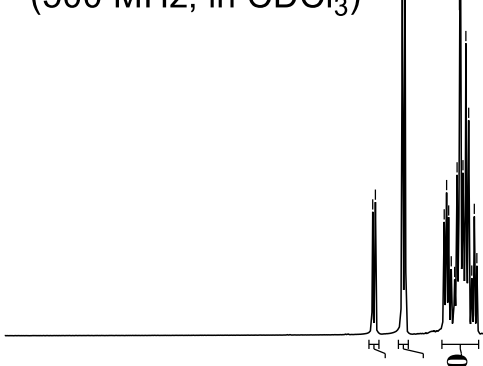

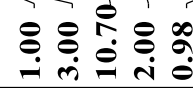

$\begin{array}{lllllllllllll}0.0 & 9.5 & 9.0 & 8.5 & 8.0 & 7.5 & 7.0 & 6.5 & 6.0 & 5.5 & 5.0 & 4.5\end{array}$

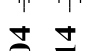

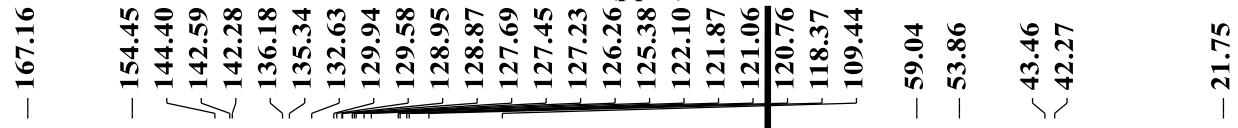

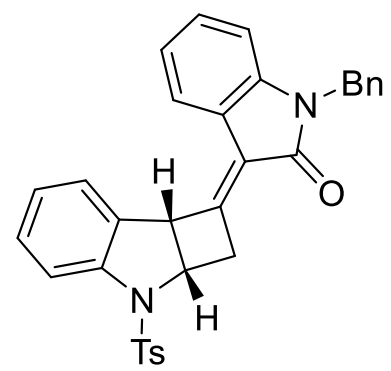

(E)-3az

(125 MHz; in $\mathrm{CDCl}_{3}$ )
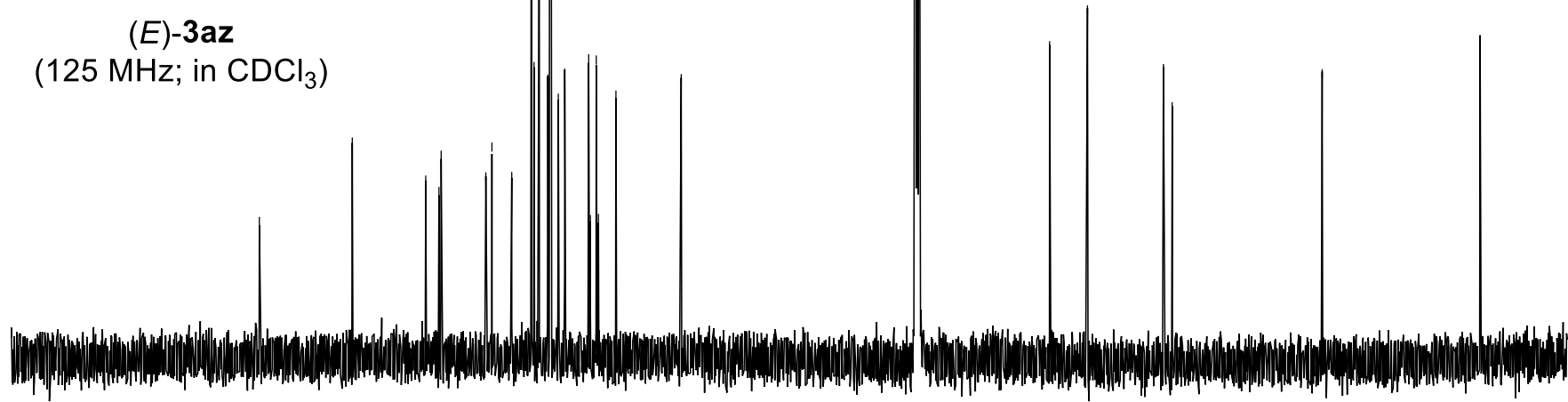


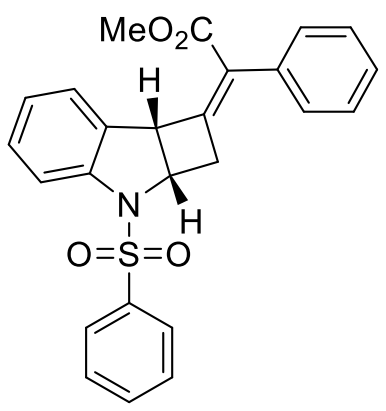

(Z)-3ba

$\left(500 \mathrm{MHz}\right.$; in $\left.\mathrm{CDCl}_{3}\right)$

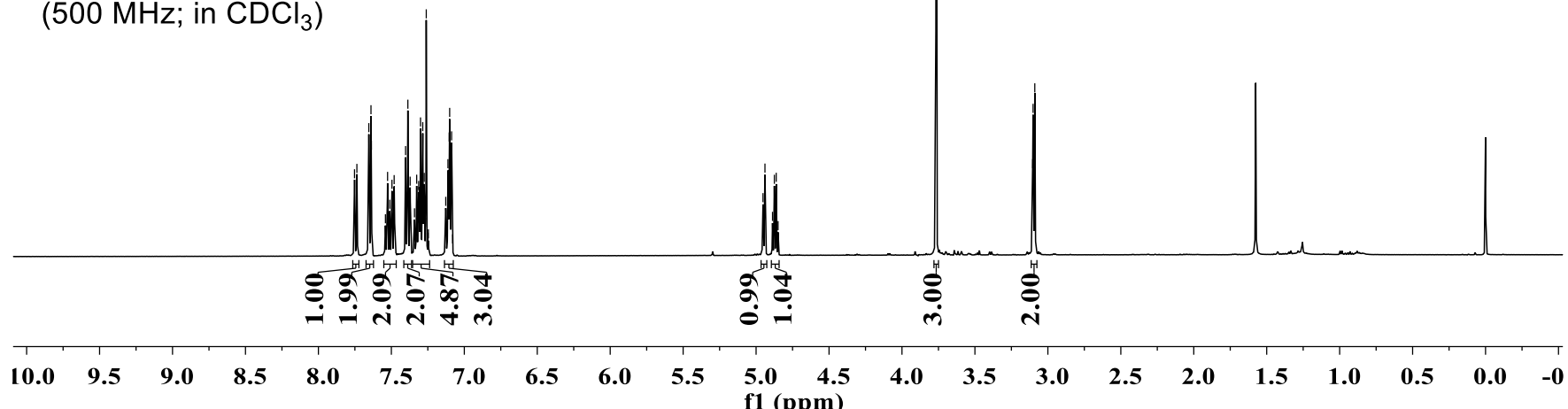

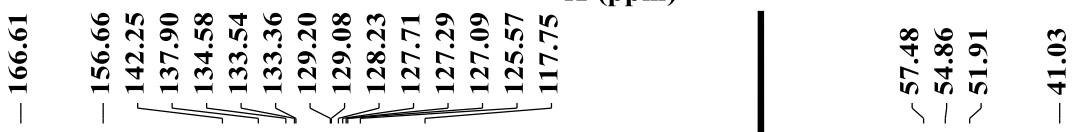

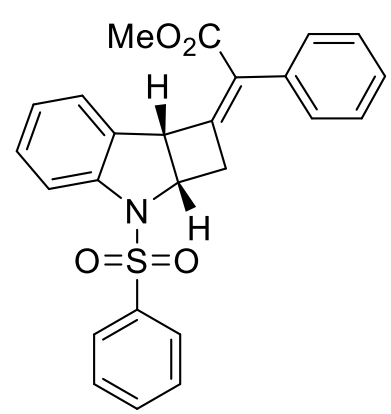

(Z)-3ba

$\left(125 \mathrm{MHz}\right.$; in $\mathrm{CDCl}_{3}$ )

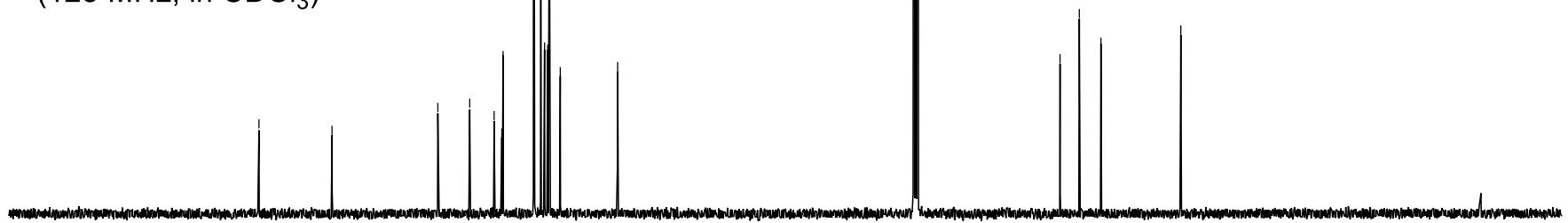


<smiles>CC(=O)C(=C1C[C@@H]2[C@@H]1[C@@H]1[C@@H]2c2ccccc2N1S(=O)(=O)c1ccccc1)c1ccccc1</smiles>

(E)-3ba

$\left(500 \mathrm{MHz}\right.$; in $\mathrm{CDCl}_{3}$ )<smiles>CC(=O)/C(=C1/C[C@@H]2[C@@H]1[C@@H]1[C@@H]2c2ccccc2N1S(=O)(=O)c1ccccc1)c1ccccc1</smiles>

(E)-3ba

$\left(125 \mathrm{MHz}\right.$; in $\mathrm{CDCl}_{3}$ )

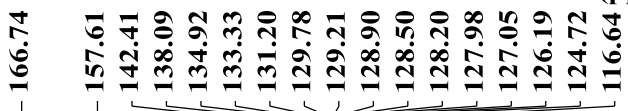

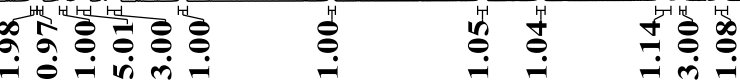

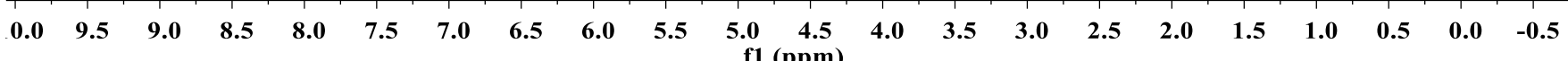

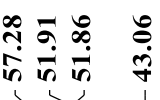

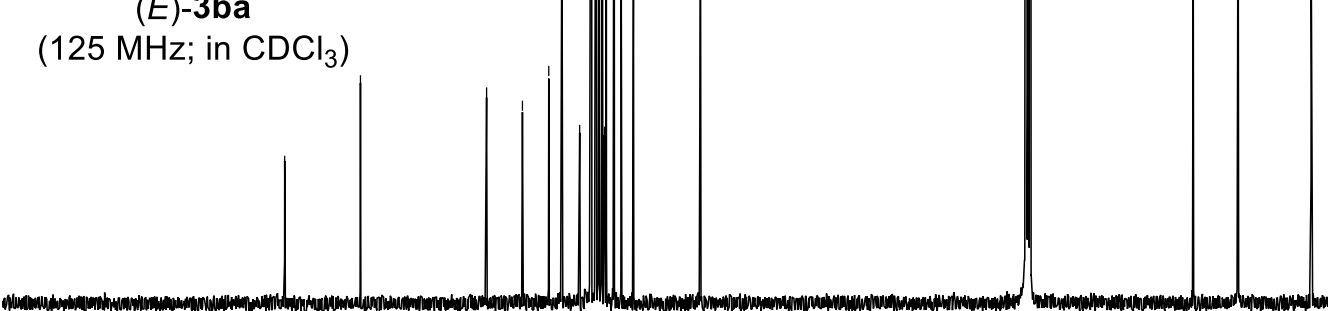




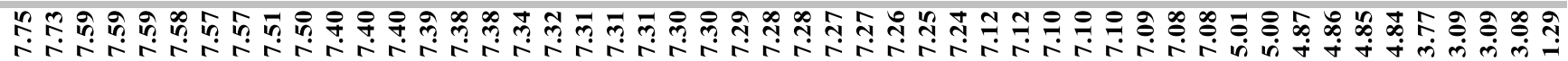<smiles>COC(=O)/C(=C1/C[C@H]2[C@H]1c1ccccc1N2S(=O)(=O)c1ccc(C(C)(C)C)cc1)c1ccccc1</smiles>

(Z)-3ca

$\left(500 \mathrm{MHz}\right.$; in $\left.\mathrm{CDCl}_{3}\right)$

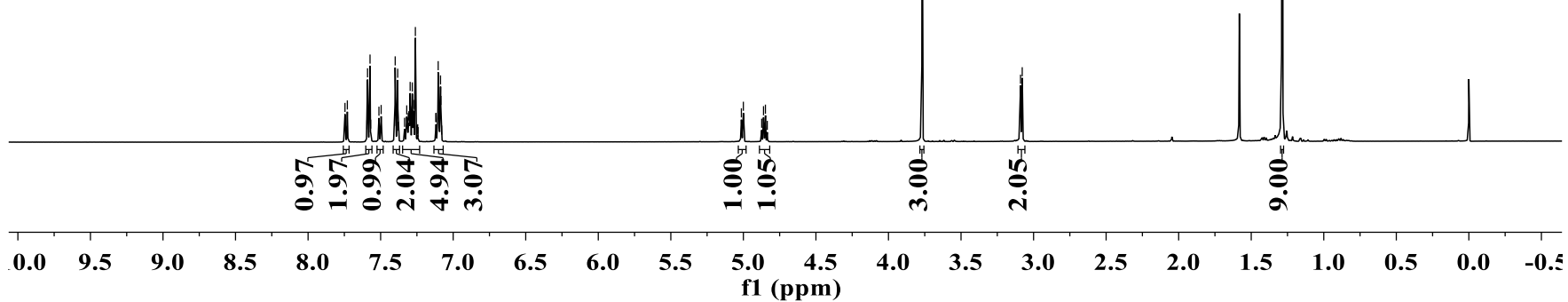

60.

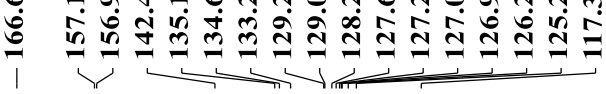<smiles>COC(=O)/C(=C1/C[C@@H]2[C@H]1c1ccccc1N2S(=O)(=O)c1ccc(C(C)(C)C)cc1)c1ccccc1</smiles>

(Z)-3ca

$\left(125 \mathrm{MHz}\right.$; in $\mathrm{CDCl}_{3}$ )

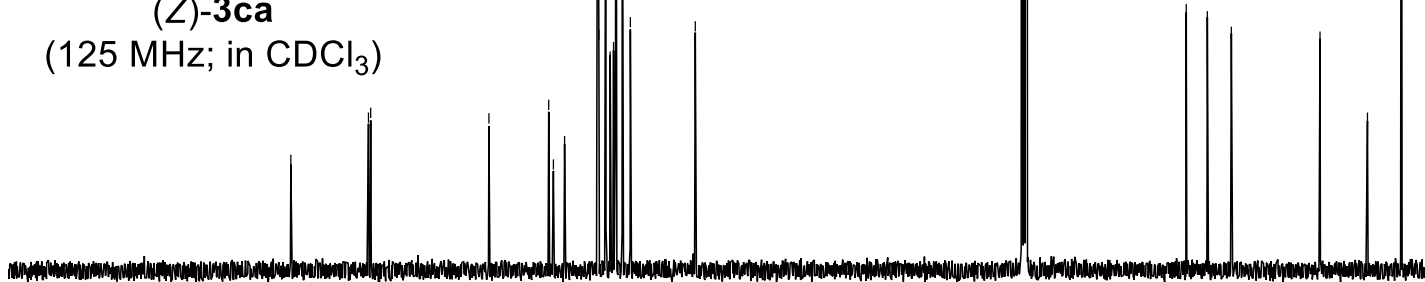


<smiles>COC(=O)/C(=C1/C[C@H]2[C@H]1c1ccccc1N2S(=O)(=O)c1ccc(C(C)(C)C)cc1)c1ccccc1</smiles>

$(E)-3 c a$

$\left(500 \mathrm{MHz} ;\right.$ in $\left.\mathrm{CDCl}_{3}\right)$

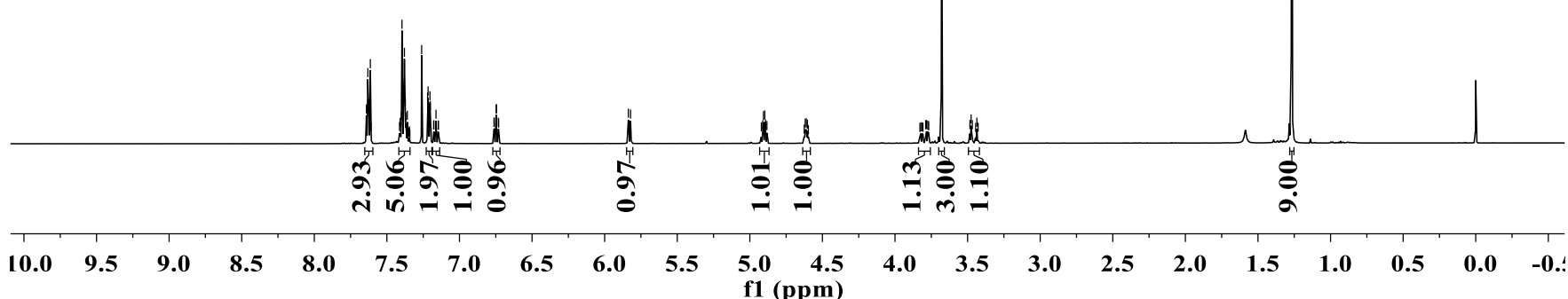<smiles>COC(=O)/C(=C1/C[C@H]2[C@H]1c1ccccc1N2S(=O)(=O)c1ccc(Br)cc1)c1ccccc1</smiles>

(E)-3ca

$\left(125 \mathrm{MHz}\right.$; in $\left.\mathrm{CDCl}_{3}\right)$

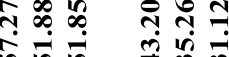

ने के 


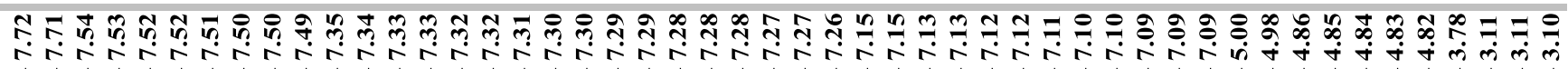<smiles>COC(=O)/C(=C1/C[C@H]2[C@H]1c1ccccc1N2S(=O)(=O)c1ccc(Br)cc1)c1ccccc1</smiles>

(Z)-3da

$\left(500 \mathrm{MHz}\right.$; in $\left.\mathrm{CDCl}_{3}\right)$

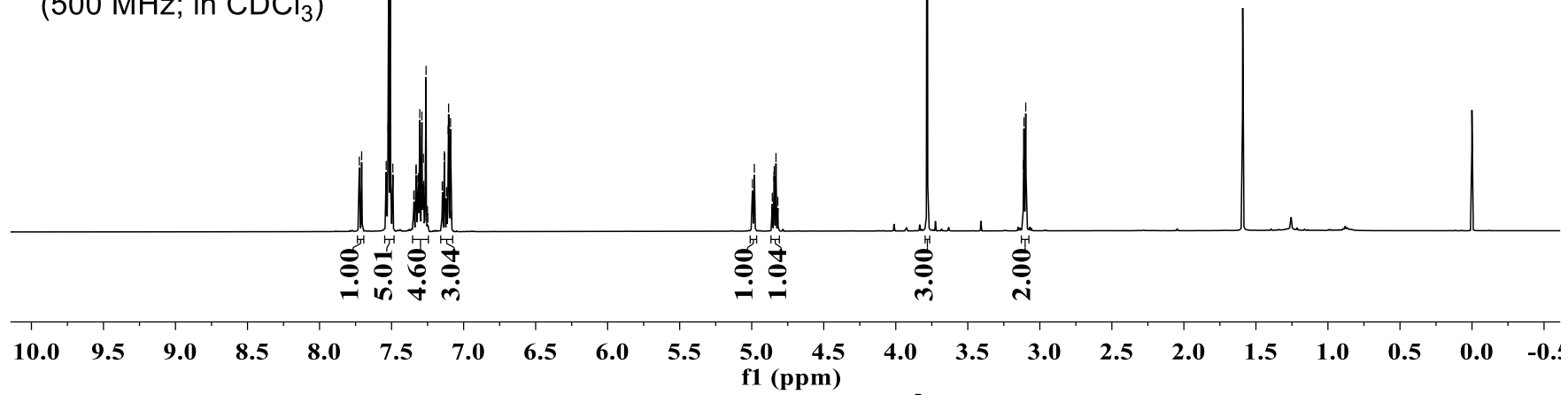
หน 药<smiles>COC(=O)/C(=C1/C[C@H]2[C@H]1c1ccccc1N2S(=O)(=O)c1ccc(Br)cc1)c1ccccc1</smiles>

(Z)-3da

(125 MHz; in $\mathrm{CDCl}_{3}$ )

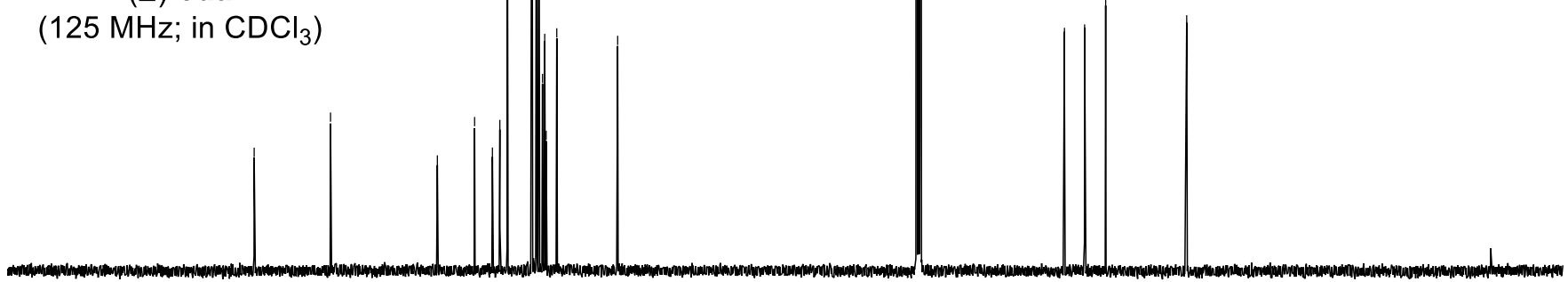


<smiles>COC(=O)/C(=C1/C[C@@H]2[C@@H]1[C@@H]1[C@@H]2c2ccccc2N1S(=O)(=O)c1ccc(Br)cc1)c1ccccc1</smiles>

$(E)-3$ da

$\left(500 \mathrm{MHz}\right.$; in $\mathrm{CDCl}_{3}$ )

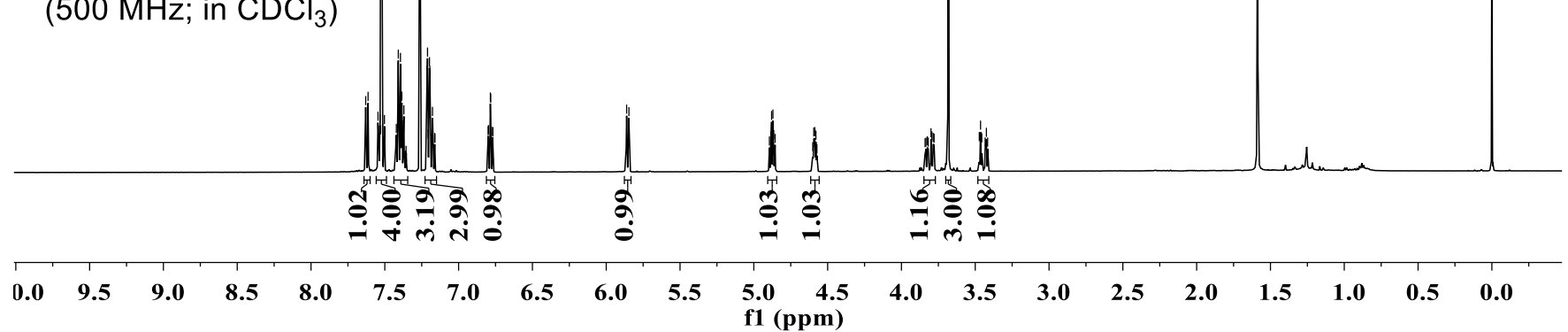

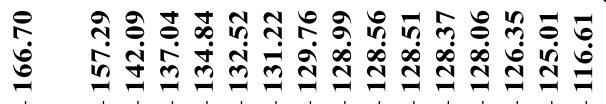<smiles>CC(=O)C(=C1C[C@@H]2[C@H]1c1ccccc1N2S(=O)(=O)c1ccc(Br)cc1)c1ccccc1</smiles>

(E)-3da

(125 MHz; in $\mathrm{CDCl}_{3}$ )

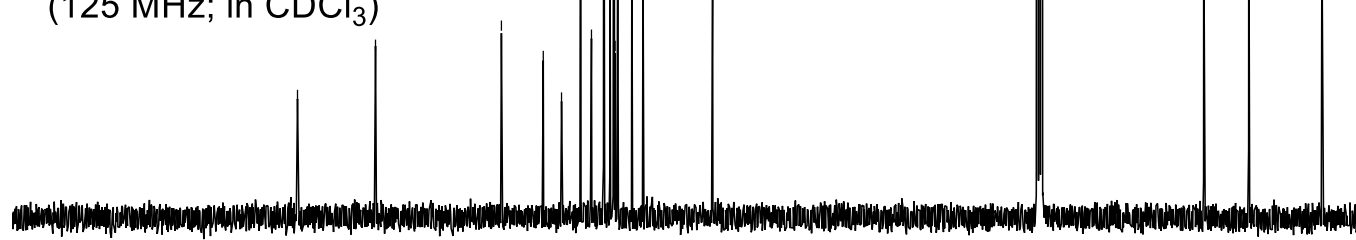




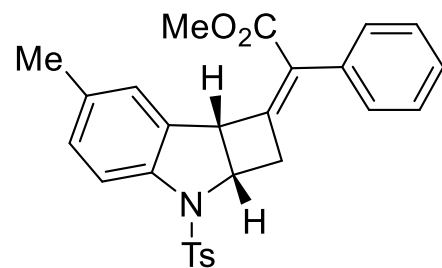

(Z)-3ea

$\left(500 \mathrm{MHz}\right.$; in $\left.\mathrm{CDCl}_{3}\right)$

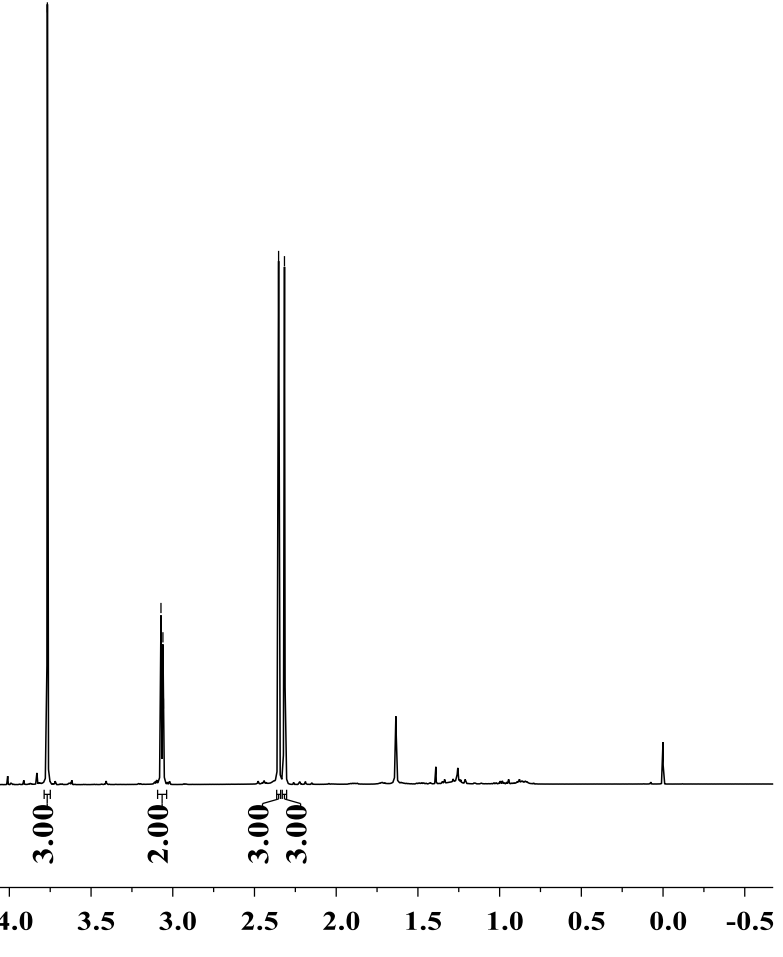

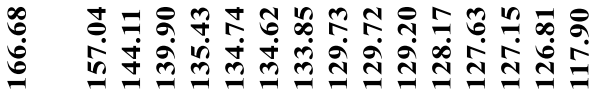

in

in

กิำ

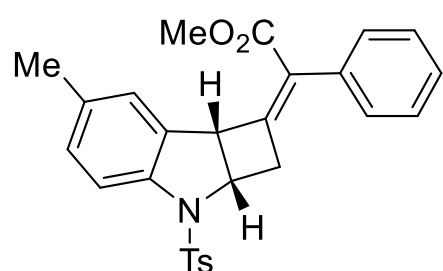

(Z)-3ea

(125 MHz; in $\mathrm{CDCl}_{3}$ ) 
<smiles>CC(=O)C1=C[C@H]2C[C@H]1c1cc(C)ccc1N2[13CH3]</smiles>

$(E)$-3ea

$\left(500 \mathrm{MHz}\right.$; in $\left.\mathrm{CDCl}_{3}\right)$

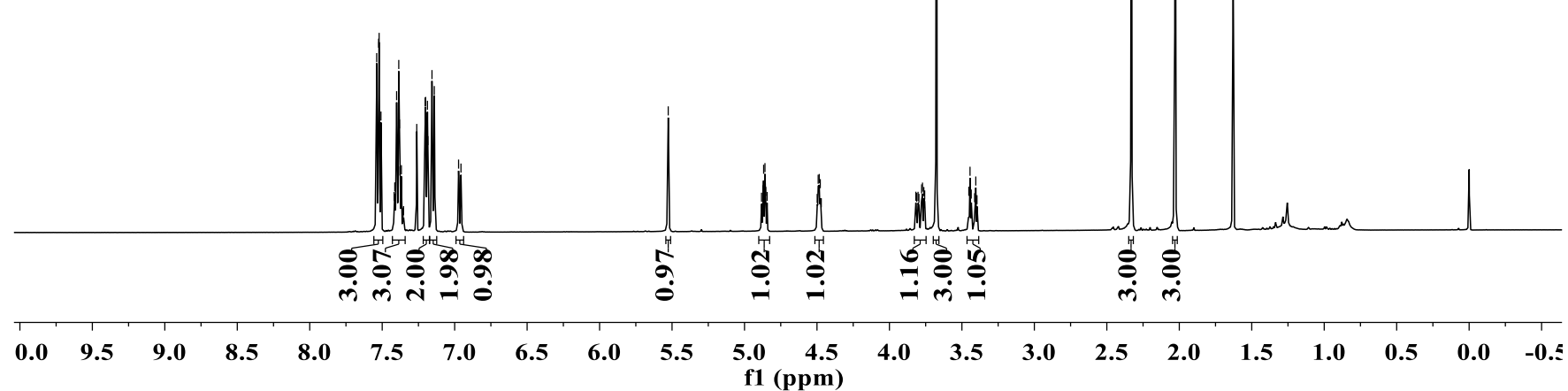

\section{능 \\ 空 \\ 華萑的

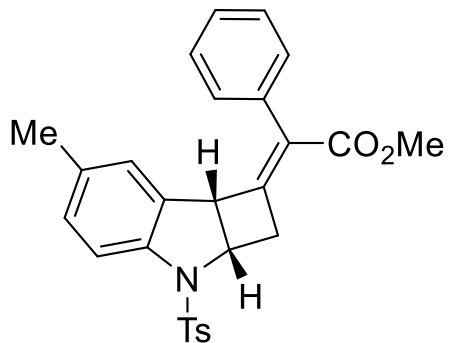

(E)-3ea

$\left(125 \mathrm{MHz}\right.$; in $\left.\mathrm{CDCl}_{3}\right)$ 


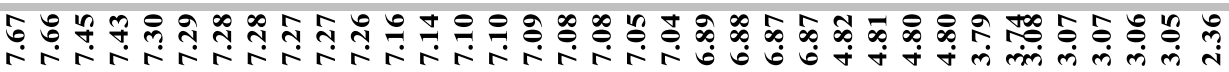

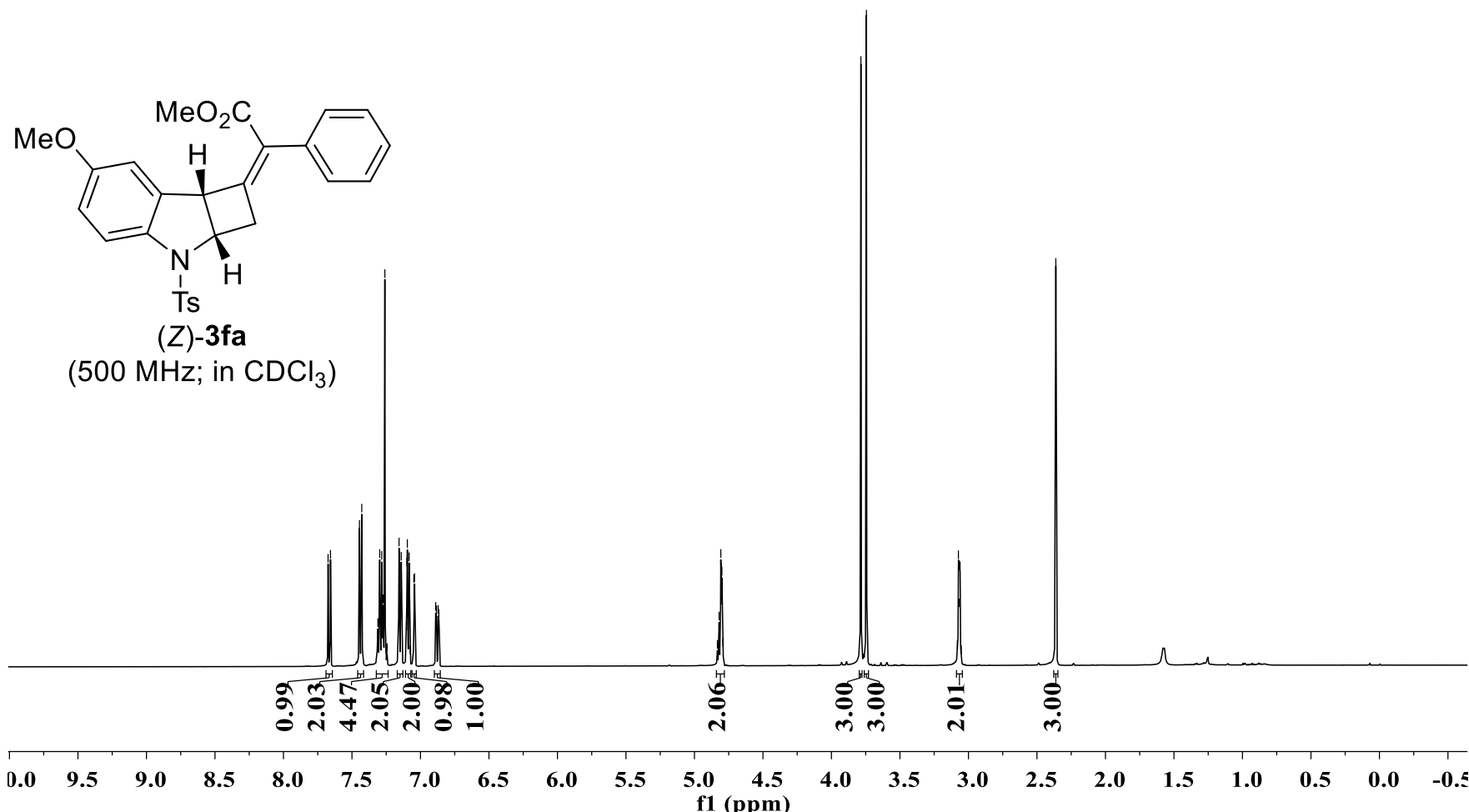

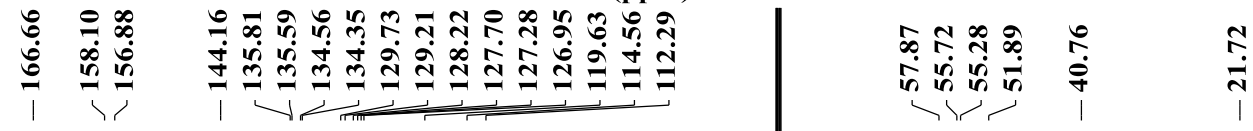

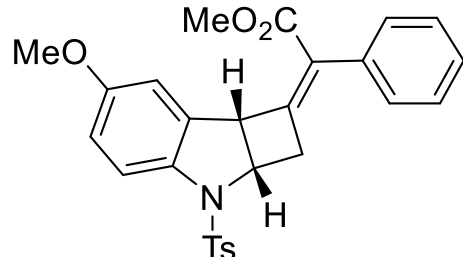

(Z)-3fa

$\left(125 \mathrm{MHz}\right.$; in $\left.\mathrm{CDCl}_{3}\right)$ 


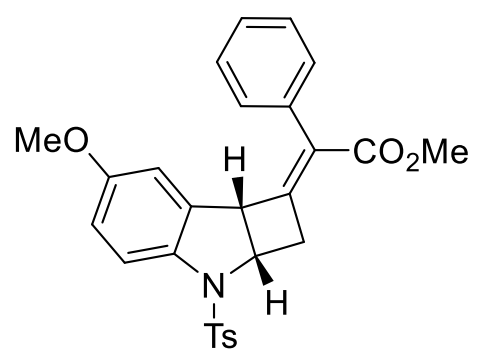

(E)-3fa

$\left(500 \mathrm{MHz}\right.$; in $\left.\mathrm{CDCl}_{3}\right)$

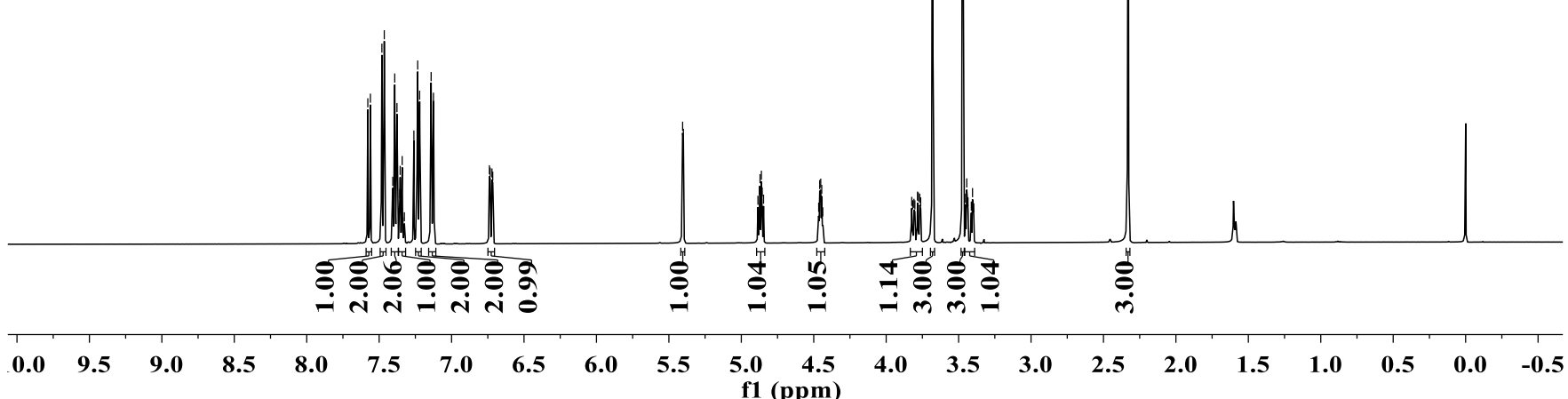

웅

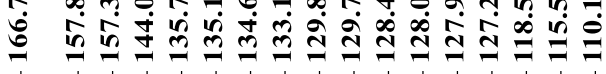

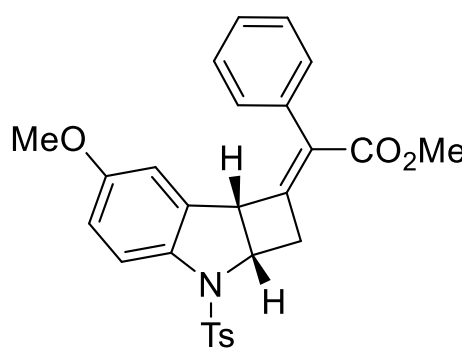

(E)-3fa

(125 $\mathrm{MHz}$; in $\mathrm{CDCl}_{3}$ )

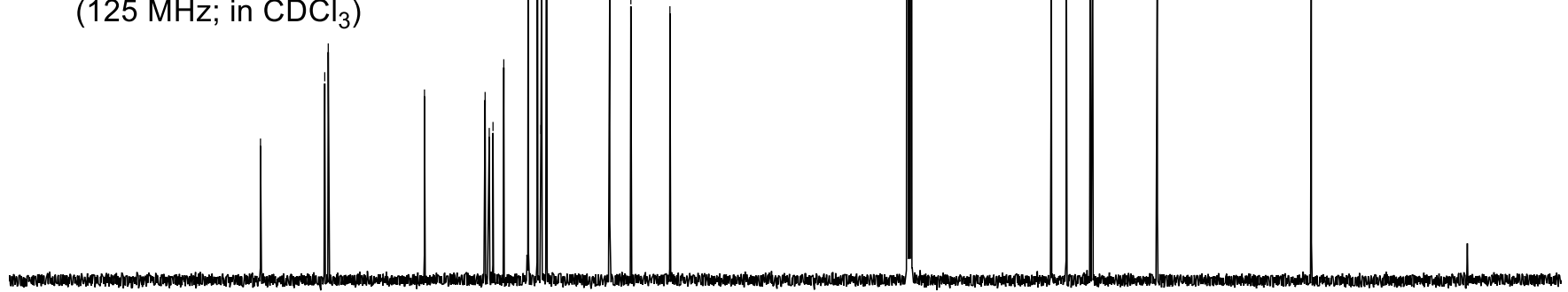




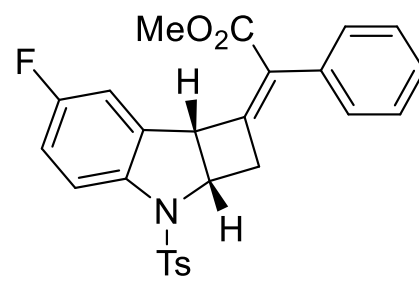

(Z)-3ga

$\left(500 \mathrm{MHz}\right.$; in $\left.\mathrm{CDCl}_{3}\right)$

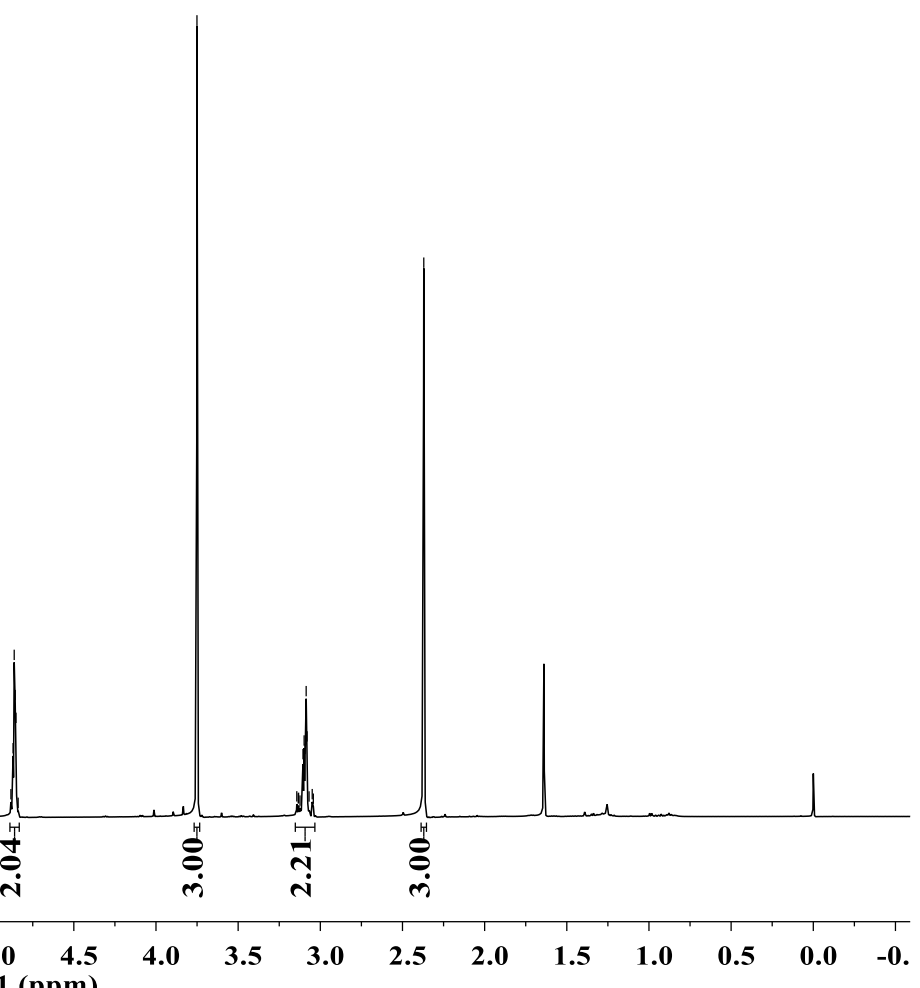

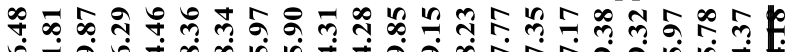

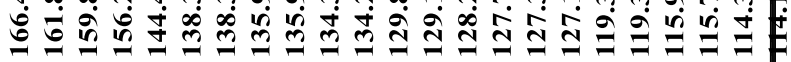

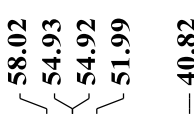

$\underset{\substack{+\infty}}{\infty}$

$\stackrel{\substack{\text { i } \\ \text { i }}}{2}$

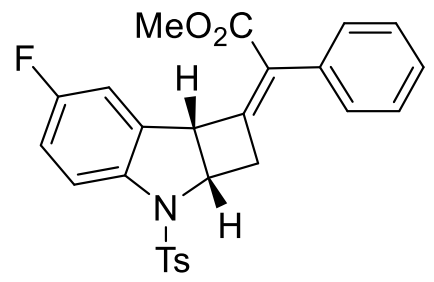

(Z)-3ga

$\left(125 \mathrm{MHz}\right.$; in $\mathrm{CDCl}_{3}$ )

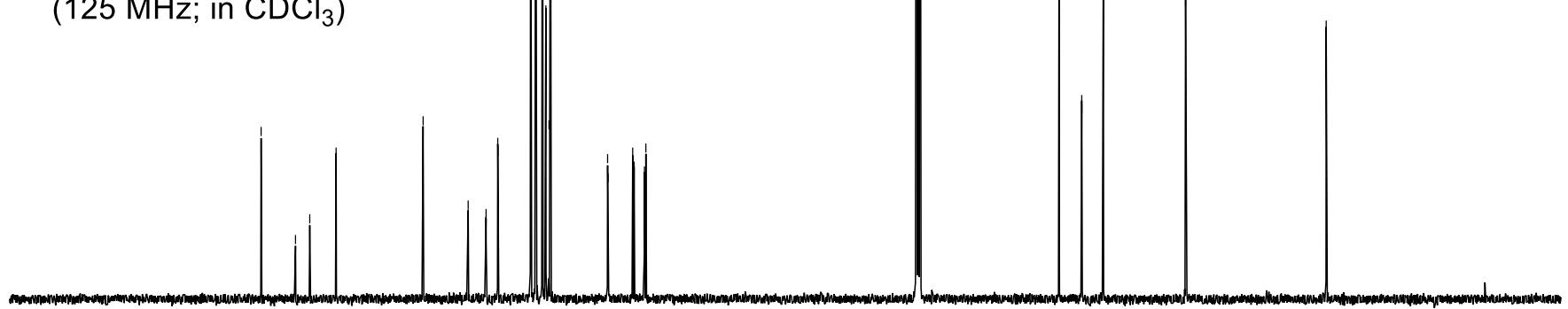




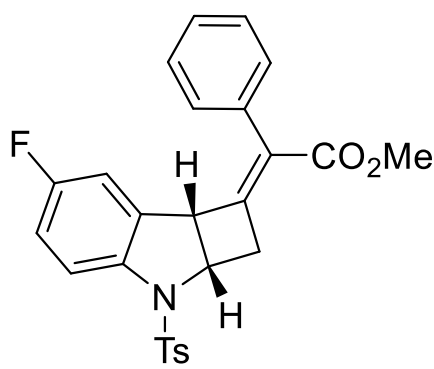

(E)-3ga

(500 MHz; in $\mathrm{CDCl}_{3}$ )

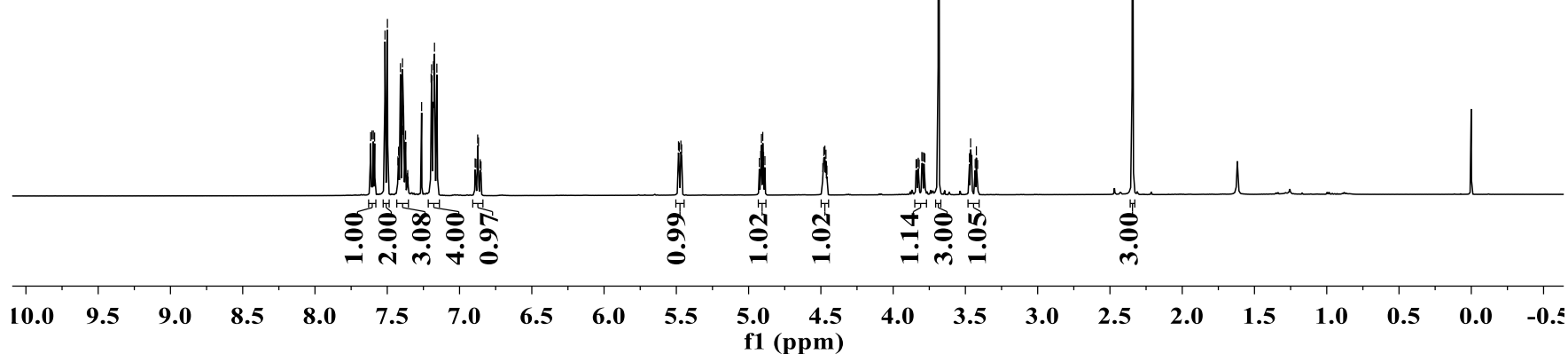

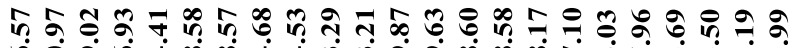

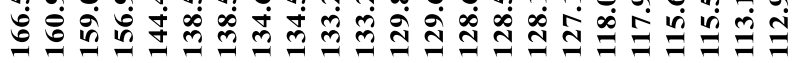<smiles></smiles>

(E)-3ga

$\left(125 \mathrm{MHz} ;\right.$ in $\left.\mathrm{CDCl}_{3}\right)$

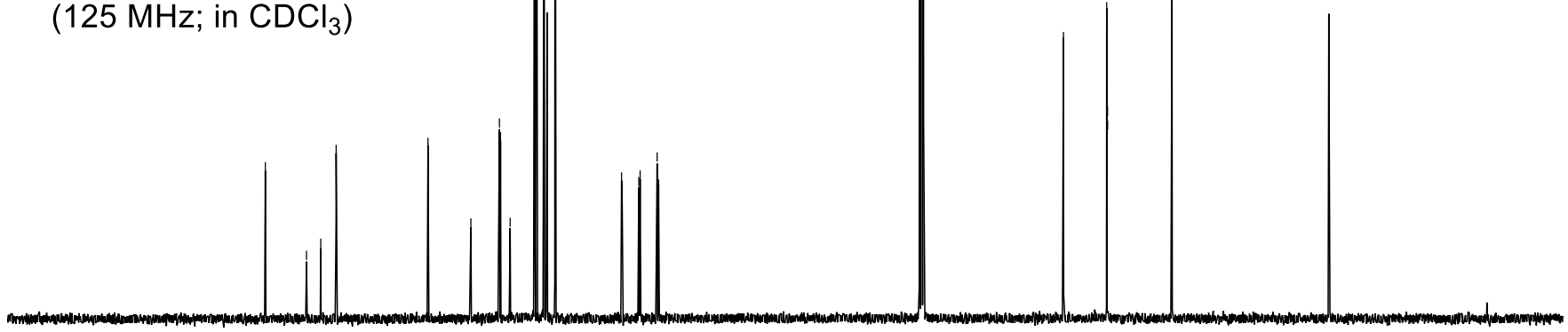




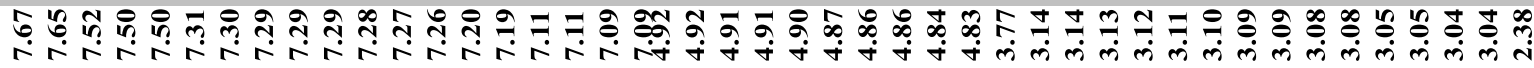

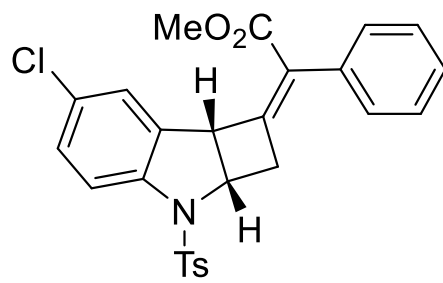

(Z)-3ha

$\left(500 \mathrm{MHz}\right.$; in $\mathrm{CDCl}_{3}$ )

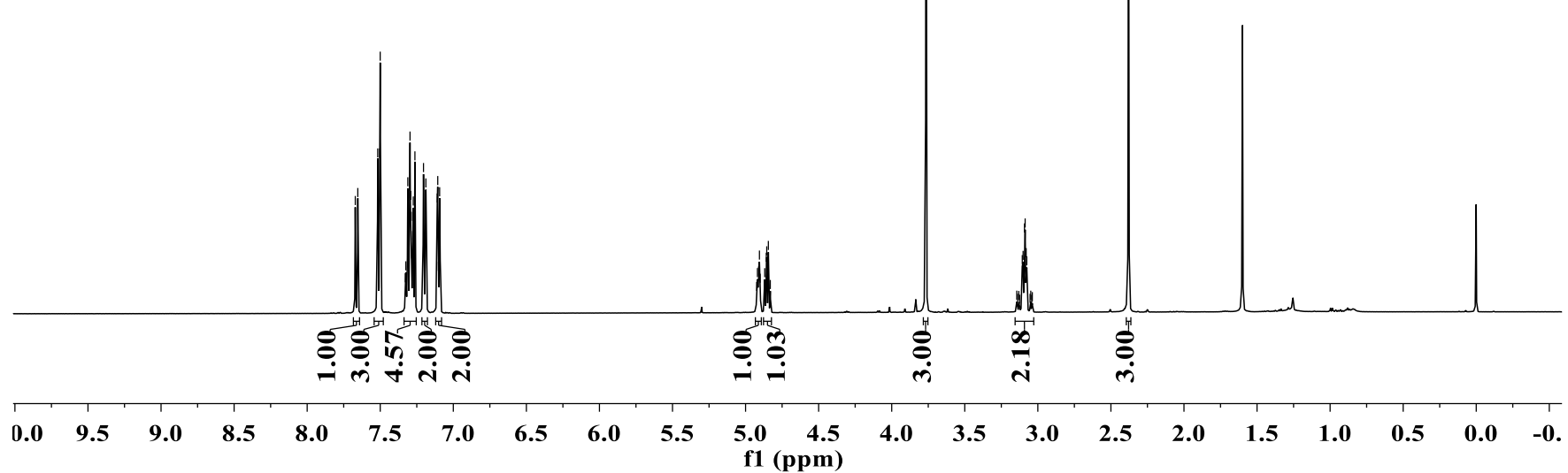

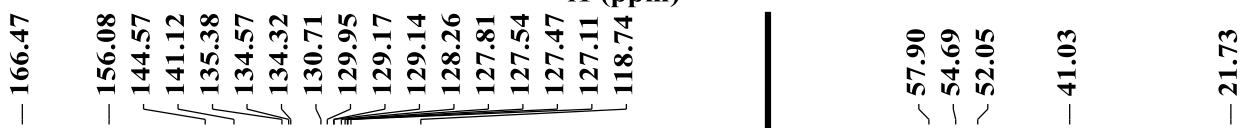

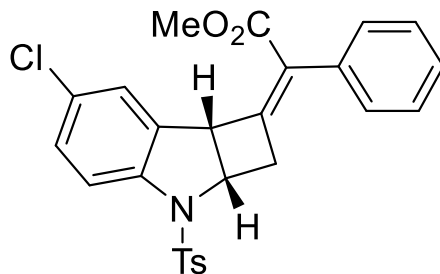

(Z)-3ha

$\left(125 \mathrm{MHz}\right.$; in $\mathrm{CDCl}_{3}$ ) 
<smiles>CC(=O)C1=C[C@H]2C[C@H]1c1cc(Cl)ccc1N2[13CH3]</smiles>

(E)-3ha

$\left(500 \mathrm{MHz}\right.$; in $\mathrm{CDCl}_{3}$ )

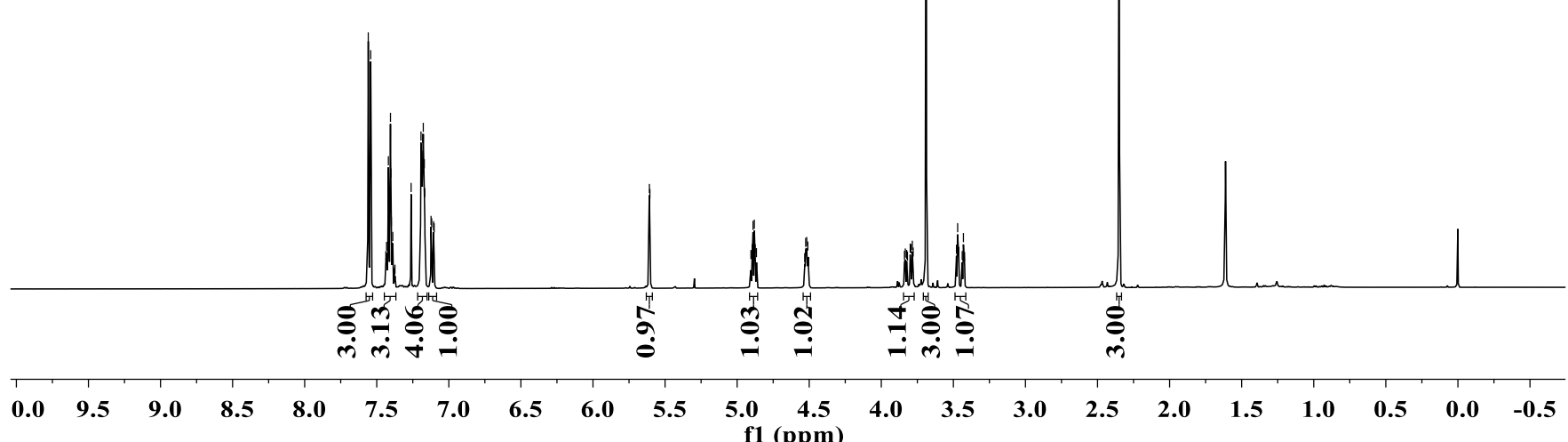

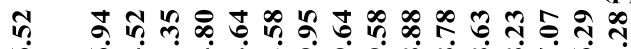

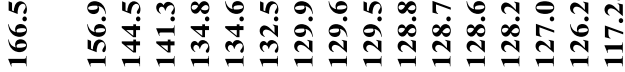<smiles>[3H]N1c2ccc(Cl)cc2[C@@H]2[C@@H]3C/C(=C(/C(C)=O)c4ccccc4)[C@H]3[C@H]21</smiles>

(E)-3ha

$\left(125 \mathrm{MHz}\right.$; in $\mathrm{CDCl}_{3}$ )

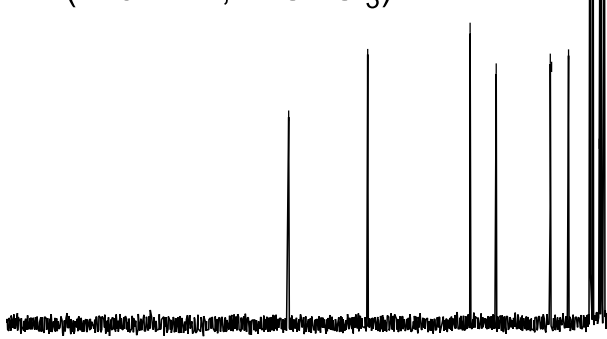




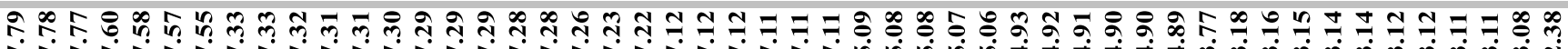

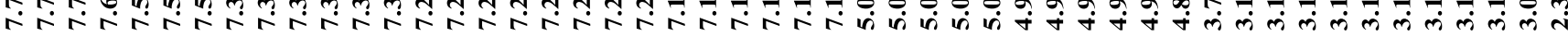

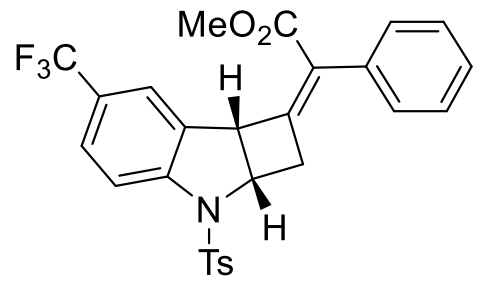

(Z)-3ia

$\left(500 \mathrm{MHz}\right.$; in $\left.\mathrm{CDCl}_{3}\right)$

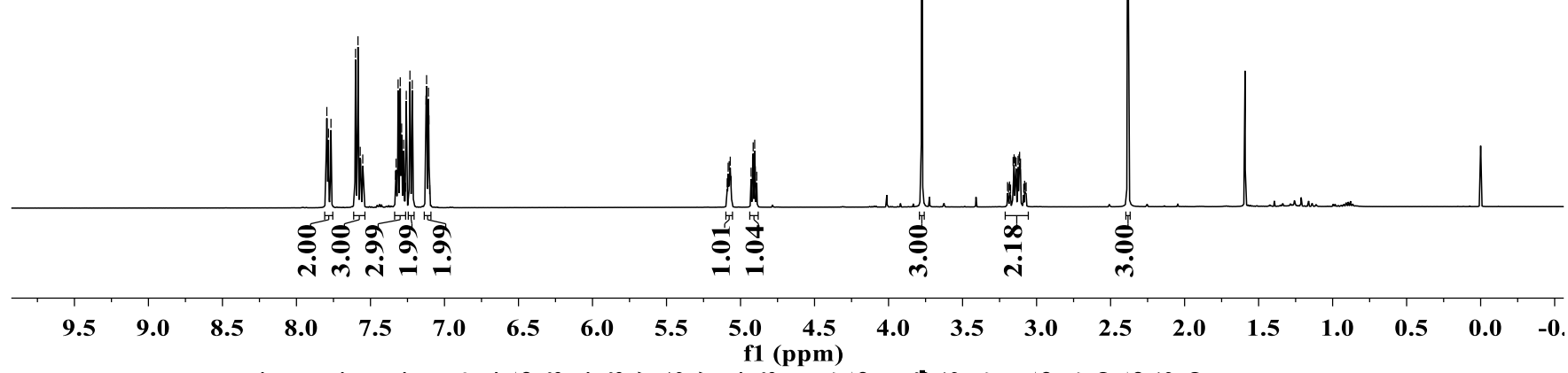

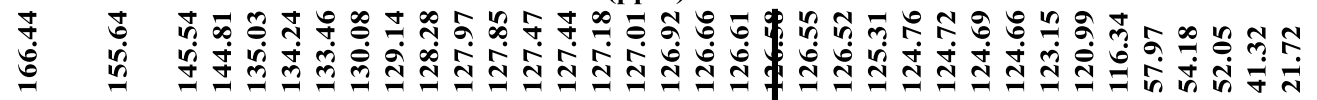<smiles>COC(=O)/C(=C1/C[C@H]2[C@H]1c1cc(C(F)(F)F)ccc1N2[14CH3])c1ccccc1</smiles>

(Z)-3ia

$\left(125 \mathrm{MHz}\right.$; in $\mathrm{CDCl}_{3}$ ) 


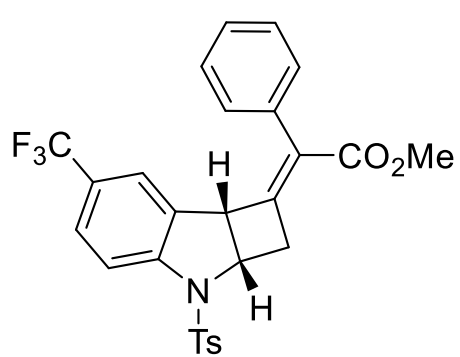

(E)-3ia

$\left(500 \mathrm{MHz}\right.$; in $\left.\mathrm{CDCl}_{3}\right)$

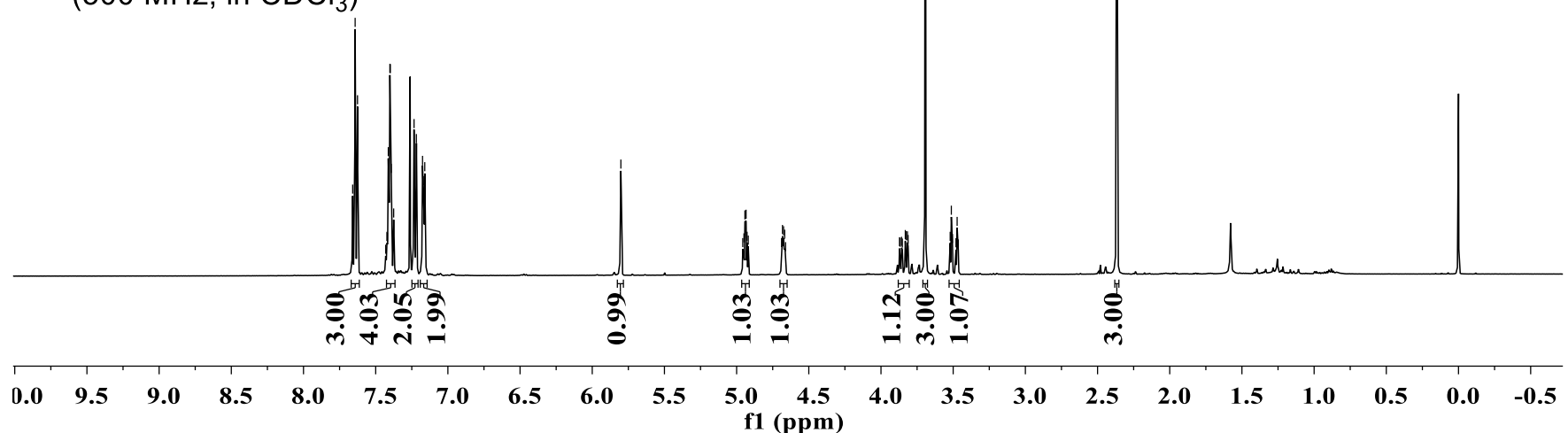

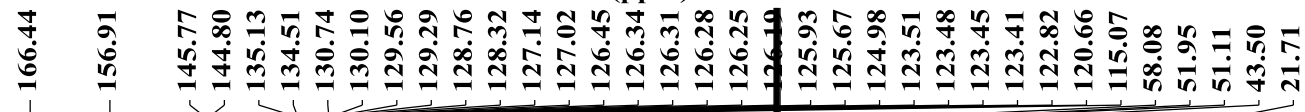

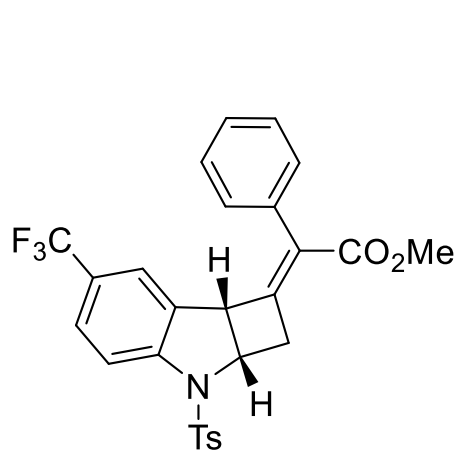

(E)-3ia

(125 MHz; in $\mathrm{CDCl}_{3}$ )

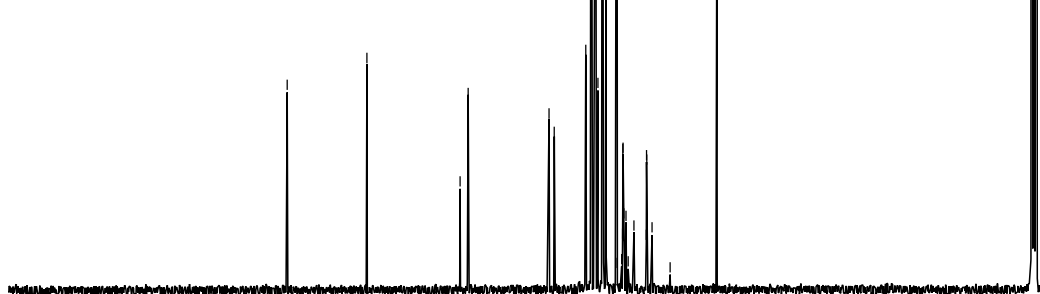


<smiles>[3H]N1c2cc(C)ccc2[C@H]2C(=C(OC)c3ccccc3)C[C@@H]21</smiles>

(Z)-3ja

$\left(500 \mathrm{MHz}\right.$; in $\mathrm{CDCl}_{3}$ )

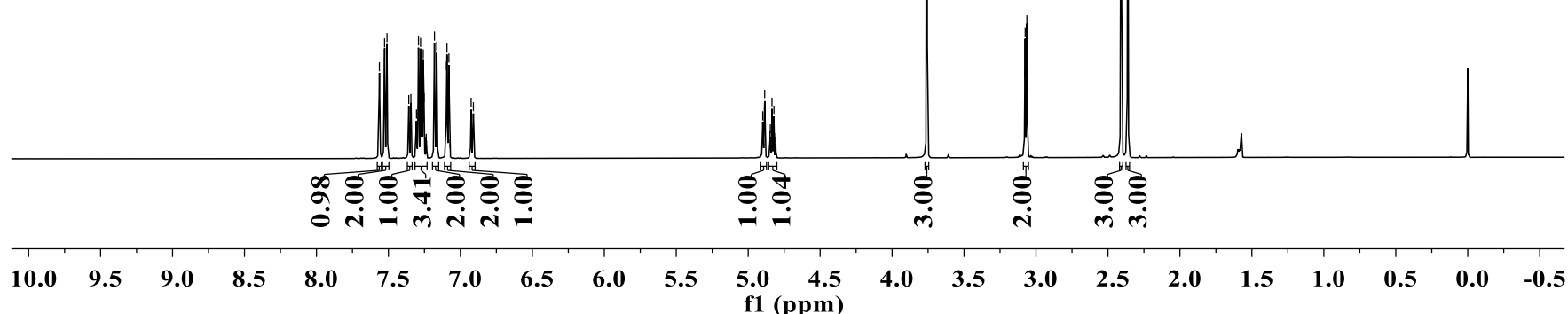

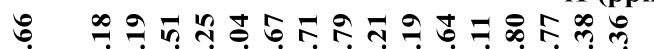

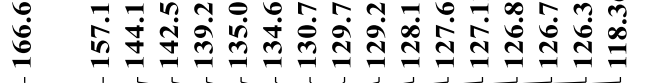<smiles>COC(=C1C[C@H]2[C@H]1c1ccc(C)cc1N2[13CH3])c1ccccc1</smiles>

(Z)-3ja (125 MHz; in $\mathrm{CDCl}_{3}$ )

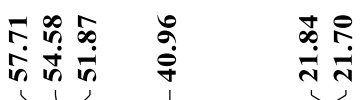




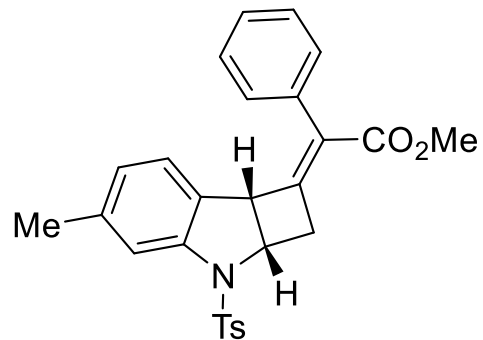

(E)-3ja

$\left(500 \mathrm{MHz}\right.$; in $\left.\mathrm{CDCl}_{3}\right)$

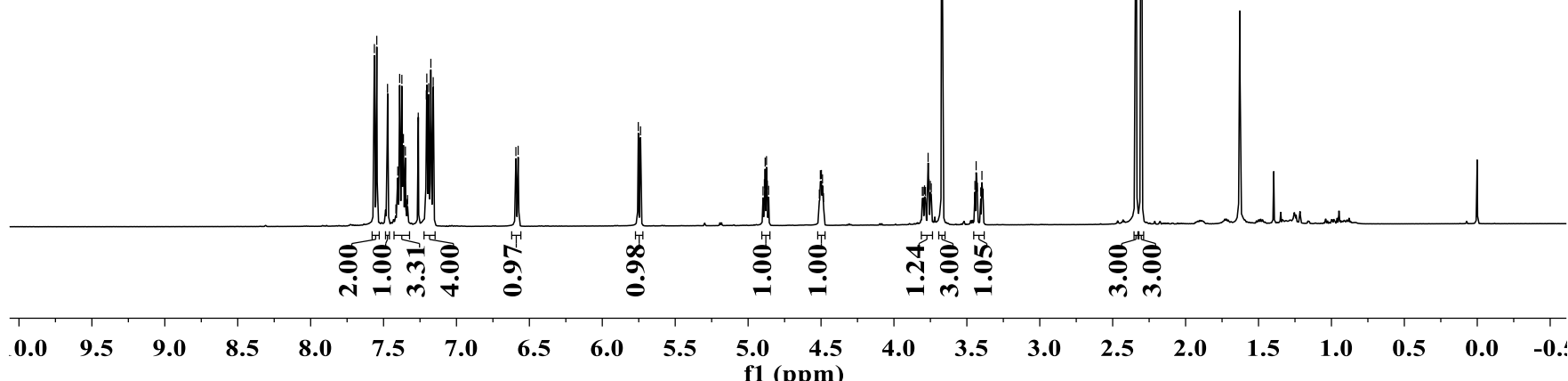

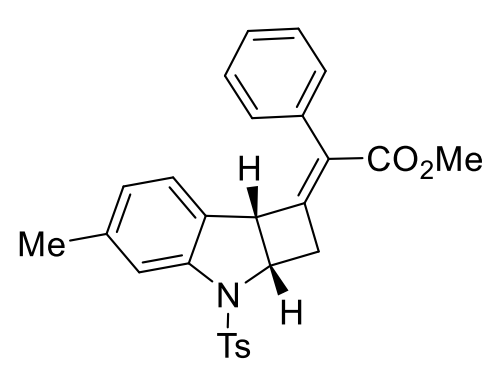

(E)-3ja 


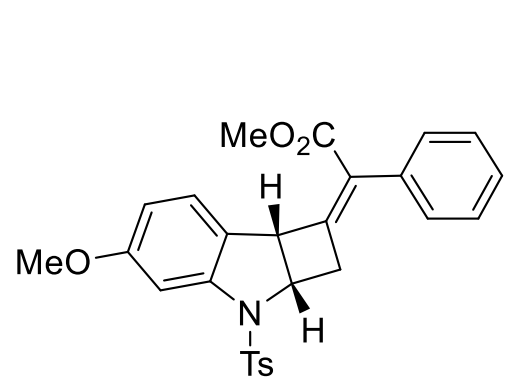

(Z)-3ka

$\left(500 \mathrm{MHz}\right.$; in $\mathrm{CDCl}_{3}$ )

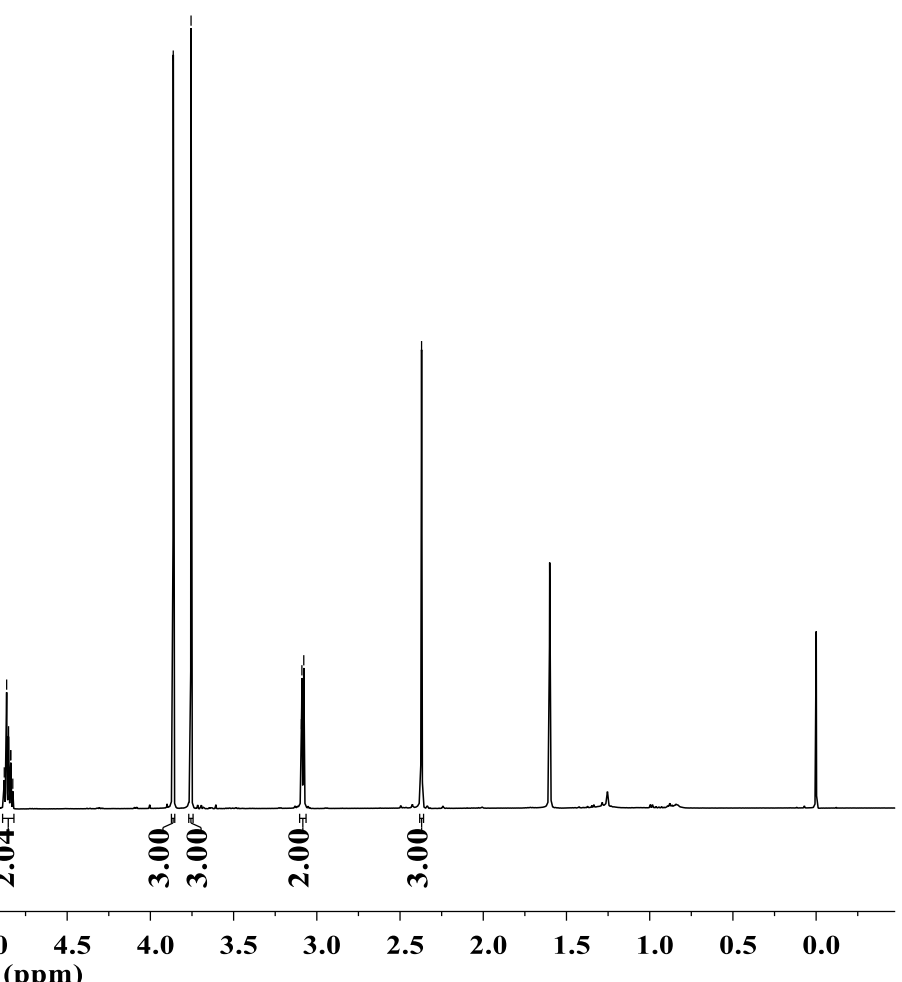

$\begin{array}{llllllllllll}0.0 & 9.5 & 9.0 & 8.5 & 8.0 & 7.5 & 7.0 & 6.5 & 6.0 & 5.5 & \begin{array}{l}5.0 \\ \text { f1 (ppm) }\end{array}\end{array}$

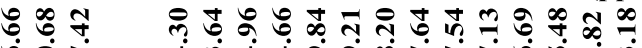

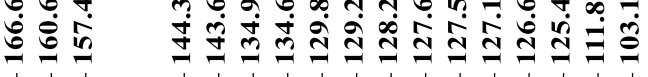<smiles>[3H]N1c2cc(OC)ccc2[C@H]2C(=C(OC)c3ccccc3)C[C@@H]21</smiles>

(Z)-3ka

(125 $\mathrm{MHz}$; in $\mathrm{CDCl}_{3}$ ) 


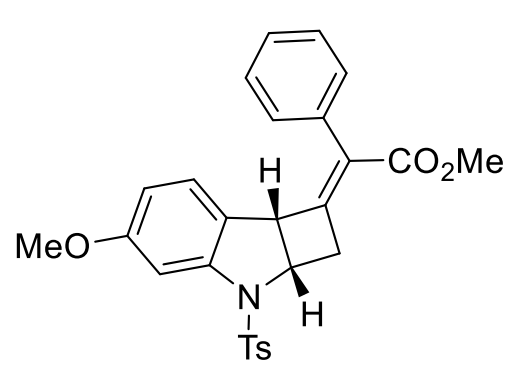

(E)-3ka

$\left(500 \mathrm{MHz}\right.$; in $\left.\mathrm{CDCl}_{3}\right)$

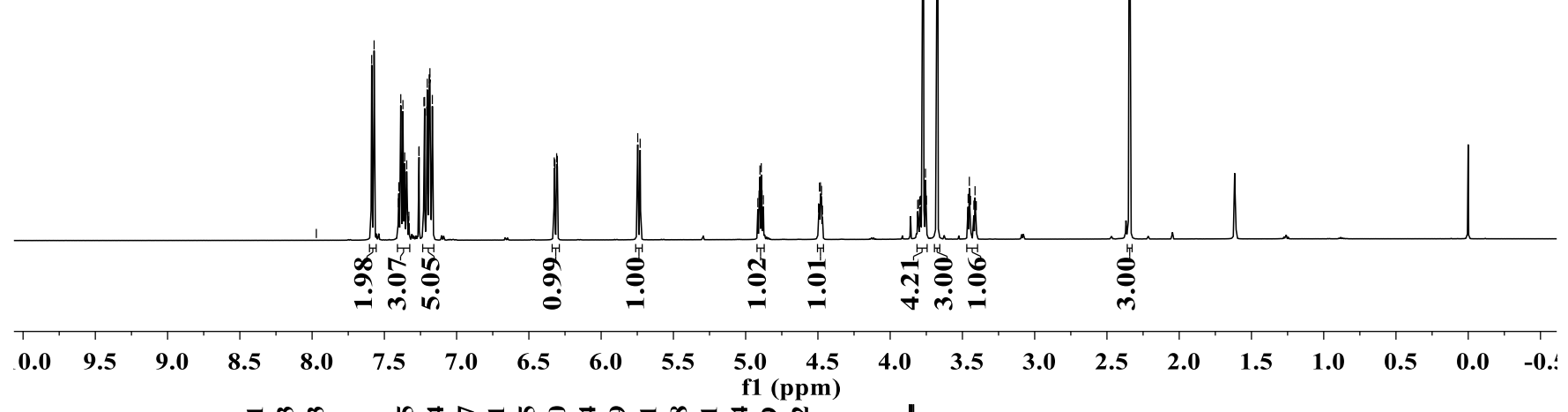

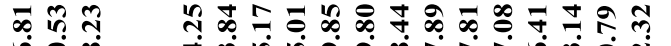

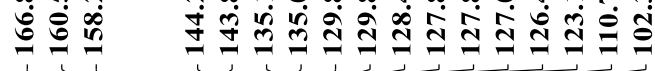

$1<1<1$

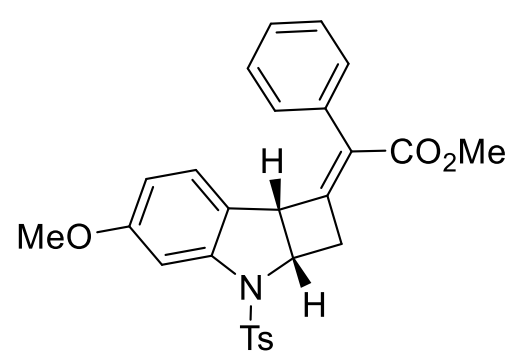

(E)-3ka

(125 MHz; in $\mathrm{CDCl}_{3}$ )

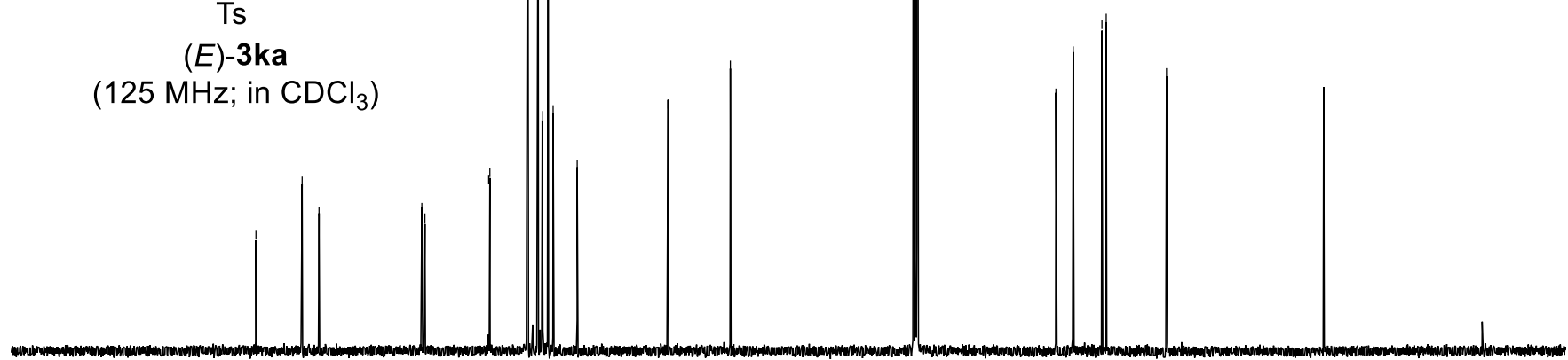

$\mathbf{0}$

190

180

$\begin{array}{lll}170 & 160 & 150\end{array}$

140

130

1201

$100 \quad 90$

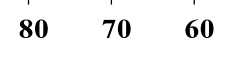

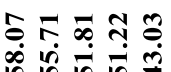

in 证的

$\underset{j}{i}$ 
<smiles>COC(=C1C[C@H]2[C@H]1c1ccc(F)cc1N2[13CH3])c1ccccc1</smiles>

(Z)-3la

$\left(500 \mathrm{MHz}\right.$; in $\left.\mathrm{CDCl}_{3}\right)$

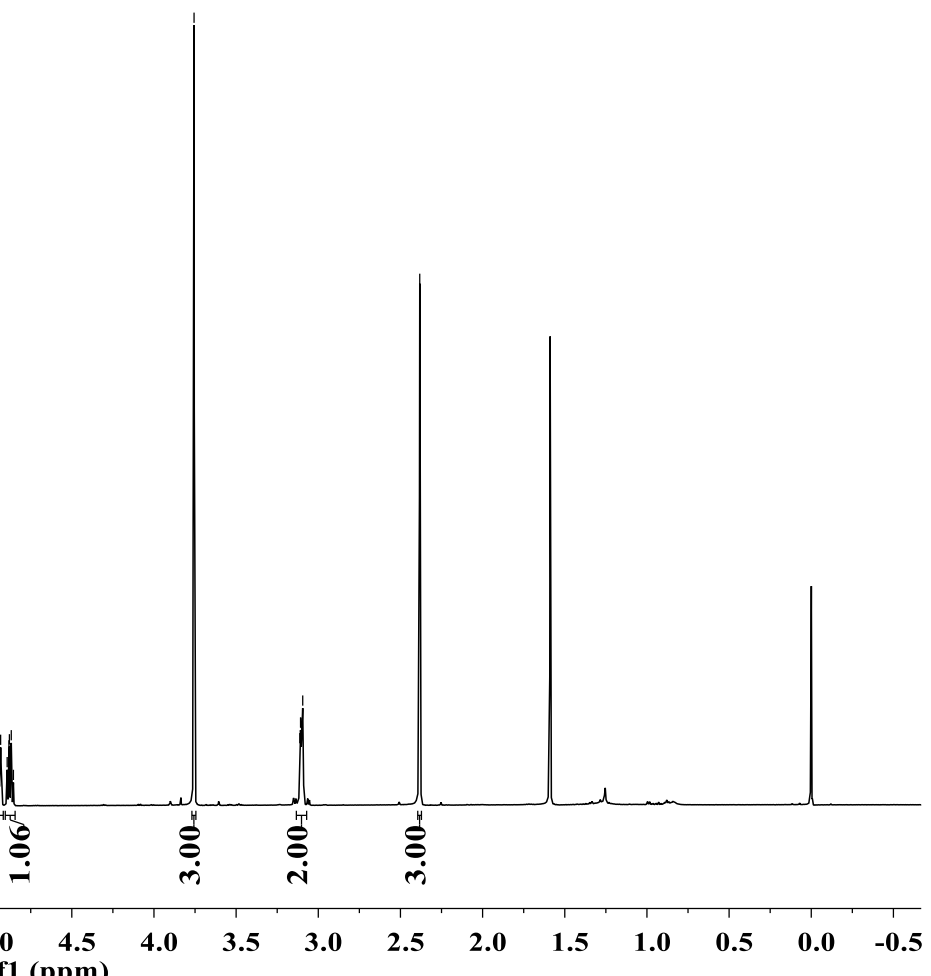

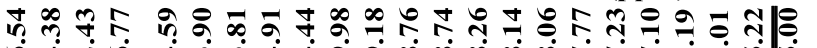

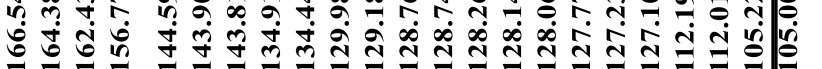

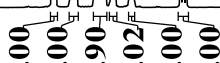
i

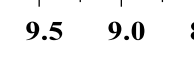

जा 7 , es

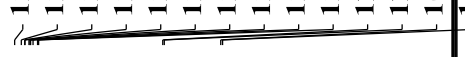

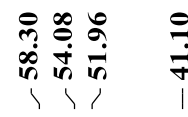

$\stackrel{m}{i}$

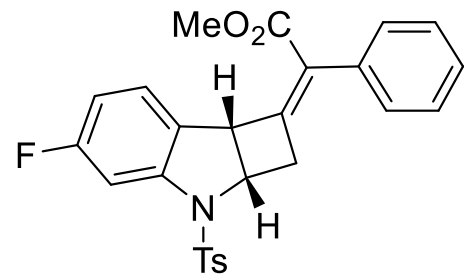

(Z)-3la

(125 MHz; in $\mathrm{CDCl}_{3}$ ) 
<smiles>COC(=O)/C(=C1\C[C@H]2[C@H]1c1ccc(F)cc1N2[AsH3])c1ccccc1</smiles>

(E)-3la

$\left(500 \mathrm{MHz}\right.$; in $\left.\mathrm{CDCl}_{3}\right)$

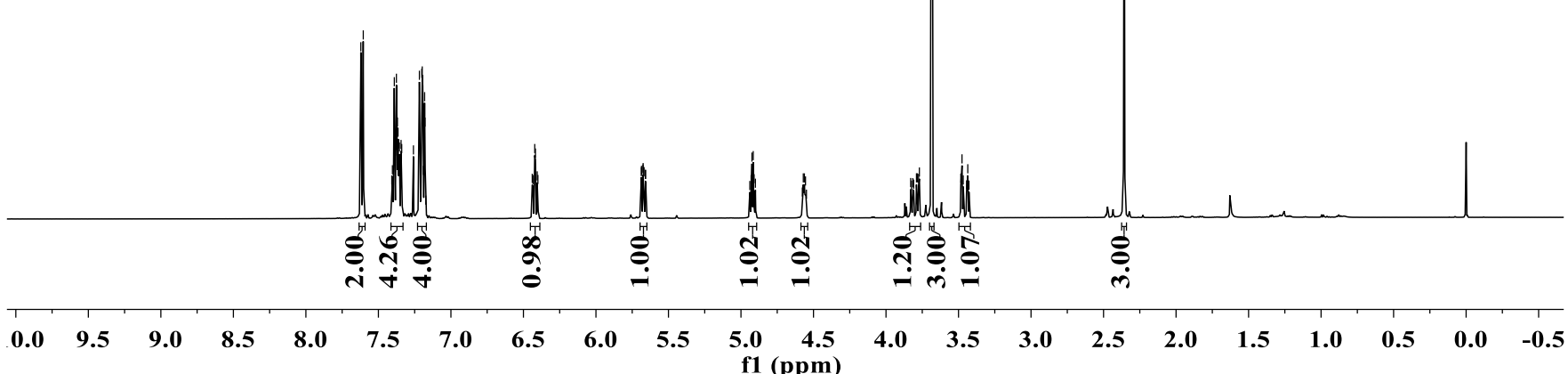

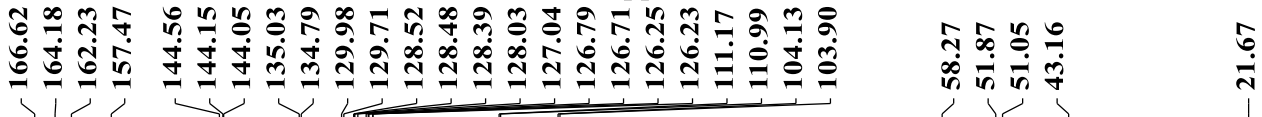

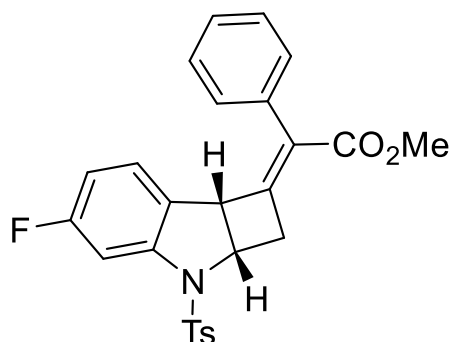

(E)-3la

$\left(125 \mathrm{MHz}\right.$; in $\mathrm{CDCl}_{3}$ )

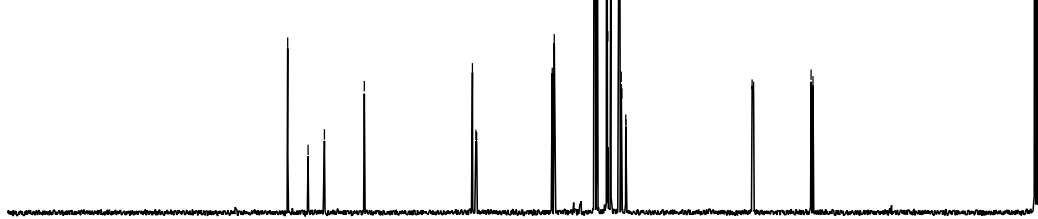

$\stackrel{\substack{0 \\ \hline}}{1}$

$\begin{array}{llllllllllllllllllllll}00 & 190 & 180 & 170 & 160 & 150 & 140 & 130 & 120 & 110 & 100 & 90 & 80 & 70 & 60 & 50 & 40 & 30 & 20 & 10 & 0 & \end{array}$ 

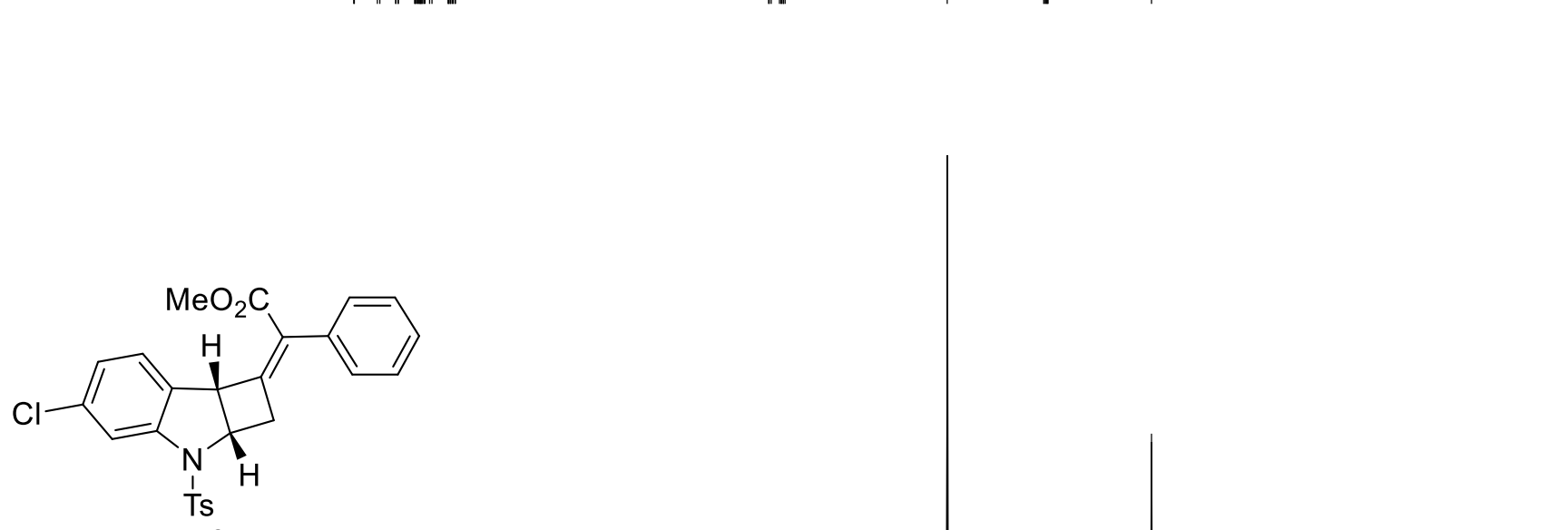

(Z)-3ma

$\left(500 \mathrm{MHz}\right.$; in $\left.\mathrm{CDCl}_{3}\right)$

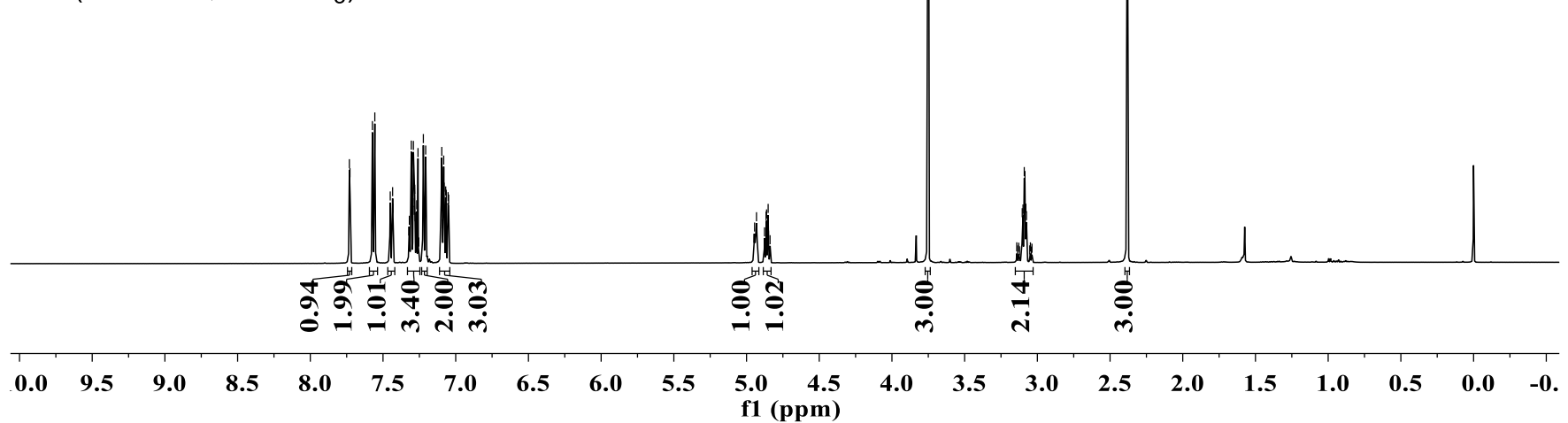

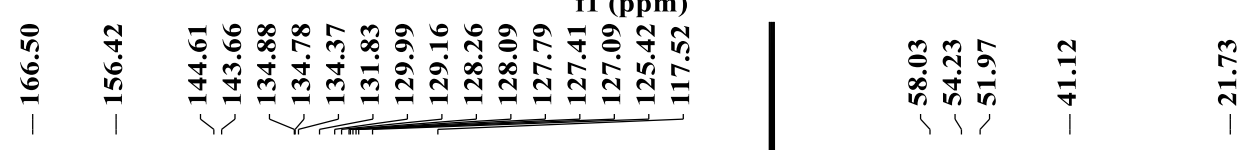

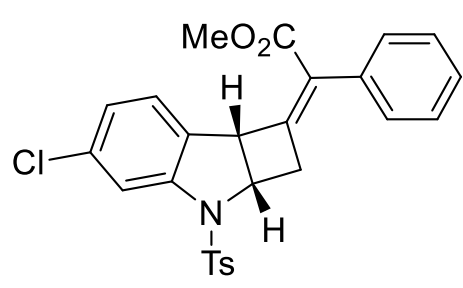

(Z)-3ma

$\left(125 \mathrm{MHz}\right.$; in $\left.\mathrm{CDCl}_{3}\right)$ 


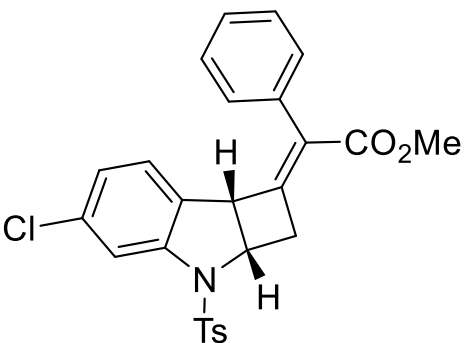

(E)-3ma

$\left(500 \mathrm{MHz}\right.$; in $\left.\mathrm{CDCl}_{3}\right)$

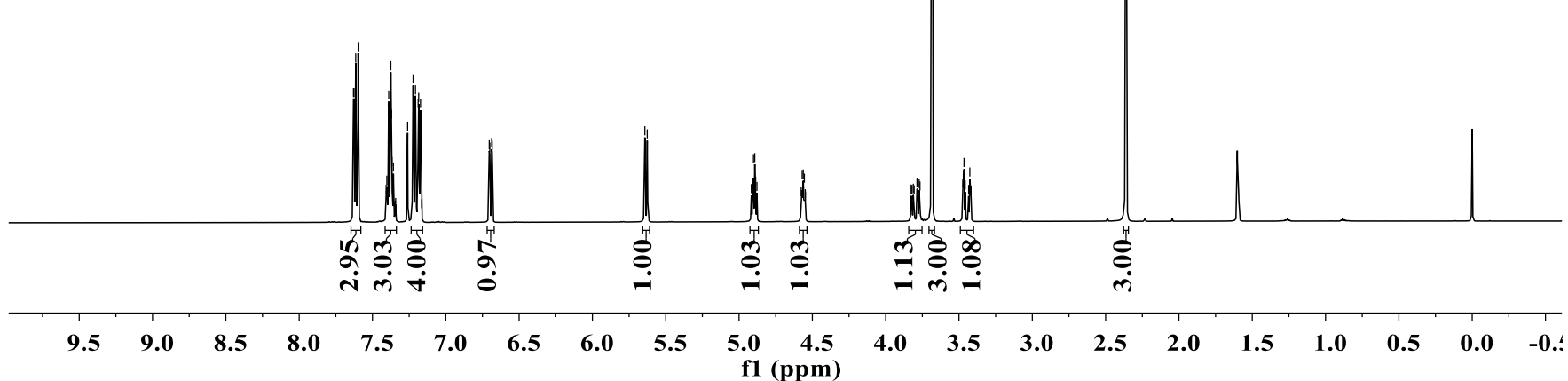

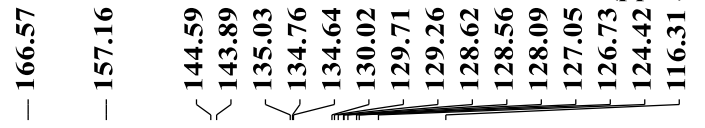

จุ 的的年 方<smiles>CC(=O)/C(=C1\C[C@H]2[C@H]1c1ccc(Cl)cc1N2[As])c1ccccc1</smiles>

(E)-3ma

(125 MHz; in $\mathrm{CDCl}_{3}$ )

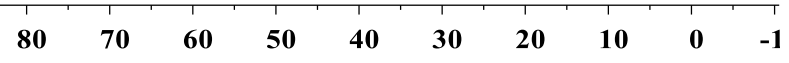


<smiles>COC/C(=C1/C[C@H]2[C@H]1c1c(C)cccc1N2[AsH3])c1ccccc1</smiles>

(Z)-3na

$\left(500 \mathrm{MHz}\right.$; in $\left.\mathrm{CDCl}_{3}\right)$

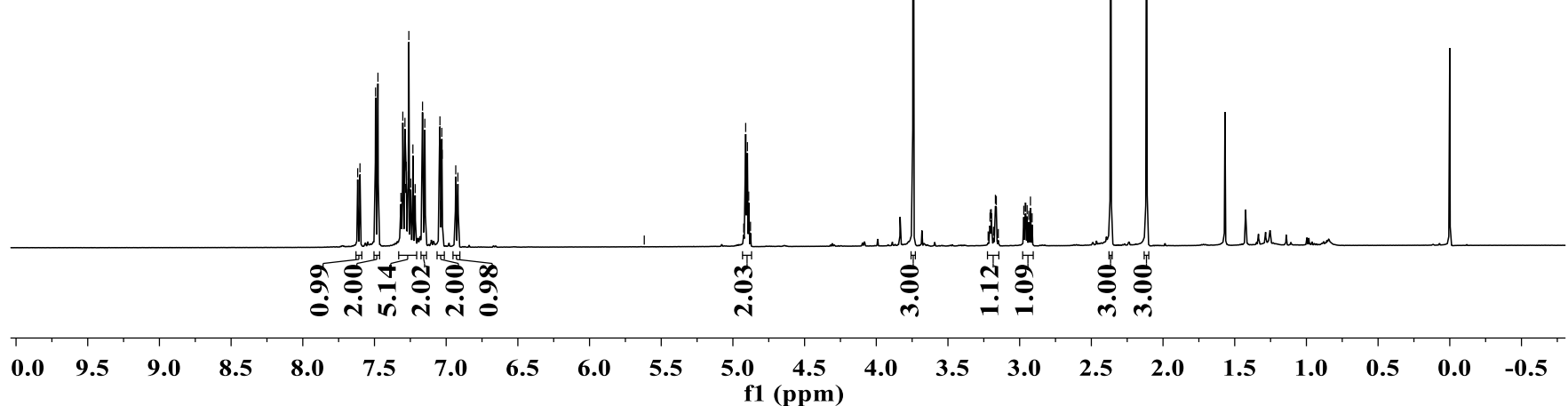

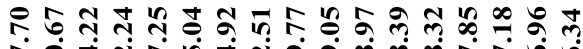

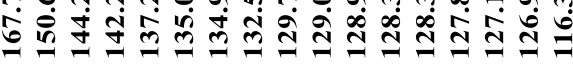

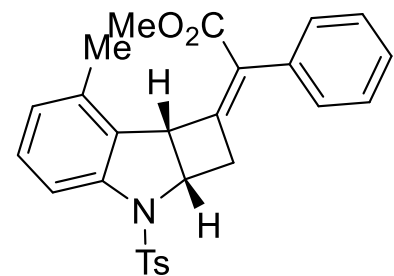

(Z)-3na

(125 $\mathrm{MHz}$; in $\mathrm{CDCl}_{3}$ ) 


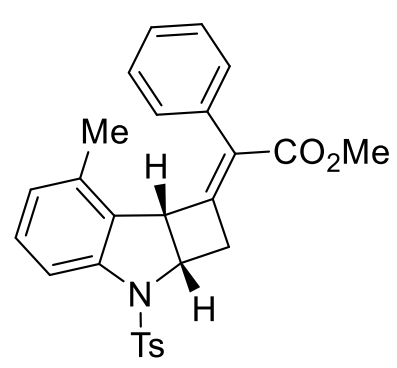

(E)-3na

$\left(500 \mathrm{MHz}\right.$; in $\left.\mathrm{CDCl}_{3}\right)$

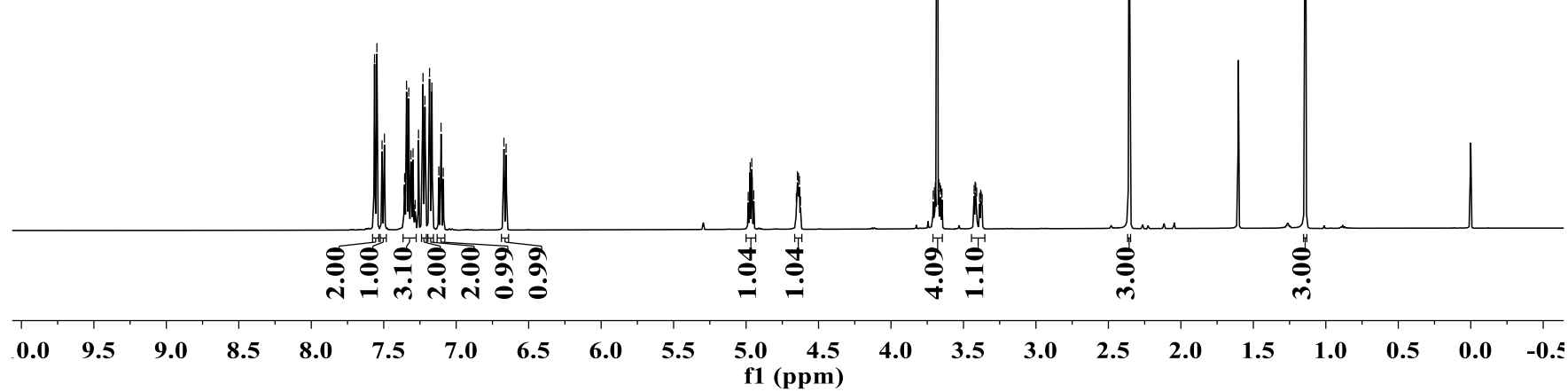

ஸे

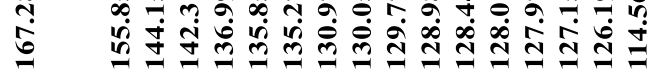

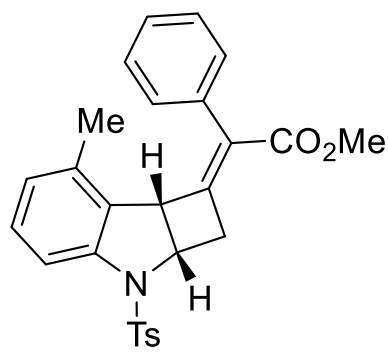

(E)-3na

$\left(125 \mathrm{MHz}\right.$; in $\mathrm{CDCl}_{3}$ ) 
<smiles>COC(=C1C[C@H]2[C@H]1c1cccc(C)c1N2[AsH3])c1ccccc1</smiles>

(Z)-3oa

$\left(500 \mathrm{MHz}\right.$; in $\left.\mathrm{CDCl}_{3}\right)$

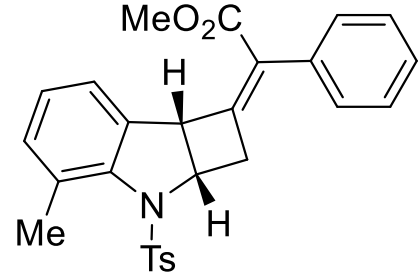

(Z)-3oa

$\left(125 \mathrm{MHz}\right.$; in $\mathrm{CDCl}_{3}$ )

*

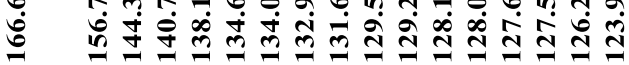

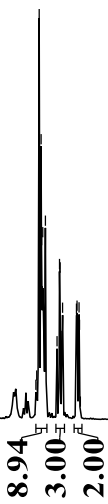

दे ठำ
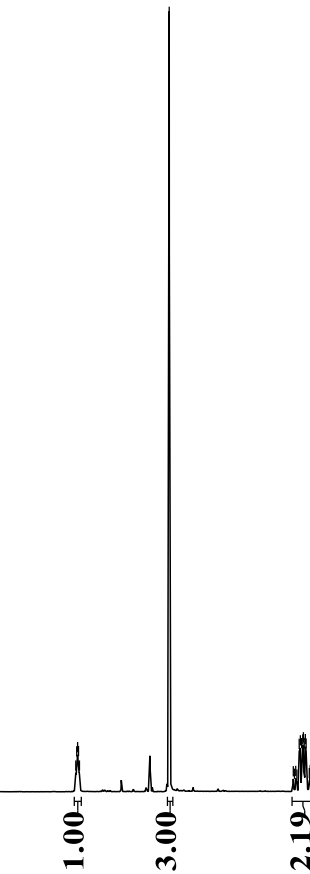

ते हैं
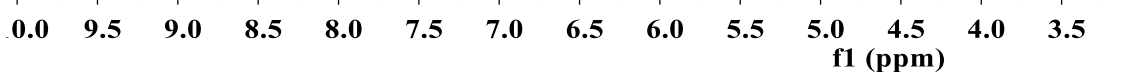

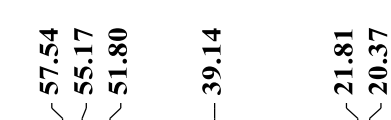

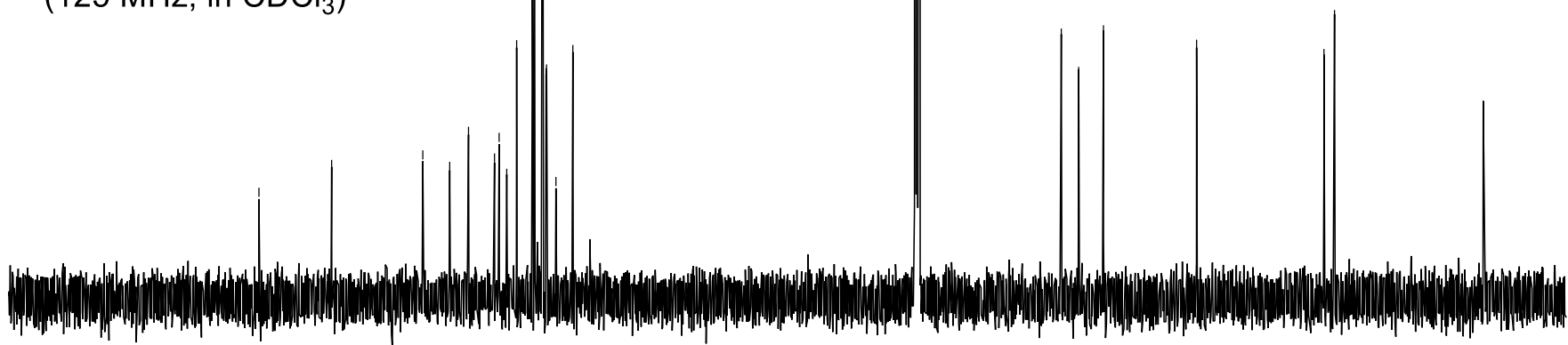




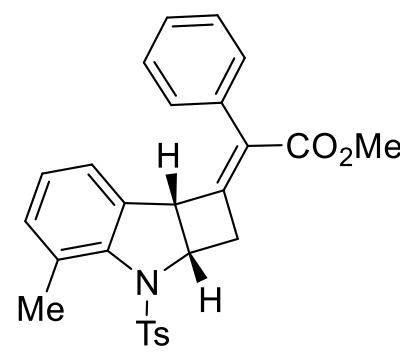

(E)-3oa

$\left(500 \mathrm{MHz} ;\right.$ in $\left.\mathrm{CDCl}_{3}\right)$

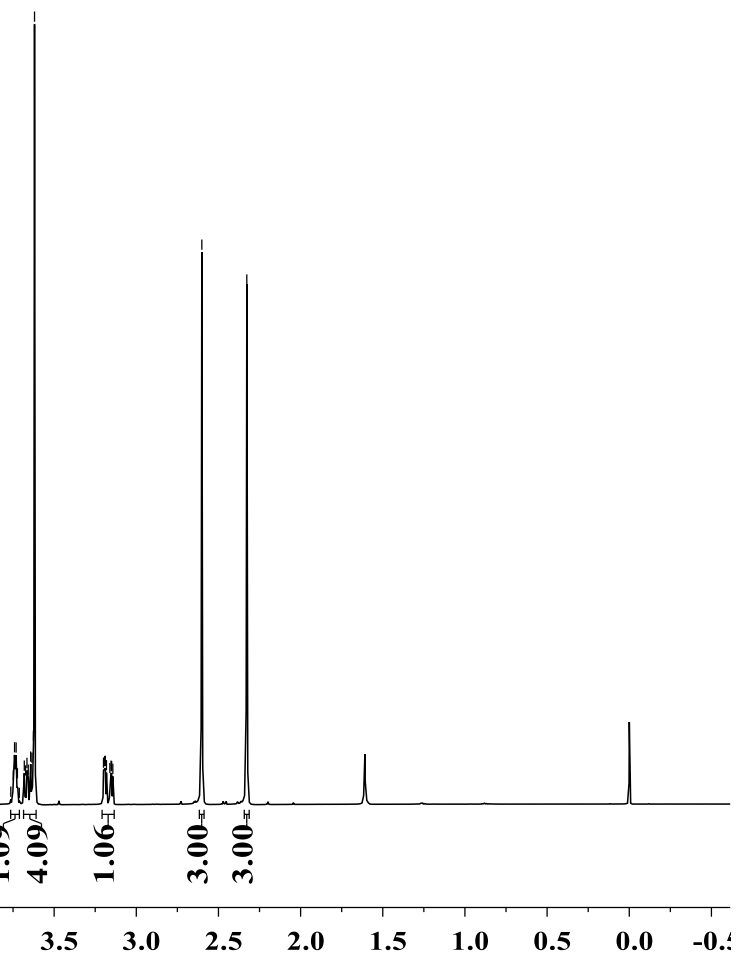

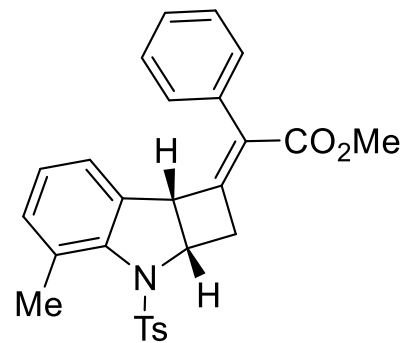

(E)-3oa

(125 MHz; in $\mathrm{CDCl}_{3}$ )

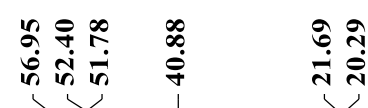




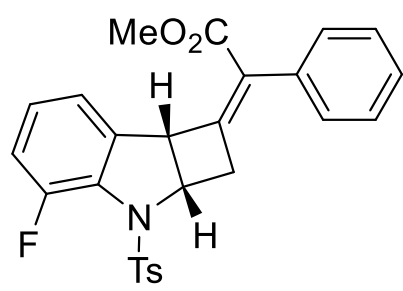

(Z)-3pa

$\left(500 \mathrm{MHz}\right.$; in $\mathrm{CDCl}_{3}$ )

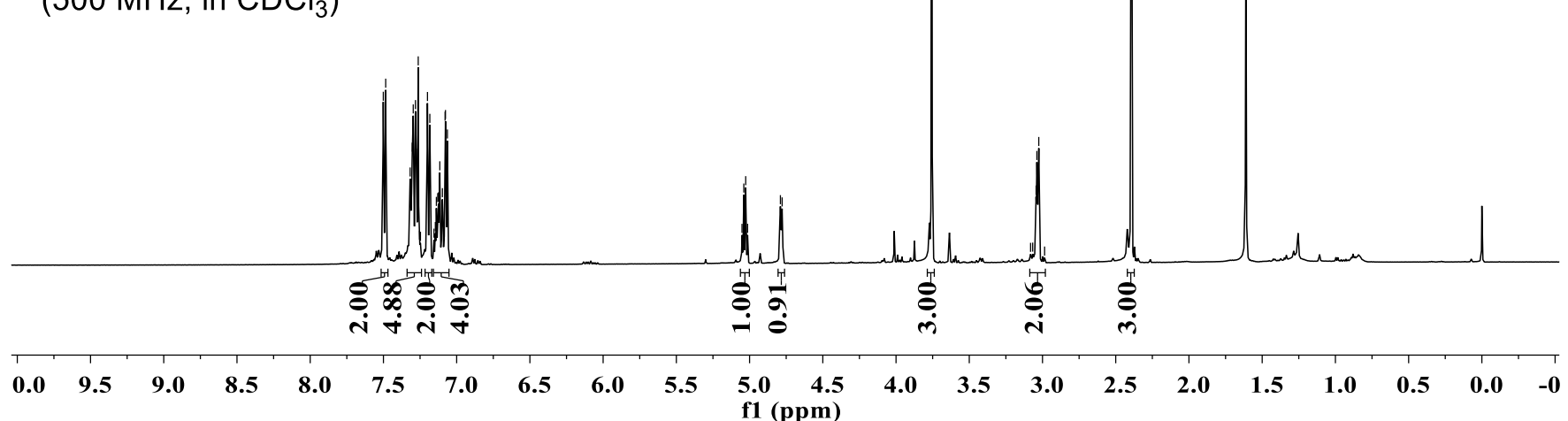

เก

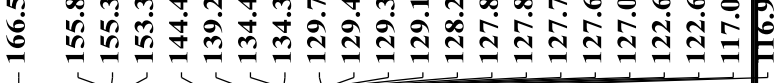

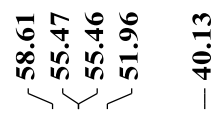

$\stackrel{\text { ก }}{i}$

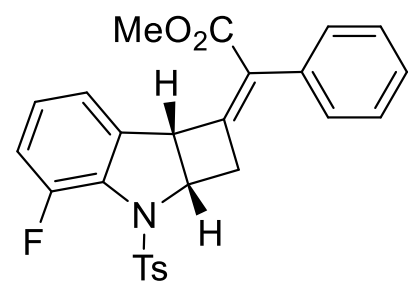

(Z)-3pa

$\left(125 \mathrm{MHz}\right.$; in $\left.\mathrm{CDCl}_{3}\right)$

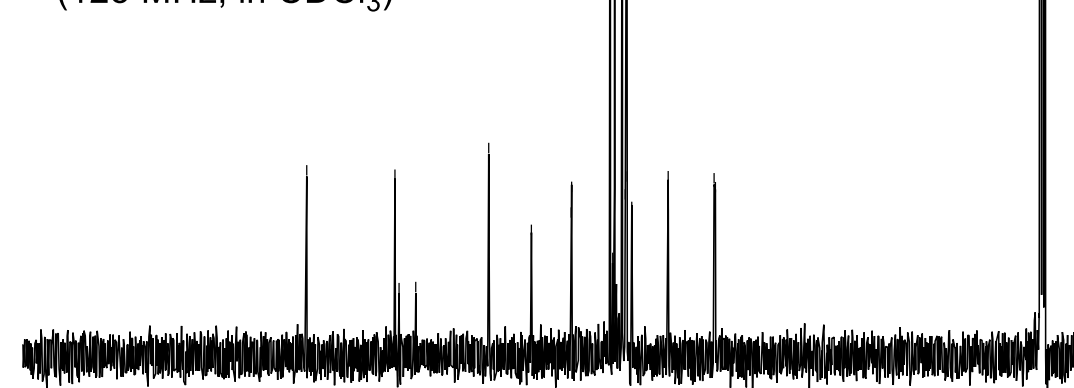




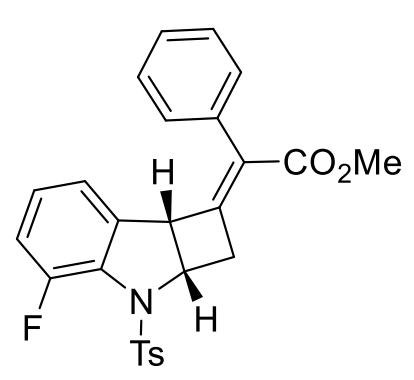

(E)-3pa

$\left(500 \mathrm{MHz}\right.$; in $\left.\mathrm{CDCl}_{3}\right)$

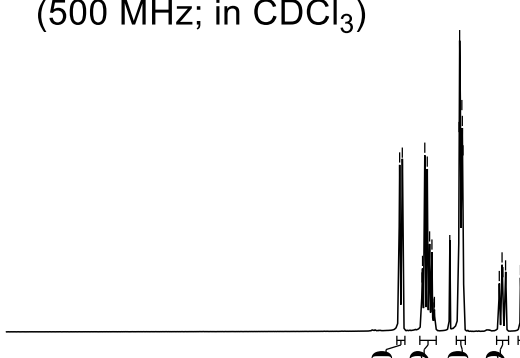

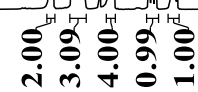

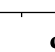

$\begin{array}{lll}9.5 & 9.0 & 8.5\end{array}$

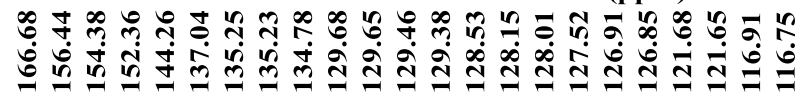

กุริำ กิ

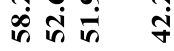

$\frac{7}{5}$

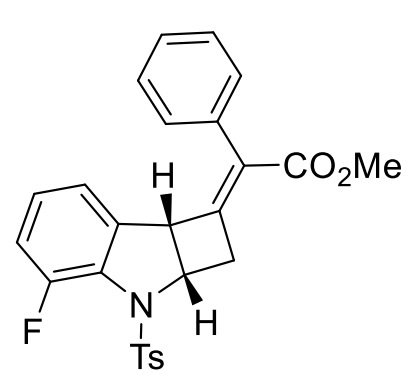

(E)-3pa

(125 MHz; in $\mathrm{CDCl}_{3}$ )

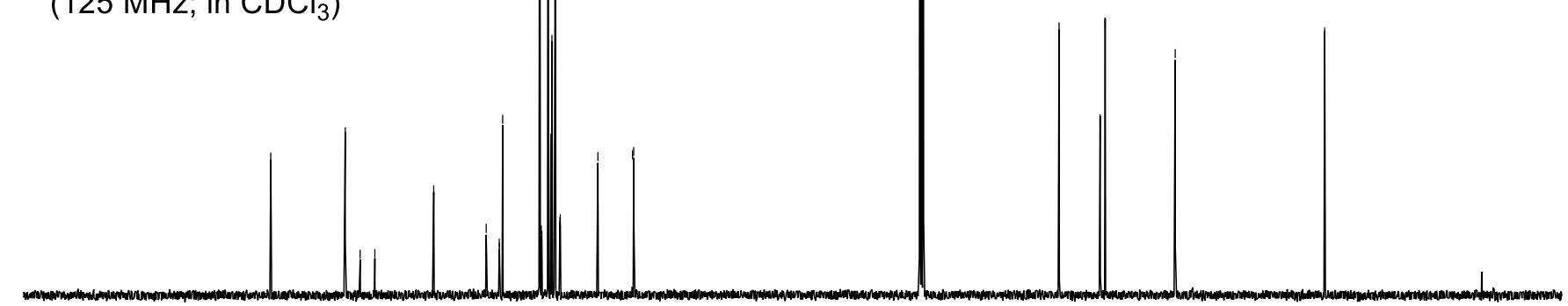




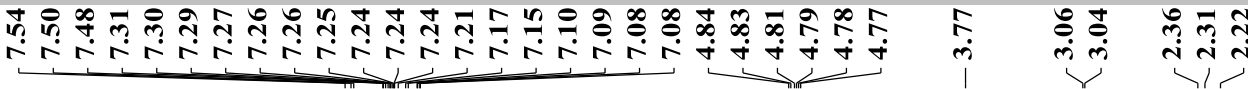

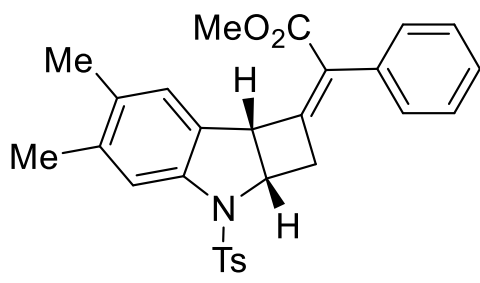

(Z)-3qa

$\left(500 \mathrm{MHz}\right.$; in $\mathrm{CDCl}_{3}$ )

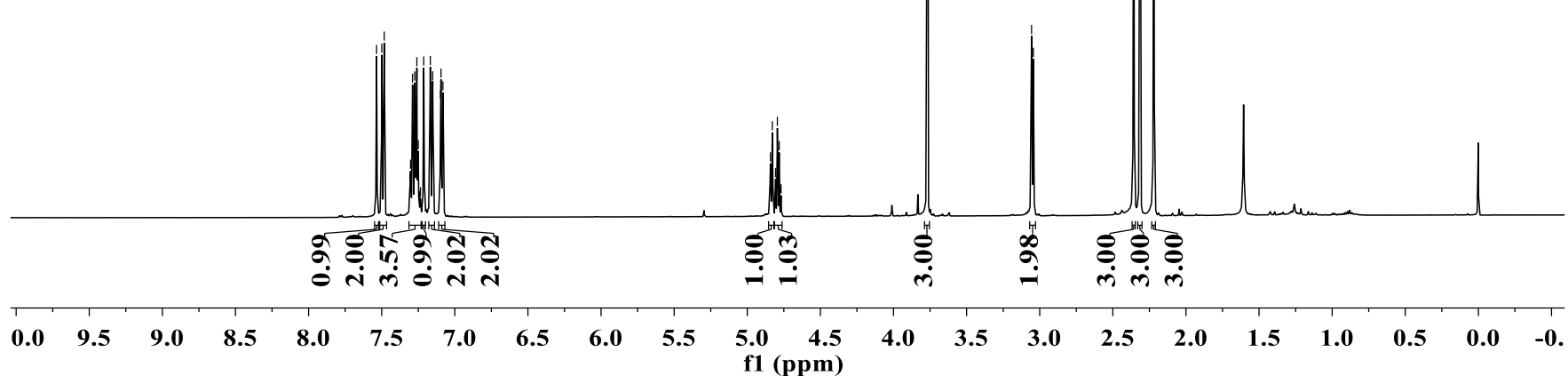

ㄱ.

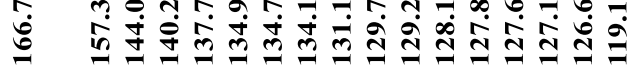

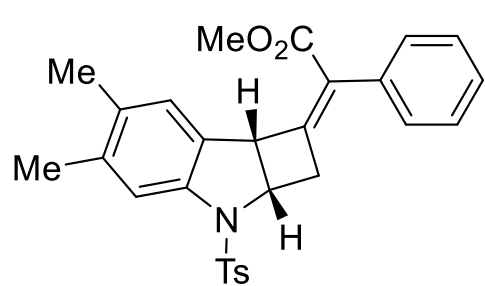

(Z)-3qa

$\left(125 \mathrm{MHz}\right.$; in $\mathrm{CDCl}_{3}$ ) 


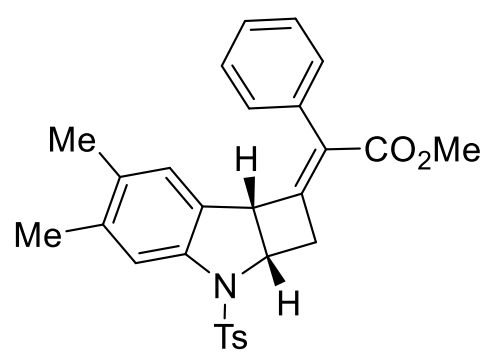

(E)-3qa

$\left(500 \mathrm{MHz}\right.$; in $\mathrm{CDCl}_{3}$ )

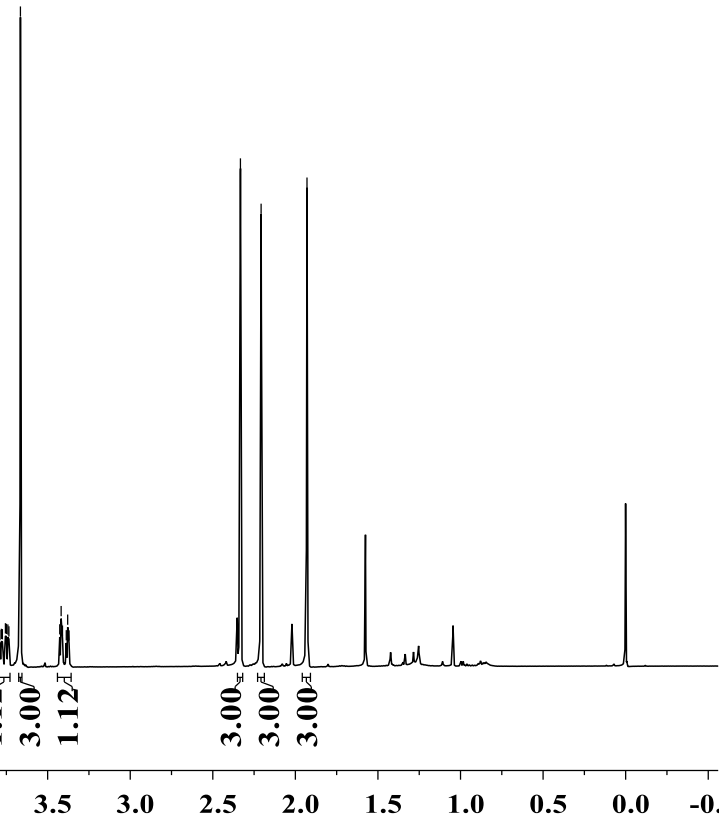

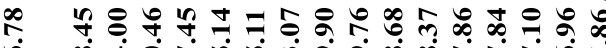

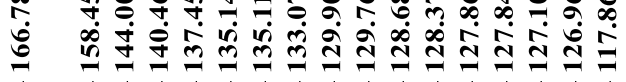

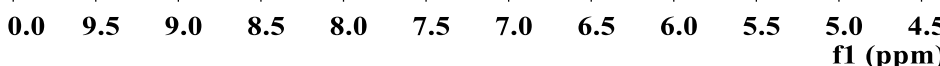
4.0

in

הับ<smiles>CCOC(=O)/C(=C1/C[C@@H]2[C@@H]1[C@@H]1[C@@H]2c2cc(C)c(C)cc2N1[13CH3])c1ccccc1</smiles>

(E)-3qa 
<smiles>COC/C(=C/c1ccccc1)[C@@H]1C[C@@H]2[C@@H]1c1cc(OC)c(Cl)cc1N2[13CH3]</smiles>

(Z)-3ra

$\left(500 \mathrm{MHz}\right.$; in $\mathrm{CDCl}_{3}$ )

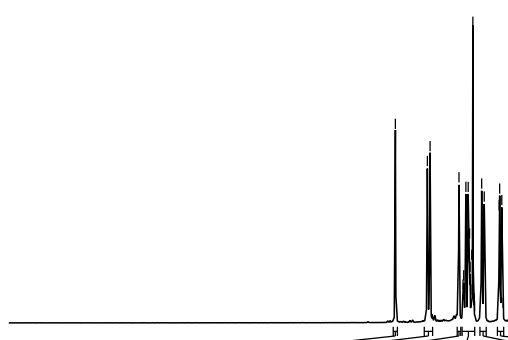

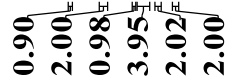

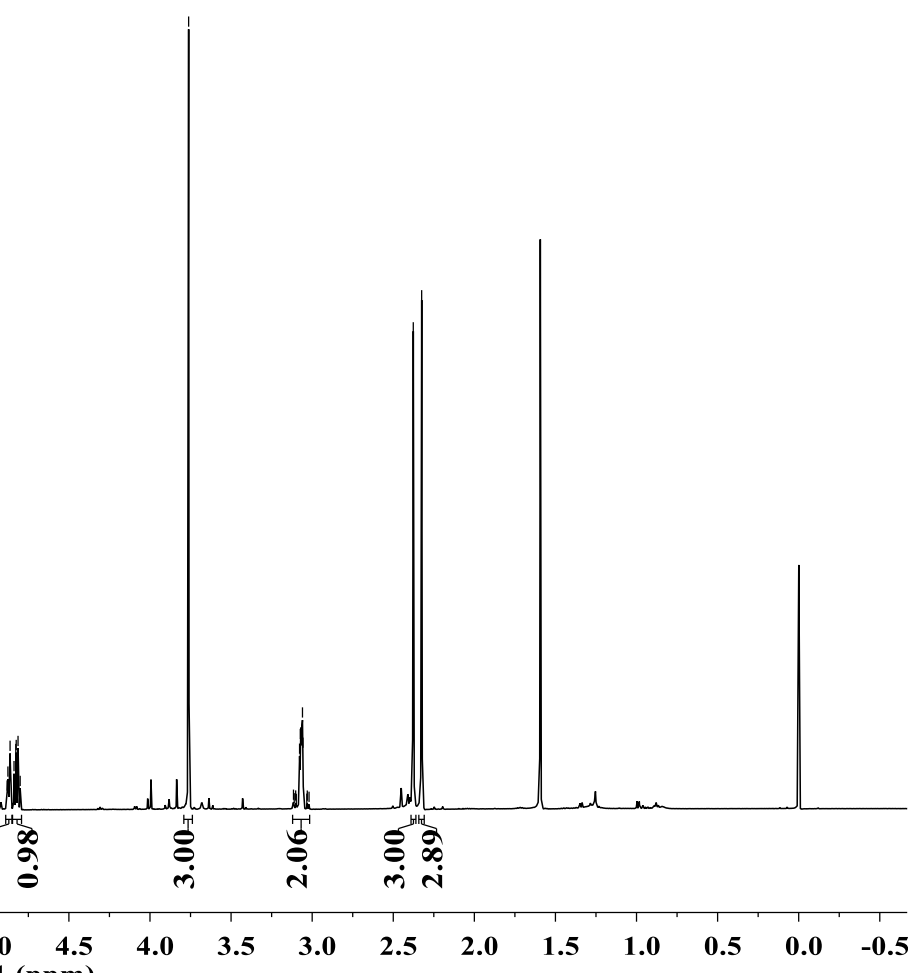

$\begin{array}{llllllllllll}10.0 & 9.5 & 9.0 & 8.5 & 8.0 & 7.5 & 7.0 & 6.5 & 6.0 & 5.5 & \begin{array}{l}5.0 \\ \text { f1 }\end{array}(\mathrm{ppm}) & 4.5\end{array}$

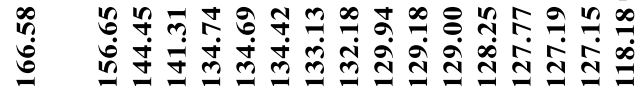

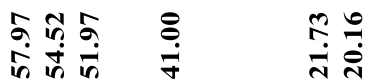<smiles>[Y8]N1c2cc(Cl)c(OC)cc2[C@H]2/C(=C(\C(=O)OC)c3ccccc3)C[C@@H]2[C@@H]1C</smiles>

(Z)-3ra

$\left(125 \mathrm{MHz}\right.$; in $\left.\mathrm{CDCl}_{3}\right)$

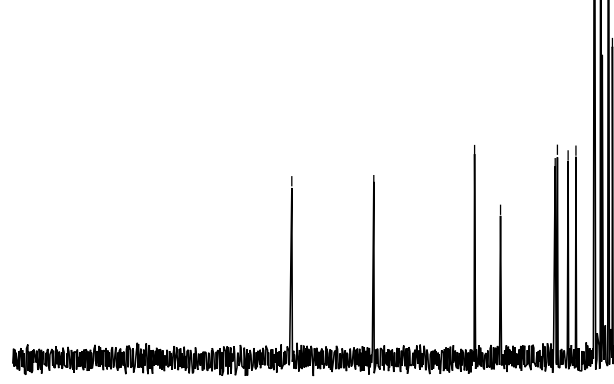




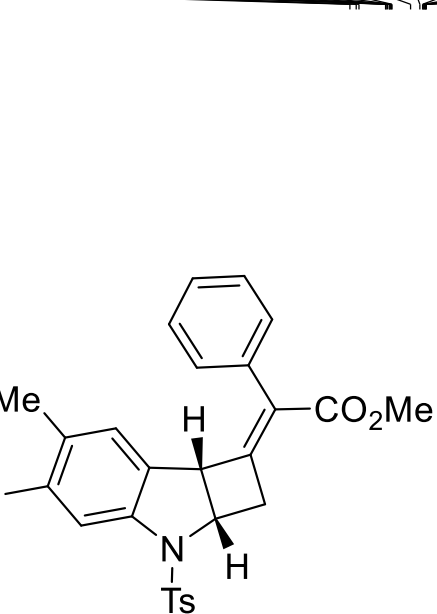

(E)-3ra

$\left(500 \mathrm{MHz}\right.$; in $\left.\mathrm{CDCl}_{3}\right)$

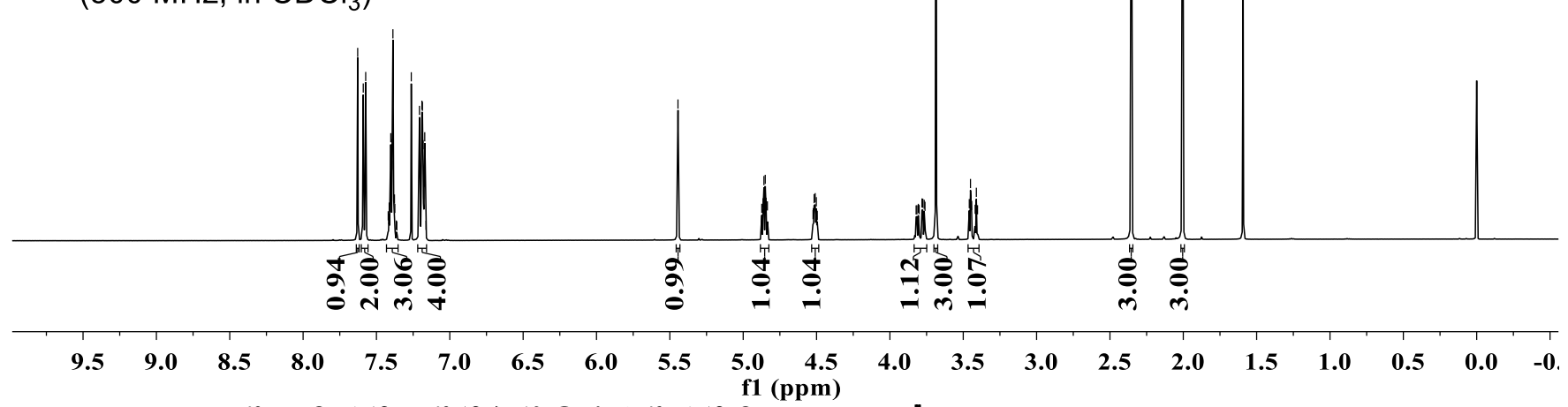

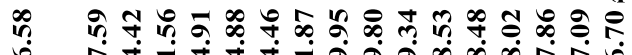

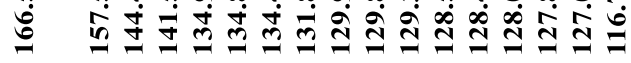

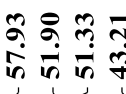

ำ

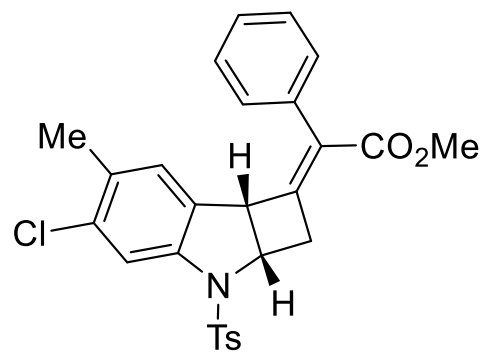

(E)-3ra

(125 $\mathrm{MHz}$; in $\mathrm{CDCl}_{3}$ ) 
<smiles>COC(=C1C[C@H]2[C@H]1c1cc(F)cc(C)c1N2[13CH3])c1ccccc1</smiles>

(Z)-3sa

$\left(500 \mathrm{MHz} ;\right.$ in $\left.\mathrm{CDCl}_{3}\right)$

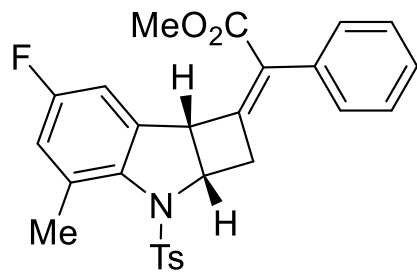

(Z)-3sa

(125 $\mathrm{MHz}$; in $\mathrm{CDCl}_{3}$ )

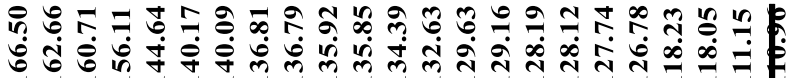

₹

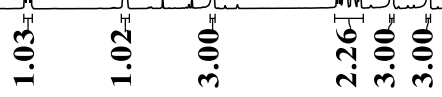

9.5

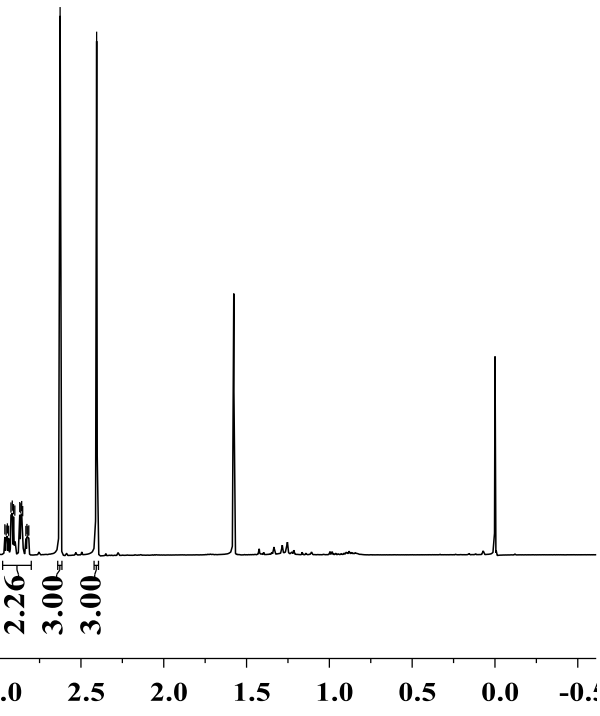

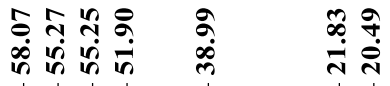

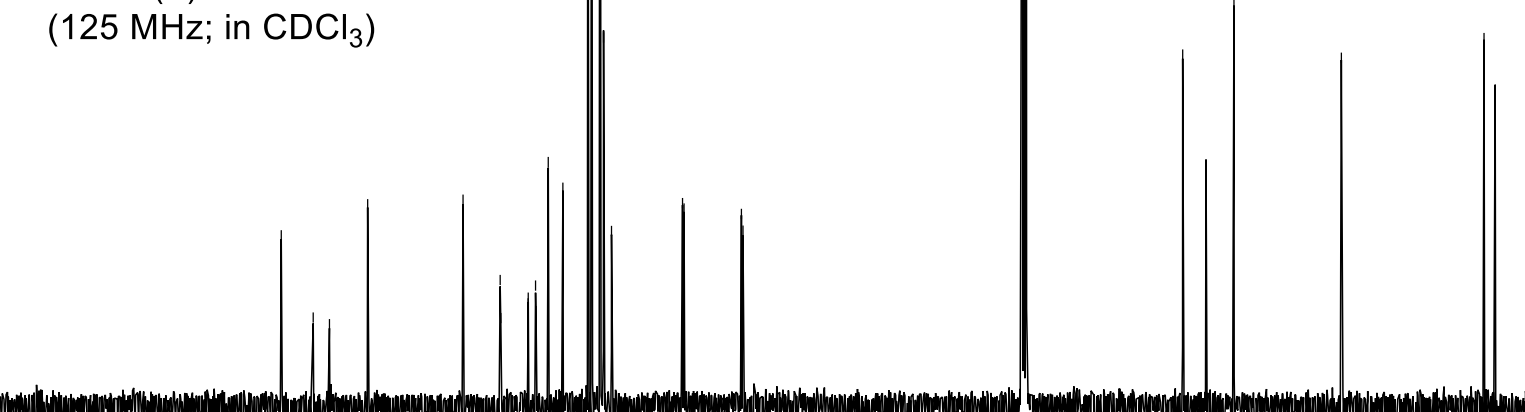


<smiles>CC(=O)/C(=C1/C[C@@H]2[C@H]1c1cc(F)cc(C)c1N2[13CH3])c1ccccc1</smiles>

$(E)$-3sa

$\left(500 \mathrm{MHz}\right.$; in $\mathrm{CDCl}_{3}$ )

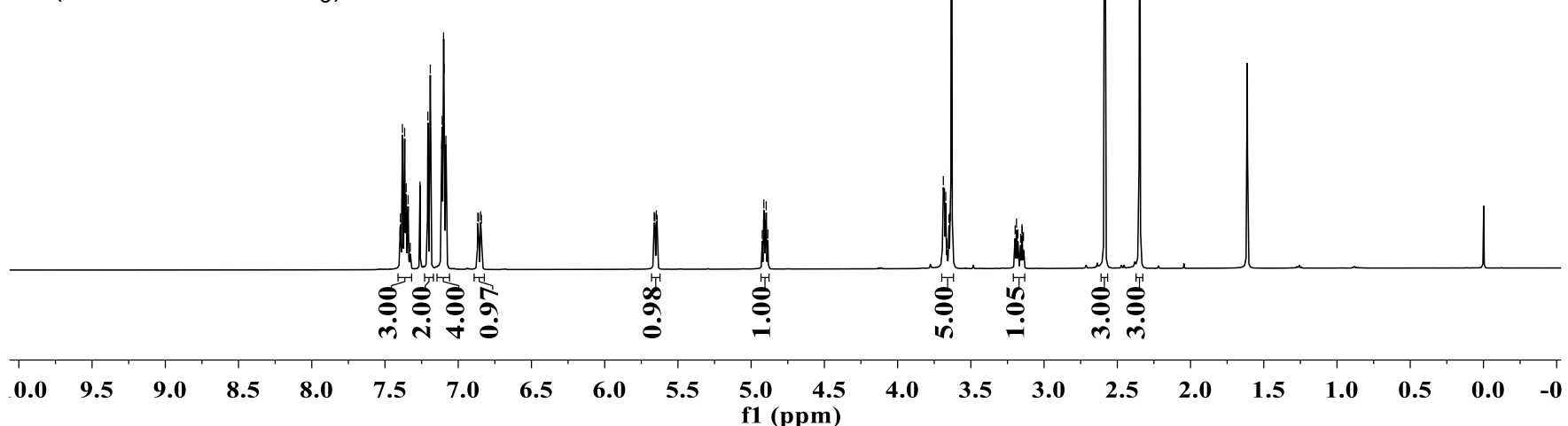

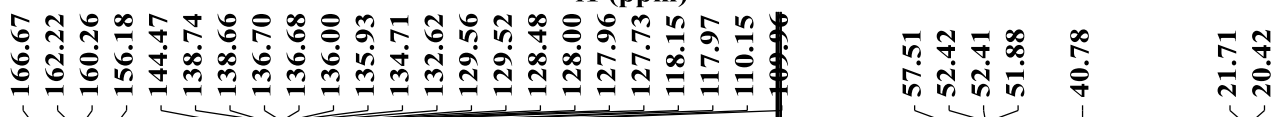

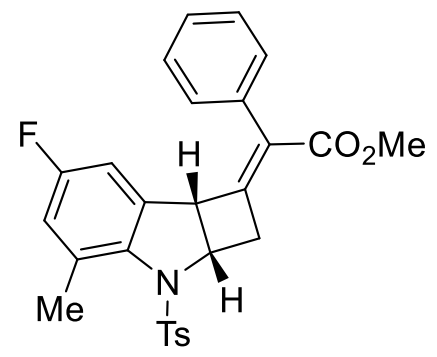

(E)-3sa

(125 MHz; in $\mathrm{CDCl}_{3}$ ) 


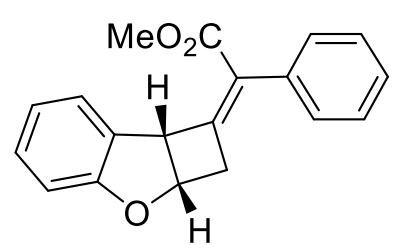

(Z)-3ta

(500 MHz; in $\mathrm{CDCl}_{3}$ )

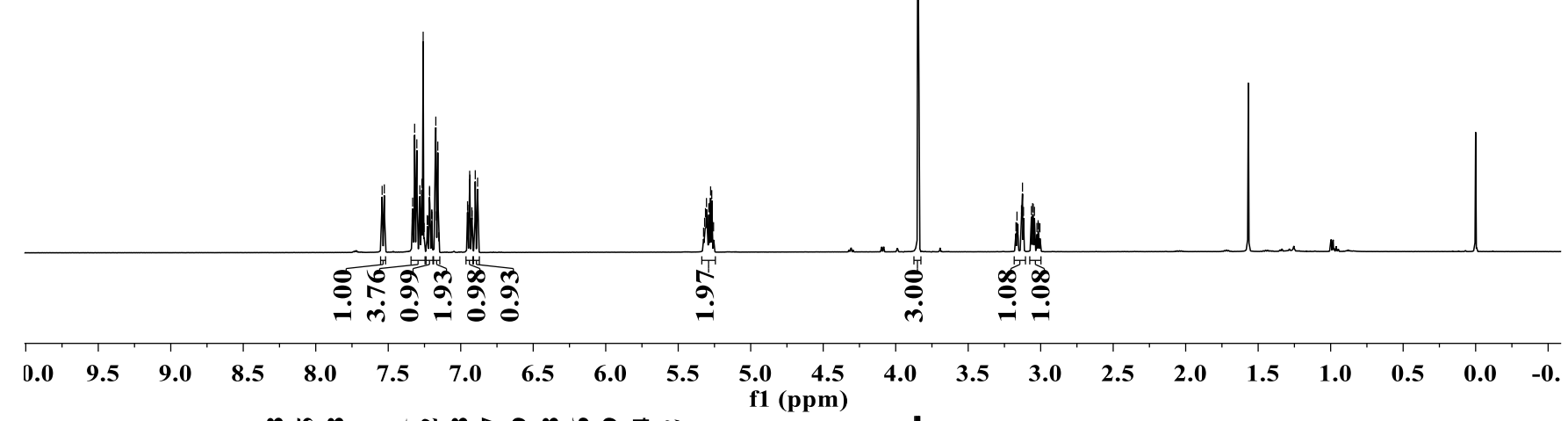

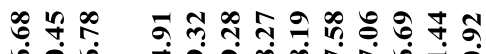

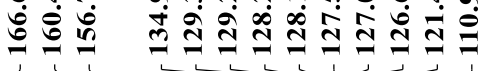

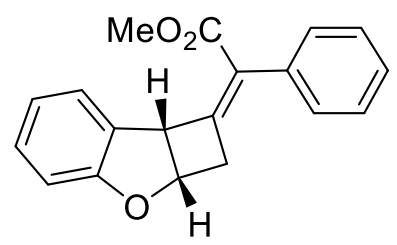

(Z)-3ta

$\left(125 \mathrm{MHz}\right.$; in $\mathrm{CDCl}_{3}$ ) 


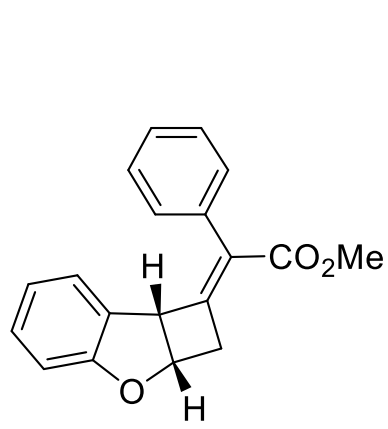

(E)-3ta

(500 MHz; in $\mathrm{CDCl}_{3}$ )

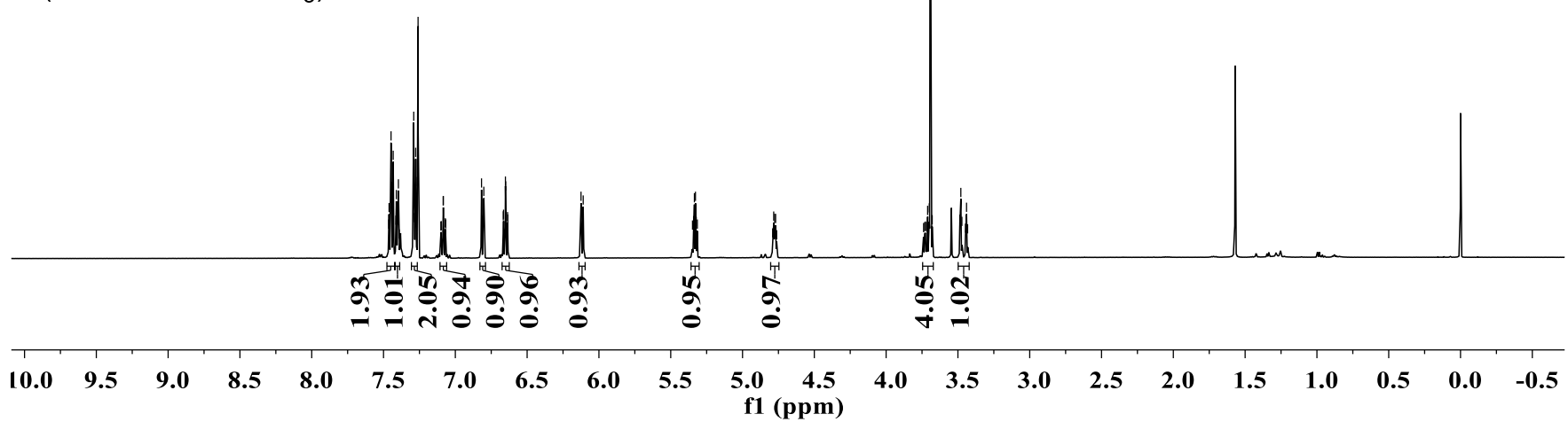

ธकำ

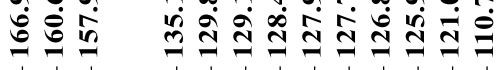

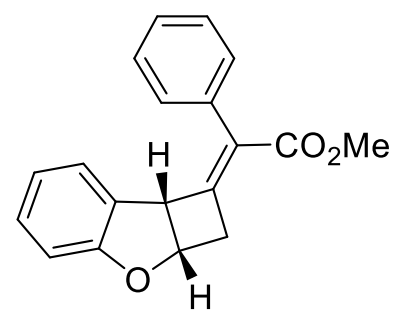

(E)-3ta

$\left(125 \mathrm{MHz}\right.$; in $\mathrm{CDCl}_{3}$ )

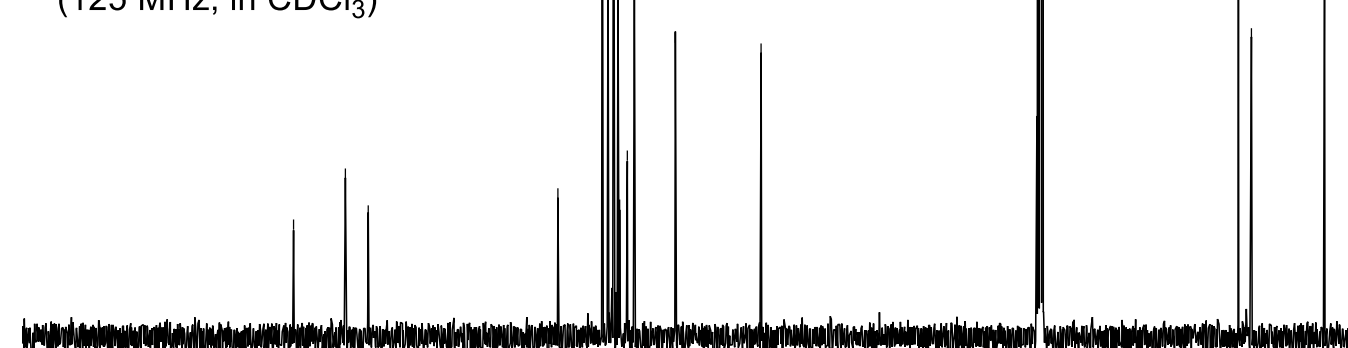




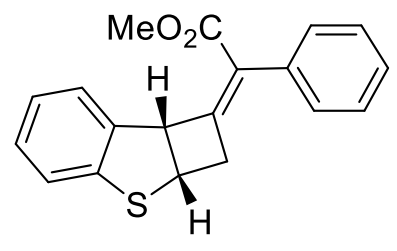

(Z)-3ua

$\left(500 \mathrm{MHz}\right.$; in $\mathrm{CDCl}_{3}$ )

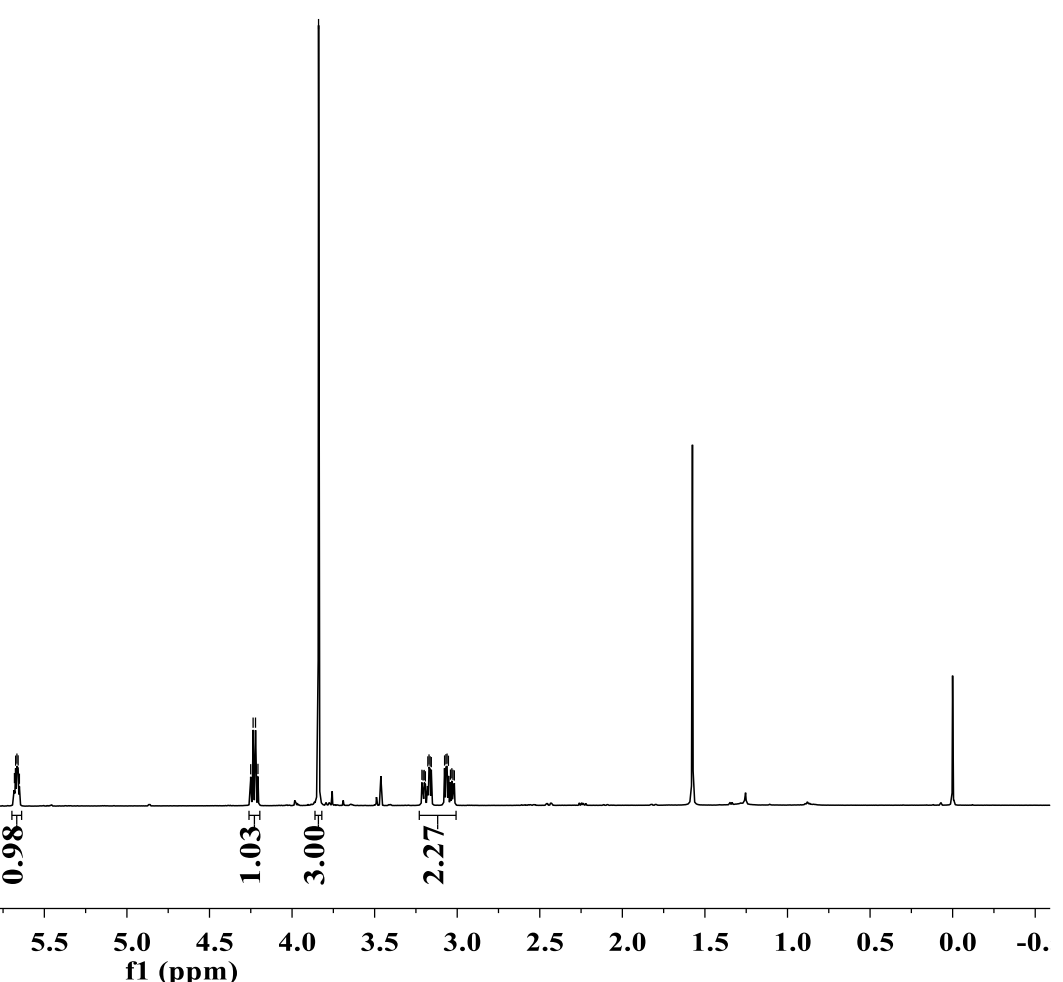

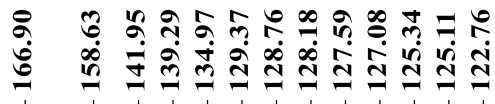

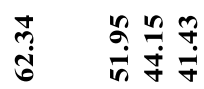

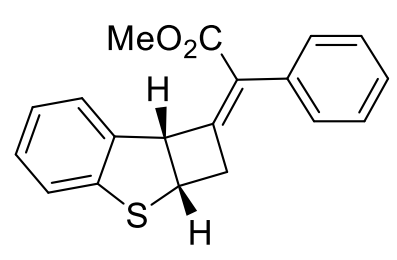

(Z)-3ua

$\left(125 \mathrm{MHz}\right.$; in $\left.\mathrm{CDCl}_{3}\right)$

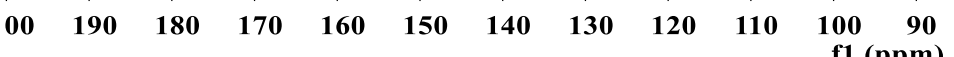

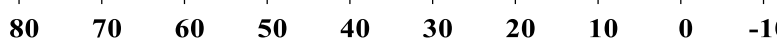




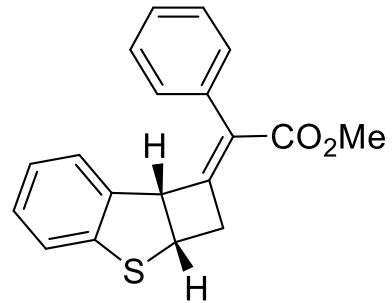

(E)-3ua

$\left(500 \mathrm{MHz}\right.$; in $\left.\mathrm{CDCl}_{3}\right)$

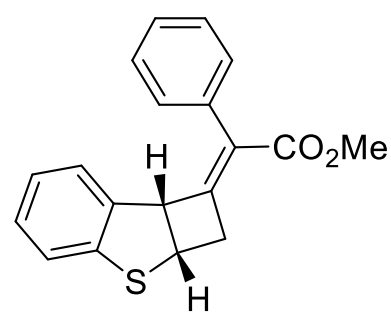

(E)-3ua

$\left(125 \mathrm{MHz}\right.$; in $\left.\mathrm{CDCl}_{3}\right)$

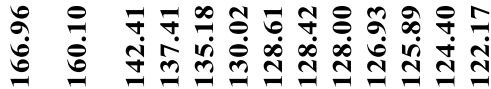

\section{ڤேं}

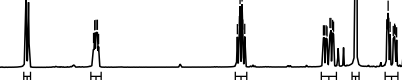
这

$\begin{array}{llllllllllll}9.5 & 9.0 & 8.5 & 8.0 & 7.5 & 7.0 & 6.5 & 6.0 & 5.5 & 5.0 & 4.5 \\ \end{array}$ $\begin{array}{lll}4.0 & 3.5 & 3.0\end{array}$ 
<smiles>C=CCc1ccccc1C#CC(=Cc1ccccc1)C(C)=O</smiles>

$4 a$

$\left(500 \mathrm{MHz}\right.$; in $\left.\mathrm{CDCl}_{3}\right)$<smiles>C=CCc1ccccc1C#CC(=Cc1ccccc1)C(C)=O</smiles>

$4 a$

(125 MHz; in $\mathrm{CDCl}_{3}$ )

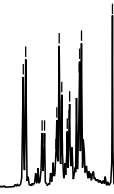

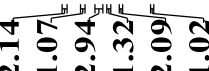

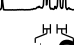

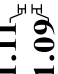

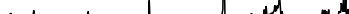

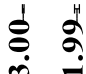

$\begin{array}{lllllllllllllllllllllllllllll}0.0 & 9.5 & 9.0 & 8.5 & 8.0 & 7.5 & 7.0 & 6.5 & 6.0 & 5.5 & 5.0 & 4.5 & 4.0 & 3.5 & 3.0 & 2.5 & 2.0 & 1.5 & 1.0 & 0.5 & 0.0 & -0\end{array}$

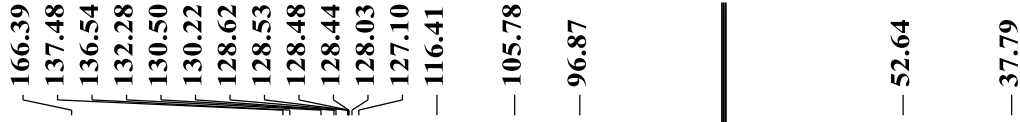

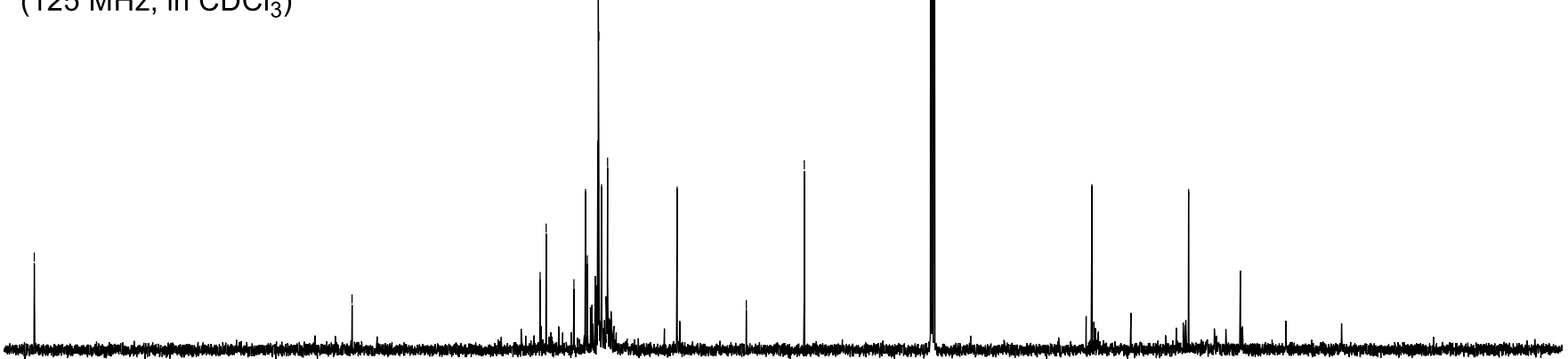

21020

$\begin{array}{llll}190 & 180 & 170 & 16\end{array}$

150140

120

1010090

$80 \quad 70 \quad 60$

504

030

20

O -10 


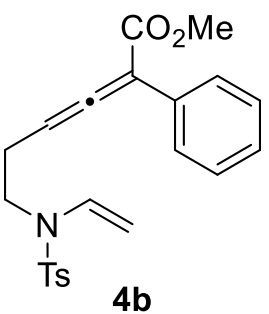

$\left(500 \mathrm{MHz}\right.$; in $\left.\mathrm{CDCl}_{3}\right)$

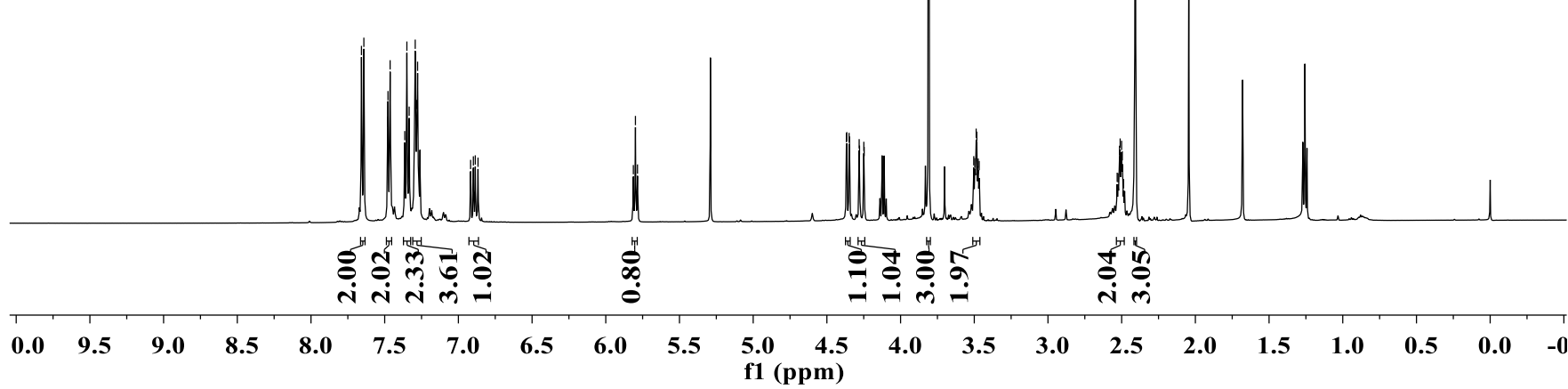

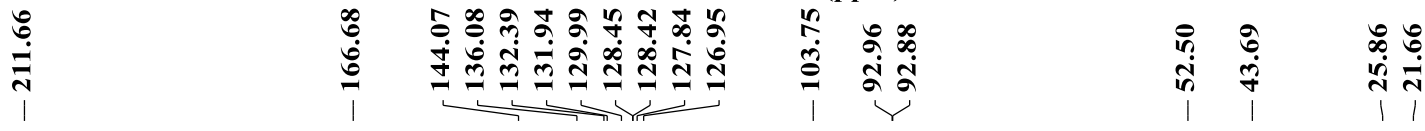

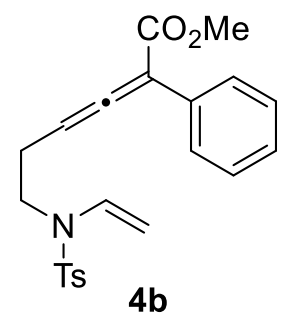

(125 MHz; in $\mathrm{CDCl}_{3}$ )

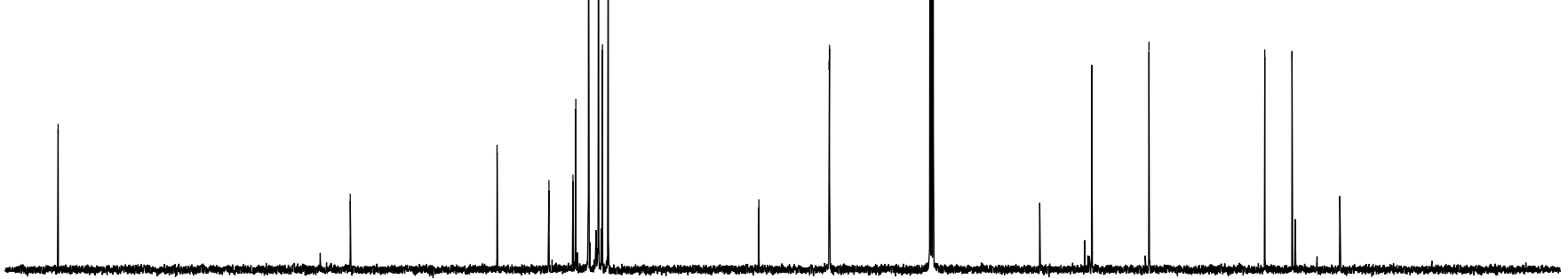

$\begin{array}{lllllllllllllllllllllllllllllllll}210 & 200 & 190 & 180 & 170 & 160 & 150 & 140 & 130 & 120 & 110 & \begin{array}{c}100 \\ \text { f1 }\end{array}(\mathrm{ppm}) & 90 & 80 & 70 & 60 & 50 & 40 & 30 & 20 & 10 & 0 & -10 & \end{array}$ 


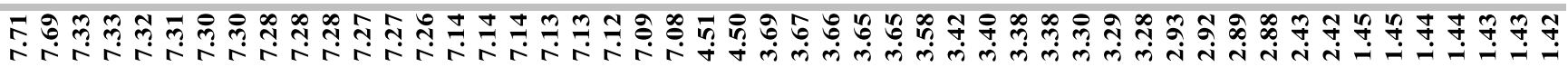

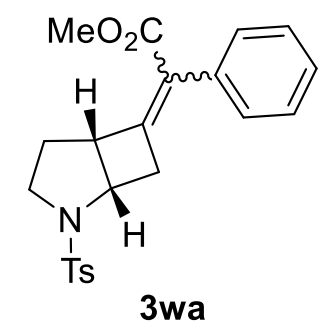

(500 MHz; in $\mathrm{CDCl}_{3}$ )
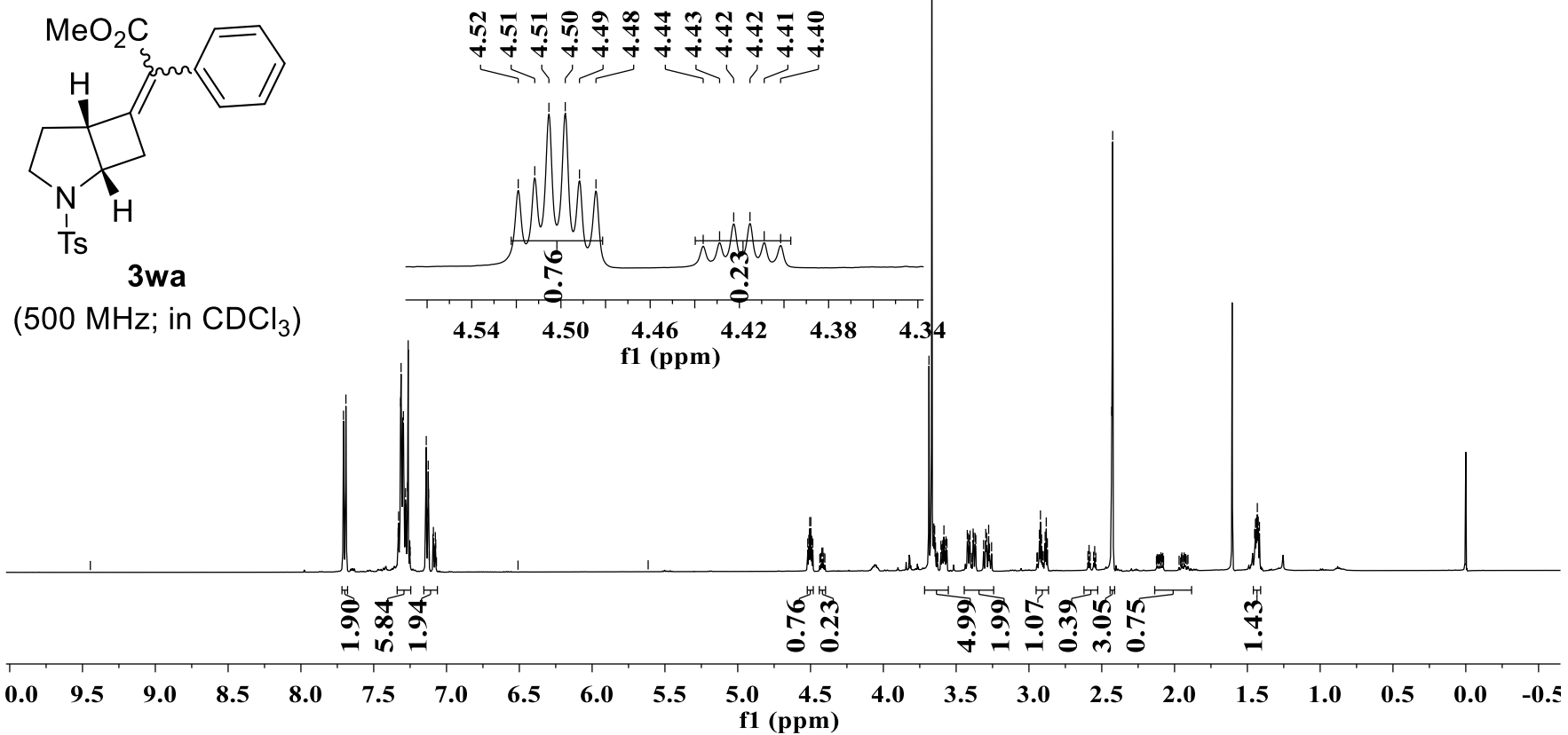

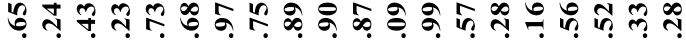

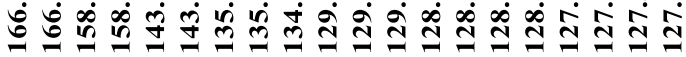

ว

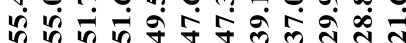

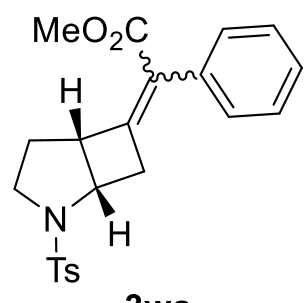

3wa

(125 MHz; in $\mathrm{CDCl}_{3}$ )

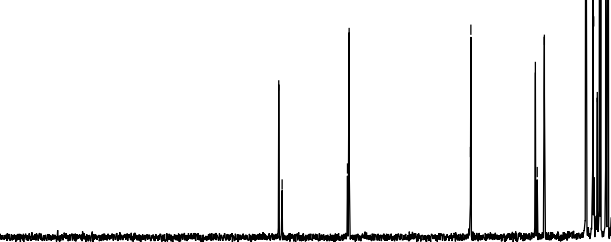




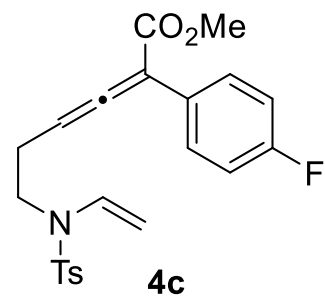

$\left(500 \mathrm{MHz} ;\right.$ in $\left.\mathrm{CDCl}_{3}\right)$
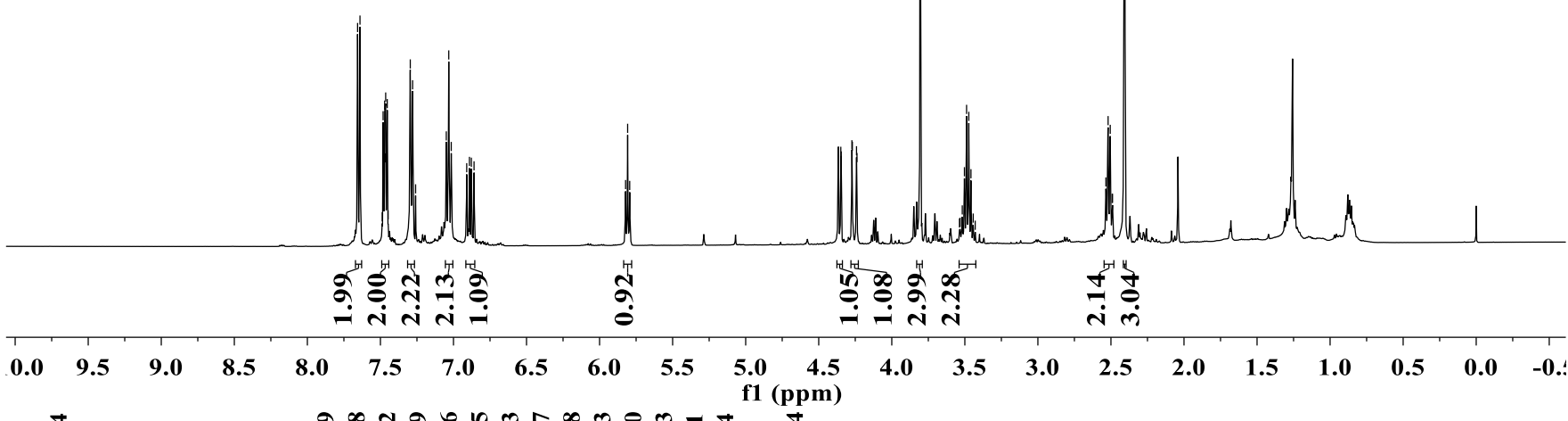

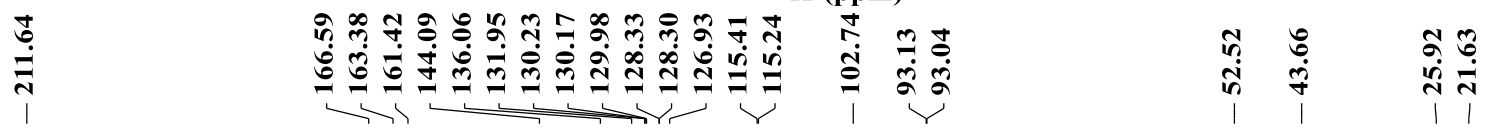

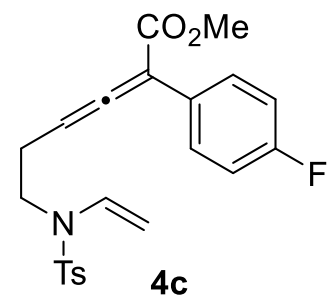

$\left(125 \mathrm{MHz}\right.$; in $\left.\mathrm{CDCl}_{3}\right)$

$\begin{array}{llllllllllll}210 & 200 & 190 & 180 & 170 & 160 & 150 & 140 & 130 & 120 & 110 & 100\end{array}$ 


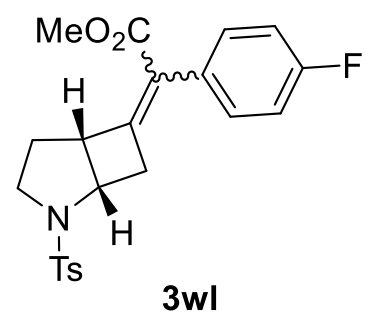

$\left(500 \mathrm{MHz}\right.$; in $\left.\mathrm{CDCl}_{3}\right)$

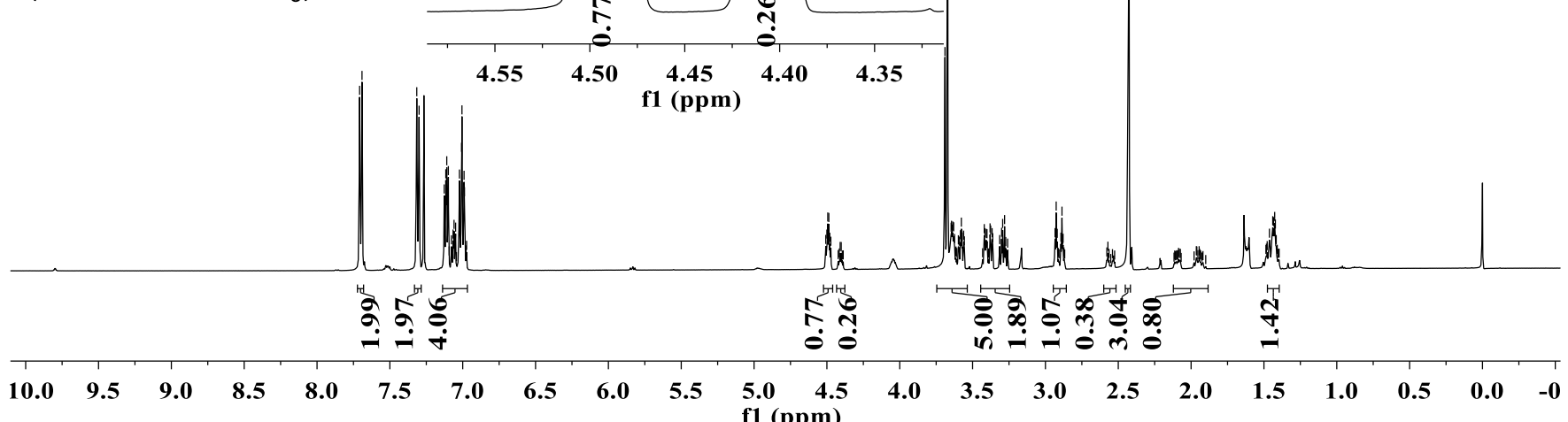

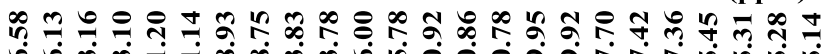

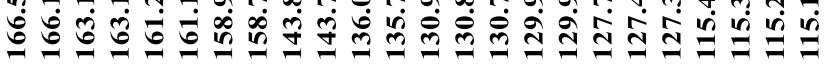

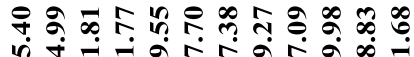

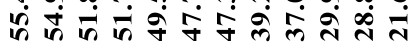

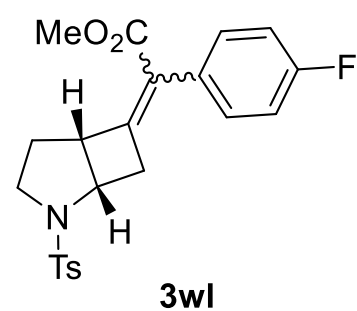

$\left(125 \mathrm{MHz}\right.$; in $\left.\mathrm{CDCl}_{3}\right)$

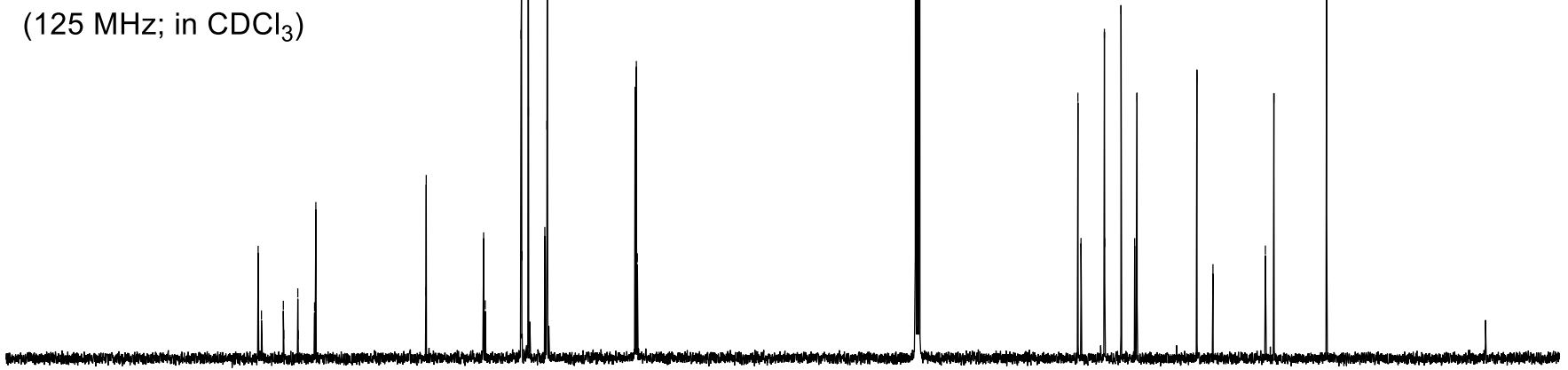




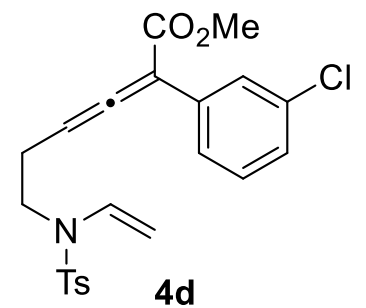

(500 MHz; in $\mathrm{CDCl}_{3}$ )
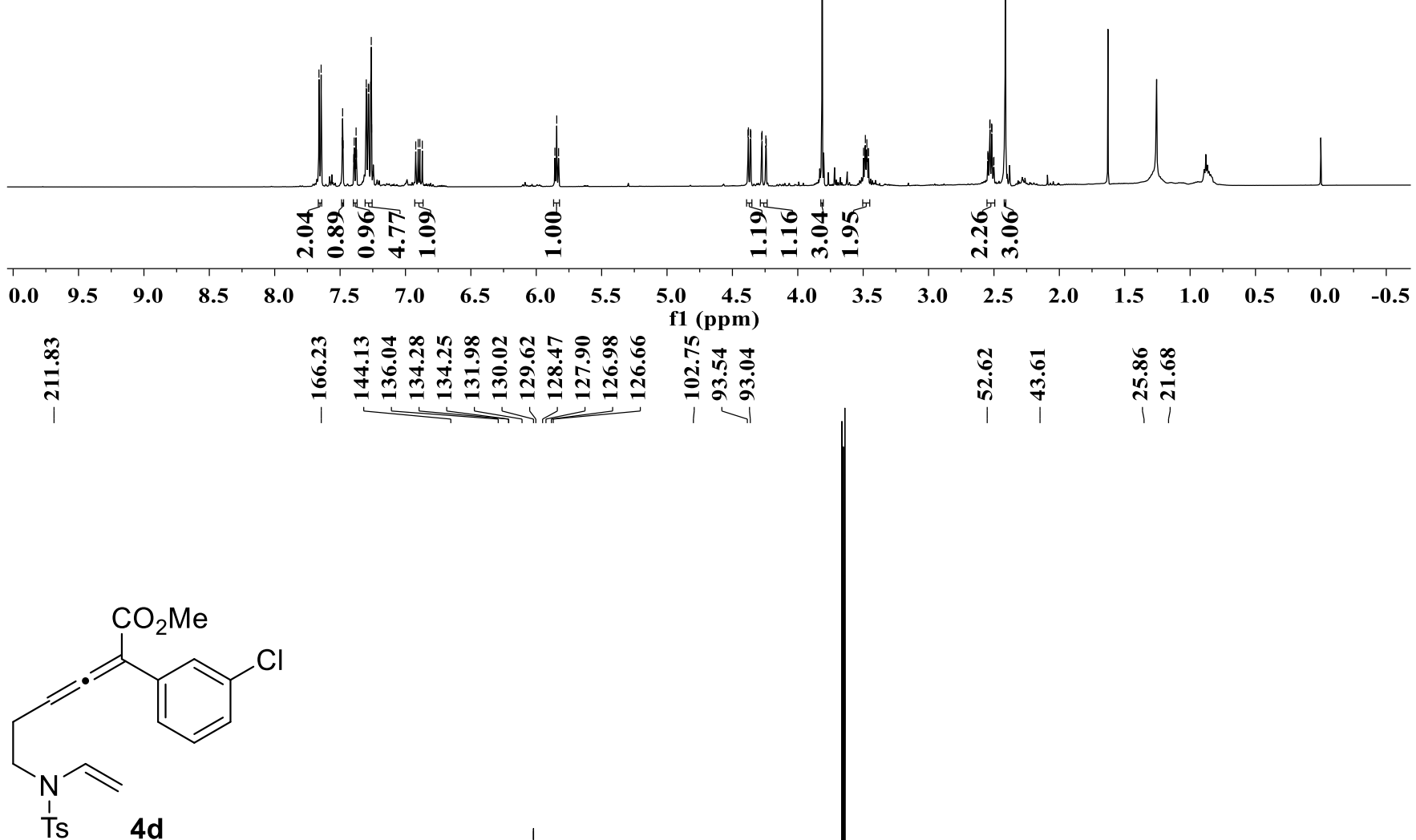

(125 MHz; in $\mathrm{CDCl}_{3}$ )

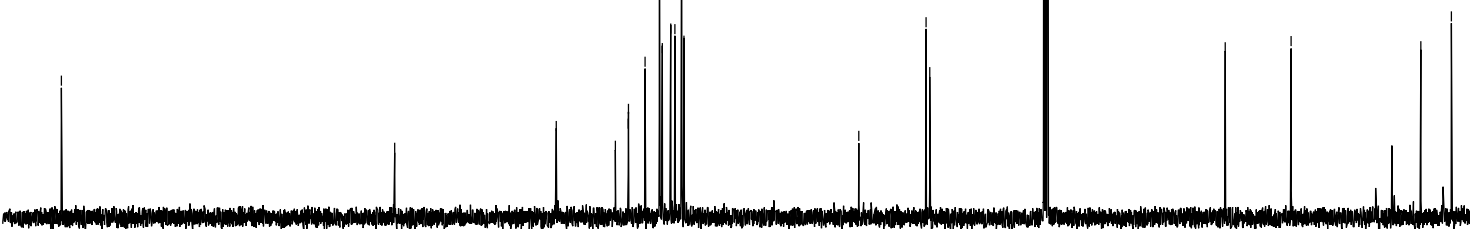

$\begin{array}{lllllllllllllllllllllllllll}210 & 200 & 190 & 180 & 170 & 160 & 150 & 140 & 130 & 120 & 110 & 100 & 90 & 80 & 70 & 60 & 50 & 40 & 30 & 20 & 10 & 0 & -10 & \end{array}$ f1 (ppm) 
<smiles>COC(=O)[C@H]1C[C@H]2[C@@H]1CCN2[13CH3]</smiles>

$3 w q$

$\left(500 \mathrm{MHz}\right.$; in $\left.\mathrm{CDCl}_{3}\right)$

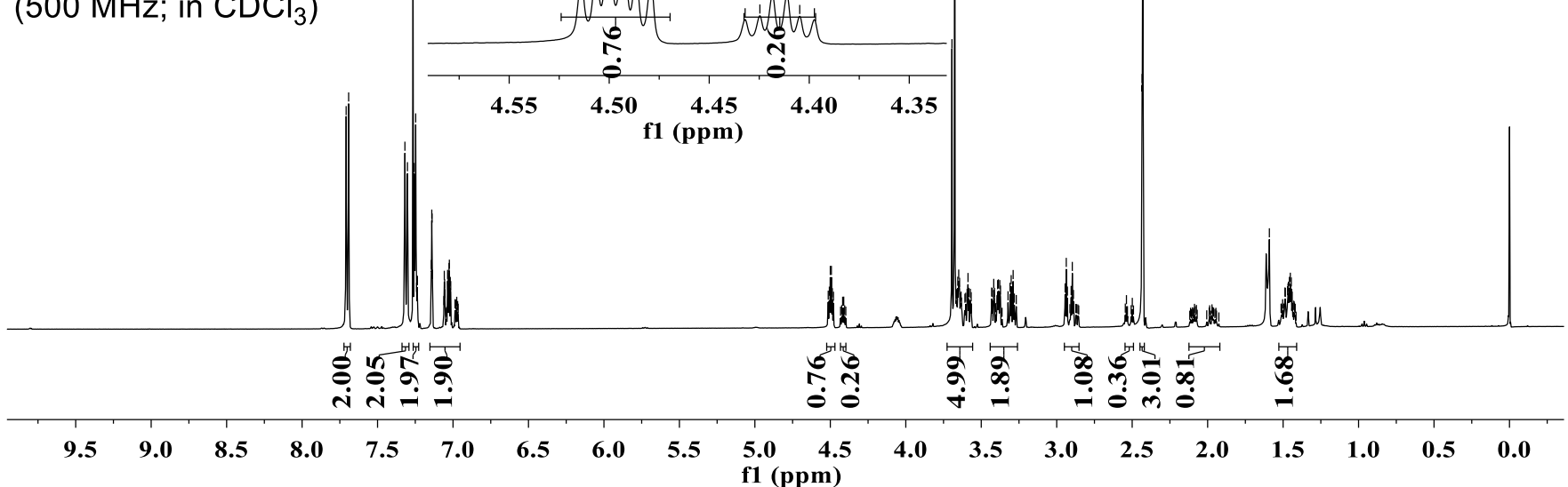

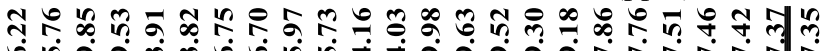

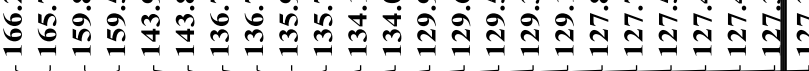<smiles>COC(=O)c1cccc(Cl)c1</smiles>

3wq

$\left(125 \mathrm{MHz}\right.$; in $\left.\mathrm{CDCl}_{3}\right)$

\section{倌}

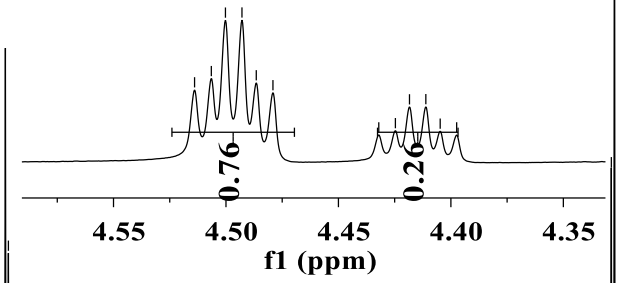

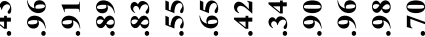

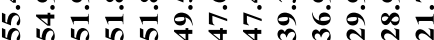




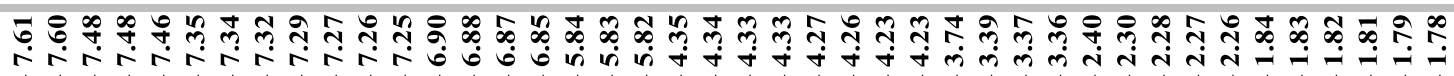

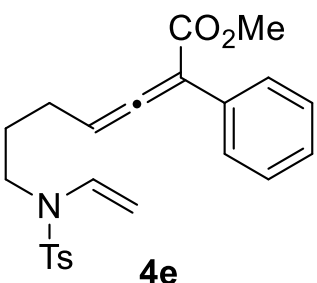

(500 $\mathrm{MHz}$; in $\mathrm{CDCl}_{3}$ )
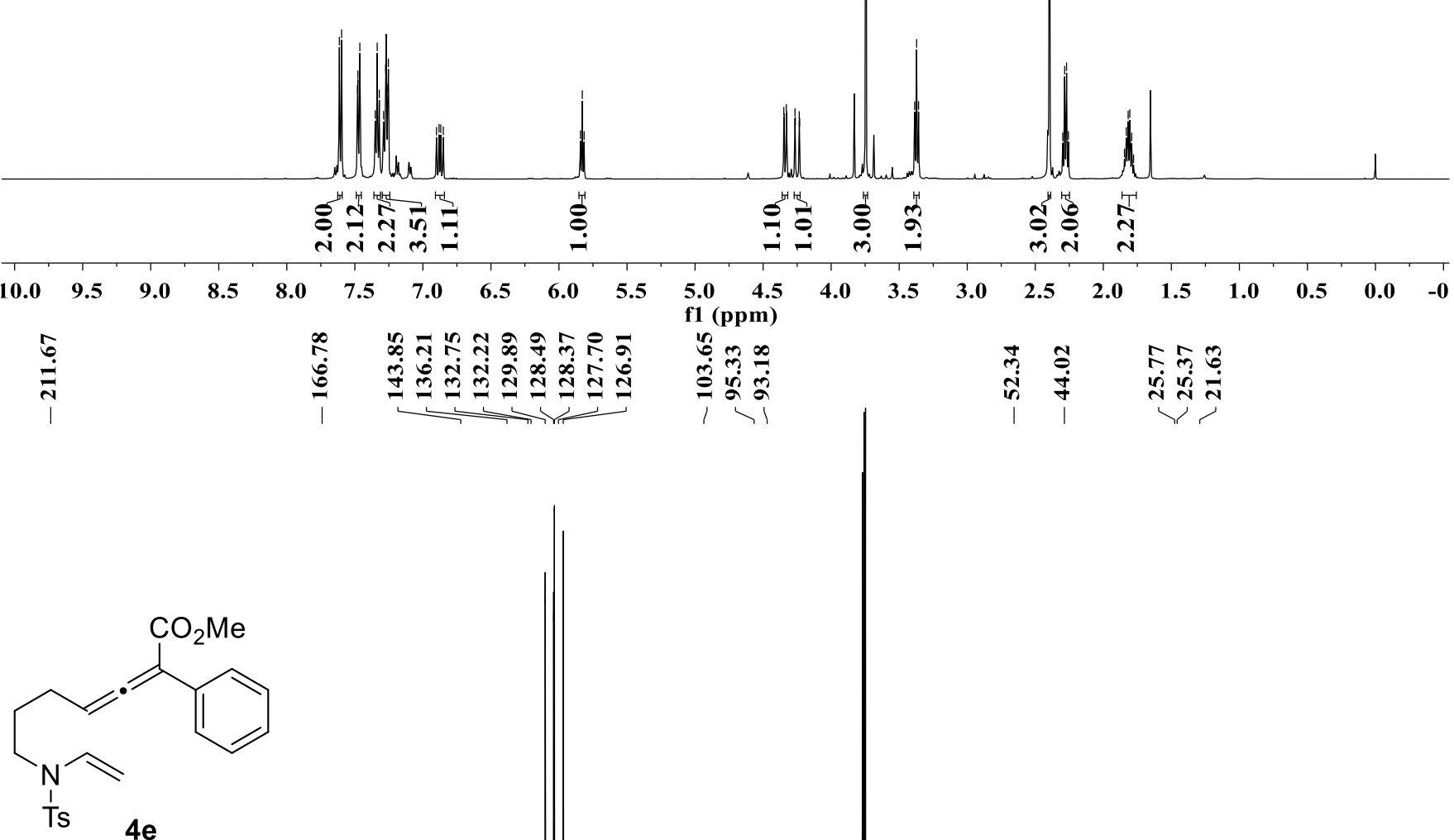

(125 $\mathrm{MHz}$; in $\mathrm{CDCl}_{3}$ )

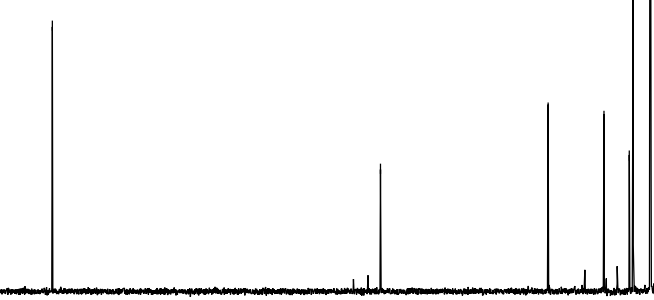

$\begin{array}{lllllllllllllllllllllll}210 & 200 & 190 & 180 & 170 & 160 & 150 & 140 & 130 & 120 & 110 & 100 & 90 & 80 & 70 & 60 & 50 & 40 & 30 & 20 & 10 & 0 & -10\end{array}$ f1 (ppm) 


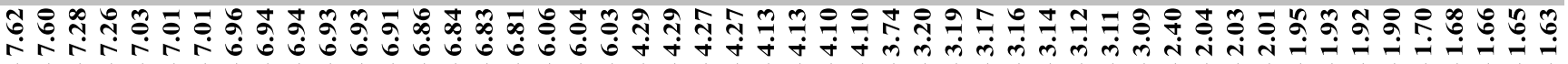

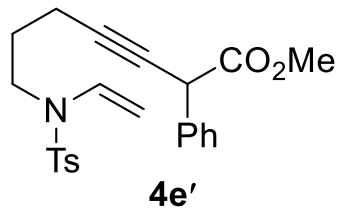

$\left(500 \mathrm{MHz}\right.$; in $\left.\mathrm{CDCl}_{3}\right)$

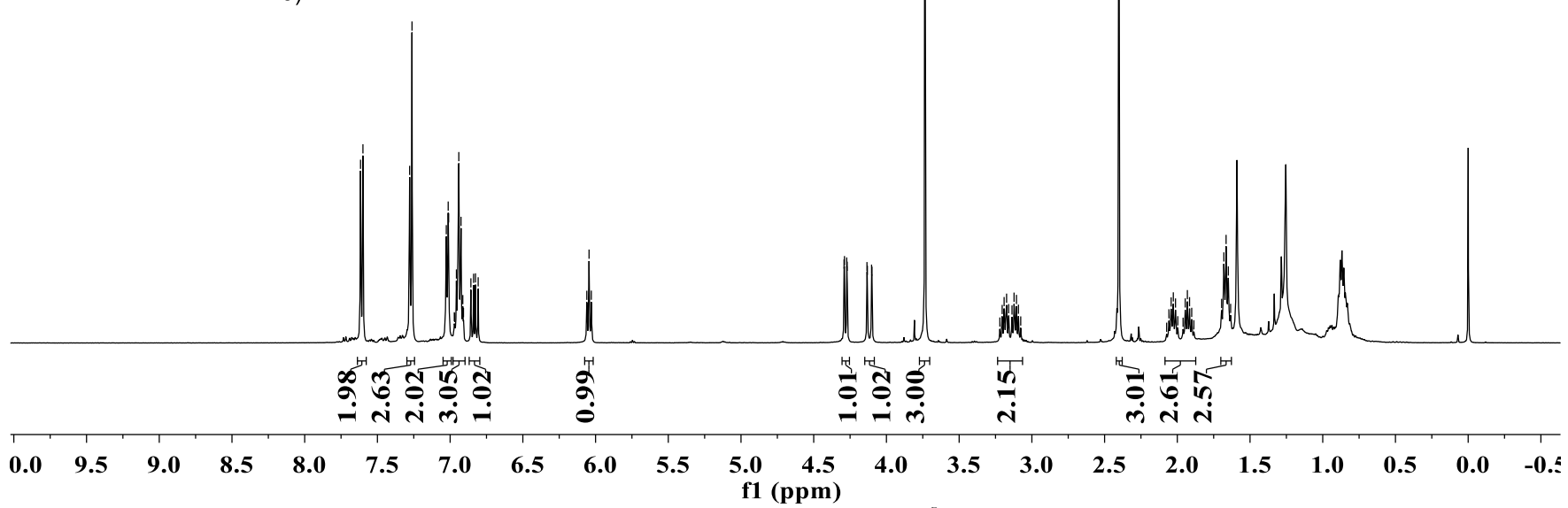

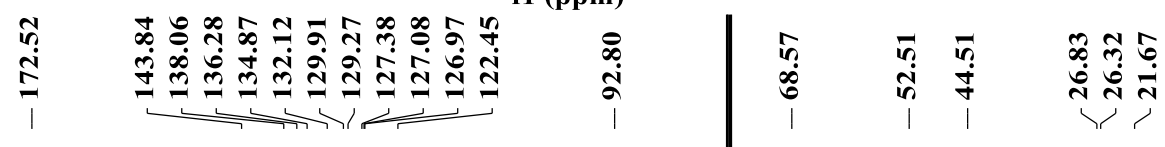

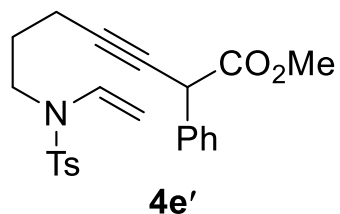

$\left(125 \mathrm{MHz} ;\right.$ in $\mathrm{CDCl}_{3}$ )

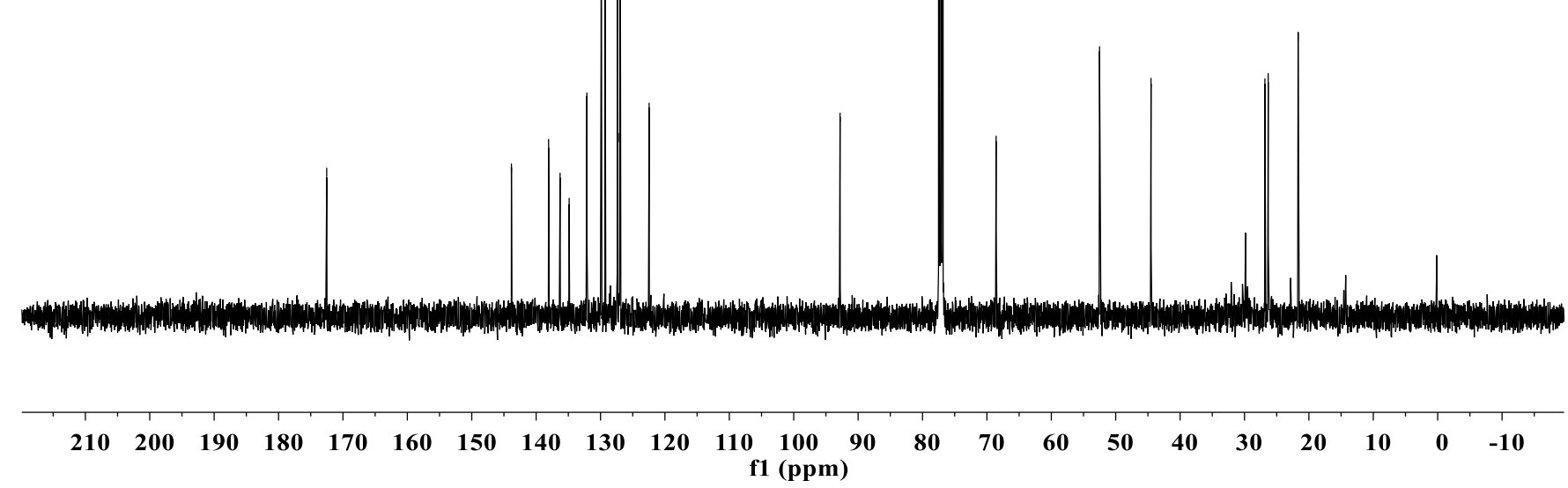




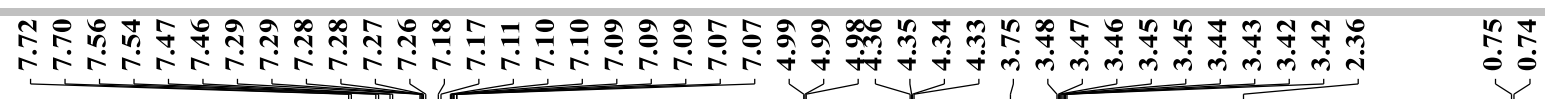

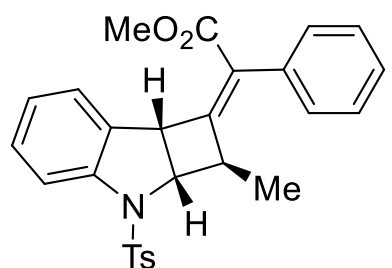

(Z)-3ya

$\left(500 \mathrm{MHz}\right.$; in $\mathrm{CDCl}_{3}$ )

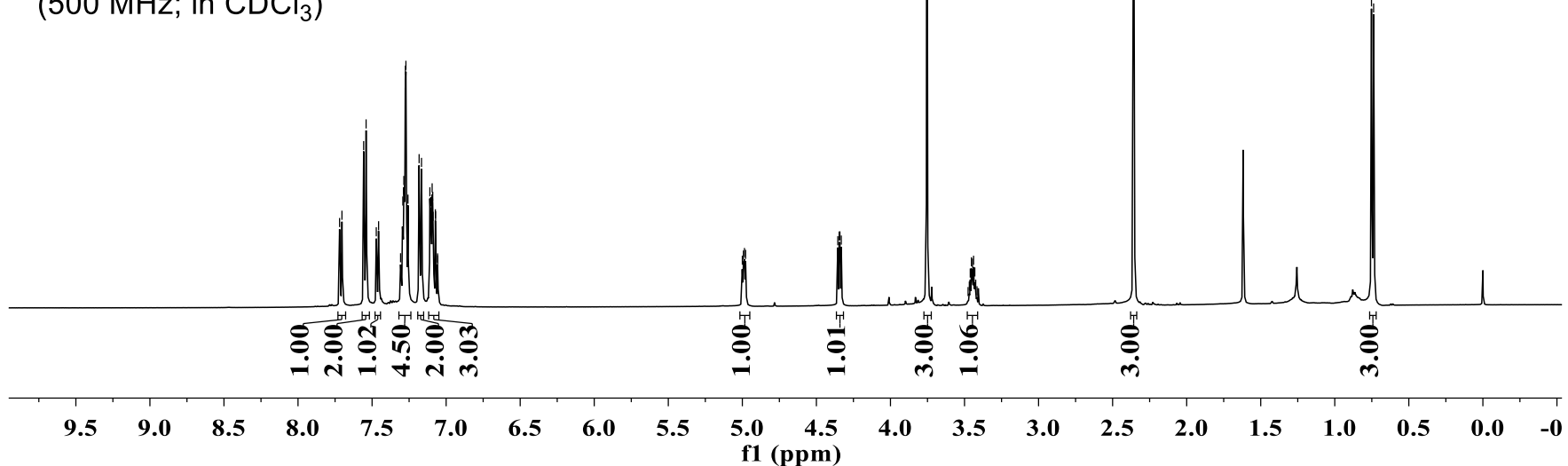

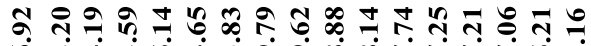

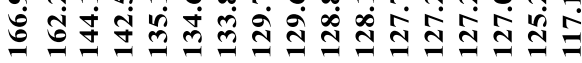

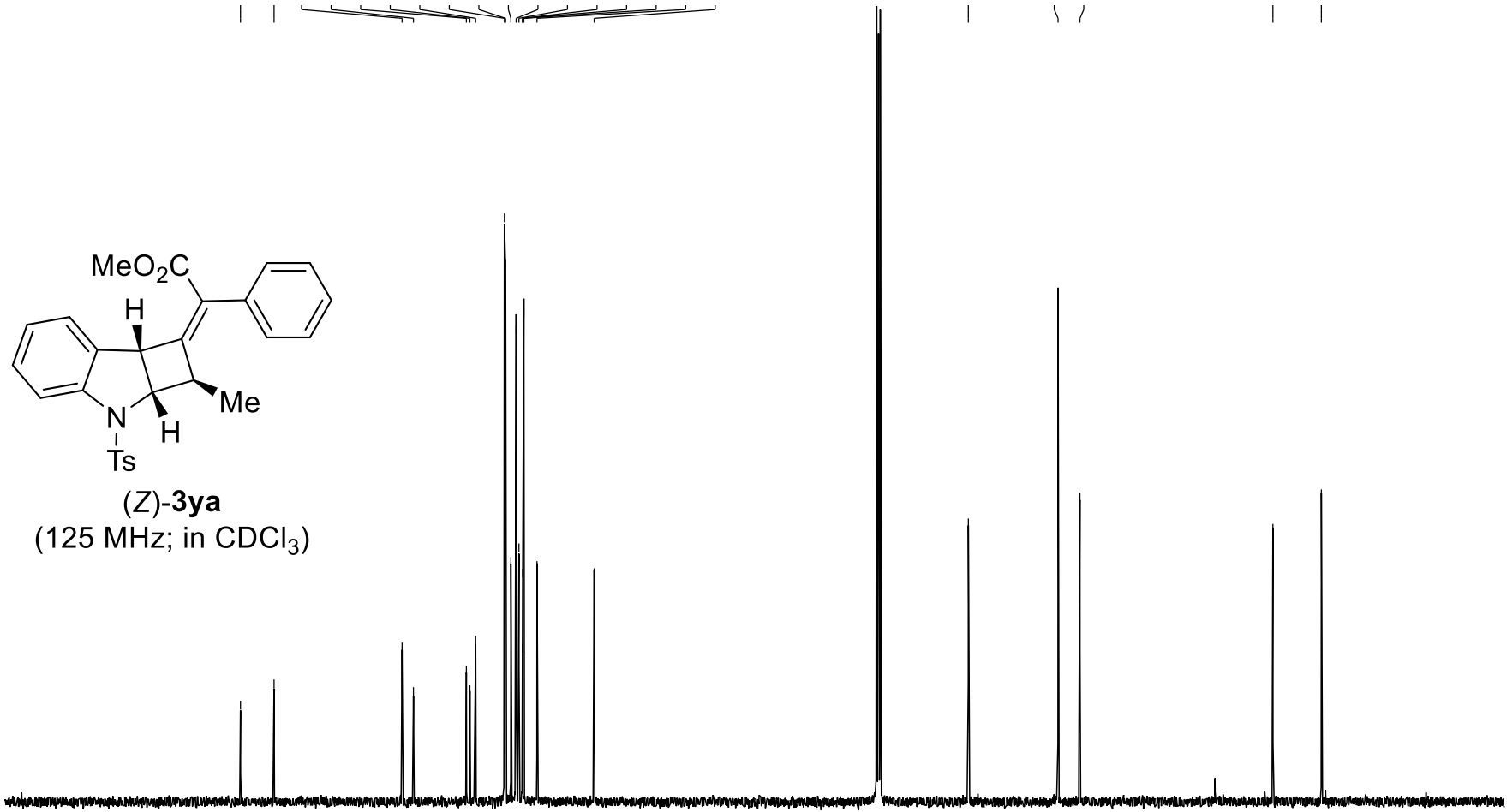




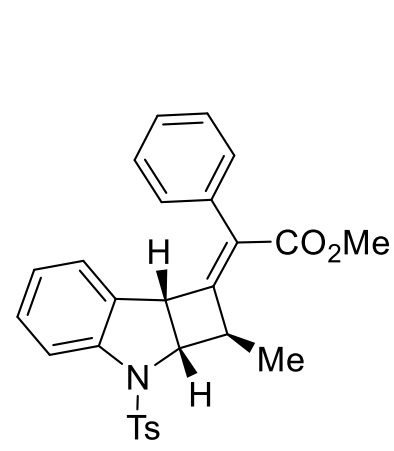

(E)-3ya

$\left(500 \mathrm{MHz}\right.$; in $\left.\mathrm{CDCl}_{3}\right)$

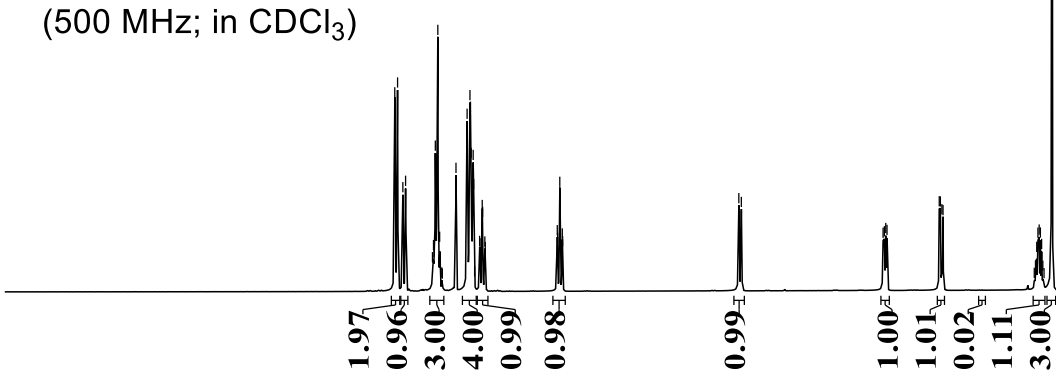

$\begin{array}{lllllllllll}9.5 & 9.0 & 8.5 & 8.0 & 7.5 & 7.0 & 6.5 & 6.0 & 5.5 & \begin{array}{l}5.0 \\ \text { f1 }(\mathrm{ppm})\end{array}\end{array}$

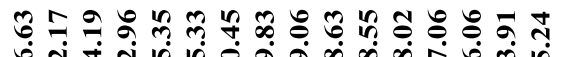

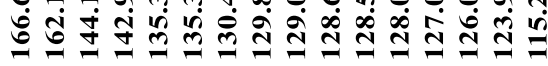

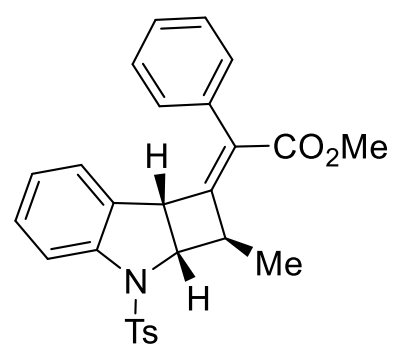

(E)-3ya

$\left(125 \mathrm{MHz}\right.$; in $\mathrm{CDCl}_{3}$ )

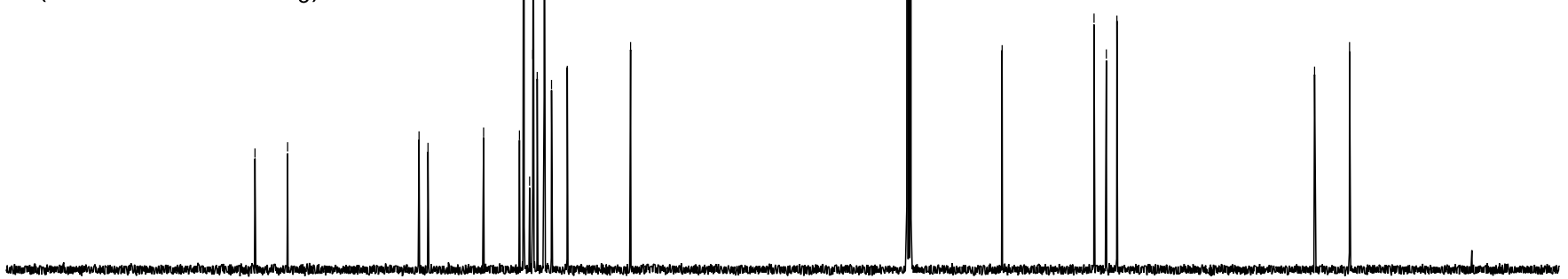

00

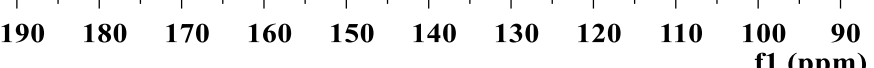

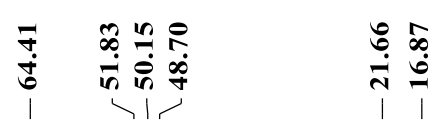


2. X-ray structures of $(E)-3 a a,(Z)-3 a n,(E)-3 a n,(E)-3 a z$ and $(E)-3 z a$

All of the suitable crystals were obtained by interlayer diffusion of hexanes into EtOAc solution at ambient temperature. A colorless crystal of the corresponding compound was mounted on a glass fiber at a random orientation. The data were collected at $100 \mathrm{~K}$ by a diffractometer Rigaku Oxford Diffraction Supernova Dual Source, $\mathrm{Cu}$ at Zero equipped with an Atlas $2 \mathrm{CCD}$ using $\mathrm{Cu} \mathrm{K} \alpha$ radiation (1.54178 $\AA$ ) by using a w scan mode.
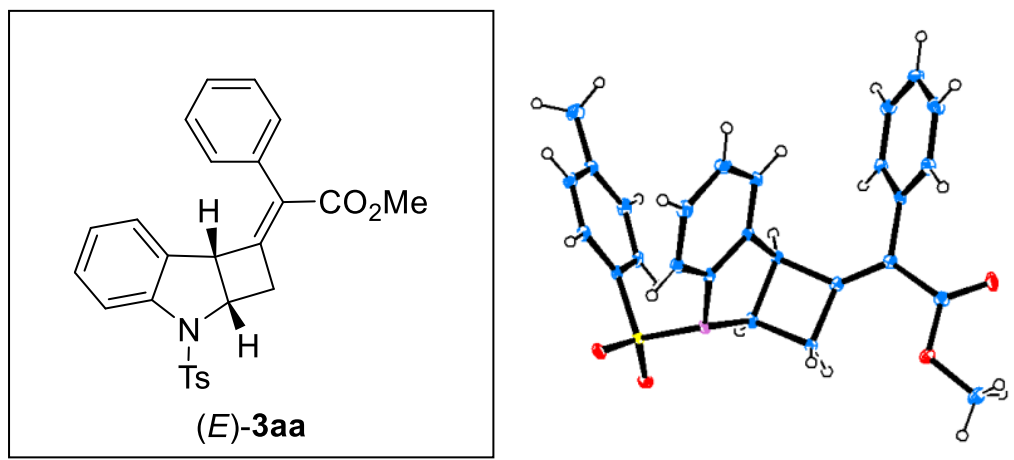

Figure S1 The ORTEP Drawing of (E)-3aa (The ellipsoid contour 30\% probability levels).

Table S1 Crystal data and structure refinement for $(E)-3 \mathbf{a a}$

Identification code

CCDC

Empirical formula

Formula weight

Temperature/K

Crystal system

Space group

$\mathrm{a} / \AA$

b/

c/ ̊

$\alpha /{ }^{\circ}$

$\beta /{ }^{\circ}$

$\gamma /{ }^{\circ}$

Volume $/ \AA^{3}$

Z

$\rho_{\text {calc }} \mathrm{g} / \mathrm{cm}^{3}$

$\mu / \mathrm{mm}^{-1}$
HM592

2047988

$\mathrm{C}_{26} \mathrm{H}_{23} \mathrm{NO}_{4} \mathrm{~S}$

445.51

100.01(10)

orthorhombic

$\mathrm{P} 2{ }_{1}{ }_{12}{ }_{1}$

9.9067(5)

10.0177(5)

22.7535(11)

90

90

90

2258.12(19)

4

1.310

0.176 
$\mathrm{F}(000)$ 936.0

Crystal size $/ \mathrm{mm}^{3}$

$0.12 \times 0.11 \times 0.09$

Radiation $\operatorname{MoK} \alpha(\lambda=0.71073)$

$2 \Theta$ range for data collection $/{ }^{\circ} 4.484$ to 49.984

Index ranges $-9 \leq \mathrm{h} \leq 11,-9 \leq \mathrm{k} \leq 11,-27 \leq 1 \leq 27$

Reflections collected 8202

Independent reflections $3924\left[\mathrm{R}_{\text {int }}=0.0285, \mathrm{R}_{\mathrm{sigma}}=0.0445\right]$

Data/restraints/parameters

$3924 / 0 / 291$

Goodness-of-fit on $\mathrm{F}^{2}$ 1.085

Final $R$ indexes $[\mathrm{I}>=2 \sigma(\mathrm{I})] \quad \mathrm{R}_{1}=0.0366, \mathrm{wR}_{2}=0.0766$

Final $R$ indexes [all data] $\quad R_{1}=0.0399, w_{2}=0.0787$

Largest diff. peak/hole / e $\AA^{-3} 0.17 /-0.38$

Flack parameter $0.44(5)$
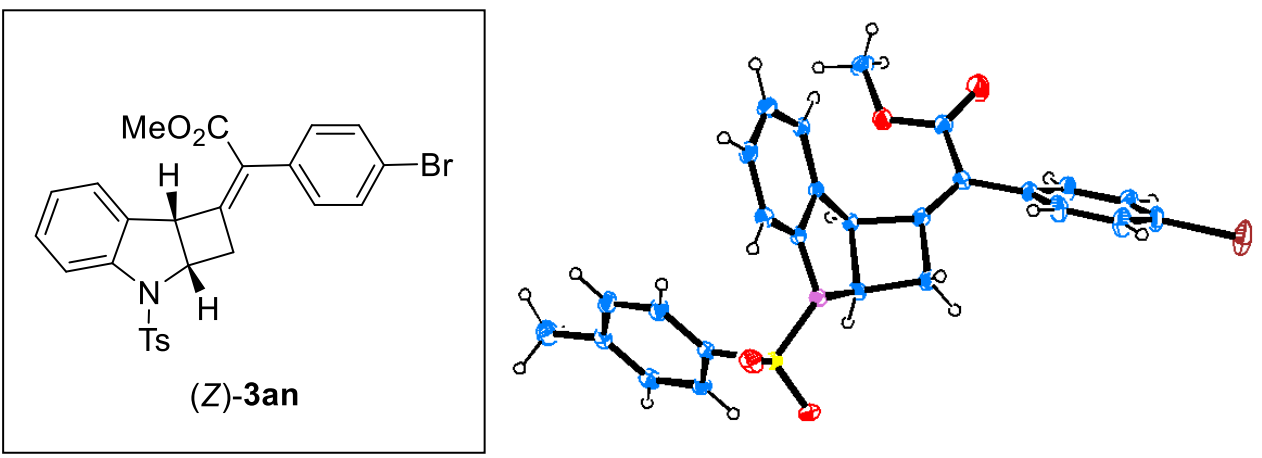

Figure S2 The ORTEP Drawing of (Z)-3an (The ellipsoid contour 30\% probability levels).

Table S2 Crystal data and structure refinement for (Z)-3an

Identification code

CCDC

Empirical formula

Formula weight

Temperature/K

Crystal system

Space group

$\mathrm{a} / \AA$

$\mathrm{b} / \AA$

c/A 3281_0m

2048197

$\mathrm{C}_{26} \mathrm{H}_{22} \mathrm{BrNO}_{4} \mathrm{~S}$

524.41

173

monoclinic

$\mathrm{C} 2 / \mathrm{c}$

42.9622(19)

8.9707(4)

12.1720(7) 
$\alpha /^{\circ}$

$\beta /^{\circ}$

$\gamma /^{\circ}$

Volume $/ \AA^{3}$

Z

$\rho_{\text {calc }} g / \mathrm{cm}^{3}$

$\mu / \mathrm{mm}^{-1}$

$\mathrm{F}(000)$

Crystal size $/ \mathrm{mm}^{3}$

Radiation
90

92.643(2)

90

4686.1(4)

8

1.487

1.877

2144.0

$0.15 \times 0.12 \times 0.07$

$\operatorname{MoK} \alpha(\lambda=0.71073)$

$2 \Theta$ range for data collection $/{ }^{\circ} 4.638$ to 52.754

Index ranges

$-51 \leq \mathrm{h} \leq 53,-11 \leq \mathrm{k} \leq 11,-12 \leq 1 \leq 15$

Reflections collected

17559

Independent reflections

$4754\left[\mathrm{R}_{\mathrm{int}}=0.0566, \mathrm{R}_{\mathrm{sigma}}=0.0550\right]$

Data/restraints/parameters

$4754 / 0 / 300$

Goodness-of-fit on $\mathrm{F}^{2}$

1.024

Final $R$ indexes $[\mathrm{I}>=2 \sigma(\mathrm{I})] \quad \mathrm{R}_{1}=0.0464, \mathrm{wR}_{2}=0.0936$

Final $\mathrm{R}$ indexes [all data] $\quad \mathrm{R}_{1}=0.0788, \mathrm{wR}_{2}=0.1083$

Largest diff. peak/hole / e $\AA^{-3} 1.01 /-1.07$
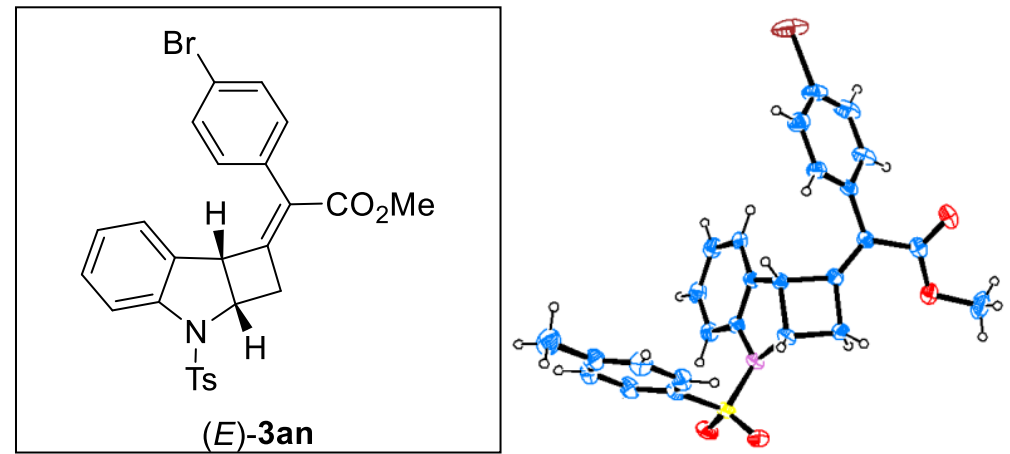

Figure S3 The ORTEP Drawing of (E)-3an (The ellipsoid contour 30\% probability levels). 
SUPPORTING INFORMATION

Table S3 Crystal data and structure refinement for $(E)$-3an

Identification code 3282_0m

$\begin{array}{ll}\text { CCDC } & 2048198\end{array}$

Empirical formula $\quad \mathrm{C}_{26} \mathrm{H}_{22} \mathrm{BrNO}_{4} \mathrm{~S}$

Formula weight $\quad 524.41$

Temperature/K 250

Crystal system monoclinic

Space group $\quad \mathrm{P} 2{ }_{1} / \mathrm{c}$

$\mathrm{a} / \AA \quad 10.8231(6)$

$\mathrm{b} / \AA$

c/Å 19.3267(10)

$\begin{array}{ll}\alpha{ }^{\circ} & 90\end{array}$

$\beta /{ }^{\circ} \quad 102.082(2)$

$\gamma /{ }^{\circ} \quad 90$

Volume $/ \AA^{3} \quad 2381.3(2)$

Z 4

$\rho_{\text {calc }} / \mathrm{cm}^{3} \quad 1.463$

$\mu / \mathrm{mm}^{-1} \quad 1.847$

$\begin{array}{ll}\mathrm{F}(000) & 1072.0\end{array}$

Crystal size $/ \mathrm{mm}^{3} \quad 0.28 \times 0.16 \times 0.11$

Radiation $\quad \operatorname{MoK} \alpha(\lambda=0.71073)$

$2 \Theta$ range for data collection/ $/{ }^{\circ} 5.144$ to 55.072

Index ranges $\quad-12 \leq \mathrm{h} \leq 14,-12 \leq \mathrm{k} \leq 15,-25 \leq 1 \leq 25$

Reflections collected $\quad 24397$

Independent reflections $\quad 5432\left[\mathrm{R}_{\text {int }}=0.0727, \mathrm{R}_{\text {sigma }}=0.0681\right]$

Data/restraints/parameters $\quad 5432 / 0 / 300$

Goodness-of-fit on $\mathrm{F}^{2} \quad 1.024$

Final $\mathrm{R}$ indexes $[\mathrm{I}>=2 \sigma(\mathrm{I})] \quad \mathrm{R}_{1}=0.0522, \mathrm{wR}_{2}=0.1041$

Final $\mathrm{R}$ indexes [all data] $\quad \mathrm{R}_{1}=0.1154, \mathrm{wR}_{2}=0.1289$

Largest diff. peak/hole / e $\AA^{-3} 0.46 /-0.93$ 

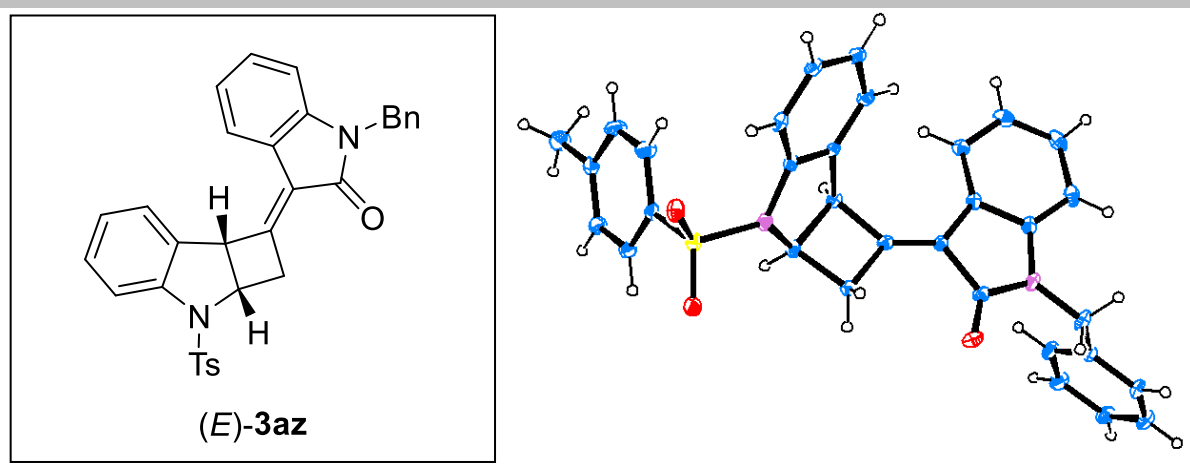

Figure S4 The ORTEP Drawing of (E)-3az (The ellipsoid contour 30\% probability levels).

Table S4 Crystal data and structure refinement for $(E)-\mathbf{3 a z}$

Identification code

CCDC

Empirical formula

Formula weight

Temperature/K

Crystal system

Space group

$\mathrm{a} / \AA$

$\mathrm{b} / \AA$

$\mathrm{c} / \AA$

$\alpha^{\circ}$

$\beta /{ }^{\circ}$

$\gamma /{ }^{\circ}$

Volume $/ \AA^{3}$

$\mathrm{Z}$

$\rho_{\text {calc }} \mathrm{g} / \mathrm{cm}^{3}$

$\mu / \mathrm{mm}^{-1}$

$\mathrm{F}(000)$

Crystal size/ $/ \mathrm{mm}^{3}$

Radiation
$382-2$

2048195

$\mathrm{C}_{32} \mathrm{H}_{26} \mathrm{~N}_{2} \mathrm{O}_{3} \mathrm{~S}$

518.61

$100.0(3)$

triclinic

$\mathrm{P}-1$

9.5394(5)

9.9844(6)

14.3067(8)

96.580(5)

91.240(5)

$111.940(6)$

1252.55(13)

2

1.375

0.168

544.0

$0.13 \times 0.12 \times 0.11$

$\operatorname{Mo~} \operatorname{K} \alpha(\lambda=0.71073)$

$2 \Theta$ range for data collection $/{ }^{\circ} 4.438$ to 50

Index ranges

$-10 \leq \mathrm{h} \leq 11,-9 \leq \mathrm{k} \leq 11,-16 \leq 1 \leq 16$

Reflections collected

8129 
Independent reflections

Data/restraints/parameters

Goodness-of-fit on $\mathrm{F}^{2}$

Final $\mathrm{R}$ indexes $[\mathrm{I}>=2 \sigma(\mathrm{I})] \quad \mathrm{R}_{1}=0.0530, \mathrm{wR}_{2}=0.1301$

Final $R$ indexes [all data] $\quad R_{1}=0.0635, w_{2}=0.1425$

Largest diff. peak/hole / e $\AA^{-3} 0.24 /-0.47$
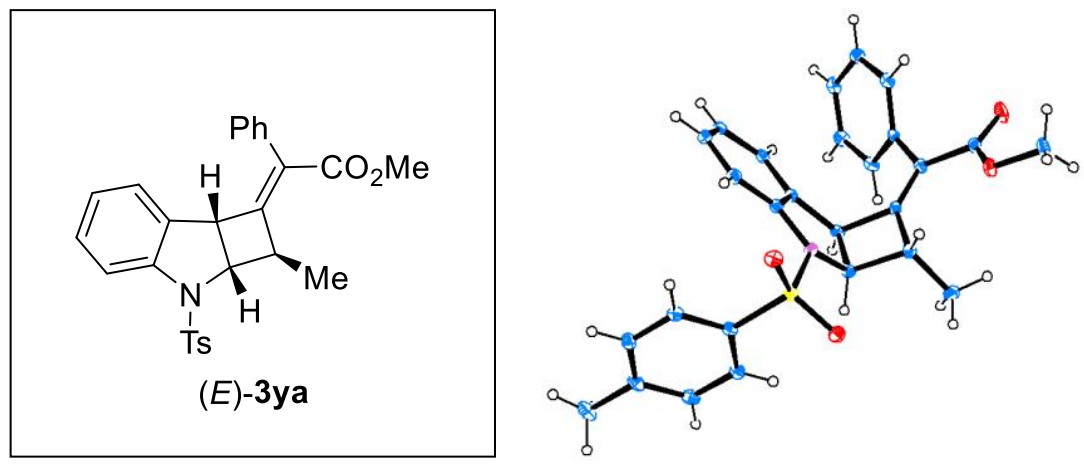

Figure S5 The ORTEP Drawing of (E)-3ya (The ellipsoid contour 30\% probability levels).

Table S5 Crystal data and structure refinement for $(E)-\mathbf{3 y a}$

Identification code

P671_2_2

CCDC

2048196

Empirical formula

$\mathrm{C}_{27} \mathrm{H}_{25} \mathrm{NO}_{4} \mathrm{~S}$

Formula weight

459.54

Temperature/K

99.99(10)

Crystal system

orthorhombic

Space group

Pbca

$\mathrm{a} / \AA$

9.1205(4)

$\mathrm{b} / \AA$

11.5145(5)

c/

44.0271(18)

$\alpha /{ }^{\circ}$

90

$\beta /{ }^{\circ}$

90

$\gamma /{ }^{\circ}$

90

Volume $/ \AA^{3}$

4623.6(3)

Z

$\rho_{\text {calc }} \mathrm{g} / \mathrm{cm}^{3}$ 
$\mu / \mathrm{mm}^{-1} \quad 0.174$

$\mathrm{F}(000) \quad 1936.0$

Crystal size $/ \mathrm{mm}^{3} \quad 0.13 \times 0.12 \times 0.11$

Radiation $\quad \operatorname{Mo~} \operatorname{K\alpha }(\lambda=0.71073)$

$2 \Theta$ range for data collection/ $/{ }^{\circ} 4.834$ to 49.982

Index ranges $\quad-8 \leq \mathrm{h} \leq 10,-13 \leq \mathrm{k} \leq 11,-52 \leq 1 \leq 52$

Reflections collected $\quad 15125$

Independent reflections $\quad 4069\left[\mathrm{R}_{\text {int }}=0.0630, \mathrm{R}_{\text {sigma }}=0.0597\right]$

Data/restraints/parameters 4069/0/301

Goodness-of-fit on $\mathrm{F}^{2} \quad 1.067$

Final $\mathrm{R}$ indexes $[\mathrm{I}>=2 \sigma(\mathrm{I})] \quad \mathrm{R}_{1}=0.0543, \mathrm{wR}_{2}=0.1161$

Final $\mathrm{R}$ indexes [all data] $\quad \mathrm{R}_{1}=0.0742, \mathrm{wR}_{2}=0.1271$

Largest diff. peak/hole / e $\AA^{-3} 0.29 /-0.50$ 